Universidade de São Paulo

Instituto de Astronomia, Geofísica e Ciências Atmosféricas

Departamento de Astronomia

Rodrigo Georgetti Vieira

\title{
Envoltórios circunstelares de estrelas jovens de massa intermediária
}

São Paulo 



\author{
Rodrigo Georgetti Vieira
}

\title{
Envoltórios circunstelares de estrelas jovens de massa intermediária
}

\author{
Tese apresentada ao Departamento de Astrono- \\ mia do Instituto de Astronomia, Geofísica e \\ Ciências Atmosféricas da Universidade de São \\ Paulo como requisito parcial para a obtenção \\ do título de Doutor em Ciências. \\ Área de Concentração: Astronomia \\ Orientadora: \\ Prof.( $\left(^{a}\right)$ Dr. $\left(^{a}\right)$ Jane Cristina Gregorio-Hetem \\ VERSÃO CORRIGIDA \\ (versão original disponível na Unidade)
}

São Paulo 

Às estrelas, que brilham mesmo sem saber porquê. 



\section{Agradecimentos}

Agradeço primeiramente à minha família, pelo apoio incondicional ao caminho que escolhi. Agradeço à Jane, pela orientação e por toda atenção ao longo destes anos. Espero que possamos sempre trabalhar juntos! Agradeço também ao Annibal, que acabou sendo o segundo orientador deste trabalho. Agradeço ao Vincent, por ter me recebido e orientado na França.

Agradeço aos amigos do IAG, pelos cafés e risadas. Ao Alberto, Andressa Ferreira, Andressa Jendreieck, Fellipy, Grazi, Paulinho, Pedro, Phillip e Ti. Ao Ti, agradeço também pelas valiosas discussões à respeito da deconvolução de imagens, que me ajudaram bastante.

Agradeço à turma que ingressou comigo (ou um pouquinho antes) no IAG, pelo caminho compartilhado: Avellar, Felipe, Márcia, Oscaaaaar, Pamela, Raí, Vinicius e Walter.

Agradeço também aos amigos do Sputnik, que conheci pelo interesse em comum: as estrelas! Especialmente ao Osvaldo, Gabriel, Jeferson e Diana. Ao Osvaldo também pelos bate-papos, pelos jogos do "Curíntias" e pelos grandes exemplos.

Agradeço ao pessoal do Coralusp, pela música e pelas amizades. Em especial, ao Alberto e ao naipe dos baixos.

Agradeço aos amigos de sempre: ao Adão, Diogo, Márcio (tiozinho) e Monique. Agradeço muito à Monique, pela amizade sem fronteiras.

Agradeço aos amigos que fiz na Maison do Brasil: Gysa, João Alexandre, Mazinha, Solveig e Yara. Todas essas pessoas transformaram uma época muito difícil em um séjour muito feliz.

Agradeço ao Rama, por todos os conselhos e por todo apoio. Agradeço também pela oportunidade de participar das atividades do Observatório de Valinhos. Levar a Astronomia para além dos muros da Universidade deu um maior significado para o meu trabalho. 
Agradeço muito ao Departamento de Astronomia do IAG, e todas as pessoas que o compõe. Agradeço às secretárias: Cida, Concê, Marina e Regina. Agradeço também aos técnicos: Luis, Marco, Patrícia e Ulisses. Ao longo de todos estes anos, o IAG se tornou sem dúvidas o meu segundo lar.

Agradeço ao Alex, por me acolher para o trabalho de pós-doc, e assim possibilitar que eu continue fazendo o que realmente gosto.

Agradeço à Loren, por todo carinho, paciência e companhia.

Agradeço finalmente à FAPESP, pelo apoio financeiro sob o projeto $n^{o}$ : 2008/01533-4, e à CAPES, pelo apoio financeiro sob o projeto $n^{o}$ : BEX2919/08-7. Todo este trabalho não poderia ser desenvolvido sem o financiamento oferecido por estas agências. 
“There's Venus. There's Mars. And there's Jupiter. And I'm stuck here." Calvin (Bill Watterson) 



\section{Resumo}

As estrelas Herbig Ae/Be (HAeBe) representam os objetos de massa intermediária $\left(2-10 \mathrm{M}_{\odot}\right)$ na pré-sequência principal. Algumas de suas propriedades físicas são pouco compreendidas até o momento. Somente o estudo conjunto das informações fornecidas em diversos comprimentos de onda pode revelar as características do material circunstelar destes objetos. O objetivo deste trabalho de doutorado é analisar sob vários aspectos a estrutura, a composição e a evolução destes ambientes circunstelares. Para realização deste estudo, adotamos a amostra de candidatas a estrelas HAeBe detectadas pelo Pico dos Dias Survey (Vieira et al., 2003). Evitamos as possíveis contaminações desta amostra por estrelas em estágios mais avançados utilizando diagramas de cores, estimativas de extinção e características espectrais. A química da poeira circunstelar foi analisada a partir das propriedades dos espectros ISO disponíveis para nossos objetos. O perfil espectral do silicato em torno de $10 \mu \mathrm{m}$ revelou características evolutivas do material circunstelar. O status evolutivo dos objetos mais embebidos foi determinado por meio de estimativas da massa de seus envoltórios circunstelares. Este estudo indicou que a maior parte desta subamostra se encontra no estágio intermediário entre a Classe $0\left(M_{\text {env }}>>M_{*}\right)$ e a Classe I $\left(\mathrm{M}_{\mathrm{env}}<\mathrm{M}_{*}\right)$ de estrelas jovens. Detalhes da morfologia do disco de PDS340 foram analisados por imagens no infravermelho-médio, obtidas em bancos de dados. Estas observações impuseram vínculos à extensão e orientação espacial do disco nesta faixa espectral. As observações disponíveis em vários comprimentos de onda revelaram características da estrutura e evolução do material circunstelar associado a estrelas HAeBe. A perspectiva do desenvolvimento de um modelo completo que abranja todas estas informações é descrita na conclusão do trabalho. 



\section{Abstract}

Herbig Ae/Be $(\mathrm{HAeBe})$ objects are intermediate mass $\left(2-10 \mathrm{M}_{\odot}\right)$ stars in the premain sequence. Some of their properties remain not well understood to date. Only a full multi-wavelength study is able to reveal a reasonable scenario for their circumstellar material. The purpose of the present work is to study the structure, composition and evolution of these circumstellar enviroments. To address this issues, the sample of HAeBe candidates detected by the Pico dos Dias Survey (Vieira et al., 2003) was adopted. To avoid the contamination by more evolved stars, we developed an analysis based on twocolor diagrams, extinction values and spectral features. The chemistry of the circumstellar dust was studied based on Infrared Space Observatory spectra available to our sample. The silicate feature around $10 \mu \mathrm{m}$ revealed evolutionary information of the circumstellar material. The evolutionary stage of the more embeded sources was determined by estimates of their envelope masses. This study indicates almost all of this sub-sample to be in the intermediate phase between Class $0\left(\mathrm{M}_{\mathrm{env}}>>\mathrm{M}_{*}\right)$ and Class I $\left(\mathrm{M}_{\mathrm{env}}<\mathrm{M}_{*}\right)$. Midinfrared images, retrieved from archive data, introduced morphological constraints to the orientation and extension of the disk associated to PDS340. The available observations for several wavelengths revealed some characteristics of the structure and evolution of the circumstellar material associated to HAeBe stars. The perspective of the development of a complete model, which emcompasses all the available data, is described in the conclusion of this work. 



\section{Lista de Figuras}

1.1 Modelo de disco apresentado por Meeus et al. (2001). . . . . . . . . . . . . 22

1.2 Representantes dos grupos de SED definidos por Sartori et al. (2010)] . . 25

2.1 Espectros ópticos de objetos PDS. . . . . . . . . . . . . . . . . . . 31

2.2 Largura equivalente de $\mathrm{H}_{\alpha}$ versus medidas de extinção. . . . . . . . . . . . 33

2.3 Diagrama de cores AKARI da sub-amostra PDS. . . . . . . . . . . . . . . 35

2.4 Diagrama de cores no IR próximo de sub-amotra PDS. . . . . . . . . . 38

2.5 Diagrama de cores no IR próximo e médio de sub-amostra PDS. . . . . . . 39

3.1 Minerais encontrados em discos protoplanetários . . . . . . . . . . . . . . 42

3.2 Evolucão do perfil de silicato . . . . . . . . . . . . . . . . . . . . . 43

3.3 Espectros ISO disponíveis para obietos HAeBe do PDS. . . . . . . . . . . 46

3.4 Espectros Keck e ISO de sub-amostra de KS05. . . . . . . . . . . . . . . . 48

3.5 Comparacão entre medidas Keck e ISO . . . . . . . . . . . . . . . . . 50

3.6 Espectros ISO/LRS dos objetos de nossa amostra . . . . . . . . . . . . . 52

3.7 Relacão entre o índice espectral IR e a intensidade do silicato. . . . . . . . . 53

3.8 FWHM versus a posicão de máximo (ou mínimo) do silicato. . . . . . . . . 55

3.9 Intensidade em funcão da posicão de pico de emissão . . . . . . . . . . . . . 56

3.10 Convergência do método de GA. . . . . . . . . . . . . . . . . . . 59

3.11 Modelo produzido pelo código DUSTY, sobreposto ao espectro de PDS061. 60

4.1 Evolucão de um objeto estelar jovem. . . . . . . . . . . . . . . . 65

4.2 Diagrama $L_{h o l} \times M_{e n \text {, }}$ para amostra de Classe 0 e Classe I. . . . . . . . . . 66

4.3 Mapa de velocidades em $\mathrm{CH}_{3} \mathrm{CN}$ obtido por Beltrán et al. (2004). . . . . . 71 


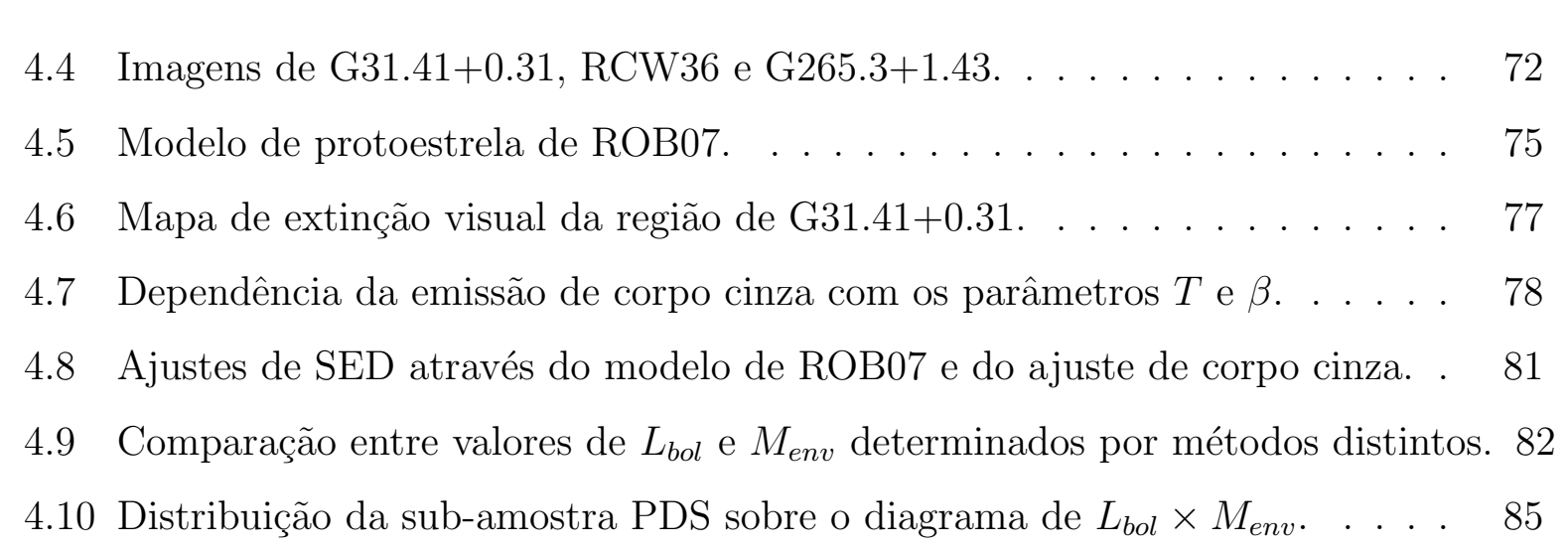

5.1 Transmissão atmosférica calculada pelo código ATRAN (Lord, 1992). . . . 88

5.2 Resolucão espacial de instrumentos astronômicos que operam no IR. . . . . 88

5.3 Comparação entre sensibilidade de algumas missões no IR. . . . . . . . . . 89

5.4 Estrutura de disco de PDS340 proposta por Benisty et al. (2011). . . . . . 91

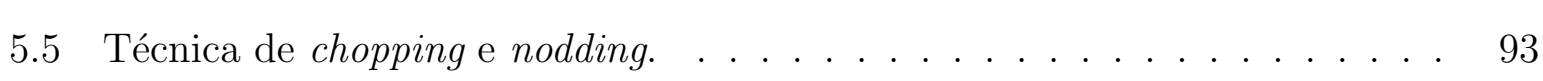

5.6 Modos perpendicular e paralelo de chopping/nodding. . . . . . . . . . . . 93

5.7 Sequência cronológica de uma observacão VISIR. . . . . . . . . . . . . . . 94

5.8 Artefatos produzidos pelo efeito Hammer . . . . . . . . . . . . . . . 96

5.9 Exemplo de correcão de artefatos de imagem VISIR. . . . . . . . . . . . 97

5.10 Comparacão entre diferentes métodos de combinação de imagens VISIR. . 98

5.11 PDS340 na bandas VISIR. . . . . . . . . . . . . . . . 100

5.12 Fluxos calculados confrontados com outras observaç̃es. . . . . . . . . . . 101

5.13 Emissão circunstelar de PDS340 nas bandas VISIR. . . . . . . . . . . . . 103

5.14 Emissão circunstelar de PDS340 nas bandas VISIR - continuação. . . . . . 104

5.15 Imagens deconvoluídas. . . . . . . . . . . . . . . . . . . 106

5.16 Resíduo deconvoluído nas bandas VISIR . . . . . . . . . . . . . . . . 108

5.17 Resíduo deconvoluído nas bandas VISIR - continuação. . . . . . . . . . . . 109

5.18 Ajuste da PSF pela função de Moffat. . . . . . . . . . . . . . . . . . . . . . 109

6.1 SED observada para o obieto PDS340. . . . . . . . . . . . . 115

6.2 Dez melhores ajustes de SED para PDS340, com modelo de ROB07. . . . . 116

6.3 Ajuste de SED para PDS340 com modelo de Dullemond et al. (2001). . . . 118

6.4 Ajuste da SED de PDS340 produzido pelo código RADMC-3D. . . . . . . 121

6.5 Imagens observadas e sintéticas de PDS340 . . . . . . . . . . . . . . 122 


\section{Lista de Tabelas}

2.1 Medidas da $\mathrm{W}_{\mathrm{H}_{\alpha}}, f_{\mathcal{S}_{\boldsymbol{1}}}, E(B-V), A_{\mathrm{V}}$ e $f_{\Delta_{\nu}}$ da sub-amostra PDS. . . . . 32

2.2 Fluxos AKARI e cor $J-[18]$ de sub-amostra PDS . . . . . . . . . . 36

3.1 Objetos HAeBe do PDS com espectro ISO SWS ou IRAS LRS. . . . . . 45

3.2 Medidas do perfil de silicato dos objetos de nossa amostra. . . . . . . . . . 49

3.3 Medidas do perfil de silicato dos objetos de nossa amostra. . . . . . . . . . 51

3.4 Parâmetros do melhor ajuste do espectro de PDS061. . . . . . . . . . . . . 61

4.1 Dados Planck para sub-amostra de HAeBe do PDS. . . . . . . . . . . . . . 69

4.2 Fluxos disponíveis para RCW36\#1. RCW36\#2 e G265.3+1.43. . . . . . 73

4.3 Resultados do ajuste de SED com o modelo de ROB07. . . . . . . . . . . 79

4.4 Parâmetros determinados pelo ajuste de corpo cinza. . . . . . . . . . . . . 80

5.1 Dados das estrelas padrões utilizadas $\ldots \ldots \ldots$. . . . . . . . . . . . . . 99

5.2 Parâmetros morfólogicos de PDS340 . . . . . . . . . . . . . . . . . 110

6.1 Parâmetros determinados pelo ajuste do modelo de ROB07. . . . . . . . 117 



\section{Sumário}

1. Introducãd . . . . . . . . . . . . . . . . . . . . . . . . . . . . . . . . . . . 19

1.1 Estrelas Herbig Ae/Be do PDS . . . . . . . . . . . . . . . . . . 20

1.2 Classificacões segundo o formato da SED . . . . . . . . . . . . . . . 21

1.3 Organização da tese . . . . . . . . . . . . . . . . . . . . . . . . . 26

2. Descontaminacão da amostra . . . . . . . . . . . . . . . . . . . . . . 29

2.1 Características espectrais . . . . . . . . . . . . . . . . . . . . . . 30

2.2 Características circunstelares . . . . . . . . . . . . . . . . . . . . . . . 33

2.3 Diagramas de cores . . . . . . . . . . . . . . . . . . . . . . . . . . 34

2.4 Refinamento da amostra . . . . . . . . . . . . . . . . . . . . . . 40

3. Química da poeira circunstelas f. . . . . . . . . . . . . . . . . . . 41

3.1 Sub-amostra PDS . . . . . . . . . . . . . . . . . . . . . . . . 44

$3.2 \quad$ o perfil do silicato $\ldots \ldots \ldots \ldots$. . . . . . . . . . . . . . 47

3.2 .1 Comparacão: ISO $\times$ Keck $\ldots \ldots \ldots \ldots \ldots \ldots$. . . . . . . 47

3.2 .2 Resultados . . . . . . . . . . . . . . . . . . . 51

3.3 Composição dos grãos de poeira . . . . . . . . . . . . . . . . . . . 57

4. Conexão entre a massa do envoltório e o estágio evolutivo . . . . . . . . . . 63

4.1 Obietos selecionados . . . . . . . . . . . . . . . . . . . . . . . . 68

4.1.1 Sub-amostra PDS com dados Planck . . . . . . . . . . . . . . 68

4.1 .2 Candidatas a protoestrelas massivas . . . . . . . . . . . . . . . . . 69

4.2 Metodologia . . . . . . . . . . . . . . . . . . . . . . 73 
4.2.1 Modelo de Robitaille et al. (2007) . . . . . . . . . . . . . . . . 74

4.2.2 Aproximaç̃o de corpo cinza . . . . . . . . . . . . . . . . 76

4.3 Resultados . . . . . . . . . . . . . . . . . . . . . . . . 78

4.4 Discussão $\ldots \ldots \ldots \ldots$

5. Morfologia no infravermelho médio . . . . . . . . . . . . . . . . . 87

5.1 Observacões VISIR $\ldots \ldots \ldots \ldots$

5.1 .1 Objeto de estudo: PDS340 . . . . . . . . . . . . . . . . . 90

5.1 .2 Descricão do instrumento . . . . . . . . . . . . . . . . . . . 91

5.2 Reducão dos dados . . . . . . . . . . . . . . . . . . . . . . 94

5.2 .1 Descricão das imagens brutas . . . . . . . . . . . . . . . . . . . 94

5.2 .2 Rotinas de redução . . . . . . . . . . . . . . . . . . 95

5.3 Análise das imagens . . . . . . . . . . . . . . . . . . . . . . 99

5.3.1 Subtracão da componente estelar . . . . . . . . . . . . . . . . . . . 101

5.3 .2 Deconvolução com a PSF . . . . . . . . . . . . . . . . . . . 102

5.4 Discussão . . . . . . . . . . . . . . . . . . . . . . 109

6. Conclusões . . . . . . . . . . . . . . . . . . . . . . . 113

6.1 Comparacão entre os modelos de SED . . . . . . . . . . . . . . . . . 115

6.2 Um modelo completo . . . . . . . . . . . . . . . . . . . . . . . . . . . . 119

6.3 Perspectivas . . . . . . . . . . . . . . . . . . . . 123

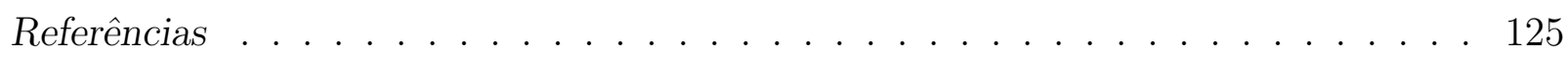

\begin{tabular}{|l|l}
\hline Apêndice & 137
\end{tabular}

A. Lista de acrônimos e convençốes do texto . . . . . . . . . . . . . . . . . . . . . . 139

B. Artigo publicado com resultados parciais obtidos durante o doutorado . . . . . . 143 
Capítulo 1

\section{Introdução}

O estudo do material circunstelar associado a estrelas jovens representa em última instância a compreensão de nossas próprias origens. A descoberta de um número crescente de exoplanetas vem estreitando a conexão entre o colapso do gás e poeira na nuvens primordiais e a formação planetária.

Esta tese tem como objetivo analisar sob vários aspectos a estrutura e a evolução do material ao redor de estrelas de pré-sequência principal (PMS). Este tema é bastante amplo, dado que o cenário de formação e evolução das estrelas depende fortemente do regime de massa envolvido. Por isso, restringimos nosso estudo aos objetos Herbig Ae/Be (HAeBe), que representam a classe de estrelas jovens de massa intermediária $\left(2-10 \mathrm{M}_{\odot}\right)$.

Esta classificação foi primeiramente proposta por Herbig (1960), que sugere neste trabalho a natureza jovem destes objetos. O artigo de revisão de Waters e Waelkens (1998) fornece uma versão atual da definição desta classe de objetos:

- Tipo espectral A ou B, com linhas de emissão;

- Excesso de emissão infravermelha (IR) devido à presença de poeira circunstelar quente e/ou fria;

- Classe de luminosidade de III a V.

O segundo critério tem como propósito excluir as estrelas Be "clássicas", dado que o excesso IR destes objetos é devido à emissão livre-livre de gás ionizado contido no disco 
circunstelar (e.g., Waters e Marlborough 1994). O último item exclui as supergigantes B[e] e estrelas de pós-sequência principal.

\subsection{Estrelas Herbig Ae/Be do PDS}

O Pico dos Dias Survey (PDS - Gregorio-Hetem et al. 1992, Torres et al. 1995, Torres 1999) realizou uma busca por estrelas jovens, selecionadas a partir de critérios de cores IRAS (InfraRed Astronomical Satellite).

Como resultado, foram descobertas cerca de 200 estrelas jovens não catalogadas anteriormente. Vieira et al. (2003) publicaram a lista de 108 candidatas a estrelas HAeBe observadas pelo PDS. Essas estrelas apresentam a linha de $\mathrm{H}_{\alpha}$ em emissão, excesso na emissão IR e indícios de classe espectral recente.

Outro resultado surpreendente obtido pelo PDS foi a descoberta de objetos que não são de natureza jovem. Entre eles, cerca de 20 estrelas gigantes vermelhas ricas em lítio (Gregorio-Hetem et al. 1993, Castilho et al. 2000) e um quasar (Torres et al., 1997). Essas detecções mostram que a seleção de candidatas a partir das cores no IR distante pode revelar condições físicas diferentes para uma mesma característica de emissão de poeira. Esse é o caso de Hen 3-1475, uma das candidatas HAeBe do PDS, identificada como protonebulosa planetária (PPN - Rodrigues et al. 2003).

Cerca de $1 / 3$ das candidatas a HAeBe detectadas pelo PDS apresentam indícios que podem enquadrá-las no mesmo caso de Hen 3-1475. Este fato nos motivou a estudar durante o projeto de mestrado uma amostra de objetos dos mais embebidos, cuja estrutura circunstelar poderia corresponder tanto a um objeto jovem como um evoluído.

No mestrado, estudamos a natureza de uma amostra de 27 objetos selecionada entre as candidatas HAeBe do PDS. Estes alvos apresentam a distribuição espectral de energia (SED) típica de um objeto jovem bastante embebido, mas que também pode se assemelhar a uma estrela mais evoluída, já na fase de transição entre o ramo assintótico das gigantes e as nebulosas planetárias (as pós-AGB).

Buscando separar as estrelas PMS das pós-AGB, foram avaliados os possíveis indicadores do estágio evolutivo destes objetos, tais como as fotometrias na faixa do óptico; IR 
próximo, médio e distante; distribuição espacial e possível associação com complexos de formação estelar e características espectrais disponíveis. Também utilizamos modelos simples para a distribuição do material circunstelar destes objetos, reproduzindo suas SEDs. Sete entre os objetos da amostra apresentaram características condizentes com objetos pós-AGB.

Dado que a seleção dos alvos do PDS foi feita com base nas cores IRAS, ela em princípio não contém nenhum viés a priori acerca da posição espacial ou estágio evolutivo dos objetos. Além disto, a lista de objetos apresentada por Vieira et al. (2003) abrange um grande intervalo de massas e estágios evolutivos das estrelas HAeBe, constituindo uma base bastante completa para nossos propósitos. Por estas razões, decidimos adotar neste trabalho a amostra de objetos HAeBe detectados pelo PDS.

\subsection{Classificações segundo o formato da SED}

A classificação dos objetos HAeBe pode se mostrar útil para revelar características globais desta classe, sugerindo inclusive informações evolutivas. Meeus et al. (2001) realizaram a análise dos espectros ISO (Infrared Space Observatory) de uma amostra de 14 HAeBes consideradas isoladas (i.e., não associadas a regiões de formação estelar). Eles perceberam que a contribuição do contínuo da emissão IR exibida por estes espectros pode ser reproduzida por uma lei de potência que apresenta para alguns casos uma componente extra representada pela emissão de corpo negro (BB). Esta emissão de BB é compatível com uma faixa de temperaturas que varia entre $100 \mathrm{~K}$ e $200 \mathrm{~K}$ aproximadamente. Além disso, as características espectrais referentes aos grãos de algumas espécies mostraram-se presentes em uns e ausentes em outros objetos da amostra. Baseando-se neste comportamento, Meeus et al. (2001) classificaram os objetos de sua amostra nos seguintes grupos:

- Grupo I: objetos cujo contínuo é reproduzido por uma lei de potência mais a contribuição de BB.

- Grupo II: contínuo é bem representado apenas por uma lei de potência.

Além disso, define-se o subgrupo a para representar a presença de características espectrais dos grãos, enquanto $b$ denota sua ausência. 
Meeus et al. (2001) interpretaram as diferentes distribuições de energia como um reflexo direto da estrutura circunstelar associada aos objetos observados. O perfil de emissão dos silicatos deve ser proeminente quando a região do disco mais próxima da estrela se encontra em um regime opticamente fino. Já a componente de emissão de BB deve ser produzida pela regiões mais externas do disco, quando sua superfície é irradiada pela fonte central. A Figura 1.1 exibe a representação esquemática da interpretação geométrica de Meeus et al. (2001). Nesta proposta, a borda interna do disco (Região I) é responsável pelo presença do perfil do silicato. A componente de BB da SED é produzida pela região externa do disco (Região III), quando esta é atingida pela radiação estelar (painel superior). No painel inferior, a parte mais escura da Região I indica um regime opticamente espesso.

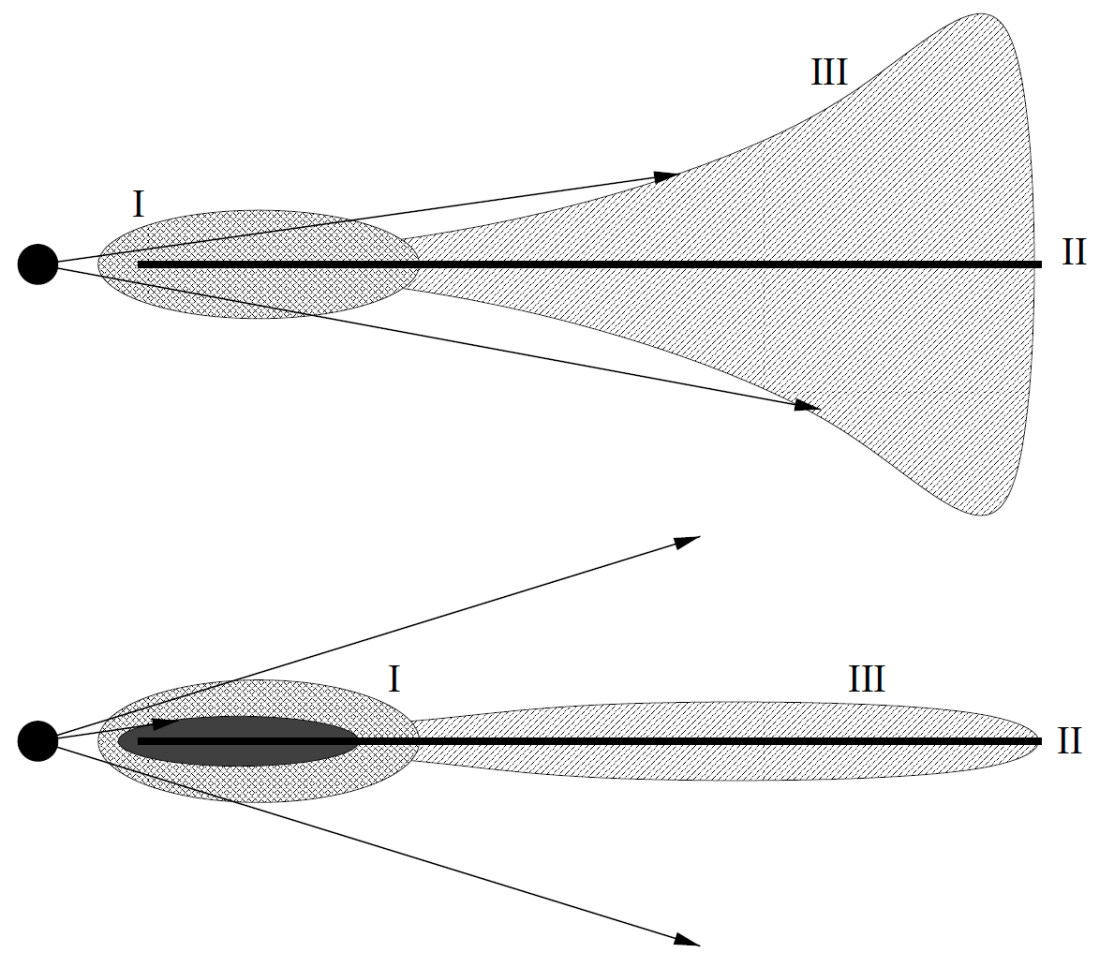

Figura 1.1: Modelo de disco apresentado por Meeus et al. (2001). A Região I representa a componente do disco mais interna $(\lesssim 10 \mathrm{AU})$ e opticamente fina, a Região II uma componente geometricamente fina e opticamente espessa, e a Região III a porção mais externa do disco. Figura adaptada do mesmo trabalho. 
A inclinação da SED entre a região do óptico e do IR distante tem sido usada como critério de classificação de diferentes categorias dos objetos jovens e também das candidatas a pós-AGB. Assim como no caso das estrelas T Tauri, a SED das HAeBe também pode ser um indicativo do status evolutivo dos objetos, supondo que o disco protoestelar se desfaz com o tempo durante a PMS.

Sartori et al. (2003) propuseram um esquema de classificação das HAeBe a partir da análise da SED do objeto. Ele é baseado no valor do índice espectral $\beta$ definido por Torres (1999), expresso por

$$
\beta=0.75 \times \log \left(\mathrm{F}_{12 \mu \mathrm{m}} / \mathrm{F}_{\mathrm{V}}\right)-1 .
$$

A estimativa da fração da contribuição circunstelar foi obtida por meio de um modelo simplificado que propõe uma estrutura composta por objeto central envolvido por um disco fino opticamente espesso e um envoltório esférico de poeira (Gregorio-Hetem e Hetem, 2002). A SED observada é reproduzida considerando-se diferentes leis de temperatura para as componentes individuais do sistema (estrela, disco e envoltório). A partir deste modelo, pode-se calcular a porcentagem da emissão circunstelar com relação à emissão total 1:

$$
f_{S c}=\frac{L_{t o t a l}-L_{*}}{L_{t o t a l}}
$$

sendo $L_{*}$ a contribuição de origem estelar. Desta forma, foram definidos os seguintes grupos:

- Grupo 1: $\beta>1$, indicando que a emissão no IR médio é maior que a emissão no visível. A contribuição circunstelar $f_{S c}$ é maior do que $70 \%$.

\footnotetext{
${ }^{1}$ Na definição original do trabalho de Sartori et al. (2010), este parâmetro é designado por $S_{c}$.
} 
- Grupo 2: $-1 \leq \beta \leq 0$, indicativo de que a emissão no IR-médio é comparável ou menos importante do que a observada no óptico. A fração de sua contribuição circunstelar está em um intervalo entre $10 \%$ e $70 \%$.

- Grupo 3: $\beta<-1$, característico de uma emissão no IR muito menor do que no óptico. Neste caso, $f_{S c}<10 \%$. A Figura 1.2 mostra exemplos de SEDs de representantes destes três grupos.

Esta classificação segue a idéia de um cenário evolutivo das HAeBe, semelhante ao proposto por Malfait et al. (1998). O Grupo 1 corresponde a objetos mais embebidos, como é o caso de $\mathrm{R}$ CrA, enquanto o Grupo 3 representa estrelas com discos de poeira mais evoluídos (e.g., $\beta$ Pictoris e Vega). O Grupo 2 é a fase de transição entre estas duas categorias extremas.

No entanto é importante notar que objetos em diferentes fases de evolução também podem exibir excesso no IR distante devido à emissão circunstelar. É este fato que gera a confusão entre as candidatas a HAeBe do Grupo 1 com objetos do tipo pós-AGB e outros indicativos da real natureza das fontes devem ser levados em consideração. 


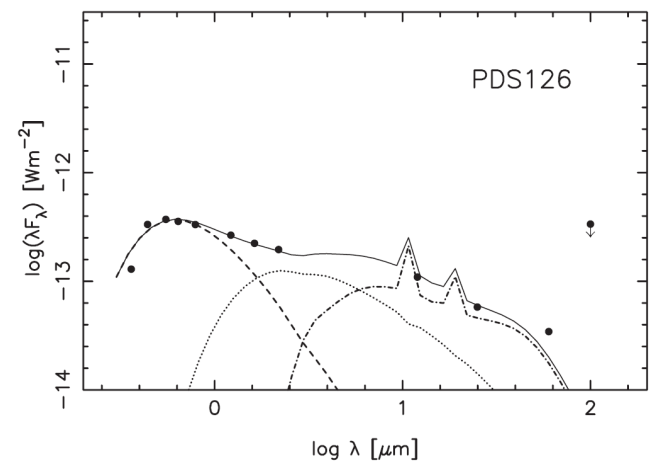

(a) PDS126: exemplo de SED de objeto do Grupo 1.

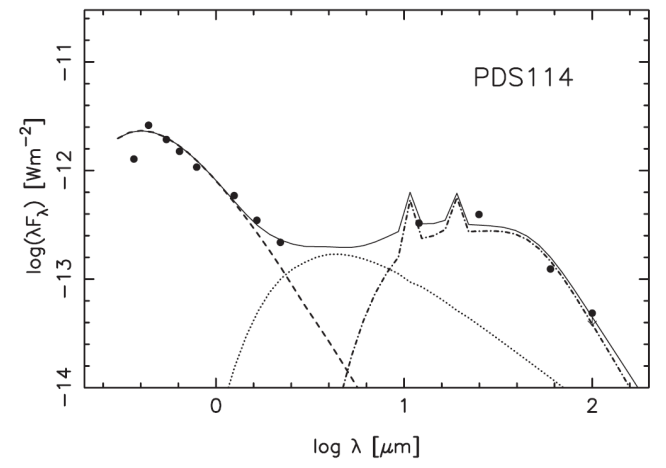

(b) PDS114: exemplo de SED de objeto do Grupo 2.

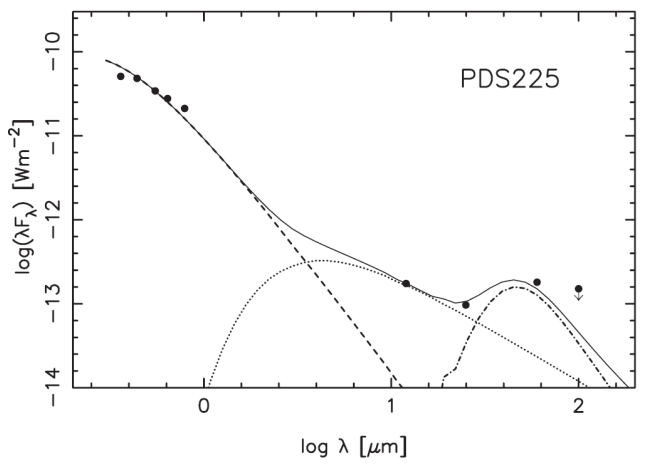

(c) PDS225: exemplo de SED de objeto do

Grupo 3.

Figura 1.2: Representantes dos grupos de SED definidos por Sartori et al. (2010). As curvas teóricas foram calculadas a partir de um modelo de dupla componente circunstelar, descrito por Gregorio-Hetem e Hetem (2002). A linha tracejada representa a emissão da fonte central; a pontilhada indica a contribuição do disco geometricamente fino e opticamente espesso; a linha traço-pontilhada mostra o fluxo produzido pelo envoltório esférico. A linha cheia representa a soma da emissão de todas as componentes. 


\subsection{Organização da tese}

Os capítulos a seguir descrevem o trabalho desenvolvido durante o período de doutorado. Cada um deles contém uma introdução ao seu respectivo assunto, de forma a tornar sua leitura independente 2 dos outros capítulos.

Nosso estudo começa na própria identificação dos objetos jovens, por vezes confundidos com estrelas já na pós-sequência principal associadas a grandes quantidades de poeira circunstelar (Vieira et al., 2011). O Capítulo 2 apresenta a continuidade do trabalho de mestrado, onde aprofundamos o estudo da classificação das estrelas HAeBe mais embebidas do PDS, com o objetivo de refinar a amostra analisada no presente trabalho.

A determinação da química dos grãos pode revelar características da estrutura e da evolução do material circunstelar. As características intrínsecas ao perfil de emissão dos silicatos está intimamente conectada aos parâmetros ambientais que descrevem os discos, e ao seu estágio evolutivo (Kessler-Silacci et al., 2005). O Capítulo 3 explora estas conexões, discutindo a evolução e a composição da química da poeira associada aos objetos de nossa amostra.

Seguindo a proposta de André et al. (2000), adotamos o diagrama de luminosidade bolométrica versus a massa do envoltório de estrelas jovens para analisar o status evolutivo destes objetos. Apresentamos no Capítulo 4 o estudo da distribuição dos objetos PDS sobre este diagrama.

O desenvolvimento atual da instrumentação associada aos grandes telescópios permite o estudo dos detalhes morfológicos do material circunstelar. O Capítulo 5 apresenta a análise desta morfologia, realizado a partir de imagens no IR médio de alta resolução espacial obtidas pelo instrumento VISIR no VLT.

O Capítulo 6 sumariza todos os resultados apresentados, discutindo suas interconexões. Finalmente, descrevemos como perspectiva de continuação deste trabalho o desenvolvi-

\footnotetext{
${ }^{2}$ Embora seus temas sejam interdependentes, conforme discutiremos no Capítulo 6
} 
mento de um modelo que compreenda todas as análises discutidas ao longo desta tese. 
Capítulo 2

\section{Descontaminação da amostra}

A classificação dos objetos de nossa amostra representa o ponto de partida para o estudo do material circunstelar das estrelas HAeBe. Neste capítulo, damos atenção especial aos objetos mais embebidos do Grupo 1, os quais foram inicialmente estudados durante o trabalho de mestrado. Conforme descrito no Capítulo1, há indícios da presença de objetos de pós-sequência principal nesta sub-amostra.

É importante ressaltar que os resultados aqui apresentados representam parte das atividades desenvolvidas durante o trabalho doutorado, consistindo de análises complementares distintas daquelas descritas na dissertação de mestrado. Os resultados dessa parte do trabalho foram publicados no artigo "Distinguishing post-AGB impostors in a sample of pre-main sequence stars" (Vieira et al. 2011 - Apêndice B), que teve como objetivo principal apontar os melhores alvos a serem analisados com maior detalhamento no presente trabalho.

Ao longo do trabalho de mestrado, estudamos vários possíveis indicadores da natureza dos objetos mais embebidos da amostra PDS. Analisamos a existência de associação com regiões formadoras de estrelas, a distribuição dos objetos da amostra em diagramas de cor nas faixas do óptico, infravermelho (IR) próximo, médio e distante, e a compatibilidade da distribuição espectral com uma estrutura circunstelar de uma ou duas componentes.

Em adição ao trabalho de mestrado, foram incluídos critérios baseados em características espectrais detectadas nas observações disponíveis do PDS, além da comparação 
da distribuição da amostra em diagramas de cores com conjuntos de objetos de natureza bem conhecidas. Dados obtidos pelo satélite AKARI (em $9 \mu \mathrm{m}$ e $18 \mu \mathrm{m}$ ) também foram incluídos em nossa análise. As implementações à análise e os resultados deste aprofundamento são descritos nas seções a seguir.

\subsection{Características espectrais}

Os espectros em comprimentos de onda ópticos obtidos pela missão do PDS são mostrados na Figura 2.1. Eles contêm as linhas nebulares [OI] (6302 A, $6365 \AA)$ e [SII] (6718 A, $6732 \AA$ ), entre outras. Os espectros de algumas fontes não são exibidos por razões distintas: eles possuem apenas a emissão em $\mathrm{H}_{\alpha}$ (PDS 174, 198, 290, 394, 520 e 543), contêm muito ruído (PDS168, 207 e 431), o intervalo espectral entre $6300 \AA$ e $6500 \AA$ não foi coberto (PDS 18, 27, 37, 67 e 141), ou o espectro não estava disponível (PDS 257).

Quase metade de nossa amostra exibe a emissão da linha de [OI] $6302 \AA$, mas apenas PDS 371 apresenta características similares àquelas encontradas em objetos evoluídos como nebulosas planetárias (e.g., linhas nebulares de [SII]; Pereira e Miranda 2007). Como a presença desta linha não é comum a toda amostra, ou é incerta, ela não foi usada para o diagnóstico da natureza de nossos objetos.

A largura equivalente da linha de $\mathrm{H}_{\alpha}\left(\mathrm{W}_{\mathrm{H}_{\alpha}}\right)$ dos alvos de nossa amostra foi medida para todos os casos possíveis e se encontra listada na Tabela 2.1, Nos casos de espectros com baixa razão de sinal-ruído, nós consideramos os valores de $\mathrm{W}_{\mathrm{H}_{\alpha}}$ publicados no catálogo do PDS (obtidos a partir dos mesmos espectros).

A maioria dos espectros exibe intensas linhas de $\mathrm{H}_{\alpha}$ em emissão, a menos de dois deles que aparecem em absorção (PDS290 e 394). A variabilidade das linhas, a qual é comum

entre as estrelas na PMS, é uma possível explicação para a presença de linhas relativamente mais fracas em $\mathrm{H}_{\alpha}$. 


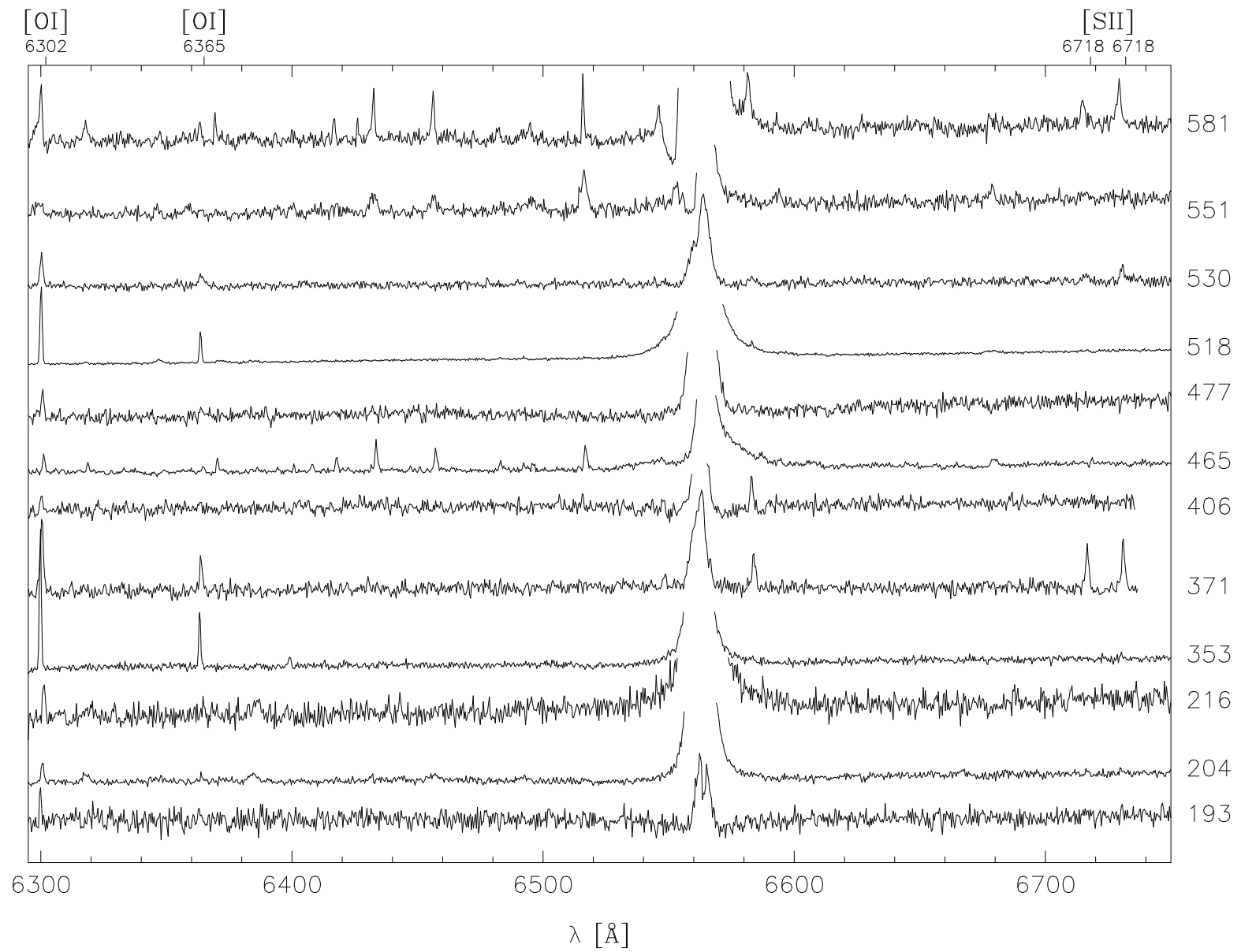

Figura 2.1: Espectros ópticos de parte da amostra, identificados pelo número PDS ao lado direito. $\mathrm{O}$ perfil de $\mathrm{H}_{\alpha}$ não é mostrado por inteiro para ressaltar detalhes de outras linhas de emissão. A escala de intensidade é arbitrária. 
Tabela 2.1 - Lista de objetos mais embebidos do Grupo 1 das candidatas HAeBe detectadas pelo PDS, apresentando os valores estimados da largura equivalente de $\mathrm{H}_{\alpha}\left(\mathrm{W}_{\mathrm{H} \alpha}\right) ; f_{S c}$ a fração da luminosidade circunstelar frente à total, $E(B-V)$ o excesso de cor calculado, $A_{V}$ a extinção visual calculada por Dobashi et al. (2005) e $f_{A v}$, uma medida da fração do avermelhamento circunstelar frente ao total (definida na Seção 2.2).

\begin{tabular}{|c|c|c|c|c|c|c|}
\hline PDS & 2MASS & $\begin{array}{c}\mathrm{W}_{\mathrm{H} \alpha} \\
\AA\end{array}$ & $f_{S c}$ & $\begin{array}{c}E(B-V) \\
\text { mag }\end{array}$ & $\begin{array}{l}A_{V} \\
\text { mag }\end{array}$ & $f_{A v}$ \\
\hline 018 & 05534254-1024006 & 40 & 0.9 & 1.42 & $1.3 \pm 0.3$ & 0.7 \\
\hline 027 & 07193593-1739180 & 88 & 0.96 & 1.41 & $1.2 \pm 0.2$ & 0.7 \\
\hline 037 & $10100032-5702073$ & 105 & 0.93 & 1.6 & $2.8 \pm 0.4$ & 0.4 \\
\hline 067 & $13524285-6332492$ & 90 & 0.97 & 1.56 & $1.4 \pm 0.3$ & 0.7 \\
\hline 141 & $12531722-7707106$ & 40 & 0.97 & - & $3.8 \pm 0.8$ & 0.0 \\
\hline 168 & $04305028+2300088$ & 8 & 0.75 & 2.14 & $0.9 \pm 0.6$ & 0.0 \\
\hline 174 & 05065551-0321132 & 65 & 0.97 & 1.01 & $1.2 \pm 0.2$ & 0.6 \\
\hline 193 & 05380931-0649166 & 12 & 0.7 & 1.34 & $2.6 \pm 0.6$ & 0.4 \\
\hline 198 & $05385862-0716457$ & 8.5 & 0.85 & 1.13 & $2.6 \pm 0.6$ & 0.3 \\
\hline 204 & $05501389+2352177$ & 245 & 0.95 & 1.1 & $0.9 \pm 0.4$ & 0.7 \\
\hline 207 & $06071539+2957550$ & 5 & 0.89 & 1.2 & $0.3 \pm 0.5$ & 0.9 \\
\hline 216 & $06235631+1430280$ & 200 & 0.92 & 1.19 & $0.8 \pm 0.2$ & 0.8 \\
\hline 257 & 07414105-2000134 & 15 & 0.89 & 0.89 & $0.6 \pm 0.1$ & 0.8 \\
\hline 290 & $09261107-5242269$ & -7 & 0.94 & 0.66 & $1.6 \pm 0.2$ & 0.2 \\
\hline 353 & $12222318-6317167$ & 200 & 0.89 & 0.99 & $1.3 \pm 0.2$ & 0.6 \\
\hline 371 & 13473141-3639495 & 40 & 0.79 & 1.4 & $0.1 \pm 0.1$ & 1.0 \\
\hline 394 & $15351712-6159041$ & -2 & 0.86 & 0.44 & $0.7 \pm 0.2$ & 0.5 \\
\hline 406 & $16050392-3945034$ & 13 & 0.82 & 0.4 & $1.2 \pm 0.5$ & 0.0 \\
\hline 431 & $16545918-4321496$ & 8 & 0.94 & 0.52 & $1.6 \pm 0.4$ & 0.0 \\
\hline 465 & 17451419-1756469 & 110 & 0.92 & 1.23 & $0.6 \pm 0.2$ & 0.8 \\
\hline 477 & $18003031-1647259$ & 120 & 0.87 & 1.37 & $2.6 \pm 0.4$ & 0.4 \\
\hline 518 & $18273952-0349520$ & 600 & 0.91 & 2.3 & $5.4 \pm 1$ & 0.2 \\
\hline 520 & $18300616+0042336$ & 33 & 0.84 & 1.26 & $5.5 \pm 0.8$ & 0.0 \\
\hline 530 & $18413436+0808207$ & 28 & 0.82 & 0.47 & $1 \pm 0.3$ & 0.3 \\
\hline 543 & $18480066+0254170$ & 0.8 & 0.94 & 1.79 & $4.6 \pm 0.6$ & 0.2 \\
\hline 551 & $18552297+0404353$ & 50 & 0.96 & 1.92 & $6.3 \pm 0.7$ & 0.0 \\
\hline 581 & $19361890+2932500$ & 200 & 0.94 & 0.89 & $0 \pm 0.1$ & 1.0 \\
\hline
\end{tabular}




\subsection{Características circunstelares}

A Figura 2.2 mostra a comparação entre a fração de luminosidade circunstelar, dada por $f_{S c}=\left(L_{t o t a l}-L_{*}\right) / L_{t o t a l}$, e a largura equivalente $\mathrm{em}_{\alpha}$. A luminosidade total $L_{\text {total }}$ e a contribuição estelar $L_{*}$ foram estimadas por meio do modelo de SED de Gregorio-Hetem e Hetem (2002). Na figura, podemos notar que a distribuição de objetos com $\mathrm{W}_{\mathrm{H}_{\alpha}} \lesssim 50 \AA$ se estende por todo o domínio de $f_{S c}$. Em contrapartida, objetos que apresentam linhas de emissão intensas estão principalmente concentrados na região definida por $f_{S c}>0.87$.

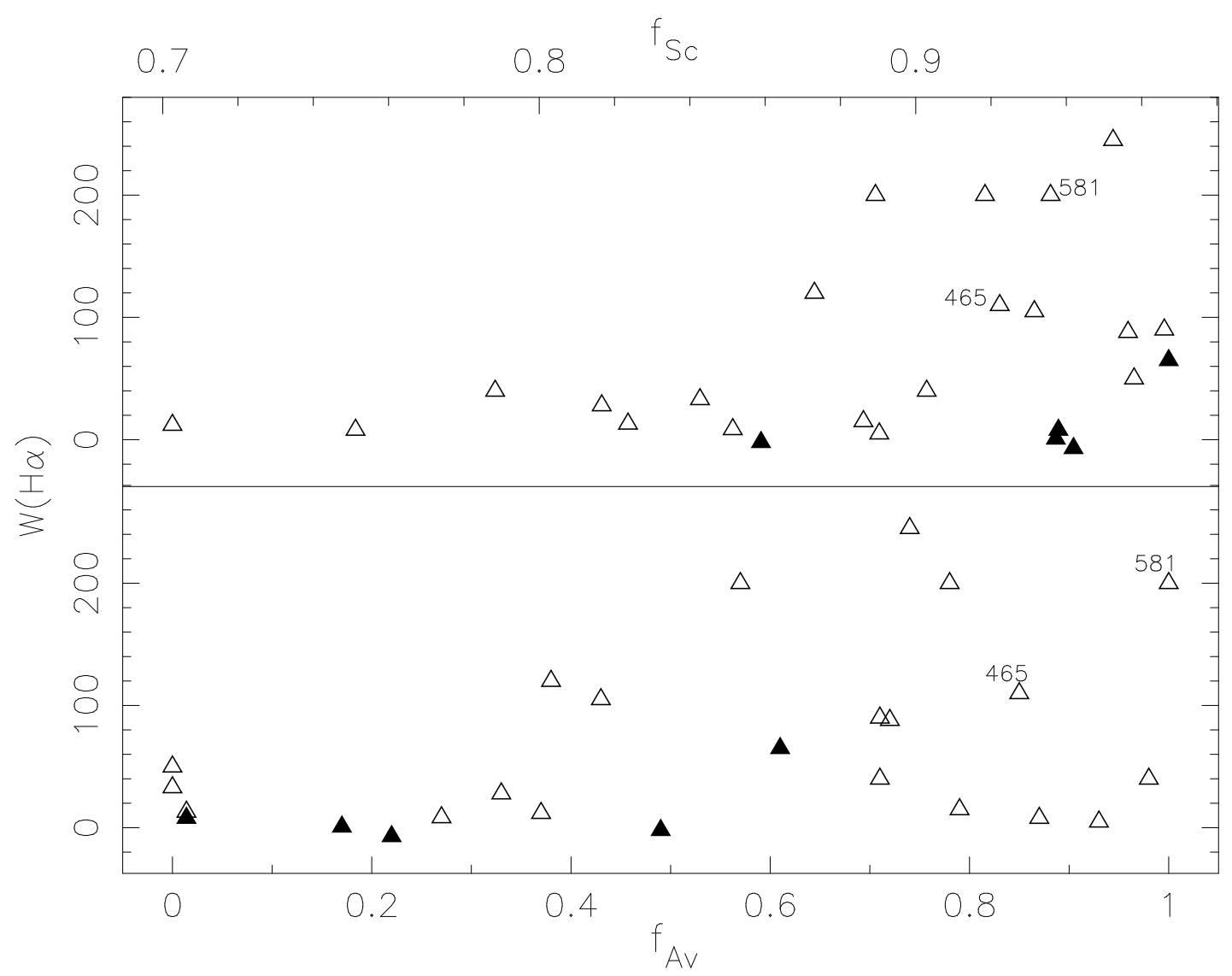

Figura 2.2: $\mathrm{O}$ painel superior exibe a largura equivalente da linha de $\mathrm{H}_{\alpha}$ como função da fração da luminosidade circunstelar. Já o inferior mostra a relação entre a mesma largura e a medida de extinção circunstelar $f_{A v}$ (vide texto). Os símbolos cheios representam as fontes PDS com SED com duplo-pico (i.e., distribuição de energia com componentes morna e fria claramente separadas), enquanto que os símbolos vazios indicam distribuições de pico único. Em destaque, encontram-se os objetos PDS 465 e 581, as estrelas pós-AGB já confirmadas desta amostra. 
De forma a verificar a dependência entre a largura equivalente em $\mathrm{H}_{\alpha}$ e o avermelhamento circunstelar, definimos a seguinte grandeza:

$$
f_{A v}=\frac{A_{V t o t}-A_{V}}{A_{V t o t}}
$$

Esta razão representa uma medida da profundidade óptica intrínseca ao material circunstelar, pois leva em consideração o avermelhamento total $A_{V t o t}$, medido a partir do excesso de cor $E(B-V)$ e o $A_{V}$ interestelar, extraído do mapa de extinção visual calculado por Dobashi et al. (2005).

A Figura 2.2 ilustra a dependência da largura equivalente em função desta medida de extinção, apresentando um comportamento análogo àquele observado para $f_{S c} \times \mathrm{W}_{\mathrm{H}_{\alpha}}$. Podemos observar no painel inferior desta figura que os valores de $\mathrm{W}_{\mathrm{H}_{\alpha}}>50 \AA$ são associados à faixa definida por $f_{A v} \gtrsim 0.4$. Este comportamento indica que a intensidade da emissão da linha de $\mathrm{H}_{\alpha}$ está relacionada à extinção intrínseca ao objeto, i.e., associada ao material circunstelar.

\subsection{Diagramas de cores}

Os diagramas de cores podem ser usados como um ótimo diagnóstico para o estudo da natureza ou do status evolutivo de objetos astrofísicos. No trabalho realizado durante o mestrado, desenvolvemos uma extensa análise baseada nesta importante ferramenta (Vieira, 2008), nos mais variados domínios de comprimentos de onda.

Durante o período do doutorado, esta análise foi enriquecida com a inclusão de amostras de objetos com natureza bem conhecida, além da adição de dados observacionais de missões mais recentes (Spitzer, AKARI). Nesta seção pretende-se especificar o refinamento desta análise realizado após a defesa do mestrado.

Os diagramas de cores ópticas e IRAS mantiveram suas versões originais da dissertação 
de mestrado, e portanto omitiremos a sua discussão neste texto (o leitor interessado pode encontrá-los em Vieira et al.|2011). Na faixa do IR, incluímos a informação fornecida pelos fluxos AKARI (Ishihara et al., 2010), disponível para 78\% de nossa amostra de HAeBe. Para tanto, utilizamos o diagrama proposto por Ita et al. (2010), que confronta as cores $J-[18]$ e [9] - [18] (Figura 2.3). Os fluxos e cores AKARI são listados na Tabela 2.2.

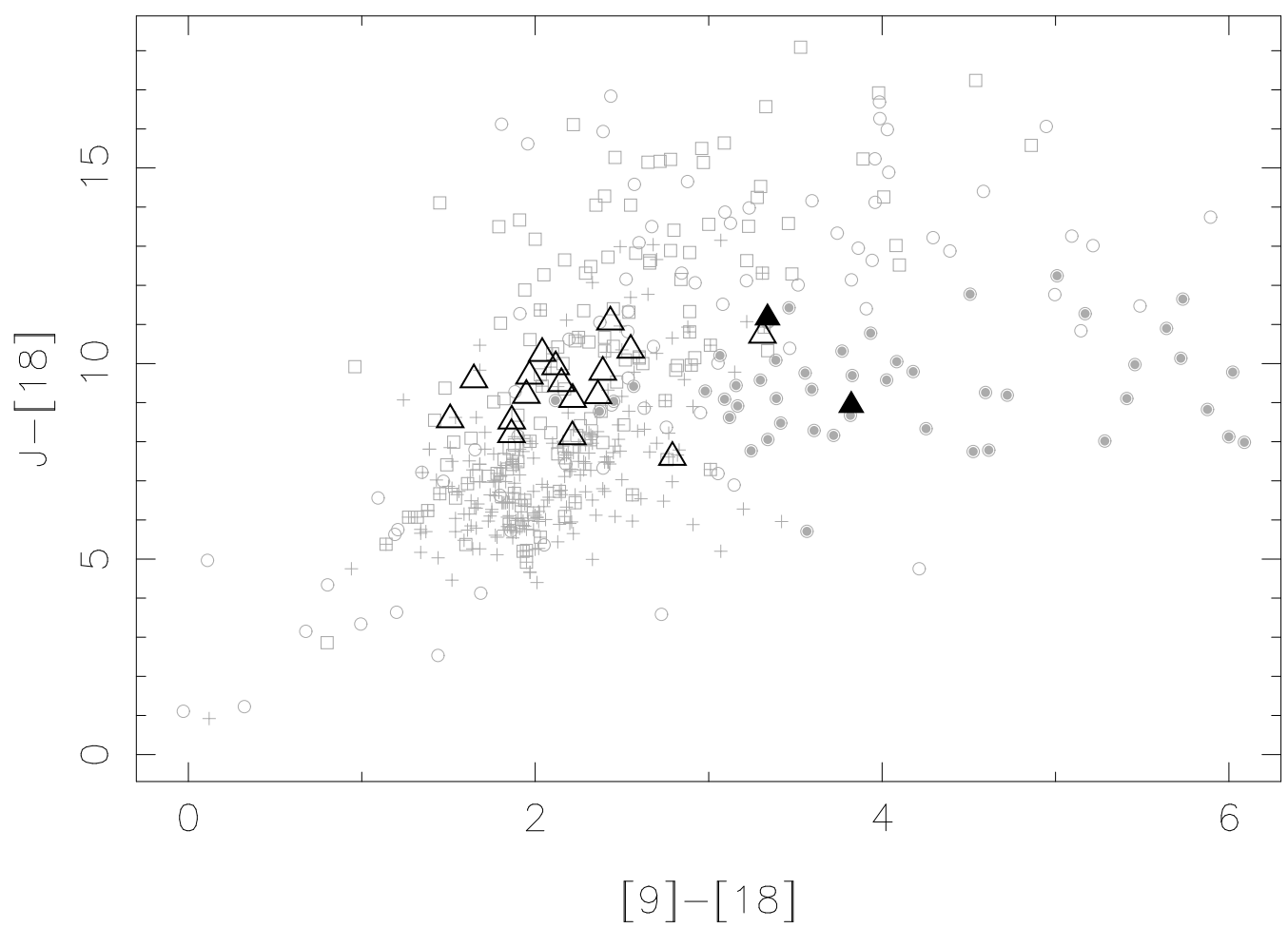

Figura 2.3: Diagrama cor-cor no IR médio, baseado nos fluxos AKARI. Os triângulos indicam os objetos de nossa amostra, enquanto os círculos em cinza representam as very likely pósAGBs do Catálogo de Toruń. Os símbolos preenchidos são usados para os objetos com SED de duplo-pico, enquanto os símbolos abertos representam aqueles com pico único. A distribuição de objetos jovens é representada por quadrados acinzentados (YSOs detectados pelo Spitzer) e cruzes (estrelas HAeBe do HBC).

Tanto neste diagrama, como também para aquele de cores no IR próximo (Vieira, 2008), foram incluídas amostras bem conhecidas de outros trabalhos da literatura. Como representantes da distribuição de objetos que já deixaram a sequência principal, utilizamos aqueles classificados como very likely 1 pós-AGB no Catálogo de Toruń (Szczerba et al.,

\footnotetext{
${ }^{1}$ decidimos aqui manter a nomenclatura em inglês, de forma que ela corresponda exatamente à classificação definida pelo Catálogo de Toruń
} 
Tabela 2.2 - Tabela de fluxos e cores AKARI disponíveis para amostra de candidatas HAeBe mais embebidas do PDS. As cores são expressas em unidades de magnitude Vega, i.e., são calculadas a partir da relação $[\lambda]=-2.5 \times \log \left(F_{\lambda} / F_{V e g a}\right)$.

\begin{tabular}{ccccc}
\hline \hline PDS & $F_{9}$ & $F_{18}$ & $J-[18]$ & {$[9]-[18]$} \\
& Jy & Jy & mag & mag \\
\hline 018 & 13.21 & 19.13 & 10.26 & 2.04 \\
027 & 10.38 & 20.75 & 9.78 & 2.39 \\
037 & 6.06 & 14.06 & 10.34 & 2.55 \\
067 & 7.63 & 6.78 & 8.56 & 1.51 \\
141 & 30.07 & 62.52 & 11.06 & 2.43 \\
168 & 8.11 & 10.00 & 8.53 & 1.87 \\
174 & 27.39 & 29.88 & - & - \\
193 & 2.03 & 2.49 & 8.18 & 1.86 \\
198 & 0.67 & 1.95 & 7.6 & 2.79 \\
204 & 4.44 & 20.72 & 10.73 & 3.31 \\
207 & 3.38 & 5.17 & - & - \\
216 & 1.96 & 1.98 & 9.59 & 1.65 \\
257 & - & 0.55 & - & - \\
290 & - & - & - & - \\
353 & 7.15 & 9.67 & 9.68 & 1.97 \\
371 & 0.28 & 0.55 & 9.18 & 2.36 \\
394 & 1.71 & 8.19 & 11.17 & 3.34 \\
406 & 0.69 & 0.92 & 9.19 & 1.95 \\
431 & - & - & - & - \\
465 & - & 13.56 & 9.73 & - \\
477 & 1.84 & 3.13 & 9.08 & 2.22 \\
518 & - & 218.30 & - & - \\
520 & 1.54 & 2.62 & 8.13 & 2.21 \\
530 & 0.83 & 1.30 & 9.92 & 2.12 \\
543 & 2.78 & 20.73 & 8.93 & 3.82 \\
551 & 1.28 & 2.05 & 9.49 & 2.15 \\
581 & - & 29.03 & 10.82 & - \\
\hline \hline & & & & \\
\hline
\end{tabular}

2007). Os objetos estelares jovens (YSOs) são representados pela lista Spitzer de aglomerados jovens (Gutermuth et al., 2009) e também pelas estrelas HAeBe listadas no Catálogo de Herbig e Bell (HBC - 1988). As distribuições destas amostras são exibidas nas Figuras 2.3 e 2.4

Mesmo a melhor qualidade dos dados AKARI frente aos fluxos IRAS não garantiu que o seu diagrama de cores pudesse distiguir claramente os conjuntos de objetos confrontados. 
A distribuição das várias amostras ainda apresenta uma grande superposição, a qual nos impede de classificar o status evolutivo dos objetos PDS apenas com este critério de cor. No entanto, ainda assim podemos destacar os três objetos PDS 204, 394 e 543 no diagrama que se encontram na faixa $[9]-[18]>3$, separados da região tipicamente ocupada pelas outras HAeBe. Este comportamento pode representar um indício de uma natureza mais evoluída destes objetos.

Na Figura 2.4, são apresentadas as regiões definidas por Garcia-Lario et al. (1997). As Regiões I e II são ocupadas tipicamente por objetos de sequência principal, enquanto as Regiões III e IV por estrelas T Tauri e HAeBe. A Região III também apresenta uma grande incidência de YSOs, e a Região V representa o locus típico de nebulosas planetárias.

Além das amostras de natureza conhecida, foram incluídas algumas informações extras em uma nova versão do diagrama de cores no IR próximo. A área delimitada pela curva fechada tracejada abriga tipicamente objetos ricos em carbono, enquanto que as estrelas variáveis de longo período ricas em oxigênio estão distribuídas dentro da curva fechada contínua (Bessell e Brett, 1988). Apenas o objeto PDS 551 se mostrou estar contido na região povoada por objetos ricos em carbono.

Podemos notar que vários de nossos objetos são encontrados na mesma região tipicamente ocupada por estrelas pós-AGB com SED de pico único, enquanto os objetos de duplo pico povoam a Região I. Siódmiak et al. (2008) verificaram em seu trabalho com pós-AGBs uma clara separação entre as classes morfológicas SOLE (Star-Obvious Lowlevel Elongated) e DUPLEX (DUst-Prominent Longitudinally-EXtended) no diagrama de cores no IR próximo (Ueta et al., 2000, 2007). Objetos do tipo SOLE possuem uma camada opticamente fina de formato elíptico e ocupam a região definida por $H-K<0.75$ e $J-H<1$. Os do tipo DUPLEX apresentam uma geometria bipolar com a presença de um toro circunstelar opticamente espesso, e são encontrados em $J-H>1$. O locus de objetos de tipo estelar definidos por Siódmiak et al. (2008) coincide com a Região IV delineada por Garcia-Lario et al. (1997), a qual conforme já mencionado é normalmente ocupada por estrelas T Tauri e HAeBe. 


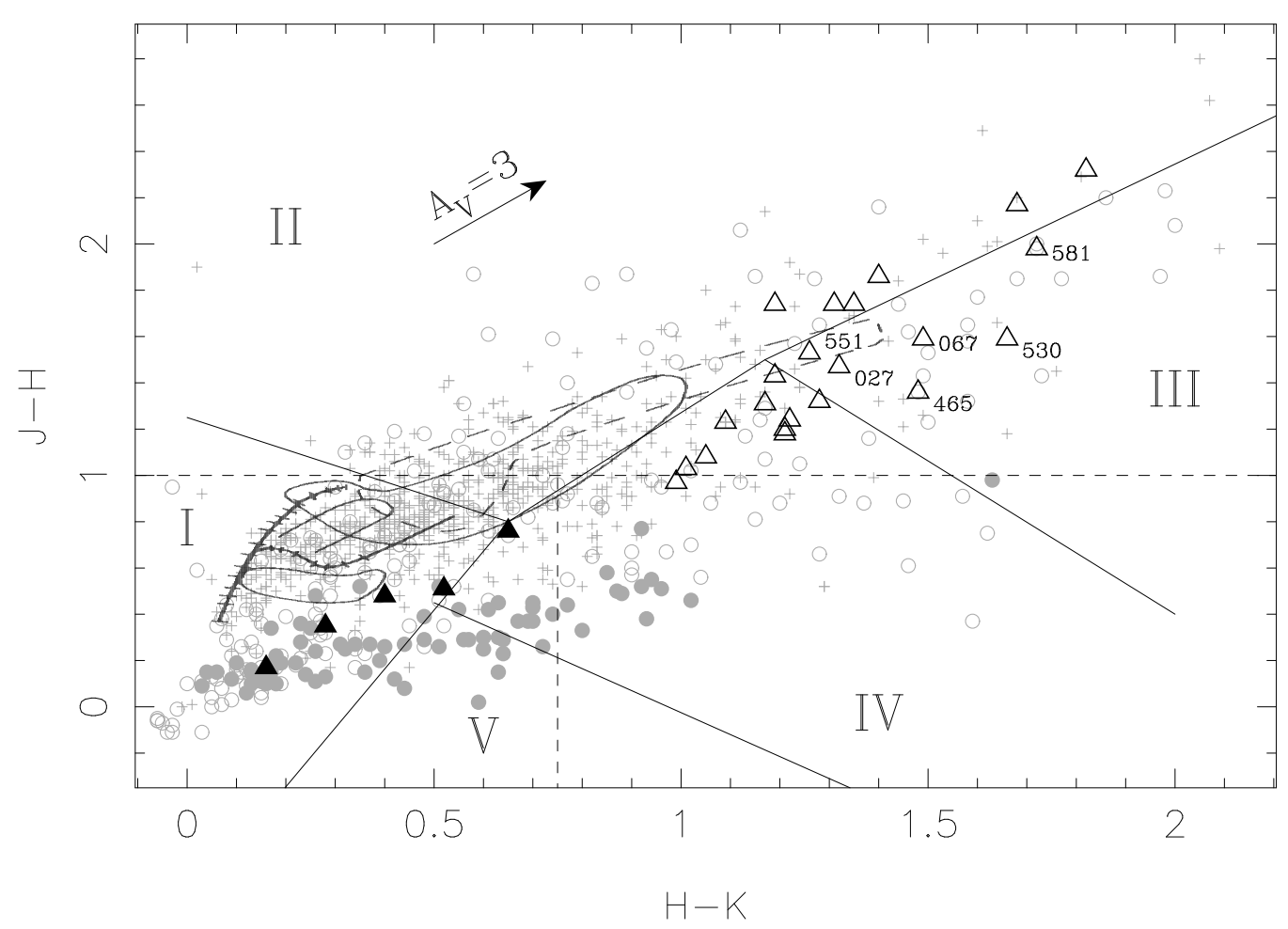

Figura 2.4: Diagrama de cor no IR próximo, indicando as regiões (I a V) associadas às categorias de objetos definidas por Garcia-Lario et al. (1997, separadas por linhas cheias), Siódmiak et al. (2008, linhas tracejadas finas) e por Bessell e Brett (1988, curvas fechadas). Os triângulos representam objetos de nossa amostra e os círculos em cinza indicam as pósAGB very likely do Catálogo de Toruń. Objetos pós-AGB com SED de duplo-pico são representados por círculos cheios, pós-AGB com SED de pico único por círculos vazios e as cruzes indicam os objetos HAeBe do HBC. Para maiores detalhes, vide texto.

Finalmente, incluímos na análise o estudo da distribuição de nossa amostra no diagrama de cores $J-K$ vs. $K-$ [25] (Figura 2.5). Ueta et al. (2000, 2007) mostraram que a posição neste diagrama é correlacionada à morfologia dos objetos estudados por eles. $\mathrm{O}$ trabalho destes autores nos mostra que podemos encontrar as pós-AGB de tipo estelar na região à esquerda $(K-[25]<8)$, enquanto que a porção da direita do diagrama é divida pelos objetos do tipo SOLE (parte inferior) e DUPLEX (parte superior).

O formato da SED também é correlacionado a esta classificação, onde os objetos que apresentam duplo-pico são do tipo SOLE e os de pico simples DUPLEX. Siódmiak et al. (2008) argumentam que uma distribuição de energia de duplo-pico é compatível com a 


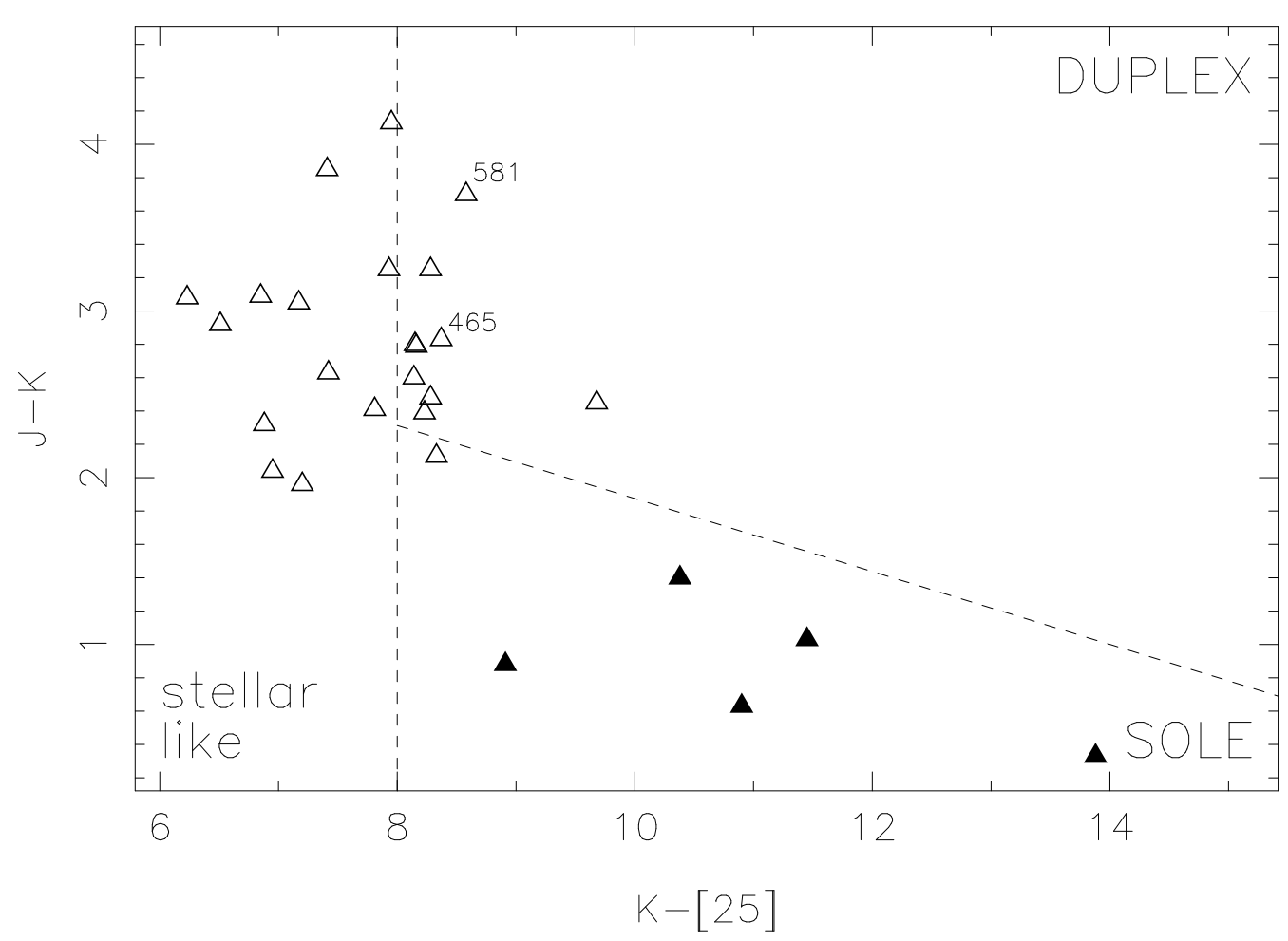

Figura 2.5: Diagrama de cores no IR próximo e médio indicando as regiões definidas por Siódmiak et al. (2008) (linhas tracejadas). Triângulos preenchidos indicam as estrelas de nossa amostra com SED em duplo-pico, enquanto triângulos abertos representam aquelas com pico único.

emissão de uma fonte central mais uma componente circunstelar elipsoidal, enquanto que uma SED de pico único pode ser explicada por meio da alta extinção causada pela presença de um toro de poeira ao redor do objeto central. O diagrama de cores apresentado na Figura 2.5 mostra que esta correlação também é obedecida pelos objetos de nossa amostra de candidatas HAeBe, já que a distribuição de fontes com SED de duplo-pico coincide com aquela definida para a classe SOLE.

No entanto, apesar da boa concordância entre o formato das SEDs e a posição neste diagrama de cores, nada podemos afirmar sobre a morfologia de nossos objetos, dada a ausência de observações de imagens diretas que a comprovem. A única exceção a ser feita é o caso de PDS 204, o qual possui imagens no IR e dados polarimétricos que confirmam sua geometria bipolar (Perrin et al., 2009). Neste caso, a classificação como um objeto do tipo DUPLEX feita a partir das cores IR deste objeto são coerentes com esta informação. 


\subsection{Refinamento da amostra}

De acordo com os critérios expressos na Tabela 2 de Vieira et al. (2011, Apêndice B]), os objetos mais prováveis de se encontrarem na fase de pós-AGB são PDS 174, 204, 290, 371, 465, 543 e 581, que foram destacados como objetos de comportamento mais incompatível com uma natureza jovem (Vieira et al., 2011). Entre tais objetos, encontram-se PDS465 e PDS581, cuja natureza pós-AGB é confirmada na literatura (Riera et al. 1995; Bowers e Knapp 1989; Rodrigues et al. 2003).

De maneira similar à empregada para apontar as estrelas já provavelmente na fase de pós-AGB, podemos também indicar os objetos com mais características de estrelas jovens (i.e., com um menor número de "notas" na Tabela 2 de Vieira et al. 2011). Estes objetos são PDS 67, 193, 477, 530 e 543, representando portanto as candidatas HAeBe estudadas por nós como as mais provavelmente no estágio de PMS. 
Capítulo 3

\section{Química da poeira circunstelar}

Discos protoplanetários possuem cerca de três quartos de sua massa sob a forma de $\mathrm{H}_{2}$ e um quarto formado por He gasoso. Há ainda uma numerosa lista de moléculas que os constituem, sendo os exemplos mais comuns as moléculas de $\mathrm{CO}, \mathrm{H}_{2} \mathrm{O}$ e $\mathrm{O}_{2}$ (e.g., Kamp e Bertoldi 2000). Entre as maiores moléculas presentes nestes ambientes, encontramos os hidrocarbonetos policíclicos aromáticos (PAHs). Por sua vez, os PAHs são formados por aneis de benzeno conectados de formas variadas.

Apesar da composição do material circunstelar ser dominada por moléculas, os grãos de poeira são os maiores responsáveis pela opacidade observada nestes meios. Representando apenas $\sim 1 \%$ da massa destas estruturas, a poeira absorve e re-emite a radiação da fonte central do sistema, dando origem ao excesso de emissão infravermelha (IR) associado a estrelas jovens. Os grãos de poeira são compostos principalmente por silicatos. Cerca de $15 \%$ de sua composição é constituída por carbonáceos (carbono amorfo, grafite e nanodiamantes) e entre $10 \%$ e $15 \%$ é formada por sulfureto de ferro e ferro metálico (Verhoeff, 2009).

Todos os silicatos contêm oxigênio e silício. Seus outros constituintes determinam a sua classificação. A olivina por exemplo tem a fórmula $\mathrm{Mg}_{2 \mathrm{x}} \mathrm{Fe}_{2-2 \mathrm{x}} \mathrm{SiO}_{4}$, com $0 \leq x \leq 1$. O piroxeno é representado por $\mathrm{Mg}_{\mathrm{x}} \mathrm{Fe}_{1-\mathrm{x}} \mathrm{SiO}_{3}$, e a silica por $\mathrm{SiO}_{2}$. A forsterita $\left(\mathrm{Mg}_{2} \mathrm{SiO}_{4}\right)$ é o membro extremo das olivinas $(x=1)$, enquanto que a enstatita $\left(\mathrm{MgSiO}_{3}\right)$ possui a máxima fração de magnésio entre os piroxenos. A Figura 3.1 exibe os silicatos mais comuns encontrados nos ambientes circunstelares. Quando os grupos de silicatos estão 

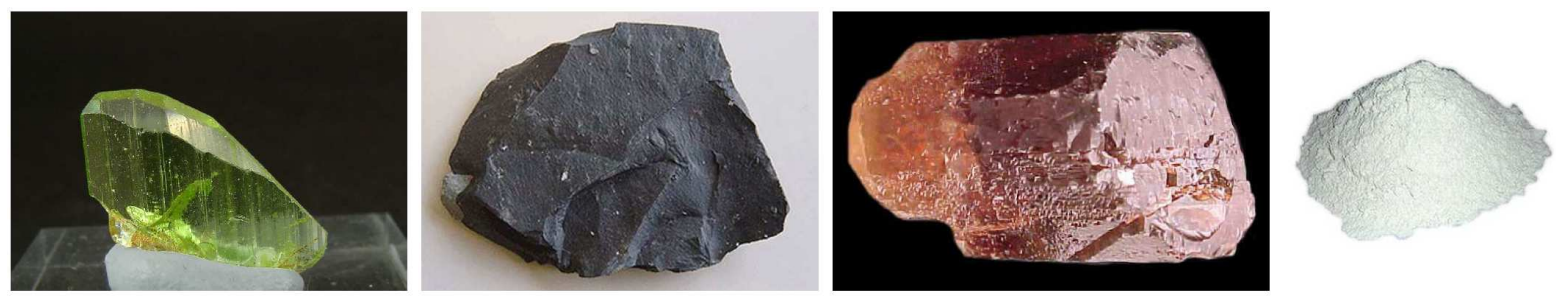

Figura 3.1: Minerais tipicamente observados em discos protoplanetários. Da esquerda para direita: olivina, piroxeno, enstatita e sílica. Figura adaptada de Verhoeff (2009).

orientados aleatoriamente em sua estrutura sólida, eles são ditos amorfos. O aquecimento deste material a temperaturas superiores a $\sim 1000 \mathrm{~K}$ leva ao alinhamento de sua estrutura, tornando-o cristalino.

Vários trabalhos na literatura apontam para a evolução dos grãos de poeira em discos circunstelares (Bouwman et al. 2001;vvan Boekel et al. 2005; Kessler-Silacci et al. 2005). O material presente no meio interestelar (ISM), origem primária da massa contida em sítios de formação estelar, é constituído principalmente de olivina (Whittet et al., 1997). A incidência de raios cósmicos tende a manter a natureza amorfa deste material (Bringa et al., 2007). No entanto, observações realizadas por Bowey e Adamson (2002) sugerem uma transição de olivinas para piroxenos em nuvens moleculares e objetos estelares jovens (YSOs). A presença de material cristalino em ambientes circunstelares em estágios mais avançados evidenciam a evolução dos grãos de poeira (e.g., Knacke et al. 1993). Através do estudo da região espectral em torno de $10 \mu \mathrm{m}$ de uma amostra de estrelas Herbig Ae/Be (HAeBe), Bouwman et al. (2005) estudaram de forma sistemática a evolução da poeira circunstelar. Este trabalho mostra que o deslocamento do pico de emissão do silicato, de $9.7 \mu \mathrm{m}$ para $11.3 \mu \mathrm{m}$, é devido especialmente ao aumento do tamanho médio dos grãos, sendo as mudanças químicas menos importantes. O mesmo estudo também sugere que as estrelas HAeBe, o objeto $\beta$ Pictoris e o cometa Halley formam uma sequência em cristalização. Esta assertiva é consistente com o trabalho de Kemper et al. (2004), que mostra que a fração cristalina de cometas (de até 30\%) é muito maior do que aquela encontrada no $\operatorname{ISM}(<2 \%)$. A Figura 3.2 ilustra a evolução do silicato sugerida por Bouwman et al. (2001). 

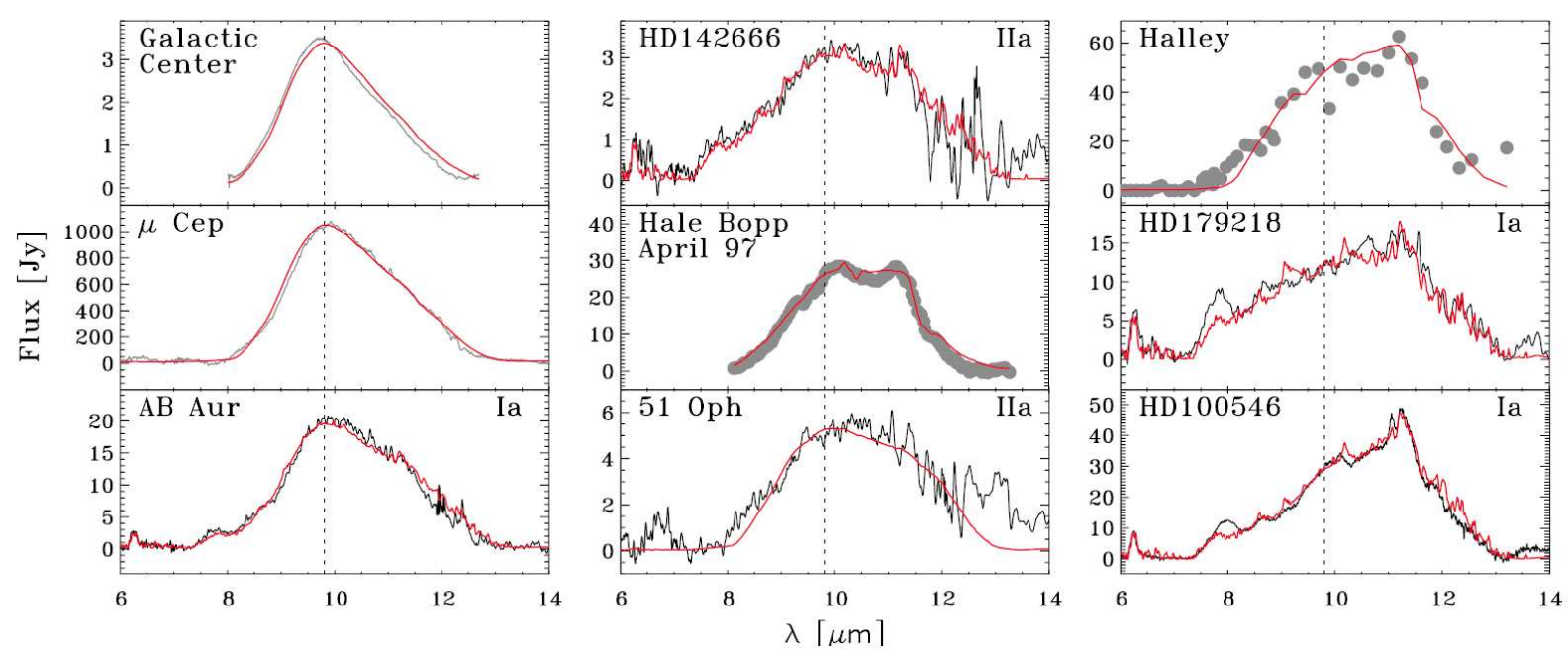

Figura 3.2: Evolução do perfil de emissão do silicato, observada em torno de $10 \mu \mathrm{m}$ em espectros ISO. Além de objetos HAeBe, também estão representados os espectros do meio interestelar na direção do centro da Galáxia, a super-gigante vermelha $\mu$ Cep e os cometas Hale Bopp e Halley. A sequência sugere que o grau de cristalização aumenta com a evolução do disco. Figura adaptada de Bouwman et al. (2001).

Kessler-Silacci et al. (2005 - KS05 daqui em diante) realizaram um estudo de 34 objetos jovens, utilizando observações feitas com o telescópio W. M. Keck entre $8 \mu \mathrm{m}$ e $13 \mu \mathrm{m}$. Estes autores analisaram a dependência da intensidade da emissão do silicato com a inclinação da distribuição espectral de energia (SED), mostrando que a assinatura espectral do silicato evolui de um perfil de absorção nos objetos mais embebidos a um perfil de emissão nos objetos já visíveis em bandas ópticas.

Neste capítulo estudaremos as características da poeira reveladas por espectros ISO SWS e IRAS LRS disponíveis para as estrelas HAeBe do PDS. A próxima seção descreverá a seleção de nossa amostra e sua classificação de acordo com as características espectrais. A Seção 3.2 estenderá a metodologia empírica empregada por KS05 a estes objetos. Finalmente, a Seção 3.3 apresentará o estudo das abundâncias das componentes de poeira para o caso específico do objeto PDS061, feito com o código DUSTY (Ivezic e Elitzur, 1997) e a implementação de algoritmos genéticos. 


\subsection{Sub-amostra PDS}

Nossa amostra é constituida basicamente dos objetos HAeBe detectados pelo PDS que dispõem de observações ISO (Infrared Space Observatory - Jourdain de Muizon (2005)) obtidas com o espectrômetro de comprimentos de onda curtos (SWS1 - Short Wavelength Spectrometer). Este instrumento possui uma cobertura espectral entre $2.38 \mu \mathrm{m}$ e $45.2 \mu \mathrm{m}$, revelando as características da emissão de poeira não apenas associada a estrelas jovens, como também do ISM e de outras galáxias.

As observações disponíveis na base de dados do catálogo IRAS LRS (Low Resolution Spectra) também foram utilizadas, na ausência de espectros ISO. Estas observações cobrem o intervalo espectral entre $8 \mu \mathrm{m}$ e $22 \mu \mathrm{m}$, compreendendo os objetos mais brilhantes detectados pela missão IRAS. A Tabela 3.1 lista os objetos HAeBe do PDS com observações ISO SWS e IRAS LRS. Foram excluídos desta amostra os objetos PDS465 e PDS581, por se tratarem de estrelas pós-AGB já confirmadas pela literatura (Riera et al. 1995; Bowers e Knapp 1989; Rodrigues et al. 2003). Apesar da análise de Vieira et al. (2011) ter indicado PDS174 como um possível objeto pós-AGB (Capítulo 2), decidimos mantê-lo no presente estudo, dado que a confirmação de sua classificação se dará apenas por meio de novas observações.

Os espectros ISO SWS disponíveis são exibidos na Figura 3.3 (espectros IRAS LRS serão apresentados na próxima seção), onde também destacamos a faixa ocupada pelo perfil de silicato. Os espectros foram multiplicados por fatores arbitrários (que se traduzem em deslocamentos aditivos em escala logarítmica), de modo que possamos estabelecer comparações entre os objetos. Esta representação permitiu a classificação da amostra segundo os critérios de Meeus et al. (2001). Tanto estas classes como a separação em grupos feita previamente por Sartori et al. (2010) são apresentadas na Tabela $3.12^{2}$. Finalmente, apresentamos também os fluxos IRAS em $12 \mu \mathrm{m}$, para que conheçamos os valores absolutos de fluxo no IR de cada objeto.

\footnotetext{
${ }^{1}$ mais especificamente, os espectros SWS01 da base do dados do-ISO.

${ }^{2}$ As classificações de Meeus et al. (2001) e de Sartori et al. (2010) encontram-se descritas no Capítulo 1
} 
Tabela 3.1 - Objetos HAeBe do PDS com espectro ISO SWS ou IRAS LRS.

\begin{tabular}{llcccc}
\hline \hline PDS & Observação & $F_{12}$ & Perfil do Silicato & \multicolumn{2}{c}{ Grupo } \\
& & $(\mathrm{Jy})$ & & Sartori et al. & Meeus et al. \\
\hline 018 & IRAS 05513-1024 & 17.50 & ausente & 1 & - \\
061 & ISO 23300524 & 23.65 & emissão & 2 & IIa \\
076 & ISO 44901283 & 8.57 & emissão & 2 & IIa \\
078 & ISO 45000284 & 7.53 & emissão & 2 & IIa \\
141 & IRAS 12496-7650 & 39.32 & absorção & 1 & - \\
172 & ISO 83501201 & 10.25 & emissão & 2 & IIa \\
174 & IRAS 05044-0325 & 13.10 & absorção & 1 & - \\
176 & ISO 83301240 & 0.70 & superposição? & 2 & IIb \\
339 & ISO 26000230 & 7.23 & ausente & 2 & Ib \\
340 & ISO 07200660 & 65.78 & emissão & 2 & Ia \\
395 & ISO 29701542 & 4.11 & ausente & 2 & Ib \\
473 & ISO 32901191 & 18.20 & emissão & 2 & IIa \\
514 & ISO 13601359 & 2.95 & superposição? & 2 & Ib \\
518 & ISO 70800234 & 158.70 & absorção & 1 & IIa \\
564 & ISO 32301321 & 23.44 & emissão & 2 & Ia \\
\hline \hline
\end{tabular}




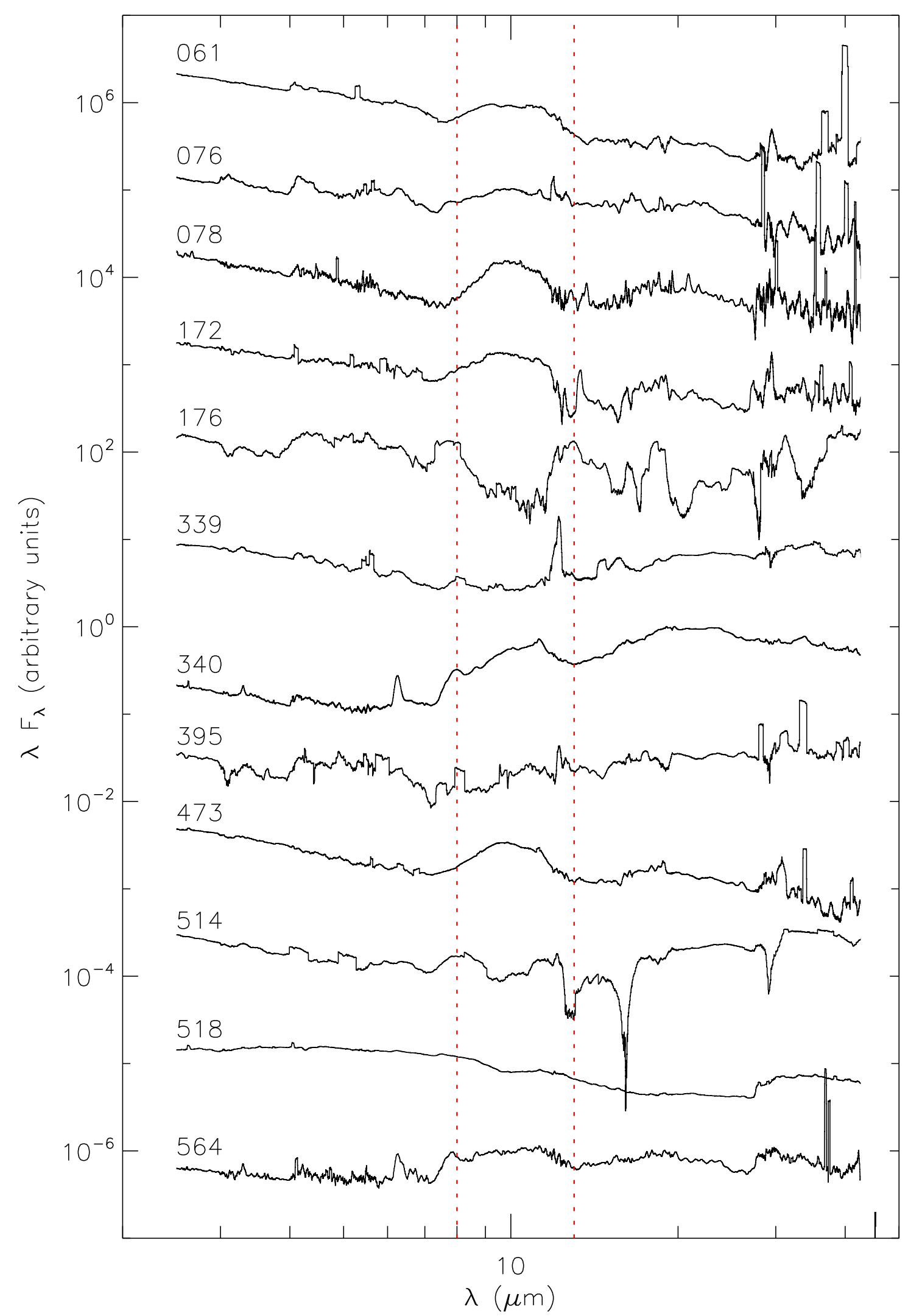

Figura 3.3: Espectros ISO disponíveis para objetos HAeBe do PDS, multiplicados por fatores arbitrários. A faixa espectral ocupada pelo perfil de silicato é destacada entre as linhas tracejadas em vermelho. 


\subsection{O perfil do silicato}

A compreensão do perfil de emissão/absorção do silicato depende do conhecimento detalhado da opacidade do envoltório circunstelar e da estrutura vertical do disco de poeira. Como nossa amostra contém uma grande variedade de estruturas circunstelares, que abrange desde objetos mais embebidos do Grupo 1 do PDS (Sartori et al., 2010) aos mais evoluídos do Grupo 2, decidimos adotar a abordagem empírica apresentada pelo trabalho de KS05.

\subsubsection{Comparação: ISO × Keck}

Antes de extrair as informações necessárias para nossa análise dos espectros disponíveis, decidimos comparar os resultados obtidos por meio de dados ISO (utilizados neste trabalho) e observações Keck (KS05). Para tanto, buscamos os espectros ISO referentes à amostra utilizada por KS05.

Infelizmente, apenas cinco entre os 34 objetos de KS05 foram encontrados na base de dados ISO. Seus espectros ISO (normalizados pelo contínuo) são exibidos na Figura 3.4, ao lado das observações Keck de KS05.

O primeiro passo para desenvolvermos nossa análise foi a normalização dos espectros, segundo a expressão:

$$
F_{\text {norm }}=\frac{F_{\nu}-F_{c}}{F_{c}}
$$

A contribuição do contínuo foi estimada a partir do ajuste linear realizado entre $8.3 \mu \mathrm{m}$ e $12.2 \mu \mathrm{m}$, de acordo com o procedimento descrito por KS05. Para todos os valores de fluxo, tomamos a média dentro de um intervalo de $0.2 \mu \mathrm{m}$ em torno do valor central, sendo a incerteza da medida estimada pelo desvio padrão do fluxo nesta região. No caso das observações IRAS LRS, a grade de comprimentos de onda é mais esparsa do que nos espectros ISO, o que tira o sentido da medida da dispersão dentro de $0.2 \mu \mathrm{m}$. Por 

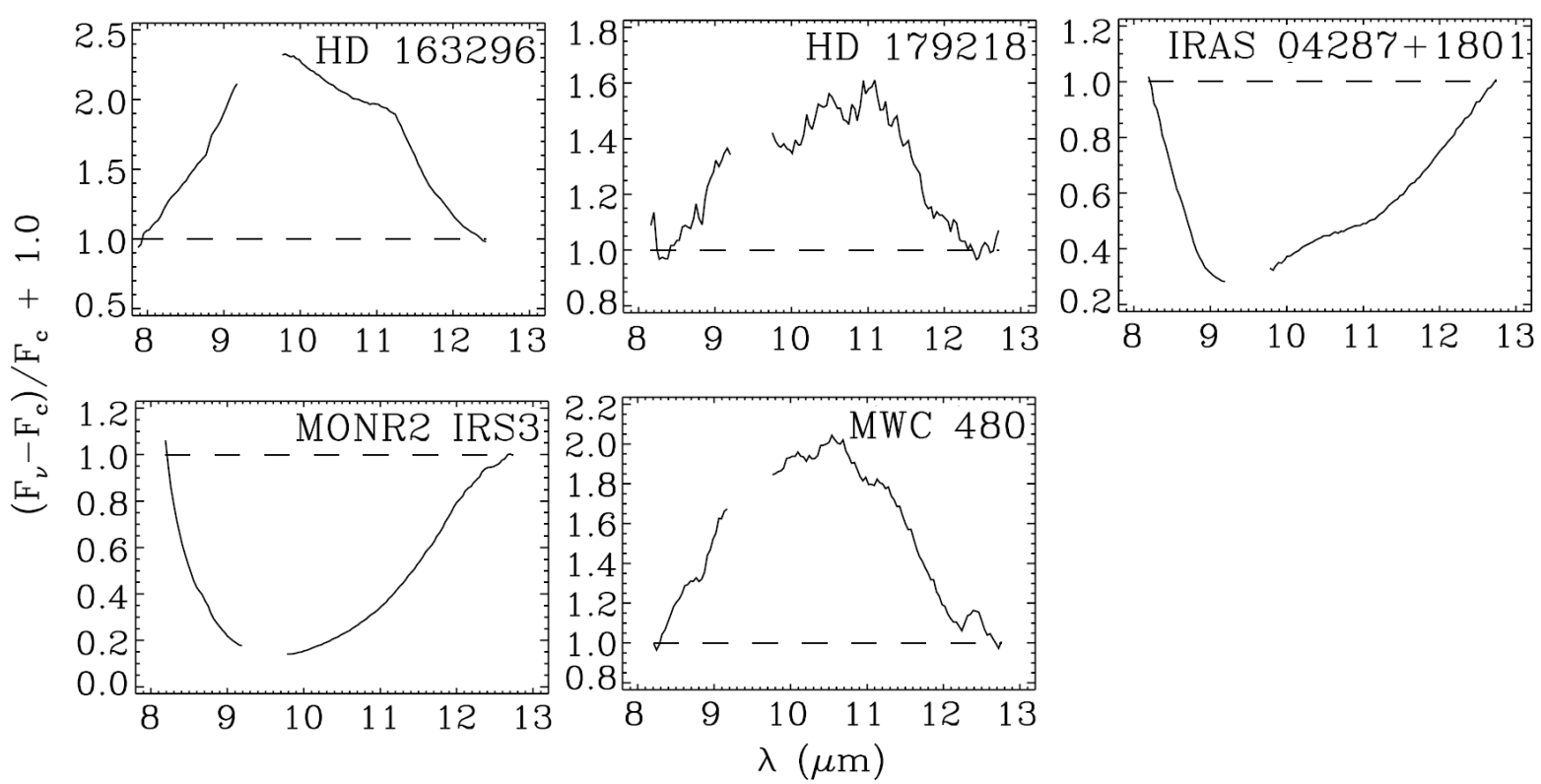

(a) Espectros Keck de KS05.
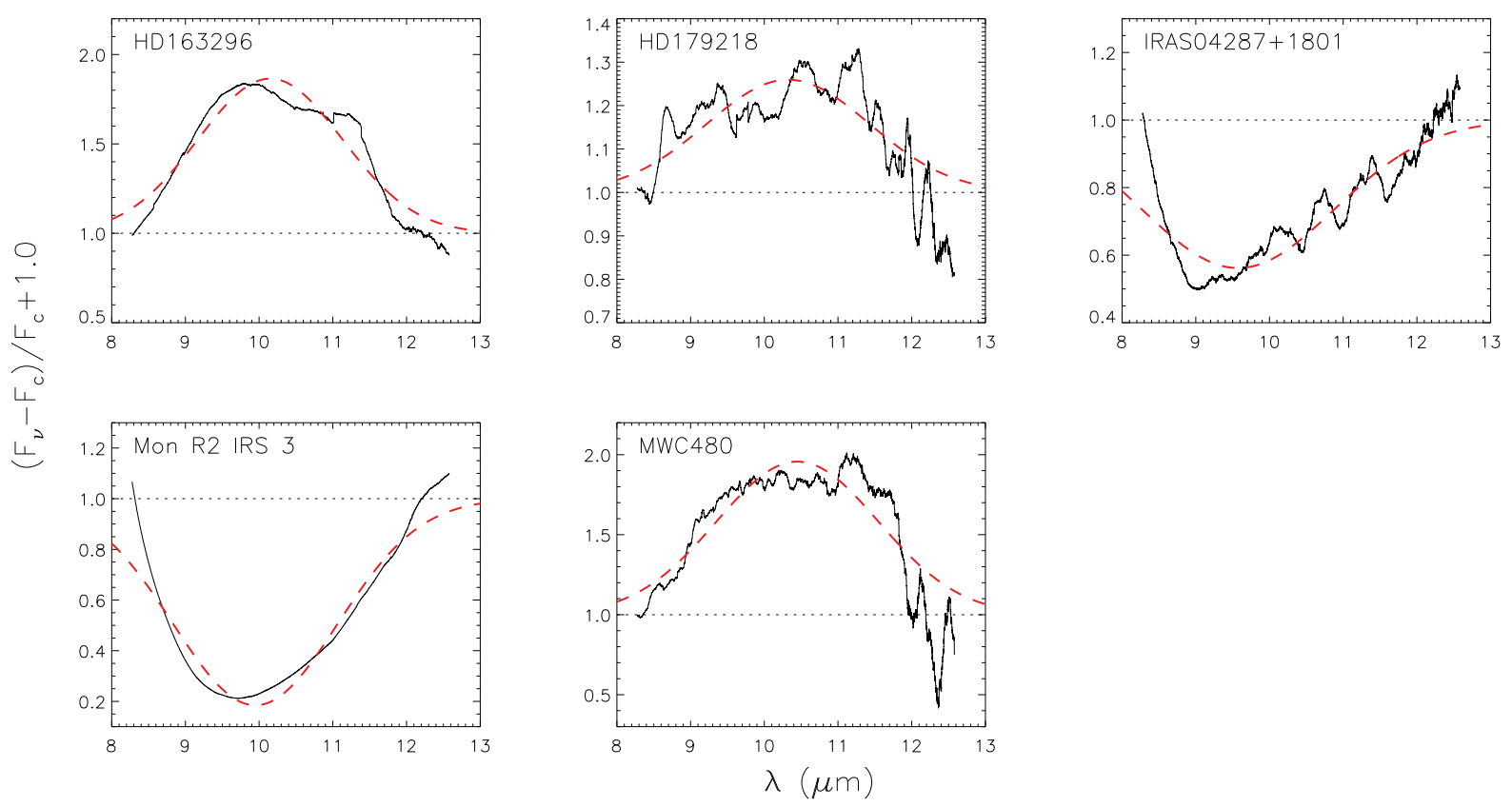

(b) Espectros ISO de sub-amostra de KS05.

Figura 3.4: O painel superior exibe os espectros obtidos por KS05 com o telescópio Keck. O painel inferior mostra a mesma sub-amostra, observada pela missão ISO. As linhas vermelhas tracejadas representam os ajustes gaussianos calculados calculados no presente trabalho. 
isto para estes objetos estimamos a incerteza na medida do fluxo a partir da expressão $\delta F=\max \left(\left|F_{j}-F_{j-1}\right|,\left|F_{j}-F_{j+1}\right|\right)$, sendo $F_{j}$ o fluxo (normalizado) na posição mais próxima da desejada. Estes valores se mostraram superiores ao erro percentual do fluxo IRAS em $12 \mu \mathrm{m}$, por cerca de um fator 2 .

A Tabela 3.2 exibe os valores apresentados por KS05 e aqueles calculados a partir dos espectros ISO. Nela, são apresentadas as posições centrais do ajuste gaussiano $\left(\lambda_{1}\right)$ e a posição de máximo (ou mínimo) do perfil de silicato $\left(\lambda_{2}\right)$. Os respectivos fluxos medidos em cada uma destas posições, $F_{\lambda_{1}}$ e $F_{\lambda_{2}}$, também são exibidos na tabela. As estimativas de incerteza destas medidas seguem o procedimento análogo ao descrito para a determinação do contínuo. A largura a meia altura, FWHM, representa o valor calculado pelo ajuste gaussiano. Para os valores de $\lambda_{1}, \lambda_{2}$ e FWHM, estimamos uma incerteza de $\pm 0.1 \mu \mathrm{m}$, devido à média dentro de $0.2 \mu \mathrm{m}$ utilizada nos pontos que determinam o contínuo. A Figura 3.5 confronta diretamente as medidas de KS05 com as nossas estimativas. Nesta comparação, quantificamos a posição de pico através da grandeza $\lambda_{1,2}=0.5 \times\left[\left(\lambda_{1}+\lambda_{2}\right) \pm\left|\lambda_{1}-\lambda_{2}\right|\right]$. Esta definição permite a estimativa do erro associado à medida da posição de máximo (ou mínimo).

Tabela 3.2 - Medidas do perfil de silicato dos objetos de nossa amostra. Os valores de $\lambda_{1}$ e $F_{\lambda_{1}}$ são respectivamente as estimativas da posição central do ajuste gaussiano e a sua respectiva intensidade. $\lambda_{2}$ e $F_{\lambda_{2}}$ representam a posição de máximo (ou mínimo) do perfil de silicato e a intensidade nesta posição.

\begin{tabular}{lccccc|cccccc}
\hline \hline \multicolumn{1}{c}{ Dados Keck } \\
\hline & $\lambda_{1}$ & $\lambda_{2}$ & $F_{\lambda_{1}}$ & $F_{\lambda_{2}}$ & FWHM & $\lambda_{1}$ & $\lambda_{2}$ & $F_{\lambda_{1}}$ & $F_{\lambda_{2}}$ & $\begin{array}{c}\text { FWHM } \\
(\text { (normalizado) }\end{array}$ & $(\mu \mathrm{m})$ \\
\hline HD163296 & 9.61 & 10.02 & 1.33 & 1.34 & 2.48 & 10.15 & 9.85 & $0.86 \pm 0.21$ & $0.81 \pm 0.24$ & 2.31 \\
HD179218 & 11.01 & 10.48 & 0.56 & 0.53 & 2.39 & 10.32 & 11.22 & $0.26 \pm 0.20$ & $0.31 \pm 0.35$ & 2.61 \\
IRAS 04287+1801 & 9.23 & 9.93 & -0.71 & -0.69 & 3.17 & 9.57 & 9.02 & $-0.44 \pm 0.36$ & $-0.49 \pm 0.41$ & 3.06 \\
Mon R2 IRS 3 & 9.82 & 9.93 & -0.85 & -0.90 & 2.93 & 9.95 & 9.76 & $-0.82 \pm 0.03$ & $-0.78 \pm 0.02$ & 2.62 \\
MWC 480 & 10.58 & 10.32 & 1.00 & 1.02 & 2.45 & 10.45 & 11.08 & $0.96 \pm 0.45$ & $0.94 \pm 0.61$ & 2.60 \\
\hline \hline
\end{tabular}



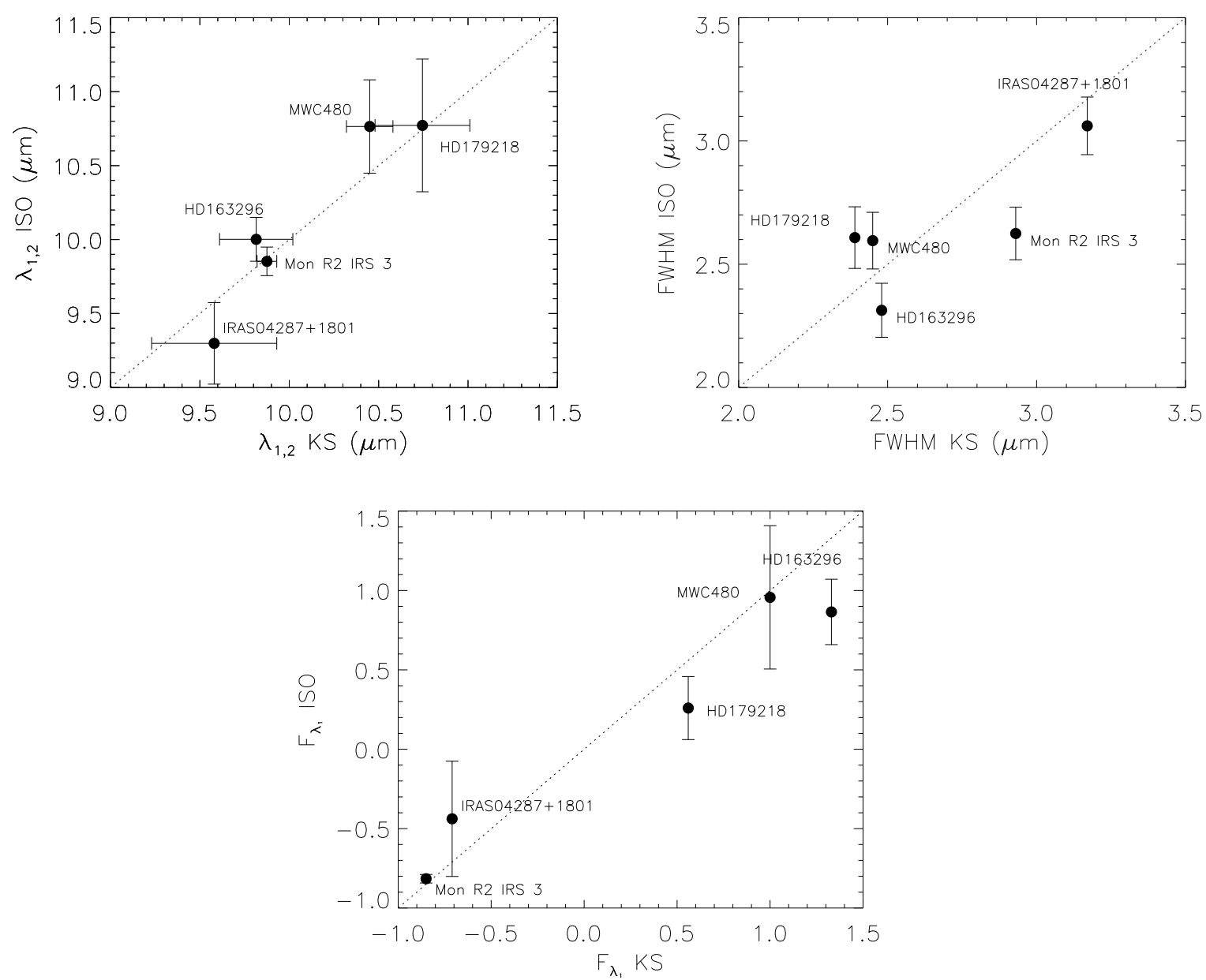

Figura 3.5: Comparação entre grandezas calculadas por KS05 e aquelas calculadas a partir dos espectros ISO. O painel superior à esquerda exibe os valores de $\lambda_{1,2}$ (vide definição no texto). O gráfico superior à direita representa os valores da largura à meia altura, enquanto que o painel inferior mostra os valores da intensidade dos espectros normalizados. Tanto FWHM como $F_{\lambda_{1}}$ são grandezas determinadas pelo ajuste gaussiano aos perfis de emissão/absorção. A linha pontilhada representa a concordância ideal entre as medidas.

A comparação entre os conjuntos de resultados indica que as estimativas realizadas a partir de espectros ISO são suficientemente satisfatórias para nossos propósitos. Obviamente, apenas uma amostra mais numerosa poderia confirmar a equivalência entre os resultados com real significância estatística. No entanto, se tomarmos como base a comparação estabelecida, podemos argumentar simplesmente que os eventuais desvios observados não alterariam as conclusões discutidas posteriormente. 


\subsubsection{Resultados}

As medidas realizadas para nossa amostra podem ser encontradas na Tabela 3.3, Os espectros normalizados são apresentados na Figura 3.6, sobre os quais são sobrepostos os ajustes gaussianos calculados.

Tabela 3.3 - Medidas do perfil de silicato dos objetos de nossa amostra. Os valores listados são os mesmos descritos no cabeçálio da Tabela 3.2 A última coluna apresenta o valor do índice espectral entre $2.2 \mu \mathrm{m}$ (banda $K_{S}$ 2MASS) e $25 \mu \mathrm{m}$ (IRAS).

\begin{tabular}{|c|c|c|c|c|c|c|}
\hline \multicolumn{3}{|c|}{$(\mu \mathrm{m})$} & $\begin{array}{l}F_{\lambda_{1}} \\
\quad(\text { norm }\end{array}$ & $F_{\lambda_{2}}$ & $\begin{array}{c}\text { FWHM } \\
(\mu \mathrm{m})\end{array}$ & $\alpha_{2-25}$ \\
\hline 018 & - & - & - & - & - & -0.14 \\
\hline 061 & 10.20 & 10.73 & $0.34 \pm 0.27$ & $0.37 \pm 0.97$ & 2.78 & 0.65 \\
\hline 076 & 9.82 & 9.75 & $0.25 \pm 0.40$ & $0.25 \pm 0.38$ & 1.35 & 0.38 \\
\hline 078 & 10.08 & 10.41 & $1.29 \pm 0.91$ & $1.18 \pm 1.76$ & 2.14 & 0.53 \\
\hline 141 & 9.95 & 9.97 & $-0.32 \pm 0.03$ & $-0.32 \pm 0.03$ & 2.02 & -0.11 \\
\hline 172 & 10.45 & 11.08 & $0.96 \pm 0.45$ & $0.94 \pm 0.61$ & 2.60 & 0.62 \\
\hline 174 & 10.14 & 10.39 & $-0.35 \pm 0.02$ & $-0.34 \pm 0.03$ & 1.43 & -1.03 \\
\hline 176 & - & - & - & - & - & 0.09 \\
\hline 339 & - & - & - & - & - & 0.57 \\
\hline 340 & 10.54 & 11.22 & $0.63 \pm 0.14$ & $0.76 \pm 0.15$ & 2.30 & -0.63 \\
\hline 395 & - & - & - & - & - & -0.07 \\
\hline 473 & 10.15 & 11.21 & $0.86 \pm 0.21$ & $0.71 \pm 1.66$ & 2.31 & 0.61 \\
\hline 514 & - & - & - & - & - & 0.05 \\
\hline 518 & 9.82 & 9.60 & $-0.17 \pm 0.07$ & $-0.18 \pm 0.04$ & 1.51 & 0.30 \\
\hline 564 & 10.32 & 11.25 & $0.26 \pm 0.20$ & $0.30 \pm 0.38$ & 2.61 & -0.14 \\
\hline
\end{tabular}

A última coluna da Tabela 3.3 também apresenta os valores do índice espectral IR, definido por Lada (1987). Esta grandeza é diretamente relacionada à distribuição do material circunstelar, revelando portanto as características do ambiente onde o perfil do silicato foi originado. A definição de $\alpha_{2-25}$ depende das medidas de fluxo na banda $K_{S}$ (2MASS) e em $25 \mu \mathrm{m}$ (IRAS), sendo expressa por:

$$
\alpha_{2-25}=-\frac{\log \left(25 \times F_{25}\right)-\log \left(2.2 \times F_{K}\right)}{\log (25)-\log (2.2)} .
$$



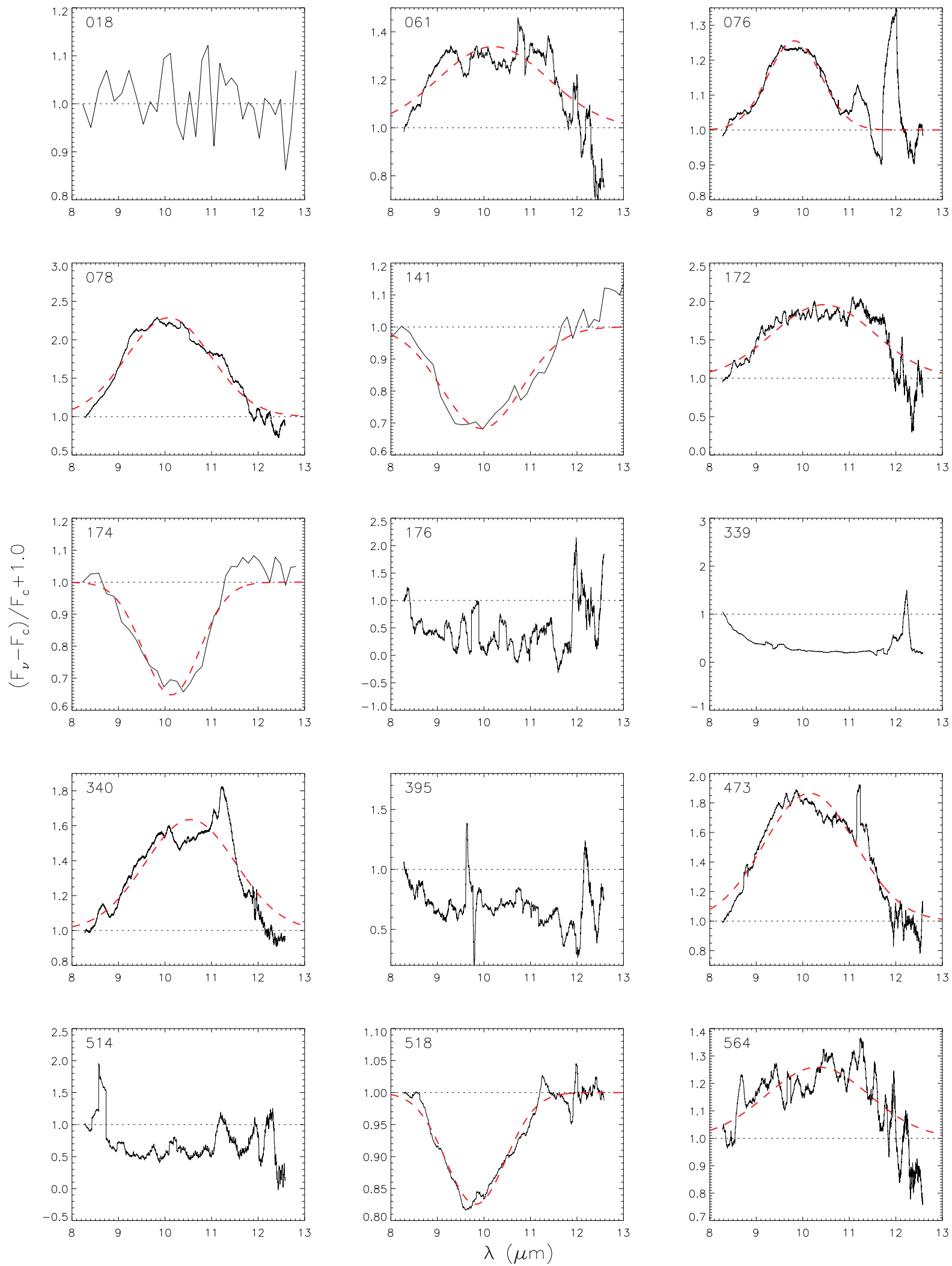

Figura 3.6: Espectros ISO SWS ou IRAS LRS dos objetos de nossa amostra. Estes espectros foram normalizados e compreendem a região espectral entre $8 \mu \mathrm{m}$ e $13 \mu \mathrm{m}$. As linhas tracejadas representam os ajustes gaussianos calculados. 
Desta definição decorre que $\alpha_{2-25}>0$ é característico de objetos mais embebidos, enquanto que $\alpha_{2-25}<0$ é típico de objetos menos embebidos. A partir dos resultados apresentado na Tabela 3.3, podemos verificar a relação entre a intensidade do perfil do silicato e a inclinação da SED. A Figura 3.7 exibe a relação entre $\alpha_{2-25}$ e a intensidade estimada pelo ajuste gaussiano dos objetos de nossa amostra. Para fins comparativos, também incluímos os resultados do trabalho de KS05.

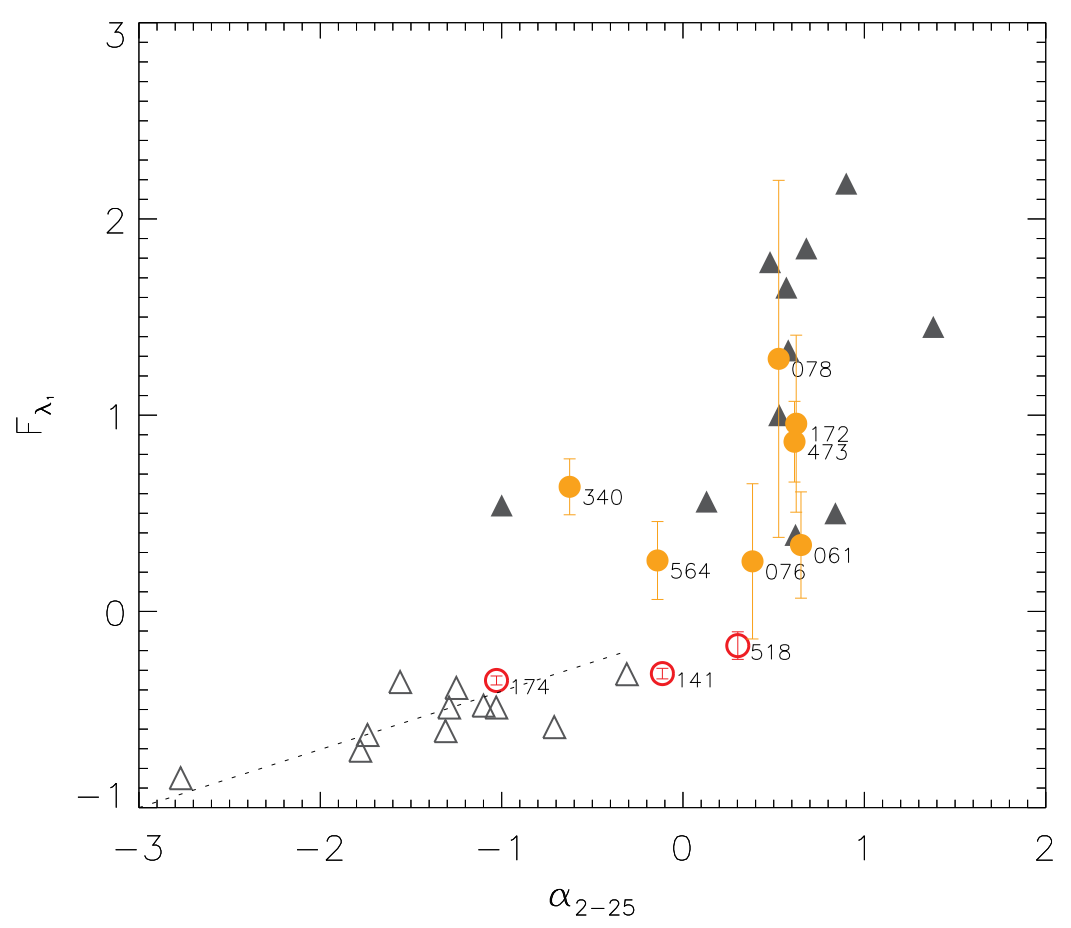

Figura 3.7: Relação entre o índice espectral IR e a intensidade do perfil de emissão/absorção do silicato. Os círculos representam os objetos de nossa amostra, enquanto que os triângulos os alvos estudados por KS05. Símbolos cheios se referem aos perfis em emissão, vazios em absorção. A linha pontilhada representa a correlação sugerida por KS05 entre a intensidade dos perfis de absorção e a extinção causada pelo meio.

Em linhas gerais, a distribuição de nossos objetos sobre o diagrama $\alpha_{2-25} \times F_{\lambda_{1}}$ segue um comportamento análogo ao observado para a amostra de KS05. Os objetos pertencentes ao Grupo 1, PDS141, PDS174 e PDS518, ocupam posições que reforçam a tendência sugerida por KS05 para os perfis em absorção. Esta correlação demonstra que a profundidade do perfil de absorção é proporcional à extinção causada pelo ambiente que ela atravessa. 
O índice espectral $\alpha_{2-25}$ é diretamente correlacionado com o efeito da extinção neste meio, sendo portanto uma grandeza útil para a quantificar os efeitos ambientais sobre o perfil do silicato. Note ainda que a intensidade do perfil de absorção de PDS518 é relativamente menor do que as outras, caracterizando possivelmente uma fase de transição entre absorção e emissão. Seu índice espectral, ao contrário dos outros dois objetos do Grupo 1, já possui um valor positivo, o que indica um status evolutivo mais avançado da estrutura circunstelar. Este resultado reforça a hipótese de que a evolução do perfil do silicato segue a evolução global do material circunstelar.

Quanto aos objetos com perfil em emissão, podemos ver que eles não apresentam dependência com a inclinação da SED. KS05 argumentam que como o perfil em emissão é produzido em um meio opticamente fino, as características espectrais intrínsecas predominam sobre as ambientais. Além disto, observamos uma grande dispersão dos valores de intensidade, o que também é compatível com a interpretação de KS05.

Um resultado interessante é a constatação de que alguns de nossos objetos ocupam a região que corresponde à transição entre os perfis de emissão e absorção no diagrama. Esta região apresentava um intervalo vazio na distribuição da amostra de KS05. Nossos resultados mostram que a transição entre os perfis observados deve ser contínua, seguindo a evolução da extinção causada pelo ambiente circunstelar. Neste ponto, novamente damos ênfase especial ao objeto PDS518, que possui perfil em absorção e índice espectral positivo.

Estudamos também a dependência entre a posição de pico (ou mínimo) e a largura do perfil de emissão (ou absorção). Esta relação pode ser observada na Figura 3.8. Podemos notar nesta figura que os objetos de nossa amostra seguem o mesmo comportamento descrito por KS05: (i) os perfis em absorção ocupam uma maior faixa em valores de FWHM e seus mínimos tendem a ocorrer em menores comprimentos de onda; e (ii) os perfis em emissão possuem uma menor dispersão em larguras e possuem posição de máximo mais deslocada para o vermelho. 


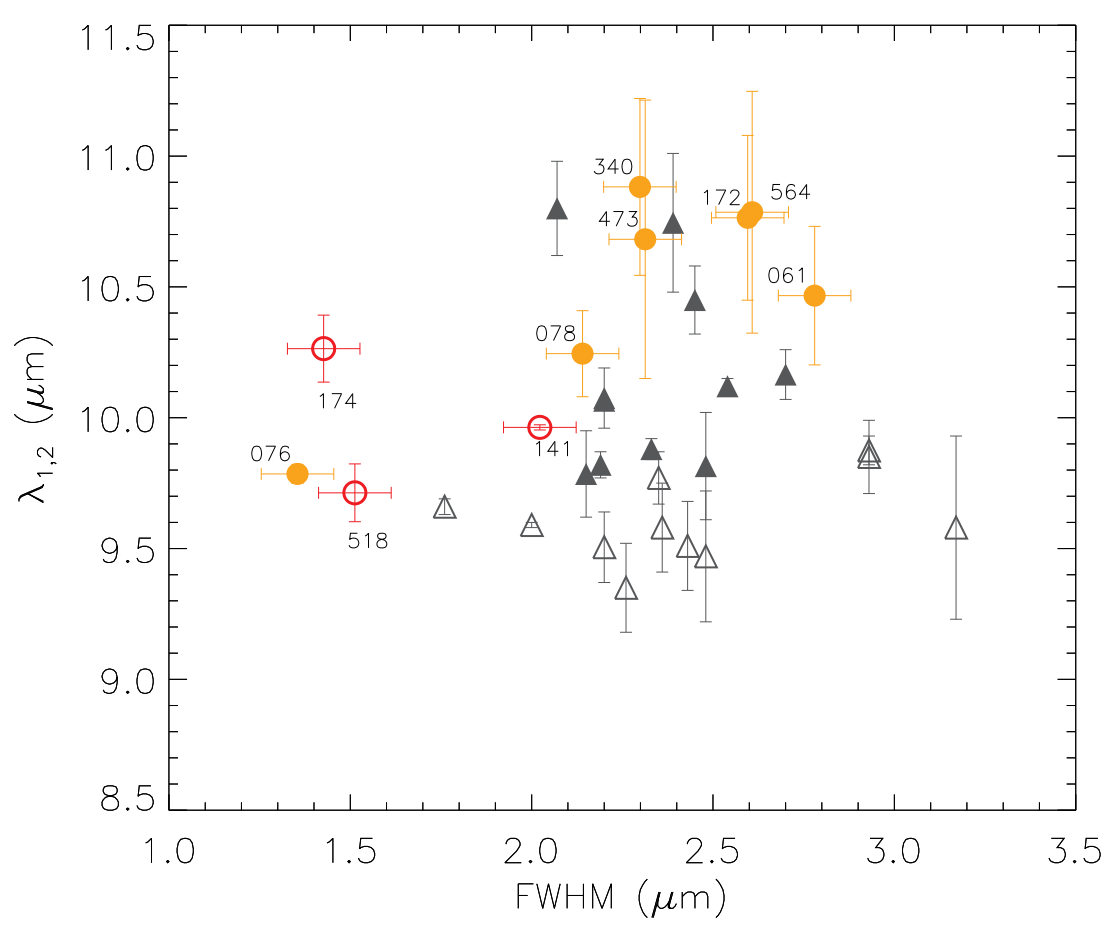

Figura 3.8: Distribuição das larguras a meia altura dos perfis de silicato em função da posição do pico de emissão (ou mínimo de absorção). Os resultados obtidos no trabalho de KS05 por meio de observações Keck também encontram-se representados na figura, para fins comparativos. O significado dos símbolos segue as definições descritas na Figura 3.7

As estrelas do PDS que apresentam absorção encontram-se no limite inferior do intervalo de larguras definido pela amostra de $\mathrm{KS} 05$, já além da região ocupada pelos perfis em emissão. Isto porque esta grandeza é bastante dependente da grande dispersão do efeito da extinção causada pelo material circunstelar destes objetos. Já no caso dos objetos com perfil em emissão, o meio circunstelar é menos espesso e portanto as características espectrais instrínsecas devem prevalecer.

O único caso que não segue este comportamento é o de PDS076. O perfil de emissão deste objeto apresenta um valor de largura abaixo da faixa ocupada pelos outros objetos com perfil em emissão. Uma possível explicação para este comportamento é a superposição entre perfis de absorção e emissão neste caso especial. Note que PDS076 encontra-se muito próximo da região de transição da Figura 3.7, o que é totalmente compatível com a hipótese de superposição. 
Por fim, analisamos a relação entre a posição do pico $\lambda_{1,2}$ e a sua intensidade, apresentada na Figura 3.9. Neste caso, apenas os perfis em emissão foram considerados. A tendência exibida neste gráfico é compatível com o crescimento dos grãos, conforme sugerido por van Boekel et al. (2005). A conversão de material amorfo em material cristalino também poderia em princípio ser a causadora do deslocamento do pico de emissão para comprimentos de onda mais longos. Todavia, neste caso esperaríamos que o deslocamento do pico não fosse seguido pelo decréscimo da intensidade.

Novamente, apenas o objeto PDS076 apresenta um comportamento diferente ao apresentado pelo resto da amostra. Sua intensidade é relativamente pequena frente aos outros objetos de mesma posição de pico. Mais uma vez, a hipótese de uma superposição entre perfis de absorção e emissão explicaria o comportamento observado.

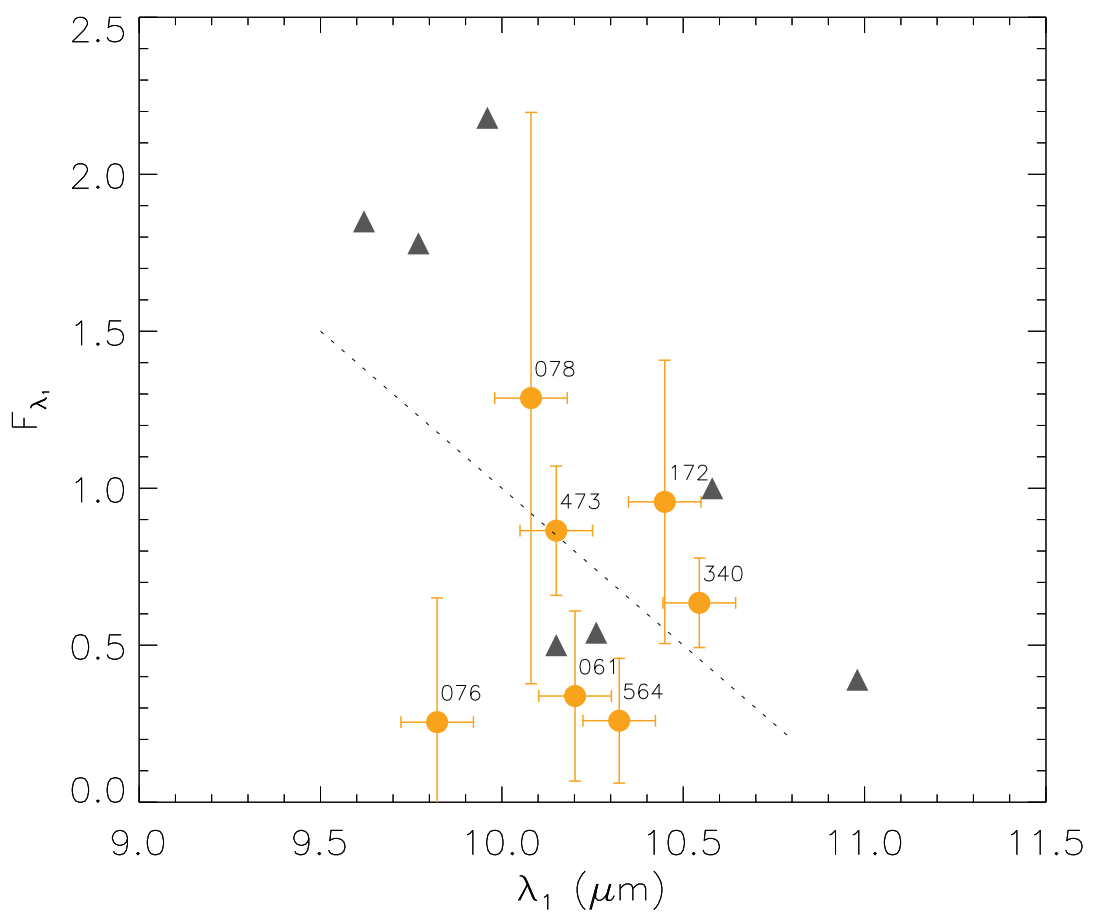

Figura 3.9: Intensidade em função da posição de pico de emissão dos objetos de nossa amostra. Os resultados obtidos por KS05 também são exibidos na figura. A linha tracejada indica a correlação apontada por KS05. Símbolos descritos na Figura 3.7 


\subsection{Composição dos grãos de poeira}

A determinação da composição das espécies de grão de poeira presentes no material circunstelar consiste em uma parte importante na caracterização deste ambiente. Trabalhos publicados por van Boekel et al. (2005) e Bouwman et al. (2008) utilizam a combinação linear da emissão das componentes mais comuns para reproduzir o perfil observado do silicato.

Em uma análise preliminar, resolvemos utilizar o código DUSTY (Ivezic e Elitzur, 1997) de transporte radiativo para estimar as abundâncias do objeto PDS061 (escolhido arbitrariamente). O DUSTY realiza os cálculos, em uma dimensão, da absorção e do espalhamento da radiação de uma fonte central que ilumina um ambiente de poeira. Este meio é caracterizado pela composição química da poeira, distribuição de tamanhos dos grãos, perfil de densidade e temperatura da camada mais interna. O código dispõe das propriedades ópticas dos tipos de grão mais comuns no meio interestelar e admite a introdução de outras espécies de poeira. As componentes de poeira utilizadas foram os silicatos "morno" e "frio" de Ossenkopf et al. (1992), silicatos e grafites de Draine e Lee (1984), carbono amorfo de Hanner (1988), e SiC de Pegourie (1988).

Desta forma, nos deparamos com o desafio do ajuste dos vários parâmetros exigidos pelo modelo. É necessária a determinação de seis frações de espécies químicas (o que na prática corresponde a cinco parâmetros livres), a temperatura da camada interna do envoltório de poeira e a profundidade óptica deste meio. Por conveniência, decidimos adotar a distribuição padrão de tamanhos de grãos oferecida pelo código, dada pela lei de potência $n(a) \propto a^{-3.5}, \operatorname{com} 0.005 \mu \mathrm{m} \leq \mathrm{a} \leq 0.25 \mu \mathrm{m}$ (Mathis et al., 1977). Como temperatura da fonte central, utilizamos o valor da temperatura efetiva determinada por Vieira et al. (2003), $T_{\text {eff }}=10072 \mathrm{~K}$. Temos portanto sete parâmetros a serem ajustados, o que em princípio não é um problema trivial.

Para a determinação sistemática destes parâmetros, decidimos implementar a técnica de algoritmos genéticos (GA - Holland 1992). Os GA representam o método ideal para lidarmos com o problema de ajustes n-dimensionais. Eles se valem de conceitos evolu- 
cionistas da biologia, que se mostram úteis para garantir a adaptação e a sobrevivência das espécies. Nesta analogia, um conjunto de soluções possíveis de nosso problema (i.e., com parâmetros dentro de intervalos de valores típicos) representa uma geração de indivíduos de uma dada população. Cada indivíduo possui suas características mapeadas por seus genes, os quais representam os parâmetros a serem ajustados em nossa analogia. Por conseguinte, a "evolução desta população" se traduz na convergência das soluções para a solução de melhor ajuste, e se dá através da aplicação do que chamamos de operadores genéticos. Eles consistem em permanência, permutação, mutação e eliminação. Em particular, as mutações garantem a varredura de todo espaço de parâmetros, evitando assim o confinamento em mínimos locais.

No nosso caso específico, permitimos que a temperatura da camada interna pudesse variar entre $1000 \mathrm{~K}$ e $2000 \mathrm{~K}$, sendo este o intervalo típico de temperaturas de sublimação da poeira. O valor da profundidade óptica pode ser estimado a partir da relação $A_{V}=$ $1.086 \times \tau_{V}$. O cálculo da extinção $A_{V}=3.1 \times E(B-V)$ nos leva a um valor de 0.7 mag. No entanto, os mapas de extinção de Dobashi et al. (2005) indicam uma extinção de 0.2 mag na direção do objeto PDS061. O valor de $A_{V}$ calculado pelo excesso de cor representa a combinação da extinção circunstelar e da interestelar, sendo portanto um limite superior para o valor de $\tau_{V}$. A estimativa obtida por meio do mapa de extinção de Dobashi et al. (2005) é baseada no método clássico de contagem de estrelas, sendo portanto uma medida da absorção interestelar. Por isto, decidimos adotar $0.4 \leq \tau_{V} \leq 0.7$ como intervalo admissível para este parâmetro.

Os parâmetros livres do modelo foram ajustados de forma a minimizar o desvio quadrático entre o modelo e o espectro ISO na região entre $8 \mu \mathrm{m}$ e $13 \mu \mathrm{m}$. Este desvio foi normalizado pelo valor do número de pontos contidos na grade de comprimentos de onda nesta faixa espectral. Um conjunto de 100 conjuntos de parâmetros foi utilizado para cada "geração", onde a cada passo as 10 melhores soluções eram mantidas e 15 mutações eram introduzidas. A Figura 3.10 exibe a evolução do desvio quadrático.

Os parâmetros determinados pelo melhor ajuste são apresentados na Tabela 3.4. O 


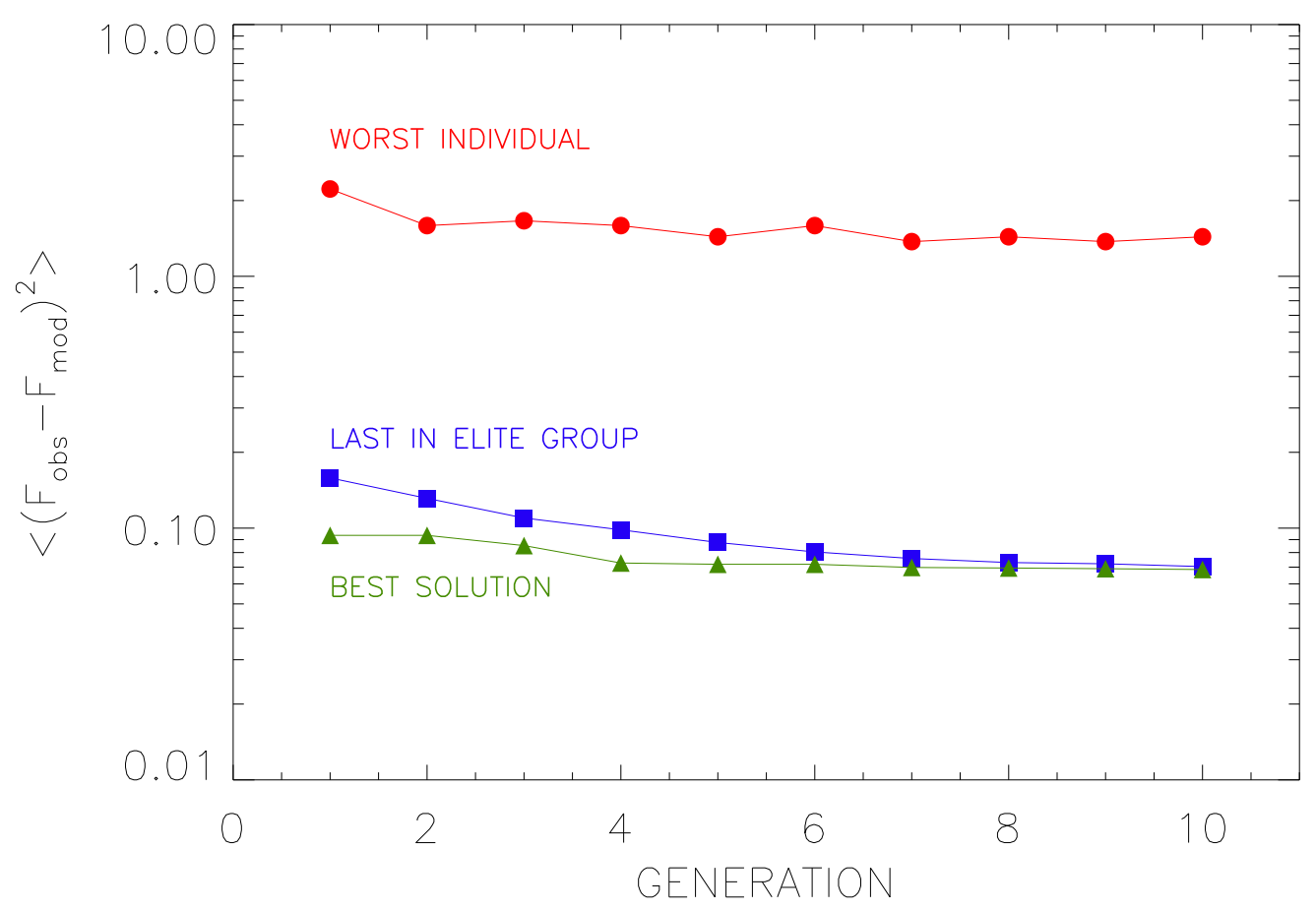

Figura 3.10: Exemplo de aplicação de algoritmos genéticos, mostrando a evolução da qualidade das soluções ao longo das várias "gerações de indivíduos". Os triângulos em verde representam a melhor solução, os quadrados azuis mostram a solução do grupo de elite com o maior desvio quadrátido e os círculos vermelhos a pior solução.

modelo correspondente pode ser encontrado na Figura 3.11, onde ele é sobreposto ao espectro normalizado e também à SED de PDS061.

Nossos resultados mostram que, se adotamos as componentes de poeira fornecidas pelo código DUSTY, o carbono amorfo possui a fração mais importante na química dos grãos de PDS061. O silicato e grafite de Draine e Lee são as duas outras componentes mais abundantes, enquanto os silicatos de Ossenkopf et al. são praticamente ausentes no seu material circuntelar.

No entanto, devemos chamar atenção ao fato de que esta determinação representa apenas um estudo preliminar da química dos objetos de nossa amostra. O código DUSTY, apesar de realizar os cálculos de trasporte radiativo sem aproximações, é capaz de lidar 

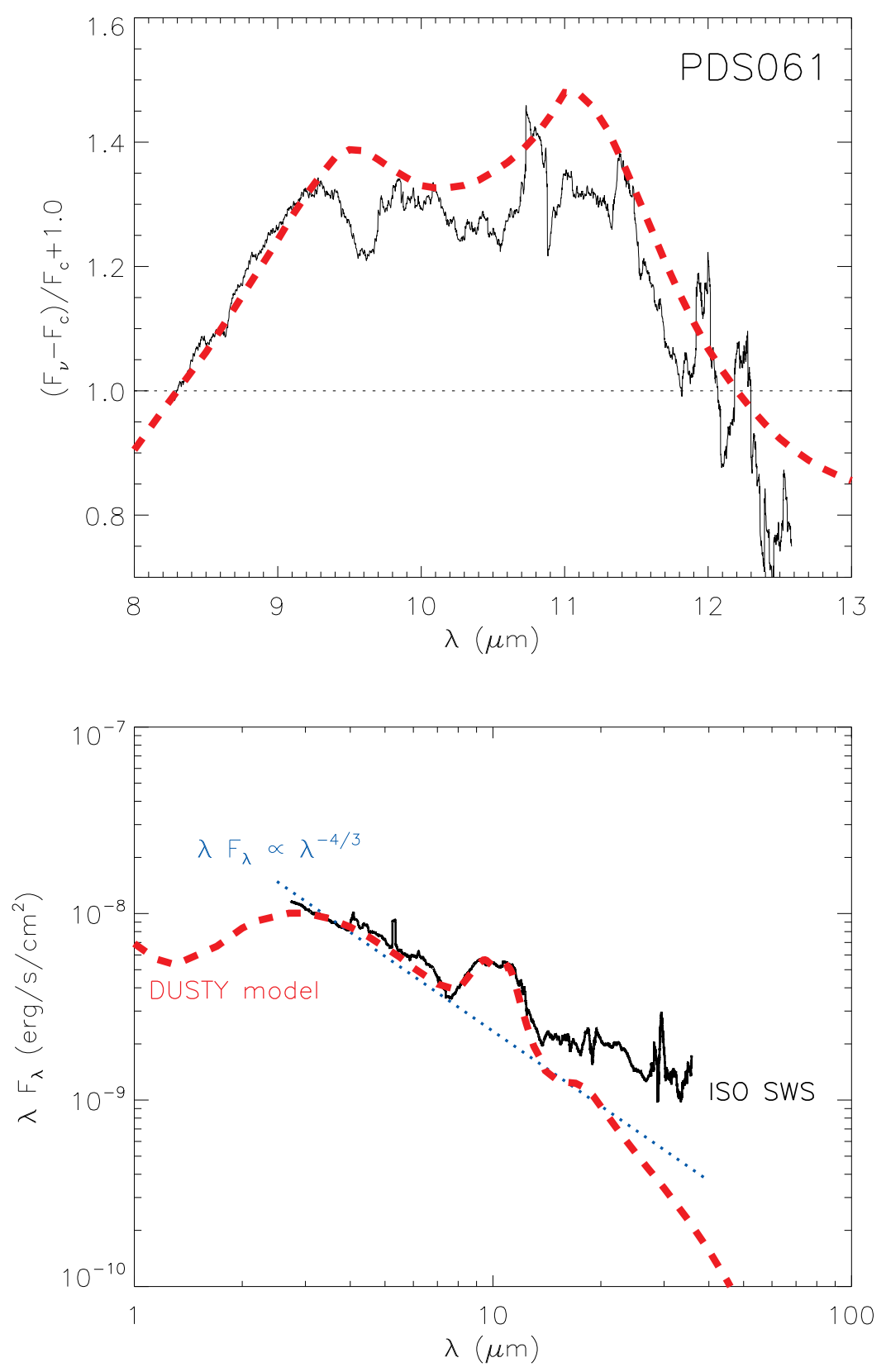

Figura 3.11: Modelo produzido pelo código DUSTY, sobreposto ao espectro de PDS061. O painel superior exibe o detalhe da região espectral ajustada, enquanto que o painel inferior mostra a distribuição de energia completa. A linha pontilhada indica a inclinação característica de um disco geometricamente fino e opticamente espesso. 
Tabela 3.4 - Parâmetros do melhor ajuste do espectro de PDS061, reproduzido pelo código DUSTY de transporte radiativo. O valor de $T_{e f f}$ foi mantido fixo, de acordo com a determinação feita por Vieira et al. (2003). A temperatura da camada mais interna do envoltório $T_{\text {in }}$ pôde variar entre $1000 \mathrm{~K}$ e $2000 \mathrm{~K}$, enquanto o intervalo de profundidades ópticas adotado foi de 0.4 a 0.7 .

\begin{tabular}{lc}
\hline \hline Parâmetro & ajuste \\
\hline$T_{\text {eff }}(\mathrm{K})$ & 10072 \\
$T_{i n}(\mathrm{~K})$ & 1131 \\
$\tau_{V}$ & 0.59 \\
Silicato "morno" de Ossenkopf et al. & $3.3 \%$ \\
Silicato "frio" de Ossenkopf et al. & $1.9 \%$ \\
Silicato de Draine \& Lee & $19.9 \%$ \\
Grafite de Draine \& Lee & $22 \%$ \\
Carbono amorfo de Hanner & $40 \%$ \\
SiC de Pègouriè & $12.9 \%$ \\
\hline \hline
\end{tabular}

somente com o caso unidimensional. No nosso caso, utilizamos a distribuição esférica de densidade, a qual não é compatível com as estruturas reais observadas. Apesar de termos reproduzido o perfil de emissão entre $8 \mu \mathrm{m}$ e $13 \mu \mathrm{m}$, podemos notar na Figura 3.11 que o formato da SED observado é distinto do modelo. O ajuste completo da distribuição de energia exige a proposição de estruturas circunstelares mais realistas.

Além disto, as componentes de poeira mais comuns utilizadas na literatura atual são as olivinas e os piroxenos, o que torna a composição padrão utilizada pelo DUSTY pouco usual. Uma análise mais completa também exigiria que a distribuição de tamanhos dos grãos fosse determinada pelo modelo.

O perfil de emissão/absorção dos silicatos é produzido na superfície dos discos circunstelares, a apenas algumas unidades astronômicas do objeto central (e.g., van Boekel et al. 2005). Podemos observar na Figura 3.11 que a inclinação da região de menores comprimentos de onda segue a lei de potência do tipo $\lambda F_{\lambda} \propto \lambda^{-4 / 3}$. Este comportamento é compatível com o presença de um disco geometricamente fino e opticamente espesso. Provavelmente, a estrutura circunstelar é mais complexa do que este modelo simplificado. Todavia, a sugestão desta lei de potência deve ser interpretada apenas como um indicativo da necessidade da presença de um disco ao redor deste objeto. 
Capítulo 4

\section{Conexão entre a massa do envoltório e o estágio evolutivo}

O excesso na emissão infravermelha (IR) de estrelas jovens sugere a presença de material circunstelar associado a estes sistemas. A energia potencial gravitacional do objeto central se converte em energia radiativa, devido à sua contração. Esta energia é absorvida e re-emitida pela poeira ao seu redor, dando origem à emissão IR observada. A luminosidade de acreção também desempenha um papel importante como fonte de energia no caso de sistemas ativos, por meio do processo viscoso de passagem do material do disco para a estrela.

Lada (1987) sugere uma classificação empírica de objetos jovens, baseada no índice espectral $\alpha_{I R}=d \log \left(\lambda F_{\lambda}\right) / d \log (\lambda)$. Este índice quantifica o excesso IR na região entre $2.2 \mu \mathrm{m}$ e $20-25 \mu \mathrm{m}$, sendo basicamente uma medida da inclinação da SED neste região. No mesmo trabalho, Lada sugere uma sequência evolutiva baseada no excesso de emissão infravermelha, onde este excesso supostamente diminui com o tempo à medida que o material circunstelar evolui. Posteriormente, André et al. (1993) incorporaram um estágio anterior a esta classificação, correspondente à fase do colapso protoestelar. As definições atuais das classes definidas por estes trabalhos são descritas a seguir:

- Classe 0 (não emite do IR próximo): estágio de colapso protoestelar, onde a SED emite aproximadamente como um corpo negro a $30 \mathrm{~K}$. A massa do envoltório é ainda muito maior do que a massa do objeto central. Com duração de apenas algumas dezenas de milhares de anos, esta fase é marcada pela presença de fluxos moleculares de estrutura bipolar; 
- Classe I $\left(\alpha_{I R}>0\right)$ : objetos mais embebidos na pré-sequência principal, que acabaram de passar pelo colapso protoestelar. Esta fase é caracterizada por distribuições de energia bastante alargadas, com pico em torno de $100 \mu \mathrm{m}$, e a massa do objeto central já é similar à massa do envoltório. Estes sistemas jovens possuem discos de acreção bem definidos e cavidades bipolares em seu envoltório (e.g., Terebey et al. 1984);

- Classe II $\left(-1.5 \leq \alpha_{I R} \leq 0\right)$ : o excesso de emissão possui pico no IR próximo, devido à presença de um disco de acreção. O envoltório circunstelar já se depositou sobre o disco ou já se dispersou completamente;

- Classe III $\left(\alpha_{I R}<-1.5\right)$ : a distribuição de energia já é bem próxima da emissão fotosférica, restando apenas um pequeno excesso no IR médio devido aos últimos resquícios do material circunstelar.

Originalmente Lada (1987) havia estabelecido o valor de $\alpha_{I R}=-2$ como o limite entre as Classes II e III, porém este valor foi reajustado para $\alpha_{I R}=-1.5$ por André e Montmerle (1994). Uma representação esquemática dos estágios iniciais da evolução de uma estrela é exibida na Figura 4.1.

Uma das maneiras mais diretas de estudarmos a evolução do material circunstelar de estrelas jovens é através do diagrama da massa do envoltório em função da luminosidade bolométrica (André et al., 2000). Esta ferramenta é particularmente interessante para o caso dos objetos de Classe 0 e Classe I, onde o material circunstelar se encontra predominantemente contido no envoltório circunstelar (i.e., ainda não se sedimentou sobre o disco).

A Figura 4.2 apresenta a distribuição de uma amostra de André et al. (2008) sobre este diagrama. Esta amostra contém objetos de Classe 0 e Classe I situados em Ophiucus, Perseus e Orion. As retas que cruzam o diagrama representam a separação conceitual entre os objetos de Classe 0 e Classe I. A linha tracejada é dada por $M_{e n v} \propto L_{b o l}$ (André e Montmerle, 1994), enquanto que a linha pontilhada segue a relação $M_{e n v} \propto L_{b o l}^{0.6}$. Esta última relação é sugerida pelo cenário de acreção adotado nas trajetórias evolutivas, as quais são calculadas supondo-se que as protoestrelas se formam a partir de condensações de tamanho e massa finitos. 

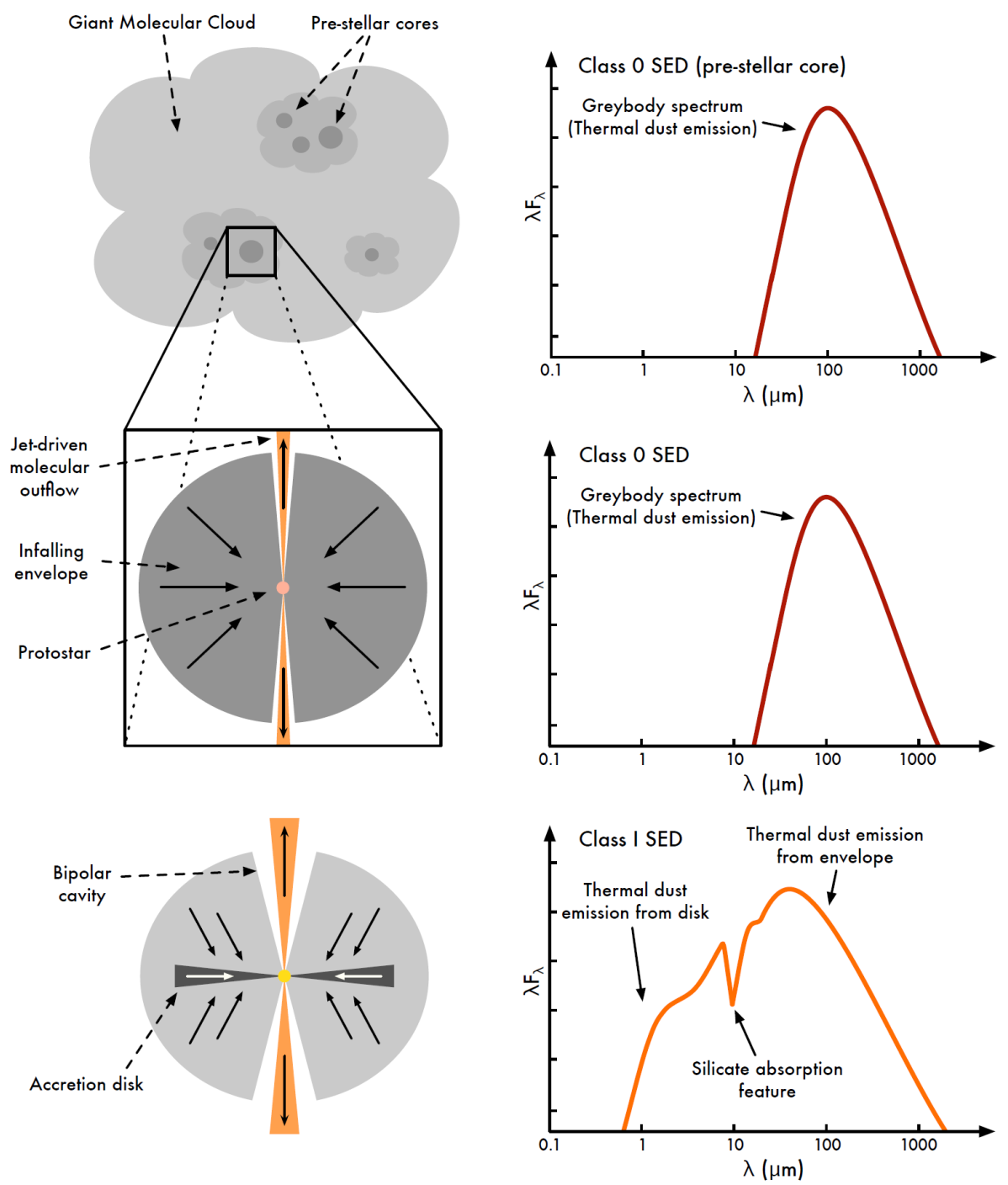

Figura 4.1: Evolução de um objeto estelar jovem. No topo à esquerda temos a representação esquemática de condensações formadas no interior de uma nuvem molecular gigante. No centro à esquerda temos o início do colapso de uma única condensação (Classe 0). O painel inferior exibe o estágio posterior a este colapso, onde o material se deposita sob a forma de um disco devido à conservação do momento angular, que por sua vez alimenta o objeto central. Os paineis à direita representam as SEDs relativas à cada um dos estágios evolutivos apresentados. Figura adaptada de Robitaille (2008). 
A luminosidade bolométrica é dada por $L_{b o l}=\left(G M_{*} \dot{M}_{a c c} / R_{*}\right)+L_{*}$, sendo $L_{*}$ a luminosidade da linha de nascimento na PMS. Tanto $M_{e n v}$ como $\dot{M}_{a c c}=M_{e n v} / \Delta t\left(\Delta t=10^{5}\right.$ anos), decrescem exponencialmente com o tempo neste cenário proposto (Bontemps et al., 1996). As massas estelares finais são indicadas sobre o eixo de $L_{b o l}$.

Outra característica do diagrama $L_{b o l} \times M_{e n v}$ é que as massas de envoltório são normalizadas para a massa contida em um diâmetro de 6000 AU. Este valor representa o comprimento típico da escala de fragmentação de regiões de formação estelar próximas (Peretto et al., 2007).

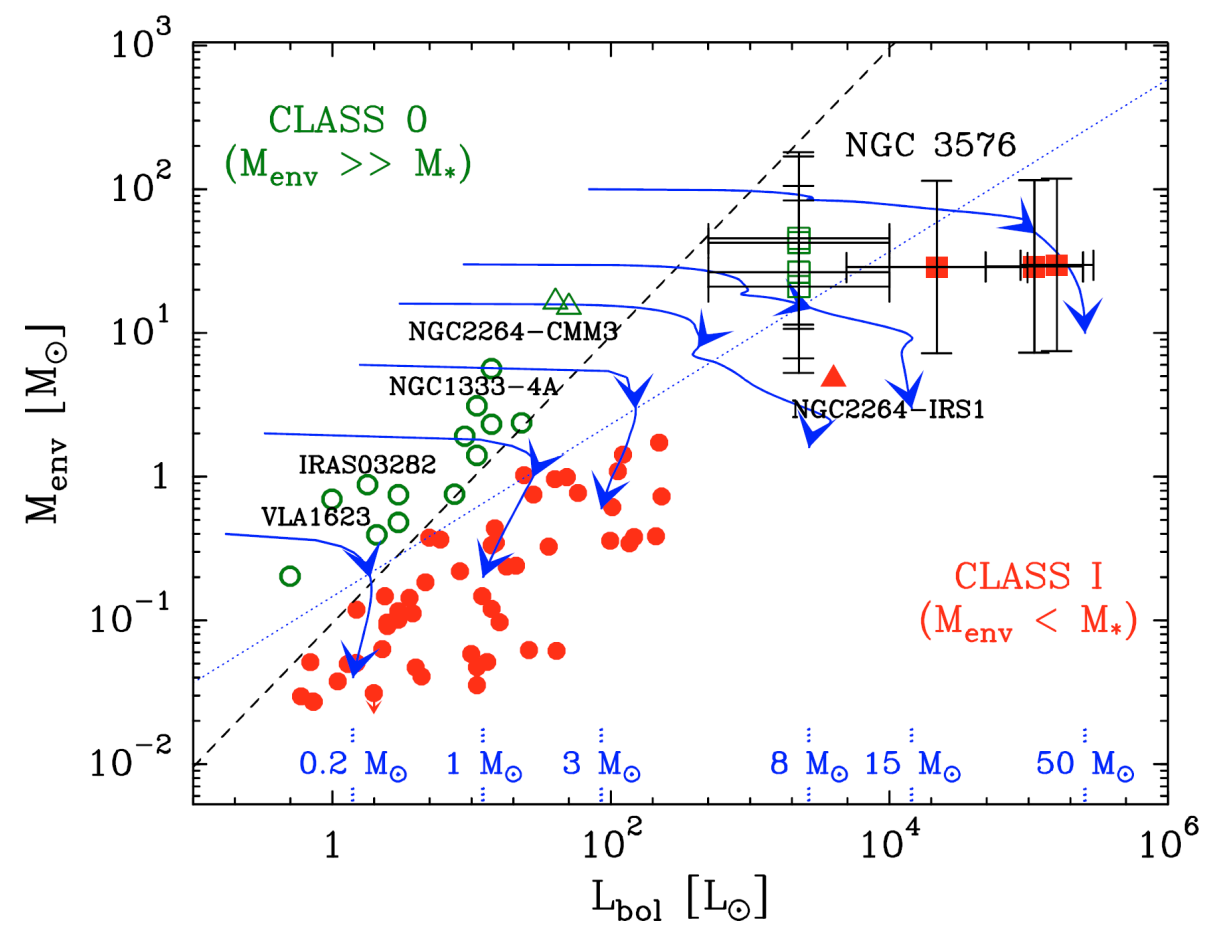

Figura 4.2: Diagrama $L_{b o l} \times M_{e n v}$ para amostra de Classe 0 (símbolos abertos) e Classe I (símbolos fechados) em Ophiuchus, Perseus e Orion (André et al., 2000). Também encontramse no diagrama protoestrelas em NGC 3576 (quadrados) e em NGC 2264 (triângulos Peretto et al. 2007; Maury et al. 2009). São superpostas as trajetórias evolutivas de protoestrelas, para várias massas estelares finais (indicadas sobre o eixo $L_{b o l}$ ). Em cada uma das trajetórias, as flechas indicam as posições onde 50\% e 90\% da massa final do objeto estelar já foi acretada. Figura adaptada de André et al. (2008). 
Desta forma, vemos que o diagrama indica não apenas o estágio evolutivo de estrelas jovens, mas também a sua massa final, sendo portanto uma ferramenta bastante útil no estudo destes objetos.

A massa do envoltório pode ser estimada a partir dos fluxos milimétricos/submilimétricos, utilizando-se a relação (e.g., Minier et al. 2005; André et al. 2008):

$$
M_{e n v}=\frac{F_{\lambda} D^{2}}{R_{d} \kappa_{\lambda} B_{\lambda}\left(T_{d}\right)}
$$

Nesta equação, $F_{\lambda}$ representa o fluxo medido na região do $\mathrm{mm} / \mathrm{sub}-\mathrm{mm}, D$ a distância do objeto, $R_{d}$ a razão entre a massa de gás e a massa da poeira (tipicamente na proporção de $100: 1), \kappa_{\lambda}$ a opacidade e $B_{\lambda}\left(T_{d}\right)$ a emissão de corpo negro da poeira a temperatura $T_{d}$.

Esta aproximação pressupõe que o meio seja opticamente fino, sendo normalmente adotada uma opacidade sob a forma de lei de potência $\kappa_{\lambda}=\kappa_{0}\left(\lambda_{0} / \lambda\right)^{\beta}$. Os valores de $\beta$ encontram-se tipicamente entre 1 e 2.

Apesar das hipóteses adotadas serem válidas nesta faixa de comprimentos de onda, não conhecemos a priori os valores da temperatura da poeira, do expoente $\beta$ e a razão de massa gás-poeira. Valores tipicamente adotados na literatura são $T=20 \mathrm{~K}, \beta=2$ e $R_{d}=100$, o que não é necessariamente válido para todos os casos.

Hill et al. (2009) estimam o valor da temperatura utilizando o método baiesiano de inferência. Neste trabalho, os autores adotam uma componente morna e uma componente fria para o ajuste da SED de uma amostra de protoestrelas, dada por

$$
F_{\nu}=\left[\pi B_{\nu}\left(T_{\text {hot }}\right) R_{\text {hot }}^{2}+B_{\nu}\left(T_{\text {cold }}\right) M_{\text {cold }} \kappa(\nu)\right] / D^{2}
$$

A temperatura da componente fria assume valores entre $2.73 \mathrm{~K}$ e $100 \mathrm{~K}$, abrangendo portanto um intervalo considerável. Para se ter uma ideia do quanto a estimativa da massa do envoltório é sensível a este valor, basta fazermos uma simples estimativa. Se considerarmos um valor típico de $\lambda=1 \mathrm{~mm}$ e os limites do intervalo de temperaturas determinadas por Hill et al., temos que $B_{1 \mathrm{~mm}}(100 \mathrm{~K}) / \mathrm{B}_{1 \mathrm{~mm}}(2.73 \mathrm{~K}) \sim 10^{3}$, o que significa que a massa 
do envoltório pode ser sub/super-estimado em até três ordens de grandeza.

Neste capítulo, estudaremos o status evolutivo de uma sub-amostra das estrelas do tipo HAeBe do PDS. Também incluiremos na análise quatro candidatas a estrelas massivas, estudadas durante o estágio de doutorado realizado no Centro de Energia Atômica de Saclay (França), sob a supervisão do pesquisador Vincent Minier.

Nas próximas seções, serão descritos: (i) a amostra selecionada e seus dados disponíveis; (ii) os métodos de determinação de massa de envoltório e luminosidade bolométrica; (iii) e finalmente os resultados obtidos através da análise do diagrama $L_{b o l} \times M_{e n v}$.

\subsection{Objetos selecionados}

\subsubsection{Sub-amostra PDS com dados Planck}

Para realizarmos o estudo do status evolutivo no diagrama $L_{b o l} \times M_{e n v}$, selecionamos todas as estrelas HAeBe do PDS com observações disponíveis na região do mm/sub-mm. Foram encontrados dados de onze destes objetos no catálogo Planck de fontes compactas (Planck Early Release Compact Source Catalogue), sendo dez entre elas pertencentes ao Grupo 1 do PDS (i.e, as com maiores excessos no IR) e uma do Grupo 2 (PDS340). A Tabela 4.1 exibe os dados fotométricos destes objetos. Notemos ainda que estão incluídas nesta lista três estrelas que foram apontadas por Vieira et al. (2011) como possíveis objetos pós-AGB: PDS174, PDS204 e PDS290 (veja Capítulo 2). Apesar disto, decidimos ainda assim manter estes objetos na presente análise, dado que apenas a obtenção de novos dados irá confirmar sua real natureza.

A missão Planck é de responsabilidade da Agência Espacial Europeia (ESA), com importantes contribuições da NASA. Ela representa a terceira geração de telescópios espaciais para observar a radiação cósmica de fundo, tendo como predecessores os satélites COBE (Cosmic Background Explorer) e WMAP (Wilkinson Microwave Anisotropy). O satélite Planck foi lançado em Maio de 2009, tendo sua "primeira luz" em Agosto do mesmo ano. Suas observações abrangem os comprimentos de onda entre $350 \mu \mathrm{m}$ e $1 \mathrm{~cm}$, com resoluções 
Tabela 4.1 - Dados da missão Planck disponíveis para sub-amostra de estrelas HAeBe do PDS.

\begin{tabular}{cccccccc}
\hline \hline PDS & Planck & \multicolumn{7}{c}{ Fluxo (Jy) } \\
& & $350 \mu \mathrm{m}$ & $550 \mu \mathrm{m}$ & $850 \mu \mathrm{m}$ & $1.4 \mathrm{~mm}$ & $2.1 \mathrm{~mm}$ & $3 \mathrm{~mm}$ \\
\hline 18 & G215.87-17.49 & 105.165 & - & - & - & 0.863 & - \\
174 & G203.50-24.72 & 371.146 & 134.773 & - & - & 2.147 & - \\
204 & G184.87-01.73 & 91.384 & 31.011 & 9.267 & 2.57 & 0.59 & - \\
207 & G181.46+04.60 & 53.316 & 22.666 & 5.724 & 1.526 & - & - \\
216 & G196.94+00.61 & 60.639 & - & - & - & - & - \\
241 & G218.74+01.85 & 120.81 & 35.817 & 9.524 & 2.51 & 0.952 & 0.757 \\
249 & G239.93-05.04 & - & 22.461 & 6.563 & 2.059 & - & - \\
290 & G274.69-01.48 & 189.366 & 68.209 & 18.115 & 5.376 & - & 1.854 \\
340 & G026.80+03.53 & - & - & 1.479 & - & - & - \\
518 & G039.08+05.86 & 469.089 & - & - & - & - & - \\
530 & G296.37-08.32 & - & - & - & 1.481 & - & - \\
\hline \hline
\end{tabular}

espaciais que vão de $5^{\prime}$ a $33^{\prime}$ respectivamente.

O catálogo Planck de fontes compactas lista todos os objetos com detecção altamente confiável, observados na primeira varredura de todo o céu efetuada. A missão pretende cobrir todo o céu a cada 6 meses, observando tanto objetos Galácticos como extra-galácticos. A primeira edição deste catálogo já conta com $90 \%$ de cobertura de todo o céu, com uma precisão fotométrica de $30 \%$.

Para complementarmos estes dados, utilizamos também as fotometrias nas bandas visíveis realizadas pelo PDS (Vieira et al., 2003), dados 2MASS (Sartori et al., 2010), WISE ( Wide-field Infrared Survey Explorer - Wright et al. 2010), IRAS e AKARI (em sua maioria listados em Vieira et al. 2011).

\subsubsection{Candidatas a protoestrelas massivas}

A formação e evolução de estrelas massivas $\left(M>8 \mathrm{M}_{\odot}\right)$ desempenham um papel importante em muitos processos astrofísicos. Estes objetos são responsáveis pela produção de elementos pesados no final de suas vidas com explosões em supernovas, além de dominar a injeção de energia no meio interestelar através de ventos, radiação ionizante e das próprias supernovas. Estes mecanismos influenciam diretamente a história de formação estelar do ambiente ao redor destas estrelas, como também a própria estrutura da nossa Galáxia. 
No entanto, a origem destes objetos massivos ainda não é bem compreendida. O cenário de formação destas estrelas, ao contrário de seus análogos menos massivos, ainda gera um grande debate na literatura atual. As propostas incluem o modelo de acreção (Yorke e Sonnhalter 2002; Krumholz et al. 2007), similar ao das estrelas de menor massa, e o cenário de coalescência de objetos menos massivos.

Um dos motivos para ainda não haver resposta para esta questão fundamental são as dificuldades na observação desta classe de objetos. São poucos os exemplos conhecidos, pois além do fato da função de massa inicial privilegiar o formação de estrelas menos massivas, os sítios de formação de estrelas massivas normalmente encontram-se muito distantes do Sol. Além disto, as estrelas de alta massa são normalmente encontradas em ambientes com alta densidade estelar, o que dificulta ainda mais a sua detecção (Motte et al., 2010).

Outro inconveniente no estudo destes objetos é a alta extinção presente em suas regiões de formação. Estudos de McKee e Tan (2003) indicam que os sítios de formação de estrelas massivas possuem tipicamente uma extinção visual de 200 mag, o que impossibilita não só as observações nos comprimentos de onda ópticos como também no IR próximo.

Portanto, a detecção de novos exemplos de estrelas massivas em seus estágios iniciais se torna uma atividade de extrema importância. O estudo desenvolvido neste capítulo analisa as propriedades de quatro candidatas a objetos jovens massivos, de modo a fornecer novas pistas para que possamos melhor compreendê-los.

A primeira destas candidatas é o objeto G31.41+0.31 (18:47:34.33 -01:12:46.50 J2000), um núcleo quente a $7.9 \mathrm{kpc}$, associado a emissão maser de água e a uma região HII ultracompacta. Cesaroni et al. (1994) apresentaram evidências preliminares da existência de um disco massivo em rotação neste objeto, com orientação perpendicular a jatos bipolares observados em ${ }^{13} \mathrm{CO}$. A presença desta estrutura foi mais tarde confirmada por Beltrán et al. (2004), através de observações no contínuo em $1.4 \mathrm{~mm}$ e na linha de emissão de $\mathrm{CH}_{3} \mathrm{CN}(12-11)$ (Figura 4.3). Estes autores interpretaram que suas observações revelavam um toróide com eixo de rotação perpendicular ao plano do céu, velocidade de 


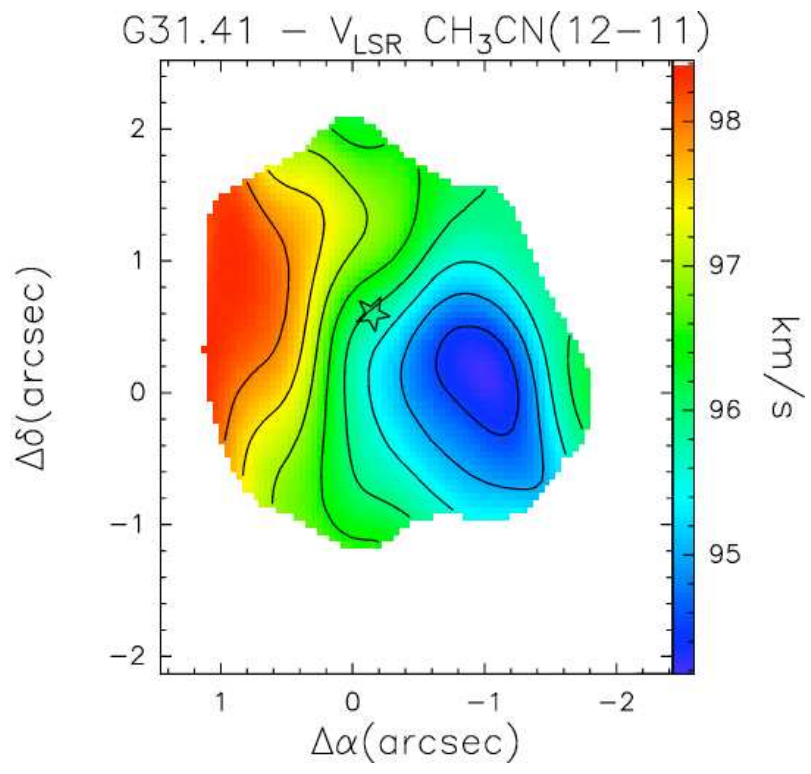

Figura 4.3: Mapa de velocidades em $\mathrm{CH}_{3} \mathrm{CN}$ obtido por Beltrán et al. (2004).

rotação de $1.7 \mathrm{~km} / \mathrm{s}$ e raios interno e externo de 1340 AU e 13400 AU, respectivamente. No entanto, através de imagens VISIR 1 (VLT - Figuras 4.4(a) e 4.4(b) de alta resolução espacial no IR médio, Minier et al. propõem que $R_{\text {in }}=6000 \mathrm{AU}$ e $R_{\text {out }}=22000 \mathrm{AU}$, com uma velocidade de rotação máxima de $2 \mathrm{~km} / \mathrm{s}$. Além disto, as imagens VISIR sugerem uma estrutura fragmentada, a qual poderia ser interpretada como a fragmentação de um disco protoestelar massivo (e.g., Kratter e Matzner 2006) ou como um proto-aglomerado de objetos jovens.

Como dispomos desta imagens VISIR, pudemos medir o fluxo nestas duas bandas infravermelhas. Desta forma, determinamos um fluxo de $0.3 \mathrm{Jy}$ em $8.59 \mu \mathrm{m}$ e de $5.2 \mathrm{Jy}$ em $18.72 \mu \mathrm{m}$. Além destas medidas, Cesaroni et al. (1994) fornecem a medida de 0.73 Jy em $2.7 \mathrm{~mm}$ e Beltrán et al. (2005) indicam os fluxos de 4.4 Jy em $1.4 \mathrm{~mm}$ e $0.67 \mathrm{Jy}$ em $3.3 \mathrm{~mm}$.

As outras três candidatas estão localizadas na região de Vela, a $(700 \pm 200)$ pc de distância. Entre elas, temos duas fontes em RCW36 (08:59:00.9 -43:44:10 J2000) e o objeto G265.3+1.43 (08:59:53.57 -43:52:17.88 J2000). Os três objetos dispõem de observações BLAST (Balloon-borne Large Aperture Sub-millimeter Telescope-Pascale et al.

\footnotetext{
${ }^{1}$ Para maiores detalhes sobre o instrumento VISIR, veja o Capítulo 5
} 
2008) em $250 \mu \mathrm{m}, 350 \mu \mathrm{m}$ e $500 \mu \mathrm{m}$, a partir da qual determinamos sua fotometria. As Figuras 4.4(c) e 4.4(d) exibem as imagens em $250 \mu \mathrm{m}$ destes objetos.

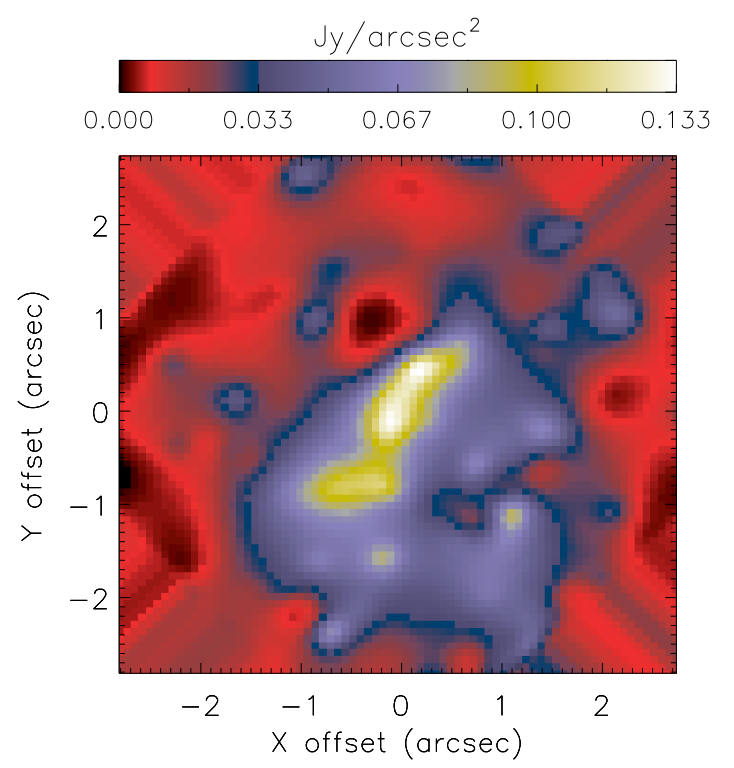

(a) G31.41+0.31 na banda PAH1 $(8.59 \mu \mathrm{m})$.

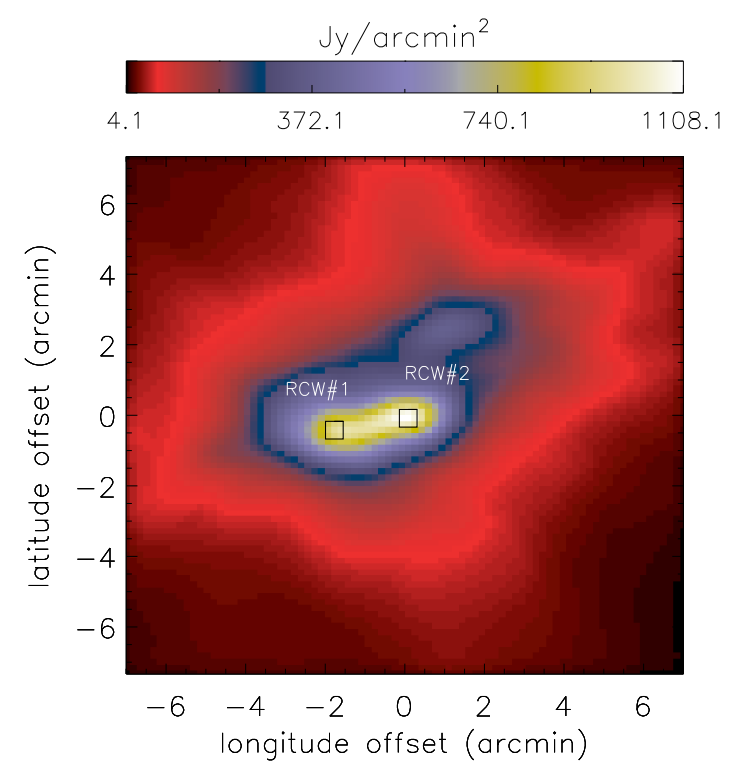

(c) Imagem BLAST em $250 \mu \mathrm{m}$ da região RCW36 .

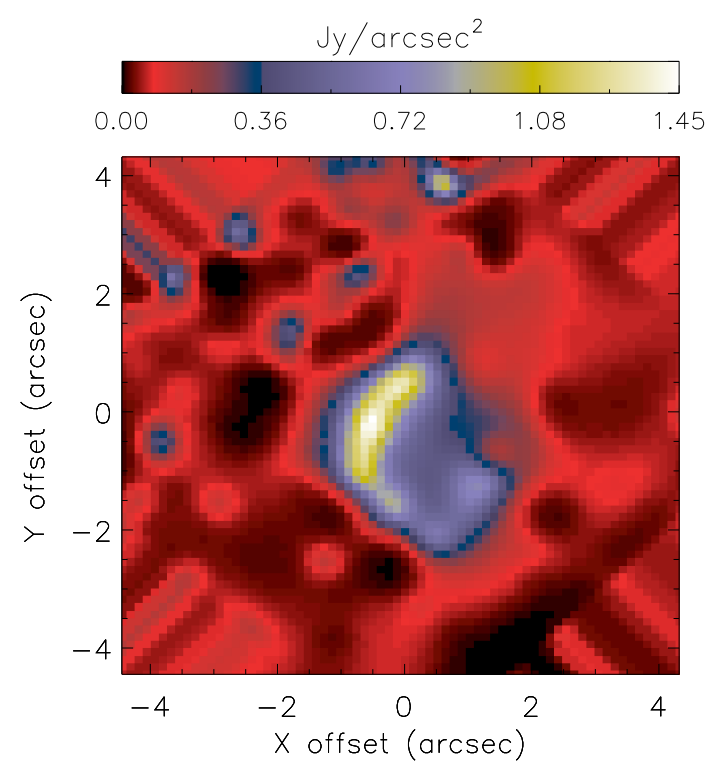

(b) G31.41+0.31 na banda Q2 $(18.72 \mu \mathrm{m})$.

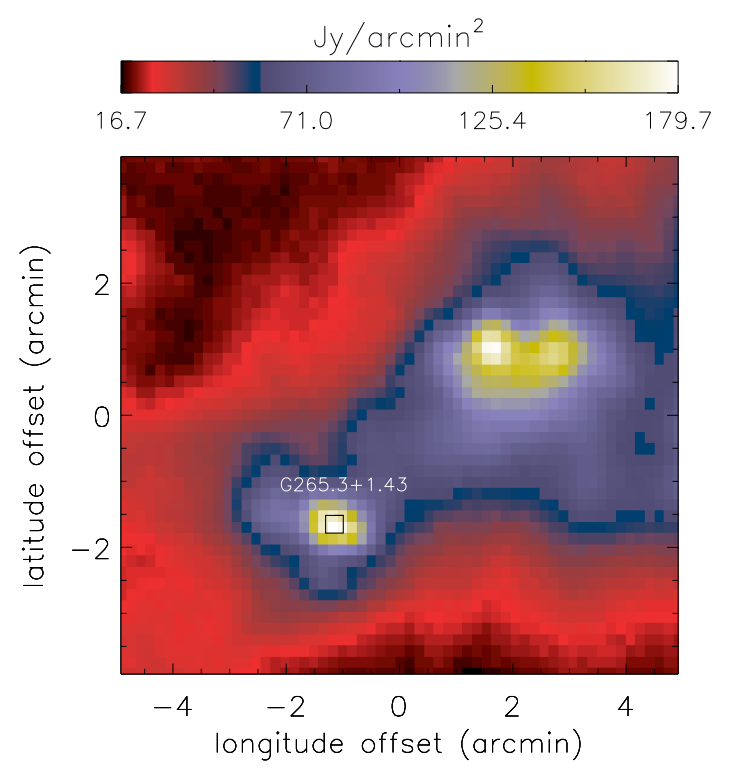

(d) Imagem BLAST em $250 \mu \mathrm{m}$ de G265.3+1.43.

Figura 4.4: Imagens de G31.41+0.31, RCW36 e G265.3+1.43. 
As fontes em RCW36 também possuem dados fotométricos dos catálogos 2MASS e MSX, medidas obtidas por Verma et al. (1993) em 58 e $150 \mu \mathrm{m}$ com o telescópio TIFR (Tata Institute of Fundamental Research) e fluxos em $1.2 \mathrm{~mm}$ de observações SIMBA (SEST IMaging Bolometer Array - Hill et al. 2005). Já para o caso de G265.3+1.43, além dos fluxos BLAST utilizamos os limites de detecção 2MASS e MSX como limites superiores de seu fluxo nestas bandas. Todas as medidas fotométricas aqui apresentadas são listadas na Tabela 4.2 .

Tabela 4.2 - Fluxos disponíveis para RCW36\#1, RCW36\#2 e G265.3+1.43.

\begin{tabular}{llccc}
\hline \hline & banda & RCW36\#1 & RCW36\#2 & G265.3+1.43 \\
\hline \multirow{2}{*}{ 2MASS (mag) } & $J$ & - & 11.8 & $>17.1$ \\
& $K_{S}$ & - & 10.4 & $>16.4$ \\
& $8.28 \mu \mathrm{m}$ & 49.6 & 68.4 & $<0.07$ \\
\hline \multirow{2}{*}{ MSX (Jy) } & $12.13 \mu \mathrm{m}$ & 76.1 & 133.1 & $<0.1$ \\
& $14.65 \mu \mathrm{m}$ & 48.7 & 98.7 & $<0.08$ \\
& $21.34 \mu \mathrm{m}$ & 239.1 & 478.2 & $<0.79$ \\
\hline \multirow{2}{*}{ TIFR (Jy) } & $58 \mu \mathrm{m}$ & 8420 & 7920 & - \\
& $150 \mu \mathrm{m}$ & 7380 & 6390 & - \\
\hline \multirow{5}{*}{ IRAS (Jy) } & $12 \mu \mathrm{m}$ & - & 66.8 & - \\
& $25 \mu \mathrm{m}$ & - & 582 & - \\
& $60 \mu \mathrm{m}$ & - & 8330 & - \\
\hline \multirow{2}{*}{ BLAST (Jy) } & $100 \mu \mathrm{m}$ & - & 15800 & - \\
\hline SIMBA (Jy) & $250 \mu \mathrm{m}$ & 1190 & 2280 & 174 \\
& $350 \mu \mathrm{m}$ & 370 & 858 & 99.5 \\
\hline \hline
\end{tabular}

\subsection{Metodologia}

Para que possamos analisar o status evolutivo dos objetos de nossa amostra, é necessário primeiramente determinar seus valores de $L_{b o l}$ e $M_{\text {env }}$. Neste trabalho, utilizamos dois métodos para a estimativa destas grandezas: (i) o ajuste da SED através do modelo disponibilizado por Robitaille et al. (2007 - ROB07 daqui em diante); (ii) a aproximação de um corpo cinza para os fluxos de maiores comprimentos de onda.

Desta forma, pudemos estabelecer uma base de comparação entre os resultados obtidos 
por ambos os métodos, analisando as vantagens e desvantagens na utilização de cada um deles. O modelo de SED de ROB07 é descrito na Seção 4.2.1, enquanto que a nossa aproximação de corpo cinza é apresentada na Seção 4.2.2.

\subsubsection{Modelo de Robitaille et al. (2007)}

O desenvolvimento de modelos de SED é especialmente interessante quando as observações não dispõem de informação espacial. Este é o caso da grande maioria dos objetos estelares jovens (YSOs) conhecidos até o momento.

Com o intuito de interpretar de forma consistente o volume crescente de dados disponíveis, ROB07 desenvolveram uma grade de modelos de transporte radiativo em objetos jovens axissimétricos. Estes modelos cobrem a extensa faixa de massas estelares entre $0.1 \mathrm{M}_{\odot} \mathrm{e}$ $50 \mathrm{M}_{\odot}$, e estágios evolutivos que vão desde o colapso primordial do envoltório até estágios avançados onde há apenas a presença do disco.

A distribuição de energia foi calculada para 20 mil conjuntos de parâmetros, cada um com 10 ângulos de visada, perfazendo um total de 200 mil soluções.

O modelo consiste no cálculo de transporte radiativo em um sistema composto por um disco e um envoltório com cavidades bipolares (Whitney et al., 2003). A estrutura do disco segue a distribuição de densidade padrão de discos de acreção alargados, dada por (Shakura e Sunvaev 1973; Bjorkman 1997; Hartmann 1998):

$$
\rho_{\text {disk }}=\rho_{0}\left[1-\sqrt{\frac{R_{*}}{\varpi}}\right]\left(\frac{R_{*}}{\varpi}\right)^{\alpha} \exp \left[-\frac{1}{2}\left(\frac{z}{h(\varpi)}\right)^{2}\right] .
$$

Na expressão, $R_{*}$ representa o raio estelar, $\varpi=\sqrt{x^{2}+y^{2}}$ a coordenada cilíndrica radial e $z$ a altura com relação ao plano central do disco. A escala de altura segue a lei de potência $h=h_{0}\left(\varpi / R_{*}\right)^{\beta}$.

A geometria proposta para o envoltório é compatível com uma estrutura em rotação, dado que esta componente provavelmente também possui momento angular. Além disto, 
cavidades bipolares foram incluídas nesta estrutura, de forma a torná-la compatível com os resultados de outros trabalhos na literatura (Padgett et al. 1999; Reipurth et al. 2000). A sua distribuição de densidade possui a seguinte expressão:

$$
\rho_{e n v}=\frac{\dot{M}_{e n v}}{4 \pi}\left(\frac{G M_{*}}{R_{c}^{3}}\right)^{-1 / 2}\left(\frac{r}{R_{c}}\right)^{-3 / 2}\left(1+\frac{\mu}{\mu_{0}}\right)^{-1 / 2}\left(\frac{\mu}{\mu_{0}}+\frac{2 \mu_{0}^{2} R_{c}}{r}\right)^{-1} .
$$

Nesta equação, $\dot{M}_{e n v}$ representa a taxa de acreção do envoltório, $R_{c}$ o raio centrífugo, $\mu=\cos (\theta)$ e $\mu_{0}=\lim _{r \rightarrow \infty} \mu(r)$. O formato da cavidade é dado por:

$$
\mu_{0}^{3}+\mu_{0}\left(r / R_{c}-1\right)-\mu\left(r / R_{c}\right)=0
$$

Além destas geometrias, o modelo também inclui a energia de acreção ao sistema, segundo a prescrição de discos- $\alpha$ (Shakura e Sunyaev 1973; Bjorkman 1997; Hartmann 1998). As rotinas de transporte radiativo utilizadas são aquelas desenvolvidas por Bjorkman e Wood (2001), implementadas em um sistema tridimensional de coordenadas esféricas. A Figura 4.5 exibe um exemplo dos resultados obtidos por ROB07.
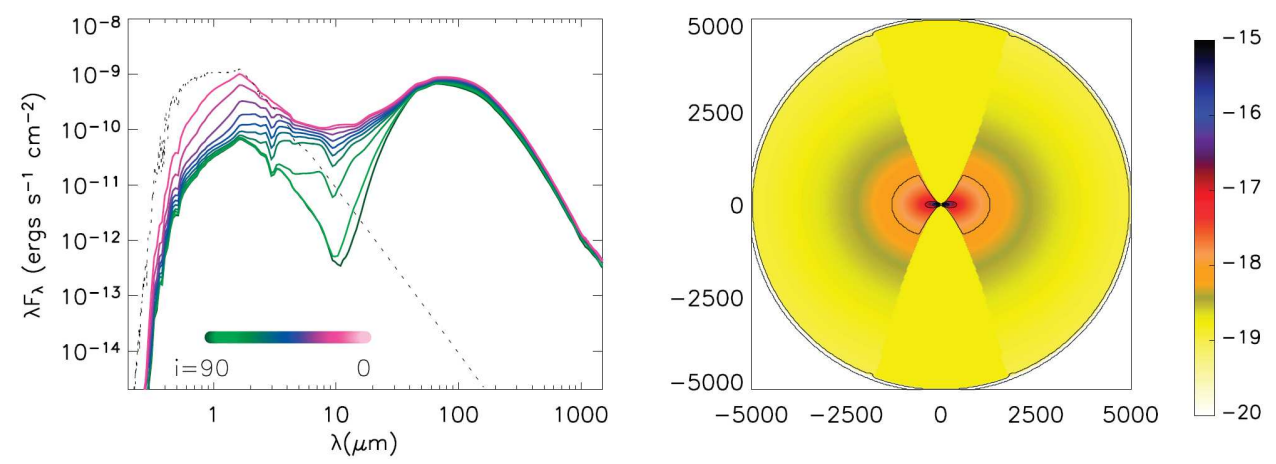

Figura 4.5: Modelo de protoestrela Classe I com geometria utilizada por ROB07. À esquerda, temos a SED resultante, para vários ângulo de inclinação, enquanto que à direita temos a distribuição de densidade do sistema. A densidade é expressa em unidades $\mathrm{de} \mathrm{g} / \mathrm{cm}^{3}$ (escala logarítmica), e os eixos das imagens são dados em unidades astronômicas. Figura adaptada de Whitney et al. (2003). 
Os 200 mil modelos produzidos por ROB07 encontram-se disponíveis sob a forma de uma ferramenta online de ajuste de SEDs 2. Para utilizá-la, basta incluir as informações de fluxo, abertura da fotometria, distância e extinção visual. As duas últimas grandezas devem ser expressas sob a forma de valores mínimo e máximo, tornando-as dois parâmetros ajustáveis do modelo. A ferramenta online realiza a busca em sua base de dados as soluções de menor $\chi^{2}$.

No nosso caso particular, utilizamos intervalos de $\sim 20 \%$ em torno dos valores das distâncias fotométricas dos objetos PDS, estimados por Vieira et al. (2003). O objeto PDS340 é o único entre eles contido no catálogo Hipparcos, sendo então adotada a distância fornecida pela paralaxe. As distâncias das candidatas a estrelas massivas encontram-se nas referências da Seção 4.1.2.

As estimativas de $A_{V}$ foram feitas a partir dos mapas de extinção produzidos por Dobashi et al. (2005). Estes autores determinaram a extinção em toda a Galáxia, com base nos dados DSS (Digitized Sky Survey I) e a tradicional técnica de contagem de estrelas. Para cada objeto de nossa amostra, obtivemos o valor de $A_{V}$ sobre suas coordenadas e estimamos uma margem de erro a esta medida baseado no desvio padrão da extinção na região de $1^{\circ} \times 1^{\circ}$ ao seu redor. A Figura 4.6 ilustra o mapa de extinção da região do objeto G31.41+0.31.

\subsubsection{Aproximação de corpo cinza}

Conforme discutido no início do capítulo, a determinação da massa do envoltório realizada a partir da emissão mm/sub-mm (Eq. 4.1) depende não apenas do valor do fluxo, como também dos valores de $R_{d}, \beta$ e $T_{d}$. Além disto, a maioria dos trabalhos na literatura se baseia em um único valor de fluxo para a estimativa desta massa (e.g., Minier et al. 2005; Hill et al. 2005; André et al.|2008).

Inspirados pelo trabalho de Hill et al. (2009), realizamos o ajuste simultâneo dos parâmetros $M_{e n v}, \beta$ e $T_{d}$ para os objetos de nossa amostra. No nosso caso, ao invés de utilizarmos a combinação de uma componente morna e outra fria para reproduzir o formato da

\footnotetext{
${ }^{2}$ http://caravan.astro.wisc.edu/protostars/
} 


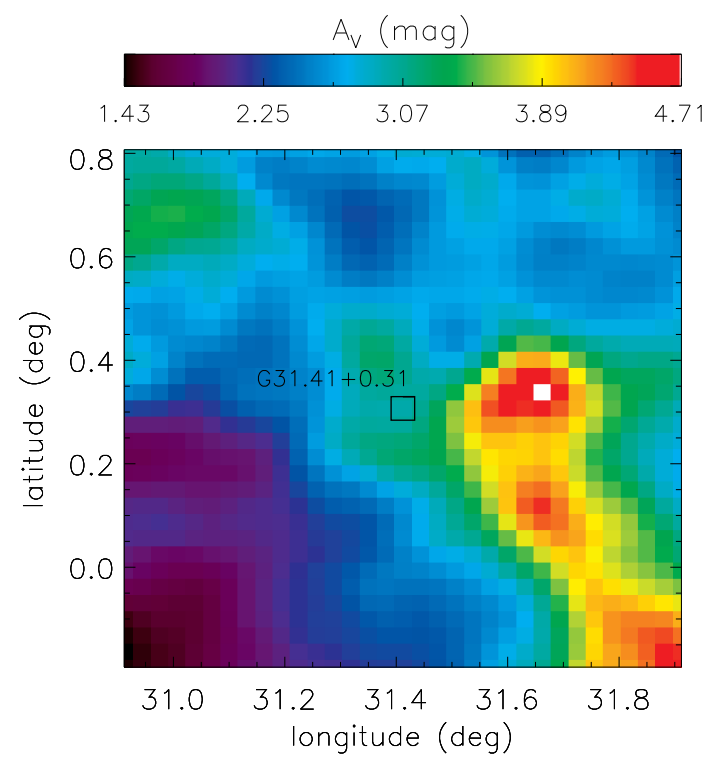

Figura 4.6: Mapa de extinção visual (Dobashi et al., 2005) em região de $0.5^{\circ}$ ao redor do objeto G31.41+0.31.

SED, ajustamos apenas uma componente fria aos comprimentos de onda mais longos $(\lambda \geq 100 \mu \mathrm{m})$. Rearranjando os termos da Equação 4.1 (ou simplesmente tomando apenas o segundo termo da expressão 4.2), temos nossa versão de aproximação de corpo cinza:

$$
F_{\lambda}=\frac{M_{e n v}}{D^{2}} \times \kappa_{0}\left(\frac{\lambda_{0}}{\lambda}\right)^{\beta} \times B_{\lambda}\left(T_{d}\right) .
$$

O ajuste dos parâmetros foi feito através do método de algoritmos genéticos, descrito no Capítulo 3. Adotamos $0 \leq M_{\text {env }} / \mathrm{M}_{\odot} \leq 2000,1 \leq \beta \leq 3$ e $10 \leq T_{d} / 1 \mathrm{~K} \leq 100$ como os intervalos admissíveis para nossos parâmetros. Devido à impossibilidade de desacoplarmos a determinação de $M_{e n v}$ e $R_{d}$, fixamos em 100 a razão entre as massas de gás e da poeira. Adotamos o valor fiducial de opacidade $\kappa_{1.2 \mathrm{~mm}}=1 \mathrm{~cm}^{2} / \mathrm{g}$, o qual é compatível com o trabalho de Ossenkopf e Henning (1994) sobre ambientes circunstelares de poeira. A dependência da distribuição de energia com os parâmetros $T$ e $\beta$ é exemplificada na Figura 4.7, para valores fixos de $M_{e n v}=10 \mathrm{M}_{\odot}$ e $D=1 \mathrm{pc}$.

Os resultados obtidos por esta aproximação são apresentados na próxima seção, onde 

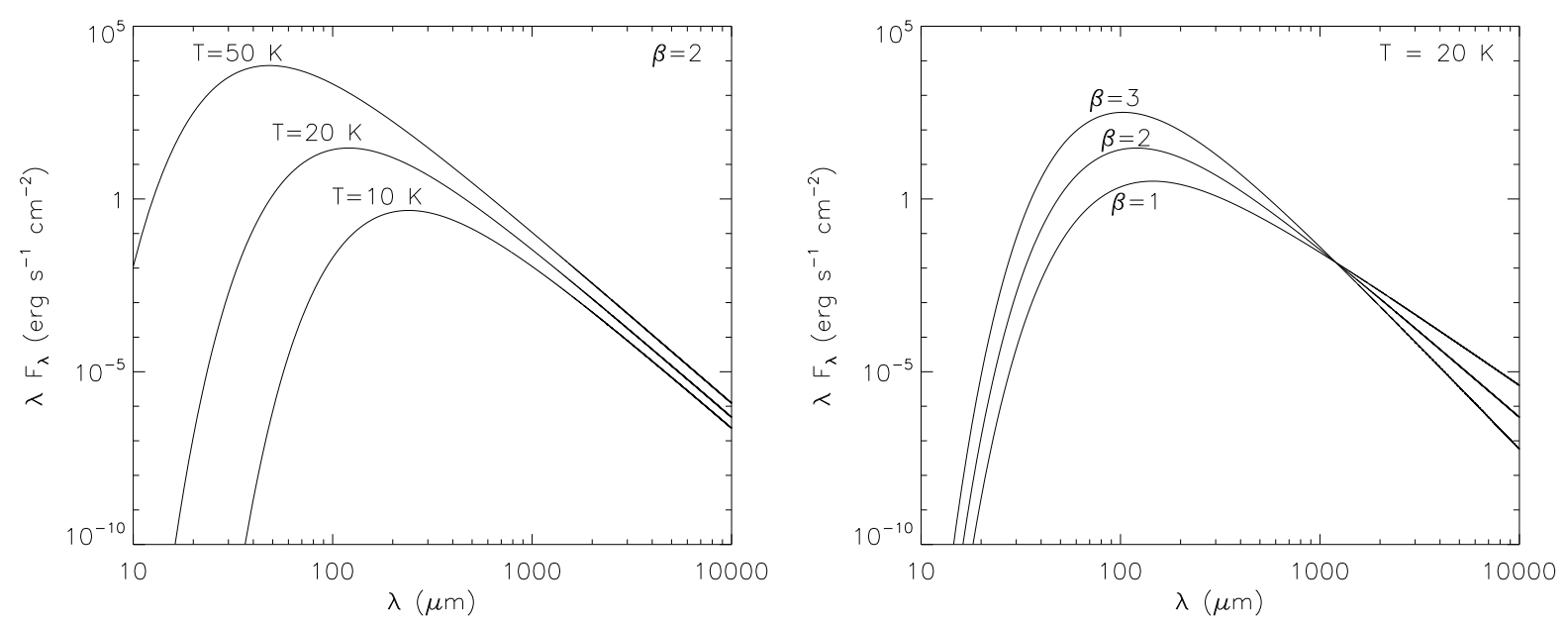

Figura 4.7: Dependência da emissão de corpo cinza com os parâmetros $T$ e $\beta$. Em ambos os casos, temos os valores fixos de $M_{e n v}=10 \mathrm{M}_{\odot}$ e $D=1 \mathrm{pc}$.

eles são comparados com aqueles produzidos pelo modelo de ROB07. Ambas as receitas utilizadas nos levam ao diagnóstico do estágio evolutivo de nossos objetos, realizado através do diagrama $L_{b o l} \times M_{e n v}$.

\subsection{Resultados}

Conforme descrito nas seções anteriores, realizamos o ajuste das distribuições de energia dos objetos de nossa amostra através do modelo de ROB07. Entre os vários parâmetros determinados por sua ferramenta online, temos os valores de $L_{b o l}$ e $M_{e n v}$. A fim de melhor lidarmos com as eventuais degenerecências presentes em modelos de SED, utilizamos os resultados dos 10 melhores ajustes (i.e., de menor $\chi^{2}$ ) encontrados para cada objeto. Desta forma a dispersão dos valores de cada parâmetro pode servir como uma estimativa da incerteza dos valores encontrados.

É importante lembrarmos que a definição do diagrama $L_{b o l} \times M_{e n v}$ descrita por André et al. (2000) exige que esta massa seja "normalizada" para seu valor contido em um diâmetro de 6000 AU. Para tal estimativa, supomos que o densidade dos envoltórios obedeça o perfil $\rho_{\text {env }} \propto r^{-2}$, o que nos leva à expressão linear para a massa: 


$$
M_{e n v}(6000 \mathrm{AU})=M_{e n v} \times\left(\frac{6000 \mathrm{AU}}{2 R_{e n v}}\right)
$$

A Tabela 4.3 exibe os valores mínimos e máximos para $L_{b o l}, M_{e n v}$ (total) e $R_{e n v}$ entre as 10 melhores soluções para cada objeto. Na mesma tabela, podemos encontrar os valores de $A_{V}$ utilizados no ajuste, obtidos através dos mapas de extinção de Dobashi et al. (2005) (Seção 4.2.1), e as distâncias determinadas pelos modelos de menor $\chi^{2}$.

Tabela 4.3 - Resultados do ajuste da SED dos objetos de nossa amostra com o modelo de ROB07. Os valores de $A_{V}$ foram obtidos a partir dos mapas de extinção de Dobashi et al. (2005). As distâncias listadas são referentes às soluções de menor $\chi^{2}$ obtidas pelo ajuste. No caso particular do objeto PDS340, nenhum entre os 10 melhores ajustes indicou a presença de um envoltório circunstelar.

\begin{tabular}{|c|c|c|c|c|c|c|c|c|}
\hline & $\begin{array}{c}A_{V} \\
(\mathrm{mag})\end{array}$ & $\begin{array}{c}D \\
(\mathrm{pc})\end{array}$ & $L_{b o l}^{\min }$ & $\begin{array}{l}L_{b o l}^{\max } \\
\odot)\end{array}$ & $M_{e n v}^{\min }$ & $\begin{array}{l}M_{e n v}^{\max } \\
\odot)\end{array}$ & $\left(\mathrm{R}_{\odot}\right)$ & $\begin{array}{l}R_{e n v}^{\max } \\
\odot)\end{array}$ \\
\hline PDS018 & $2.4 \pm 0.3$ & 360 & 29 & 113 & 0.03 & 0.9 & 1431 & 9101 \\
\hline PDS174 & $1.2 \pm 0.1$ & 1900 & 1257 & 11310 & 362.9 & 2010 & 39460 & 100000 \\
\hline PDS204 & $2.4 \pm 0.1$ & 2450 & 2139 & 2924 & 224.6 & 1306 & 65260 & 100000 \\
\hline PDS207 & $2.3 \pm 0.1$ & 550 & 29 & 133 & 1.9 & 115.2 & 1840 & 46850 \\
\hline PDS216 & $0.8 \pm 0.1$ & 3020 & 357 & 1208 & 4.0 & 183.7 & 5795 & 86180 \\
\hline PDS241 & $0.7 \pm 0.1$ & 3470 & 5114 & 22180 & 729.2 & 3003 & 48280 & 100000 \\
\hline PDS249 & $2.4 \pm 0.1$ & 2000 & 415 & 1442 & 15.1 & 127.1 & 71440 & 100000 \\
\hline PDS290 & $1.7 \pm 0.1$ & 1510 & 193 & 2459 & 66.4 & 1306 & 10580 & 100000 \\
\hline PDS340 & $0.9 \pm 0.1$ & 100 & 42 & 123 & - & - & - & - \\
\hline PDS518 & $5.4 \pm 0.3$ & 200 & 205 & 890 & 9.2 & 134.8 & 6254 & 100000 \\
\hline PDS530 & $1.0 \pm 0.1$ & 1000 & 34 & 330 & 0.6 & 104.3 & 1178 & 31380 \\
\hline G31.41+0.31 & $3.0 \pm 0.6$ & 8320 & 3418 & 142800 & 126.8 & 2488 & 16160 & 100000 \\
\hline RCW36\#1 & $2.8 \pm 0.5$ & 850 & 738 & 4902 & 62.1 & 1522 & 14590 & 100000 \\
\hline RCW36\#2 & $2.8 \pm 0.5$ & 900 & 1546 & 5138 & 16.2 & 672.2 & 21020 & 100000 \\
\hline G265.3+1.43 & $3.2 \pm 0.6$ & 580 & 52 & 230 & 10.6 & 402.8 & 5664 & 40930 \\
\hline
\end{tabular}

A faixa espectral de $\lambda \geq 100 \mu \mathrm{m}$ foi também ajustada por nossa aproximação de corpo cinza, consistindo em uma determinação alternativa de $M_{e n v}$. Calculamos também a integral numérica das SEDs disponíveis, de modo a estimar a luminosidade bolométrica dos objetos de nossa amostra de forma independente de modelos. 
No caso dos modelos de ROB07, o raio do envoltório é um dos parâmetros determinados pelos melhores ajustes. Já para as estimativas de massa obtidas com a aproximação de corpo cinza, nós calculamos o valor de $R_{e n v}$ através do truncamento do valor de $\rho_{e n v}$. Como valor mínimo da densidade, escolhemos um limite superior típico do meio interestelar, $\rho_{\text {lim }}=1.67 \times 10^{-18} \mathrm{~g} / \mathrm{cm}^{3}$ (equivalente a $n_{H_{2}}=5 \times 10^{5} \mathrm{~cm}^{-3}$ ). Explicitamente, temos as relações:

$$
\rho_{e n v}(r)=\frac{1}{4 \pi r^{2}} \frac{d M(r)}{d r}=\frac{1}{4 \pi r^{2}} \frac{M_{\text {env }}}{R_{e n v}} \stackrel{r=R_{e n v}}{\Longrightarrow} R_{e n v}=\left(\frac{M_{e n v}}{4 \pi \rho_{l i m}}\right)^{1 / 3}
$$

Os parâmetros determinados pelo ajuste podem ser encontrados na Tabela 4.4, A Figura 4.8 exibe os ajustes produzidos por ambos os métodos descritos, sendo neste caso apenas o melhor modelo de ROB07 é mostrado nos gráficos. Finalmente, os valores de $M_{e n v}$ e $L_{b o l}$ obtidos por ambos os métodos são confrontados na Figura 4.9.

Tabela 4.4 - Parâmetros determinados pelo ajuste de corpo cinza. A luminosidade bolométrica foi calculada a partir da integração numérica direta da SED, enquanto que o raio do envoltório foi estimado através do truncamento do valor de sua densidade em $\rho_{e n v}=1.67 \times 10^{-18} \mathrm{~g} / \mathrm{cm}^{3}$.

\begin{tabular}{lcccccc}
\hline \hline & $M_{\text {env }}$ & $\begin{array}{c}M_{\text {env }}(6000 \mathrm{AU}) \\
\left(\mathrm{M}_{\odot}\right)\end{array}$ & $\beta$ & $\begin{array}{c}T \\
(\mathrm{~K})\end{array}$ & $\begin{array}{c}L_{\text {bol }} \\
\left(\mathrm{L}_{\odot}\right)\end{array}$ & $\begin{array}{c}R_{\text {env }} \\
(\mathrm{AU})\end{array}$ \\
\hline PDS018 & 40.4 & 11.6 & 1.0 & 14.0 & 111 & 10462 \\
PDS174 & 771.1 & 82.8 & 1.3 & 19.5 & 809 & 27952 \\
PDS204 & 123.1 & 24.3 & 1.0 & 28.1 & 2909 & 15161 \\
PDS207 & 5.7 & 3.1 & 1.0 & 25.7 & 71 & 5439 \\
PDS216 & 1107.6 & 105.4 & 1.0 & 14.0 & 608 & 31538 \\
PDS241 & 313.4 & 45.4 & 1.0 & 40.3 & 20082 & 20706 \\
PDS249 & 18.2 & 6.8 & 1.0 & 32.9 & 358 & 8020 \\
PDS290 & 1690.8 & 139.7 & 1.9 & 10.0 & 214 & 36314 \\
PDS340 & 0.0 & 0.0 & 1.3 & 71.3 & 28 & 444 \\
PDS518 & 6.9 & 3.6 & 1.8 & 22.7 & 158 & 5793 \\
PDS530 & 74.6 & 17.4 & 1.6 & 14.3 & 592 & 12830 \\
G31.41+0.31 & 1744.3 & 142.6 & 1.0 & 45.3 & 59820 & 36693 \\
RCW36\#1 & 10.0 & 4.6 & 2.9 & 28.0 & 7966 & 6611 \\
RCW36\#2 & 10.6 & 4.8 & 3.0 & 26.9 & 10451 & 6714 \\
G265.3+1.43 & 32.4 & 10.0 & 1.3 & 20.4 & 42 & 9714 \\
\hline \hline
\end{tabular}



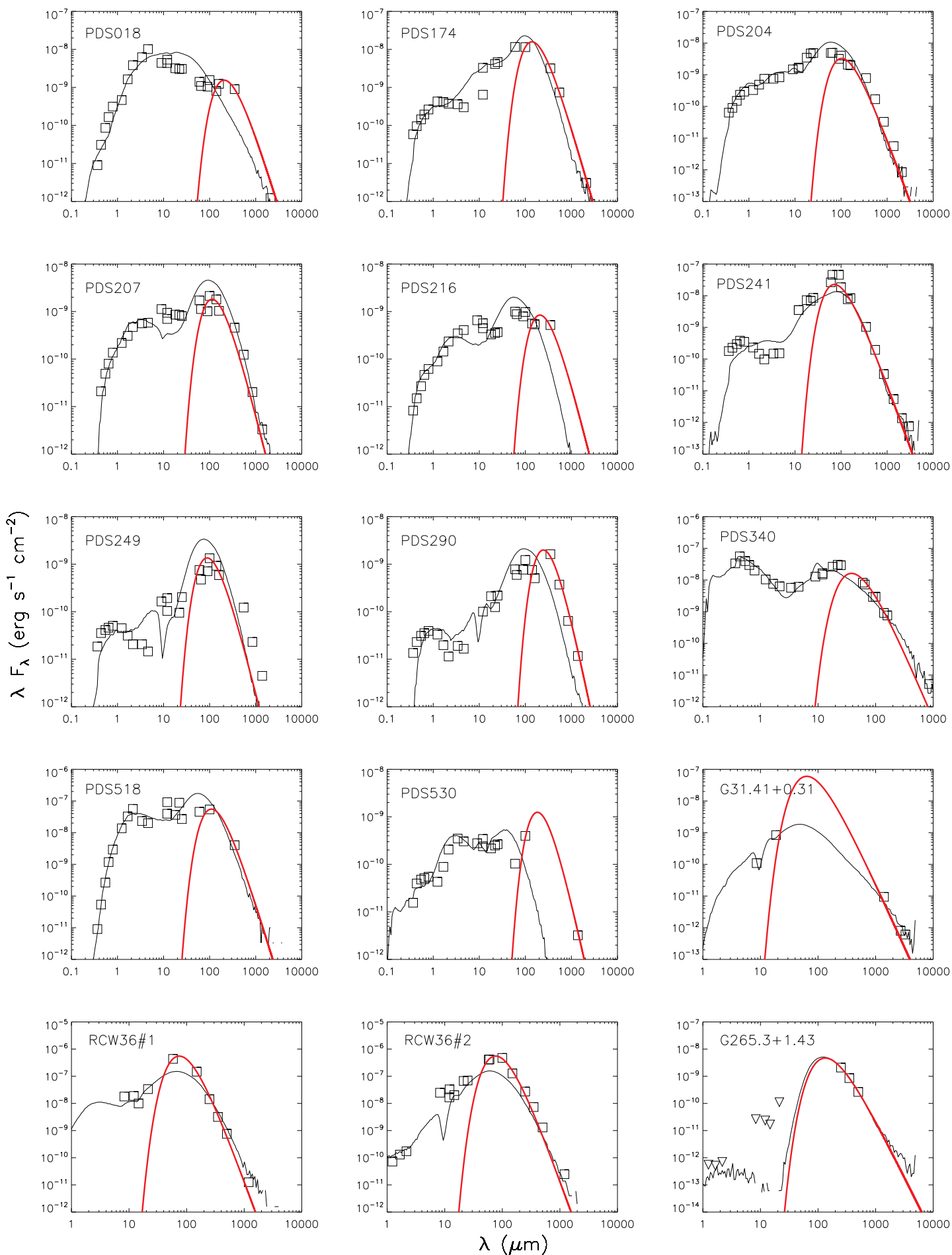

Figura 4.8: Ajustes de SED dos objetos de nossa amostra, através do modelo de ROB07 (curva em preto). A emissão de corpo cinza (curva em vermelho) é ajustada somente aos dados no IR distante. 

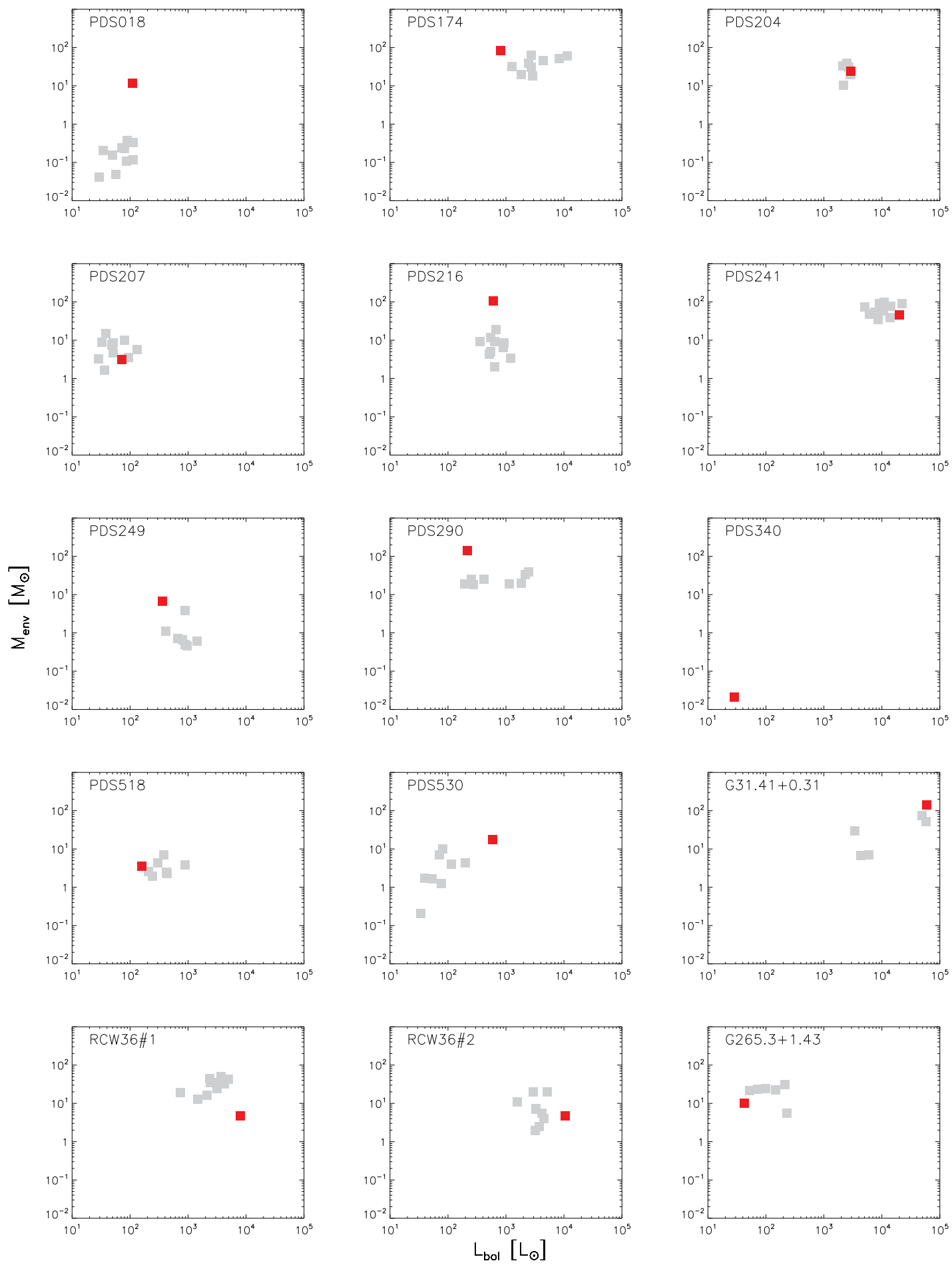

Figura 4.9: Comparação entre os valores de $L_{b o l}$ e $M_{e n v}$ determinados por métodos distintos. Os quadrados em cinza representam os valores dados pelas 10 soluções de menor $\chi^{2}$ dada pela ferramenta online de ROB07. Os quadrados vermelhos representam os valores de massa do envoltório estimados pelo ajuste de corpo cinza aos fluxos do IR distante/mm e a integração direta da SED para o cálculo da luminosidade bolométrica. 


\subsection{Discussão}

Neste ponto, dispomos de valores de $L_{b o l}$ e $M_{e n v}$ obtidos por métodos independentes. O modelo desenvolvido por ROB07 é indiscutivelmente uma ferramenta muito mais sofisticada do que a aproximação de corpo cinza. No entanto, sua complexidade pode se tornar uma desvantagem em certos casos.

O ajuste de SED fornecido pela grade de modelos online é função de 15 parâmetros, o que além de indicar soluções bastante distintas (compare os valores mínimos e máximos apresentados na Tabela 4.3), também exige que o objeto sob estudo disponha de um conjunto de dados que abranja todo o seu domínio espectral. Este problema é menos grave quando estamos interessados apenas no valor específico da massa do envoltório. Neste caso, os fluxos na região entre o IR distante e o milimétrico são os principais responsáveis pela estimativa desta grandeza. Em outras palavras, o valor de $M_{e n v}$ é especialmente dependente da parte mais "fria" da SED.

O ajuste de corpo cinza, embora bastante simples, depende de poucos parâmetros. Além disto, no seu caso apenas a região de interesse da SED é ajustada $(\lambda \geq 100 \mu \mathrm{m})$. Obviamente, sua dedução exige hipóteses bastante simplificadoras, que podem eventualmente divergir das condições físicas reais. No entanto, esta aproximação ainda se trata de uma estimativa bastante útil para melhor compreendermos as propriedades dos envoltórios circunstelares.

A análise conjunta das Figuras 4.8 e 4.9 revela de forma qualitativa a origem das concordâncias e desvios dos resultados finais obtidos por cada método. Podemos observar que os ajustes de SED de ROB07 que reproduzem bem a faixa do mm/sub-mm tendem a concordar melhor com os resultados obtidos pela aproximação de corpo cinza. Este é o caso dos objetos PDS174, PDS204, PDS207, PDS241, PDS518 e G31.41+0.31. Além disto, o baixo valor de $M_{\text {env }}$ determinado para PDS340 pode também ser interpretado como compatível com a ausência do envoltório, conforme indicam os melhores ajustes da base de modelos online. Este resultado concorda com nossas expectativas, dado que este é o único objeto da amostra em um estágio evolutivo mais avançado, de acordo com a classificação 
dada por Sartori et al. (2010). Em particular, os ajustes obtidos para a SED do objeto G3141+0.31 são bastante distintos, devido à ausência de observações que restrinjam os modelos na região do IR médio e distante.

Nos ajustes onde apenas o corpo cinza apresenta boa concordância com os dados observados no mm/sub-mm, o valor da massa do envoltório é geralmente subestimado pelos ajustes de ROB07. Podemos notar este comportamento para PDS018, PDS216, PDS290 e PDS530.

Finalmente, as eventuais diferenças entre os valores de $L_{b o l}$ são principalmente devidas à extrapolação feita pelos modelos de ROB07 nas regiões da SED com poucos vínculos observacionais.

Para construirmos a versão final do diagrama evolutivo $L_{b o l} \times M_{e n v}$, optamos pelos valores obtidos através da aproximação de corpo cinza. A distribuição de nossa amostra sobre este diagrama é apresentada na Figura 4.10.

A característica mais evidente extraída da análise do gráfico é que a maioria dos objetos PDS encontra-se na fase de transição entre a Classe 0 e a Classe I. Este resultado é compatível com o fato desta sub-amostra se tratar do grupo mais embebido de estrelas HAeBe do PDS. Pertencer a este grupo significa estar associado a uma grande quantidade de material circunstelar e ao mesmo tempo já possuir uma contrapartida óptica. No nosso caso, o diagrama mostra que a massa circunstelar já atingiu um valor comparável àquele acumulado pelo objeto central.

Ainda segundo a distribuição no diagrama, os objetos G265.3+1.43, PDS290 e PDS216 parecem se encontrar em estágios evolutivos anteriores ao dos outros objetos, enquanto que PDS340, RCW36\#1 e RCW36\#2 mostram indícios de uma estrutura circunstelar mais evoluída.

Entre as candidatas a estrelas massivas, o objeto G31.41+0.31 se confirmou como uma 


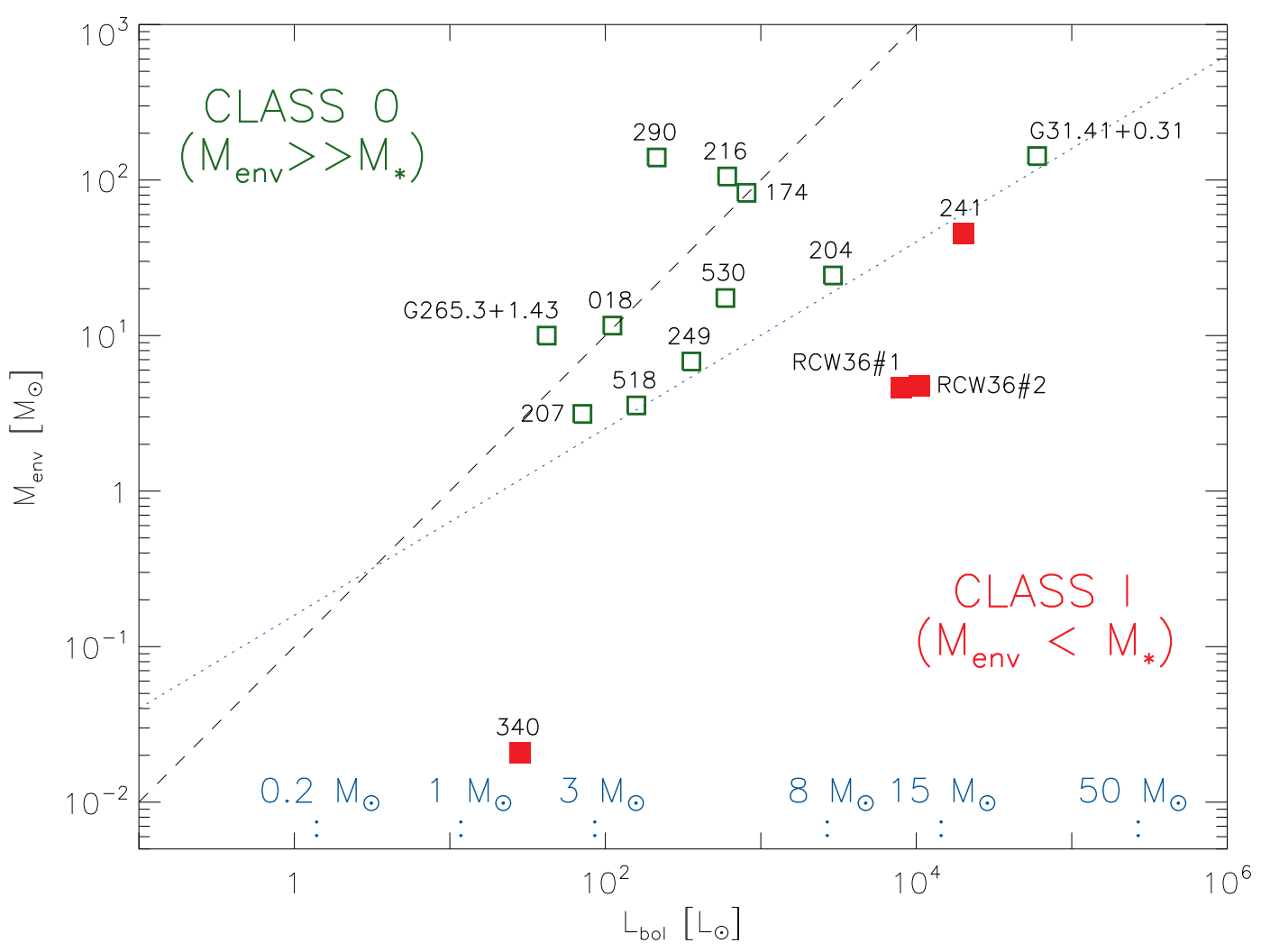

Figura 4.10: Distribuição da sub-amostra PDS e candidatas a estrelas massivas sobre o diagrama de $L_{b o l} \times M_{e n v}$. Para maiores detalhes, reveja a descrição da Figura 4.2 .

protoestrela com possível massa final realmente elevada $\left(\gtrsim 50 \mathrm{M}_{\odot}\right)$. Os objetos situados na região de RCW36 parecem estar em um regime de massa apenas um pouco acima da faixa das estrelas HAeBe, entre $10 \mathrm{M}_{\odot}$ e $15 \mathrm{M}_{\odot}$. Já candidata G265.3+1.43 se encontra em uma posição compatível com a massa de uma estrela do tipo HAeBe típica.

Finalmente, o objeto PDS241 se revelou como um objeto bastante massivo, com possível valor final de massa maior do que $15 \mathrm{M}_{\odot}$. Vieira et al. (2003) apontam esta fonte como sendo do tipo espectral B0, o que também indica uma natureza massiva. 
Capítulo 5

\section{Morfologia no infravermelho médio}

Na última década, as técnicas de observação no infravermelho (IR) médio feita a partir do solo se consolidaram como uma importante ferramenta para o estudo de estrelas jovens. Esta faixa espectral se estende entre $8 \mu \mathrm{m}$ e $30 \mu \mathrm{m}$, permitindo a observação de poeira morna $(100-400 \mathrm{~K})$ presente nos mais diversos ambientes astrofísicos. No caso de discos circunstelares, este intervalo corresponde a distâncias da ordem de uma unidade astronômica do objeto central, sendo portanto ideal para o estudo da região do disco mais próxima da estrela.

No caso de observações feitas a partir do solo, contamos com as janelas atmosféricas correspondentes às bandas $N$ e $Q$, representadas na Figura 5.1. A grande vantagem das observações terrestres é justamente o grande porte dos telescópios, que atualmente chegam a 8-10 m de diâmetro (e.g., Gemini, VLT, Keck, Subaru) e encontram-se nos melhores sítios astronômicos conhecidos (i.e., ótimas condições de seeing). Isto faz com que a resolução espacial destas observações sejam limitadas apenas pelo limite de difração do aparato óptico, proporcional a $\lambda / D$. Para comprimentos de onda no óptico e no IR próximo, o efeito da turbulência atmosférica sobre a resolução espacial de uma imagem é mais relevante do que o limite de difração. No entanto, o valor do seeing decresce para maiores comprimentos de onda $\left(\propto \lambda^{-0.2}\right)$, tornando-o menos importante para observações no IR médio. A Figura 5.2 exibe a dependência espectral da resolução angular de vários instrumentos que operam no IR, além das curvas de resolução limite para seeings de $0.5^{\prime \prime}$ e $1^{\prime \prime}$.

Apesar do grande poder de resolução espacial, as observações terrestres no IR médio 


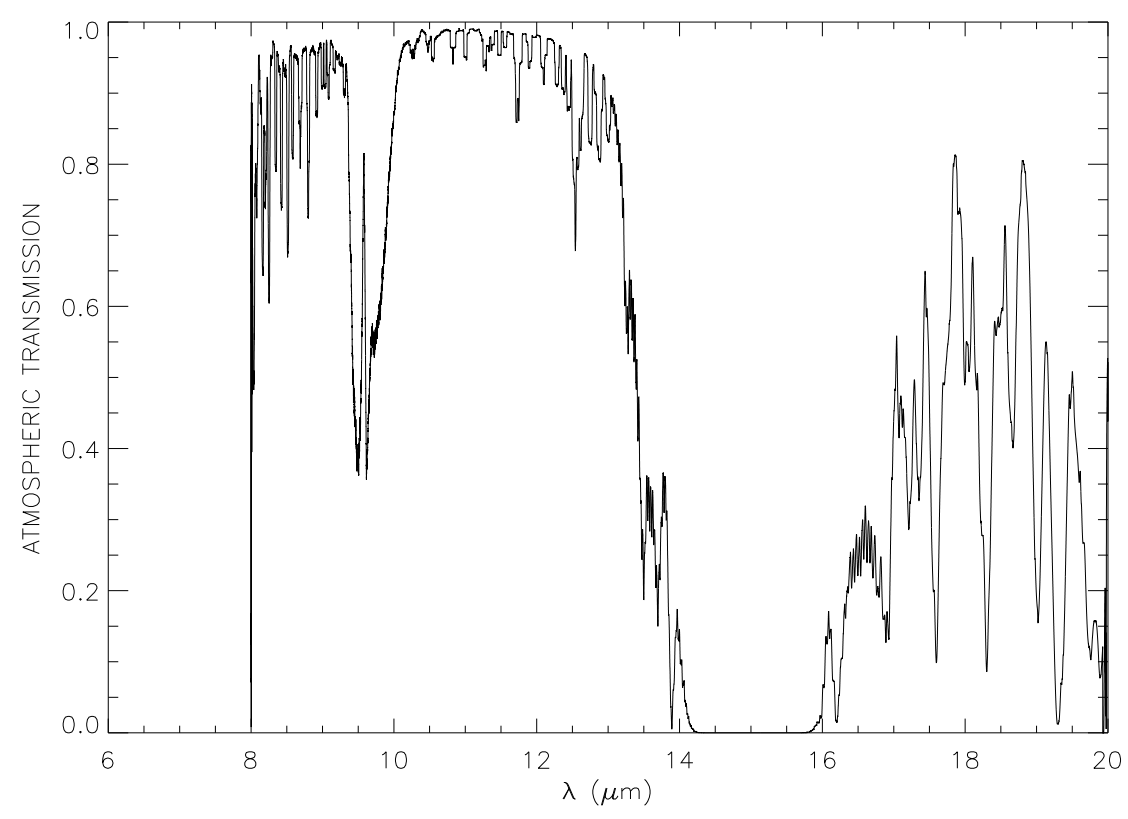

Figura 5.1: Transmissão atmosférica calculada pelo código ATRAN (Lord, 1992). A banda $N$ encontra-se aproximadamente entre $8 \mu \mathrm{m}$ e $14 \mu \mathrm{m}$, enquanto que a banda $Q$ está situada entre $16 \mu \mathrm{m}$ e $20 \mu \mathrm{m}$.

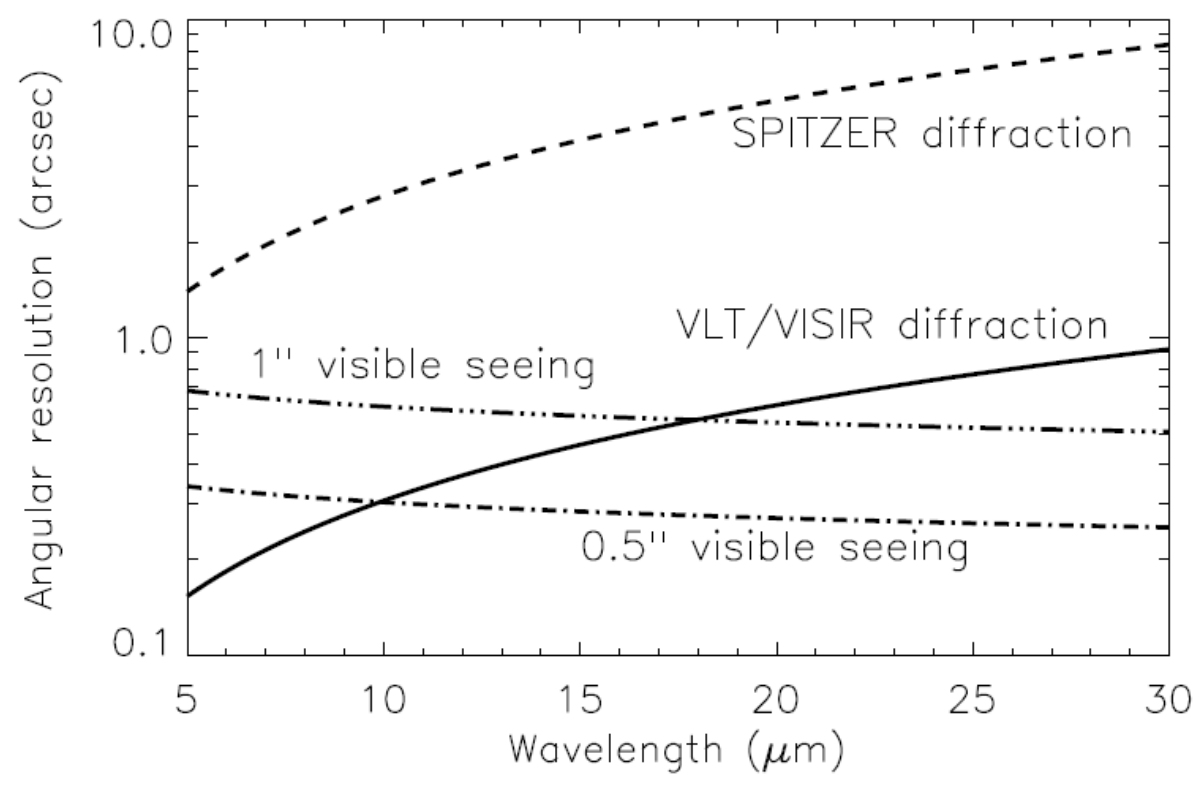

Figura 5.2: Resolução espacial de missões astronômicas que operam no IR, comparadas às curvas de seeing óptico igual a $0.5^{\prime \prime}$ e a $1^{\prime \prime}$. As curvas de seeing seguem a fórmula de Roddier, expressa por seeing $\propto \lambda^{-0.2}$. Figura adaptada do "VISIR User Manual", disponível na página do ESO (European Southern Observatory). 
são extremamente afetadas pela ação atmosférica, o que não ocorre com os observatórios espaciais. Disto decorre a complementaridade entre estas duas modalidades de observação: detecções feitas a partir do espaço ganham em sensibilidade, por não estarem sujeitas aos efeitos atmosféricos, enquanto que observações terrestres contam com espelhos primários de maior porte, que garantem uma maior resolução espacial. Para se ter uma ideia de ordens de grandeza, o satélite SPITZER possui um telescópio com abertura de $90 \mathrm{~cm}$, o qual lhe rende uma resolução angular de $2.2^{\prime \prime}$ em $8 \mu \mathrm{m}$. Já o VLT (Very Large Telescope) apresenta um espelho primário com diâmetro de $8.2 \mathrm{~m}$, o que lhe permite atingir resoluções espaciais de até $0.25^{\prime \prime}$. A Figura 5.3 compara a sensibilidade do instrumento VISIR ( VLT spectrometer and imager for the mid-infrared) com algumas missões espaciais.

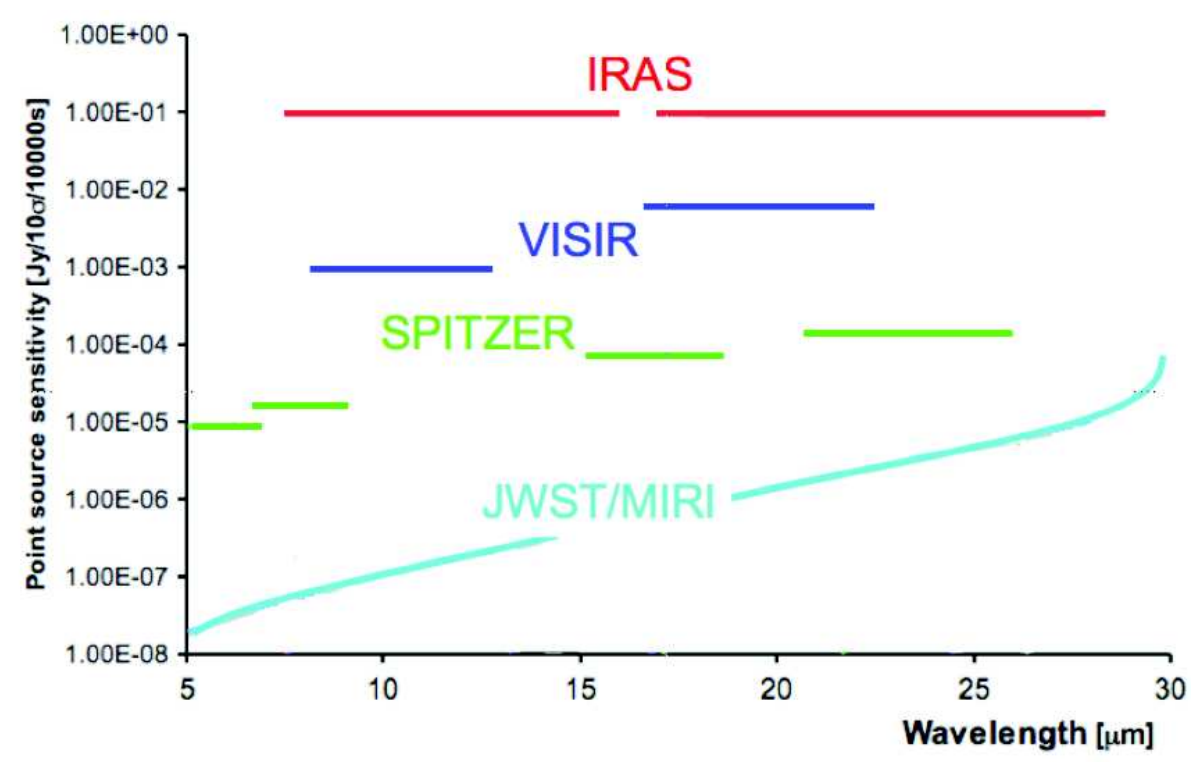

Figura 5.3: Comparação entre a sensibidade dos detectores de algumas missões que operam no IR. Figura adaptada de Pantin (2011).

\subsection{Observações VISIR}

No Capítulo 4 apresentamos algumas observações obtidas através do instrumento VISIR, abrigado no VLT. No entanto, naquele contexto os dados já estavam reduzidos e devidamente calibrados em fluxo. Neste capítulo, descreveremos mais a fundo este instrumento do VLT, lidando diretamente com o processo de redução e análise de suas observações. Uma série de ferramentas próprias foi desenvolvida nesta etapa do trabalho, produzindo 
resultados bastante interessantes.

\subsubsection{Objeto de estudo: PDS340}

Afim de aprofundarmos nosso conhecimento sobre as estrelas HAeBe de nossa amostra, buscamos observações adicionais no banco de dados VISIR (ESO Archive). Cerca de vinte objetos deste grupo possuem dados disponíveis nesta base, tanto de imageamento como espectroscópicos. Para o desenvolvimento de ferramentas de redução e análise destes dados, elegemos as imagens do objeto PDS340, por se tratar de um dos alvos mais próximos de nossa lista (103 pc). Tanto a espectroscopia deste objeto quanto a redução e análise dos dados disponíveis para os outros objetos são deixados neste ponto como uma perspectiva bastante interessante para trabalhos futuros.

Por se tratar de um objeto relativamente próximo, PDS340 (também conhecido como HD 100546) já foi alvo de inúmeros estudos na literatura. Pantin et al. (2000) observaram sua estrutura circunstelar nas bandas $J$ e $K_{S}$, utilizando óptica adaptativa. Este estudo revelou um pico de densidade a $40 \mathrm{AU}$ do objeto central, uma inclinação do sistema de $50^{\circ} \pm 5^{\circ}$ e uma extensão total do disco de 200 AU nestas bandas.

Observações coronográficas de PDS340 foram realizadas com o instrumento NICMOS2 $\left(\lambda_{c}=1.6 \mu \mathrm{m}\right)$ do HST (Hubble Space Telescope-Augereau et al. 2001), levando a resultados similares àqueles obtidos por Pantin et al. (2000). Utilizando observações coronográficas obtidas pelo HST em bandas ópticas, Ardila et al. (2007) obtiveram uma inclinação do sistema de $42^{\circ} \pm 5^{\circ}$ e um ângulo de posição de $161^{\circ} \pm 5^{\circ}$ (do Leste para o Norte). Além disto, estas observações detectaram algumas sub-estruturas no disco, como por exemplo os "braços espirais" previamente documentados por Grady et al. (2001).

Benisty et al. (2011) reuniram em seu trabalho observações interferométricas obtidas com os intrumentos AMBER e MIDI do VLTI (Very Large Telescope Interferometer) e medidas fotométricas disponíveis para PDS340. O modelo proposto por estes autores para ajustar todo o conjunto de dados inclui (i) um disco próximo à estrela, composto por grãos com tamanhos entre $0.1 \mu \mathrm{m}$ e $5 \mu \mathrm{m}$; (ii) uma região vazia entre 4 AU e 13 AU; e (iii) 
um disco massivo com grãos menores em sua superfície. A Figura 5.4 ilustra a estrutura descrita por Benisty et al. (2011).
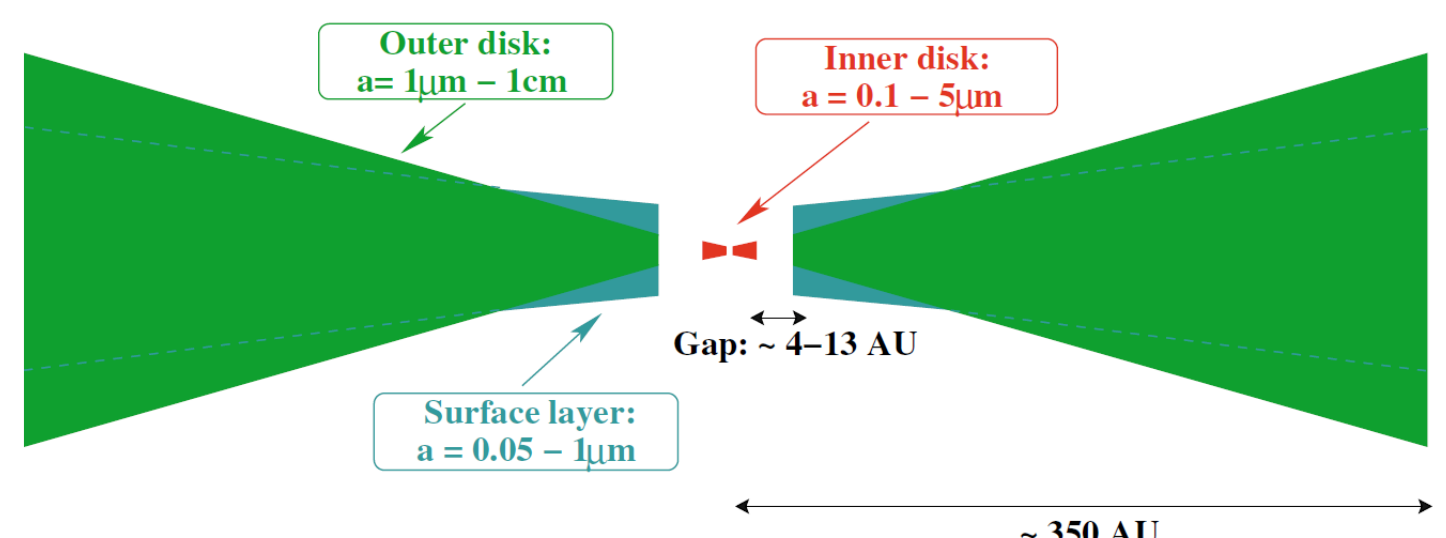

Figura 5.4: Estrutura de disco de PDS340 proposta por Benisty et al. (2011). Figura extraída do mesmo trabalho.

\subsubsection{Descrição do instrumento}

O instrumento VISIR opera em modos de imageamento e espectroscopia, no intervalo entre $8 \mu \mathrm{m}$ e $20 \mu \mathrm{m}$. Ele está posicionado no foco Cassegrain do UT3 do VLT, no Cerro Paranal (Chile). Seu imageamento possui resolução limitada por difração $\left(\sim 0.3^{\prime \prime}\right.$ na banda $N)$ e seu espectrógrafo dispõe das opções de baixa e média resoluções espectrais $(R=150$ e 3000, respectivamente). Ambas as opções contam com um detector DRS $256 \times 256$ BIB, com eficiência quântica de $70 \%$ na banda $N$.

A atmosfera terrestre não apenas absorve a maior parte da radiação no IR médio, como também emite fortemente como um corpo negro a cerca de $253 \mathrm{~K}$. Além deste efeito, o próprio telescópio emite termicamente nesta região espectral, com uma temperatura típica de $283 \mathrm{~K}$. O instrumento VISIR é refrigerado, de forma a evitar contaminação interna nas observações. O fundo de radiação em $10 \mu \mathrm{m}$ é de cerca de $3700 \mathrm{Jy} / \operatorname{arcsec}^{2}$ e em $20 \mu \mathrm{m}$ é de $8300 \mathrm{Jy} / \operatorname{arcsec}^{2}$, gerando um fluxo de fótons frequentemente superior a $10^{8} \mathrm{~s}^{-1}$. Estes altos valores acabam por restringir o tempo de integração de uma exposição a algumas dezenas de mili-segundos. 
As técnicas de chopping e nodding são ideais para este caso onde se deseja suprimir o brilho de fundo. Na técnica de chopping, uma sequência de duas observações é efetuada: uma sobre a fonte e outra do céu subjacente. Esta sequência deve ser mais rápida do que a escala de tempo das flutuações do fundo de radiação, o que na prática é possível através do movimento do espelho secundário do telescópio. A frequência típica de oscilação deste espelho para observações na banda $N$ é de $0.25 \mathrm{~Hz}$, enquanto que o valor de $0.5 \mathrm{~Hz}$ é adotado para o imageamento na banda Q. Observações espectroscópicas exigem menores frequências de oscilação, da ordem de $0.1 \mathrm{~Hz}$.

O chopping remove a maior parte do brilho de fundo. No entanto, o caminho óptico não é exatamente o mesmo para ambas as posições imageadas, levando à persistência de um fundo de radiação residual. Este resíduo é produzido pela variação da temperatura do telescópio e portanto possui uma escala de tempo mais longa do que as flutuações do céu. Para sua supressão, faz-se uso da técnica que conhecemos por nodding.

O nodding nada mais é do que o reposicionamento do próprio telescópio sobre e fora da fonte. Este apontamento também é periódico, ocorrendo a cada 30 - 60 s tipicamente. Desta forma, temos na prática uma sequência de chopping para cada posição de nodding, de forma a eliminar tanto o brilho de fundo de curta escala de tempo (atmosférico) como também o de mais longa escala de tempo (oriundo do telescópio). O esquema completo pode ser visualizado na Figura 5.5.

Se a cobertura do campo é suficientemente grande e as amplitudes de oscilação do chopping e nodding são nele contidas, múltiplas imagens de um mesmo objeto estarão presentes em um mesmo plano. Se a oscilação de chopping for perpendicular à de nodding, até quatro imagens podem estar contidas no campo. No caso das oscilações paralelas, até três imagens do alvo podem estar no campo. A Figura 5.6 ilustra ambas as possibilidades de direções oscilatórias. 


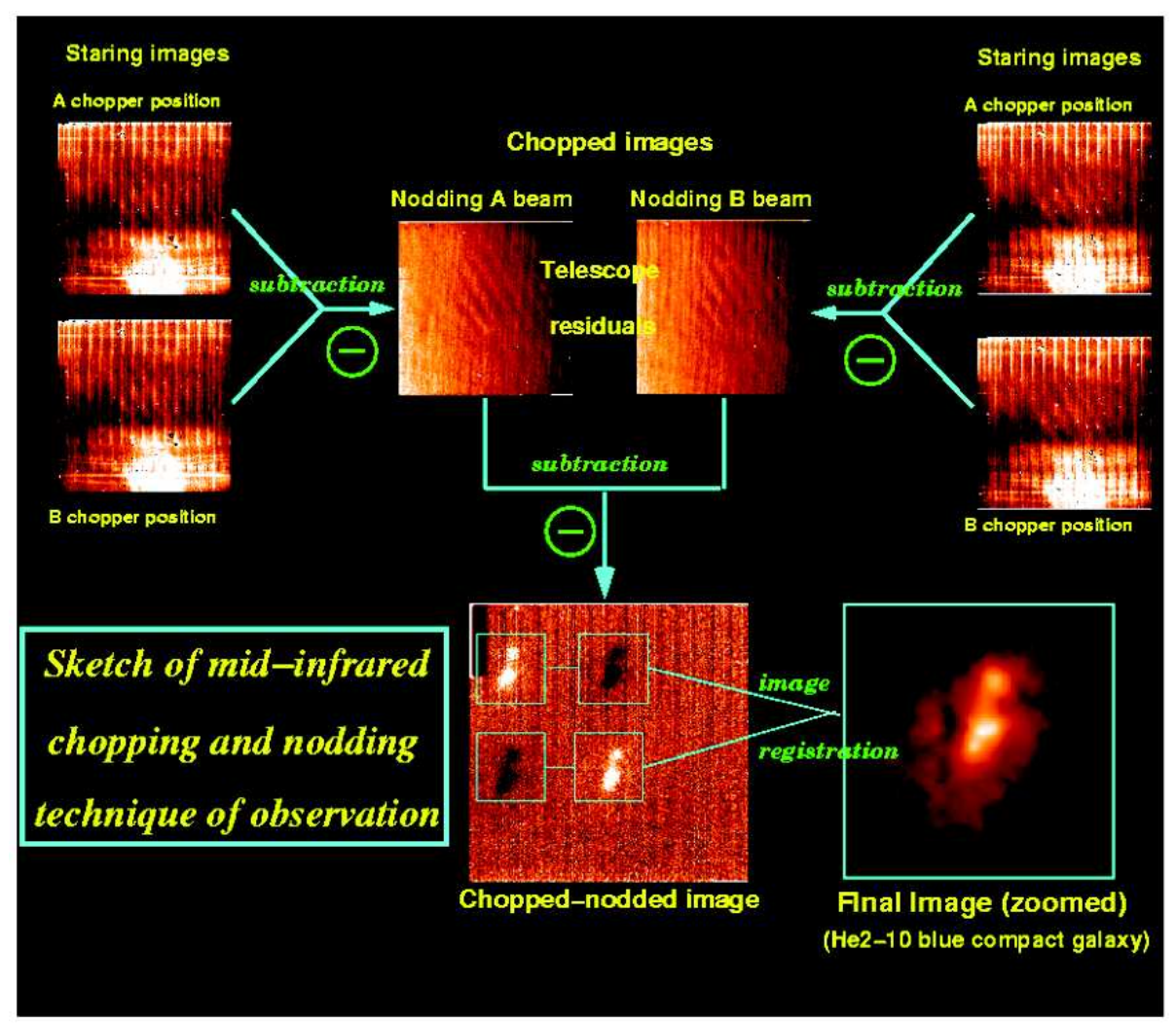

Figura 5.5: Esquema da utilização das técnicas de chopping e nodding nas observações feitas com o instrumento VISIR. Neste caso as oscilações de chopping e nodding são perpendiculares entre si, gerando quatro imagens do alvo contidas no mesmo campo de visão. Figura adaptada de Pantin (2011).
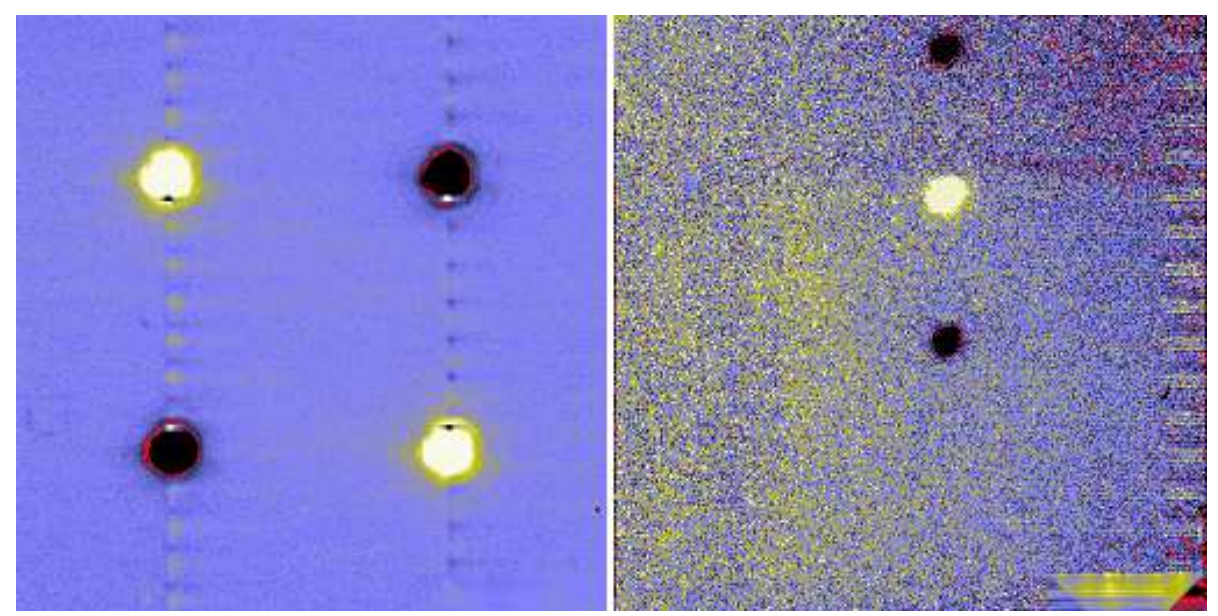

Figura 5.6: Ilustração dos modos perpendicular (à esquerda) e paralelo (à direita) de oscilação utilizados na técnica de chopping e nodding com o instrumento VISIR. O objeto imageado em ambos os casos é PDS061, nas bandas PAH1 (esquerda, $8.59 \mu \mathrm{m}$ ) e Q2 (direita, $18.72 \mu \mathrm{m}$ ). 


\subsection{Redução dos dados}

\subsubsection{Descrição das imagens brutas}

A utilização das técnicas de chopping e nodding torna a organização dos dados obtidos pelo instrumento VISIR bastante particular. Conforme dito na seção anterior, o enorme fundo de radiação obriga que a unidade de tempo de integração (DIT) seja da ordem de algumas dezenas de mili-segundos. O valor mínimo para o DIT é de $5 \mathrm{~ms}$, o que implica na leitura de 200 quadros por segundo. Como este volume de dados é grande demais para ser armazenado, na prática as exposições elementares são somadas em tempo real para cada ciclo de chopping. A sequência de uma dada observação realizada pelo instrumento VISIR é esquematizada na Figura 5.7 .

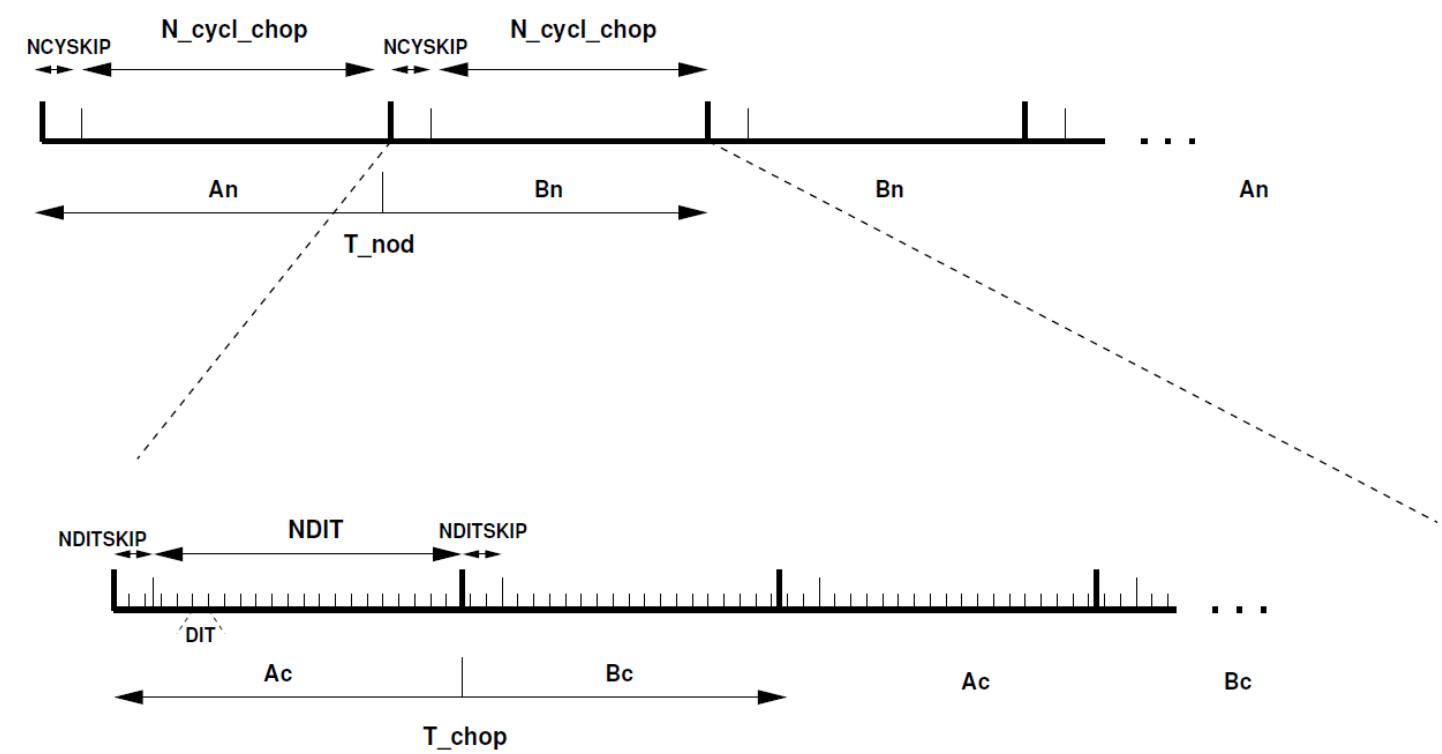

Figura 5.7: Sequência cronológica de uma observação VISIR. Ac e Bc referem-se a duas posições de chopping, enquanto que An e Bn a duas posições de nodding (i.e., do telescópio).

Figura adaptada do "VISIR User Manual".

Desta forma, temos uma imagem para cada ciclo de chopping, o qual por sua vez é constituído por uma exposição "sobre" a fonte e outra "fora" da fonte (note que o emprego desta terminologia - do inglês "on-" e "off-source" - não é completamente preciso, dado que na maioria das vezes o alvo encontra-se contido no campo do detector durante toda a sequência de aquisição). Cada posição de nodding pode conter vários ciclos de chopping, 
e assim temos como produto final uma série de observações de um mesmo objeto. Este conjunto de imagens brutas é armazenado sob a forma de um cubo de dados de extensão fits. Por sua vez, este cubo contém $2 n+1$ planos, sendo $n$ o número total de ciclos de chopping. Isto quer dizer que para cada um destes ciclos, duas imagens são armazenadas no cubo de observações. Sendo $A$ e $B$ representantes dos semi-ciclos "sobre" e "fora" da fonte, os dois primeiros planos contêm:

- A imagem $A_{1}$, correspondente à primeira metade do ciclo de chopping;

- A diferença entre os dois semi-ciclos, $A_{1}-B_{1}$.

Similarmente, temos para o i-ésimo ciclo:

- A $i$-ésima primeira metade do ciclo de chopping, $A_{i}$;

- A média das diferenças entre todos os semi-ciclos até então observados, $\left[\left(A_{1}-B_{1}\right)+\right.$ $\left.\left(A_{2}-B_{2}\right)+\ldots+\left(A_{i}-B_{i}\right)\right] / i$.

Finalmente, o último plano contido no cubo de dados é a média das diferenças entre todos os semi-ciclos da sequência completa de aquisição, ou seja, simplesmente uma cópia do plano anterior. Para cada posição de nodding (i.e., para cada apontamento do telescópio) é associado um arquivo de extensão fits independente.

\subsubsection{Rotinas de redução}

O ESO fornece um conjunto de rotinas destinadas à organização e redução dos dados VISIR. No entanto, este pipeline possui funcionalidade limitada e problemas recorrentes com artefatos produzidos pelo instrumento. Por esta razão, desenvolvemos durante o trabalho um conjunto de ferramentas próprias para a redução dos dados VISIR. Em resumo, as tarefas desempenhadas por estas rotinas escritas em IDL (Interactive Data Language 1 ) são:

- Correção dos artefatos gerados pelo instrumento (veja exemplos nas Figuras 5.8, e 5.9);

\footnotetext{
${ }^{1}$ http://www.exelisvis.com/
} 
- Combinação das imagens "positivas" e "negativas" contidas no último plano de cada arquivo fits (i.e., para cada posição de nodding);

- Combinar as observações de todas as posições de nodding;

- Calibrar em fluxo, por meio da comparação entre as contagens do alvo de interesse e de estrelas padrões.

Neste ponto, note-se que a correção de flatfield não é executada durante o processo. Segundo a equipe responsável pelo instrumento, esta correção não é estável com o detector atual, o que torna o procedimento dispensável.

A correção dos artefatos é realizada utilizando-se os preceitos descritos por Pantin (2011). Nesta referência, são reportados tanto o problema de "fastasmas" na imagem como o problema de "estrias". O primeiro é o já bem conhecido efeito Hammer, descrito por Sako et al. (2003). Ele possui natureza eletrônica e é normalmente associado a fontes brilhantes. Já o efeito de "estrias" não possui origem física bem conhecida, e é caracterizado por artefatos aditivos horizontais que se repetem periodicamente na direção vertical. Quando o alvo observado não se trata de uma fonte extensa, a simples subtração da mediana do brilho de cada linha horizontal basta para efetuarmos esta correção.

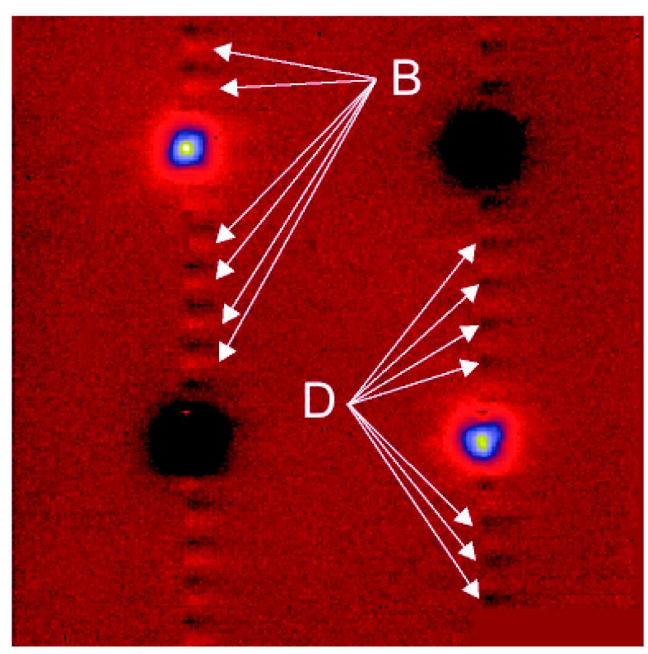

Figura 5.8: Artefatos produzidos pelo efeito Hammer em observação VISIR. Figura adaptada de Pantin (2011). 


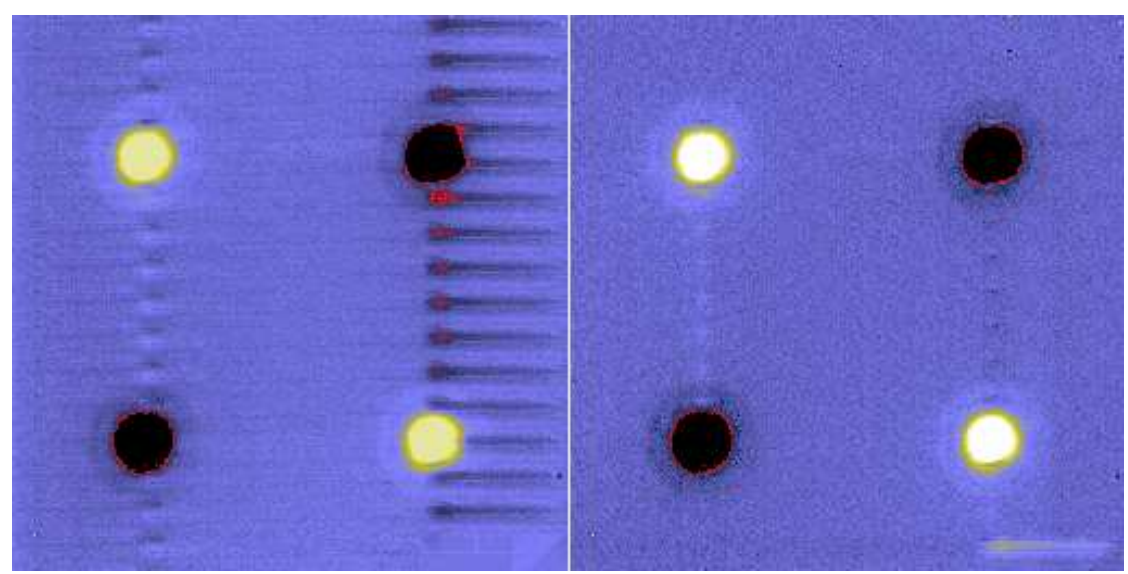

Figura 5.9: Exemplo de aplicação de nossas rotinas para a eliminação de artefatos de uma imagem VISIR. À esquerda temos uma imagem combinada e corrigida pelas rotinas oferecidas pelo ESO, enquanto que à direita temos a mesma imagem após ser submetida às nosssa ferramentas de correção.

As rotinas fornecidas pelo ESO combinam apenas as várias posições de nodding, deixando a cargo do usuário a combinação posterior das múltiplas imagens contidas em um mesmo plano. Esta operação é executada através da simples soma direta das imagens, o que como vimos pode levar a resultados imprecisos.

A combinação das múltiplas imagens contidas em cada plano e a adição entre os resultados de cada posição de nodding constituem um passo essencial e extremamente delicado no processo de redução dos dados. Doucet et al. (2006) demonstram que a simples combinação dos máximos, ou mesmo dos centróides das fontes, pode levar a resultados espúrios (Figura 5.10). Por isso, decidimos efetuar esta operação seguindo os seguintes passos:

- Extrair completamente o fundo de céu ainda presente na imagem;

- Inverter o sinal dos picos de fluxo "negativo" (i.e., aqueles que se encontravam subtraídos nas imagens brutas);

- Posicionar um deles no centro de uma imagem de $64 \times 64$ píxeis (lembrando que a imagem original possui $256 \times 256$ ), de modo a evitar a presença dos outros picos;

- Encontrar o melhor deslocamento para cada um dos outros picos dentro desta imagem, através da minimização da diferença quadrática de seu valor com o primeiro 


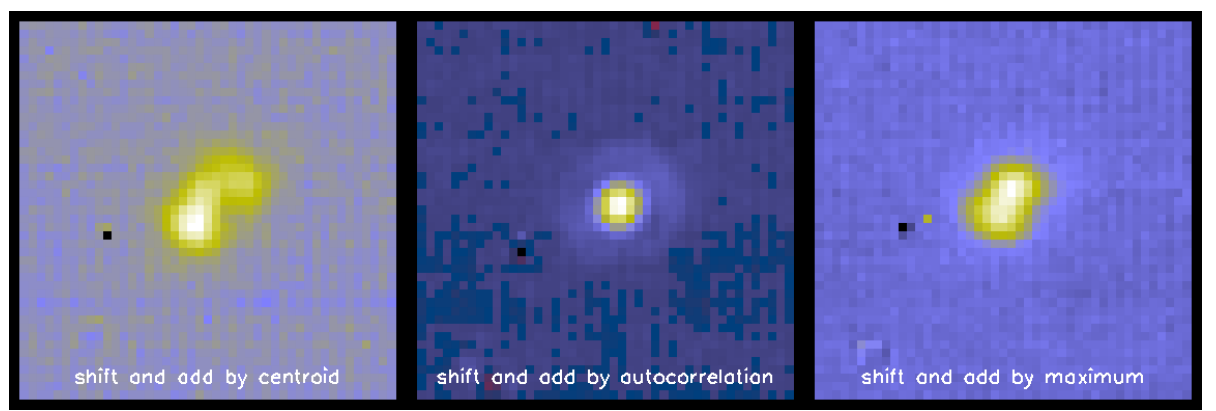

Figura 5.10: Comparação entre diferentes métodos de combinação de imagens VISIR. Na imagem à esquerda foi utilizada a combinação baseada no centróide da fonte. Na imagem central, o método de autocorrelação foi empregado. No painel à direita, a combinação foi feita segundo a posição de máximo da fonte. Figura adaptada de Doucet et al. (2006).

pico. Este deslocamento possui uma precisão de 1/10 de pixel.

O passo seguinte a ser tomado no processo de redução das imagens é a sua calibração em fluxo. Para efetuá-la, realizamos primeiramente uma fotometria de abertura para o objeto de interesse e para as estrelas padrões disponíveis, apresentadas na Tabela [5.1. Utilizamos uma abertura fixa de 13 píxeis, conforme o recomendado por Verhoeff et al. (2012). Como normalmente não dispomos de estrelas calibradoras suficientes para bem determinar a extinção atmosférica, optamos por utilizar o modelo atmosférico calculado pelo código ATRAN (Lord, 1992), de acordo com o procedimento adotado por exemplo por Fujiwara et al. (2006). A expressão que relaciona o fluxo com a taxa de contagens medida e a massa de ar é dada pela expressão:

$$
F_{\text {source }}=\frac{F_{\text {std }}}{\dot{N}_{\text {std }}} \times \dot{N}_{\text {source }} \times \exp \left[\tau\left(A M_{\text {source }}-A M_{\text {std }}\right)\right]
$$

Na equação, $\dot{N}$ representa a taxa de contagens por unidade de tempo, $\tau$ a profundidade óptica atmosférica e $A M$ a massa de ar de cada observação. Para o caso de mais de uma estrela padrão, o desvio padrão entre os fluxos estimados com base em cada uma delas serve como uma estimativa de erro na determinação do fluxo. A partir deste fluxo obtido, é simples calcularmos o fator de conversão entre contagens e Jy/arcsec ${ }^{2}$, uma unidade frequentemente adotada em trabalhos correlatos na literatura. 
Tabela 5.1 - Dados das estrelas padrões utilizadas.

\begin{tabular}{clllcc}
\hline \hline banda & estrela padrão & $\begin{array}{l}\text { Ascensão Reta } \\
\text { (h:min:s) }\end{array}$ & $\begin{array}{l}\text { Declinação } \\
\text { (grau:min:s) }\end{array}$ & $\begin{array}{c}F_{\text {banda }} \\
(\mathrm{Jy})\end{array}$ & Tipo espectral \\
\hline \multirow{2}{*}{ PAH1 } & HD 50310 & 064956.16846 & -503652.4437 & 42.18 & K1III \\
& HD 151680 & 165009.81081 & -341735.6337 & 68.15 & K2I \\
& HD 152880 & 165640.7861 & -155637.647 & 7.66 & M0/M1III \\
\hline \multirow{2}{*}{ PAH2 } & HD 50310 & 064956.16846 & -503652.4437 & 26.80 & K1III \\
& HD 101666 116976 & 114143.94791 & -322957.8464 & 7.07 & K5III \\
& HD 50310 & 064956.16846 & -503652.4437 & 9.98 & K1III \\
\multirow{2}{*}{ Q2 } & HD 95578 & 110149.67462 & -022904.5007 & 6.50 & M0III \\
\hline \hline
\end{tabular}

O último passo executado no processo de redução dos dados VISIR é a sua calibração astrométrica. No entanto, temos que as imagens finais possuem um tamanho angular tipicamente da ordem de $5^{\prime \prime}$ e a incerteza no apontamento do VLT é de alguns segundos de arco. Portanto, uma calibração absoluta não pode ser feita de maneira autoconsistente. Desta forma, adotamos coordenadas relativas ao centro da imagem, dadas em segundos de arco ou unidades astronômicas.

\subsection{Análise das imagens}

Conforme já mencionado em 5.1, elegemos o objeto PDS340 como o protótipo para o desenvolvimento de nossas ferramentas de redução e análise. Foram selecionadas observações em três bandas para este objeto: PAH1 $(8.59 \mu \mathrm{m})$, PAH2 $(11.25 \mu \mathrm{m})$ e Q2 $(18.72 \mu \mathrm{m})$. Para cada um destes casos foi aplicado o processo de redução descrito na seção anterior. O resultado final deste processo pode ser contemplado na Figura 5.11.

Os contornos das isofotas foram sobrepostos às imagens, de modo a enfatizar os eventuais desvios da simetria circular destas observações. Os fluxos calculados para PDS340 foram (25.0 \pm 0.9) Jy na banda PAH1, (77 \pm 8) Jy na banda PAH2 e (206 \pm 132$)$ Jy na banda Q2. Os erros associados foram estimados a partir do desvio padrão da medida de fluxo baseada em cada estrela padrão. A Figura 5.12 exibe estes valores ao lado de diferentes medidas catalogadas por outras missões em comprimentos de onda na mesma região 


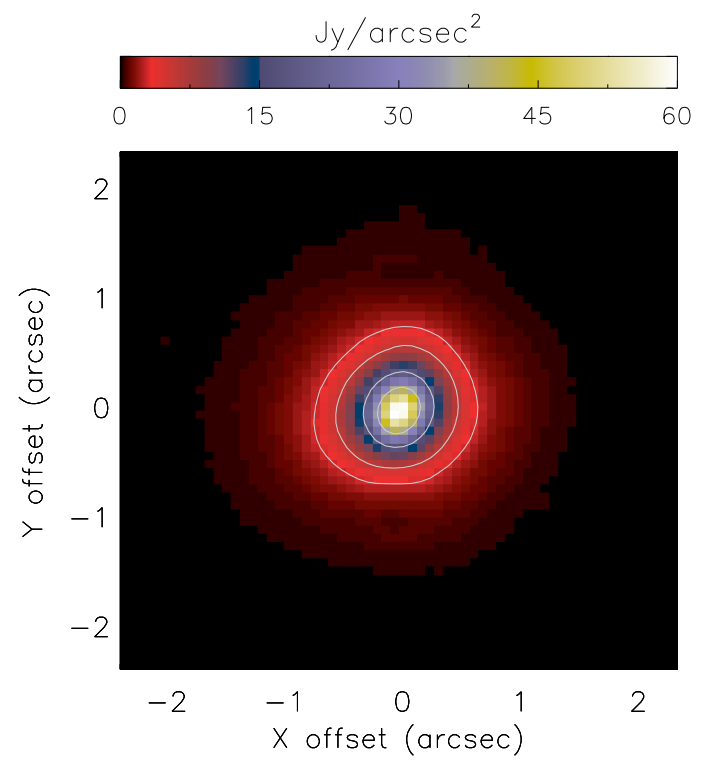

(a) PDS340 na banda PAH1 $(8.59 \mu \mathrm{m})$.

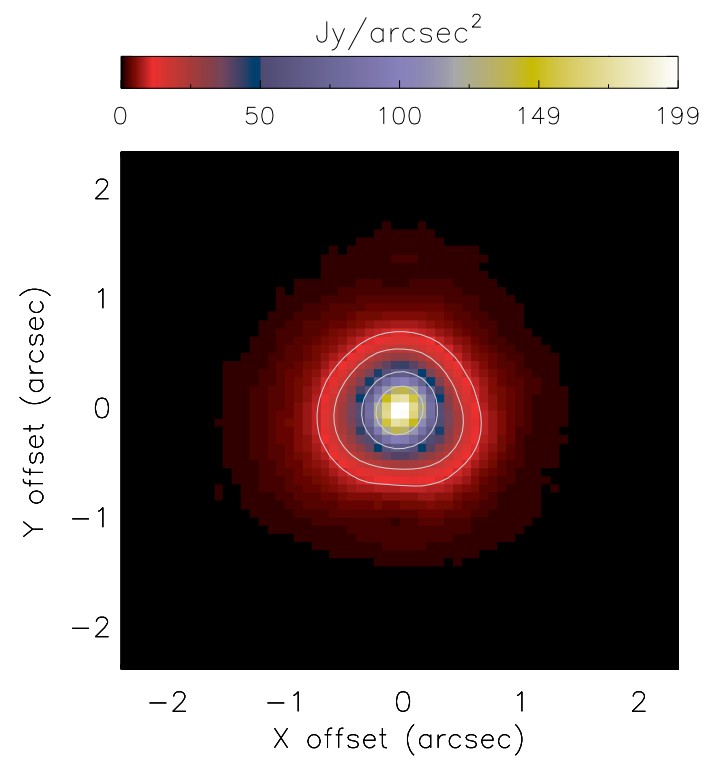

(b) PDS340 na banda PAH2 $(11.25 \mu \mathrm{m})$.

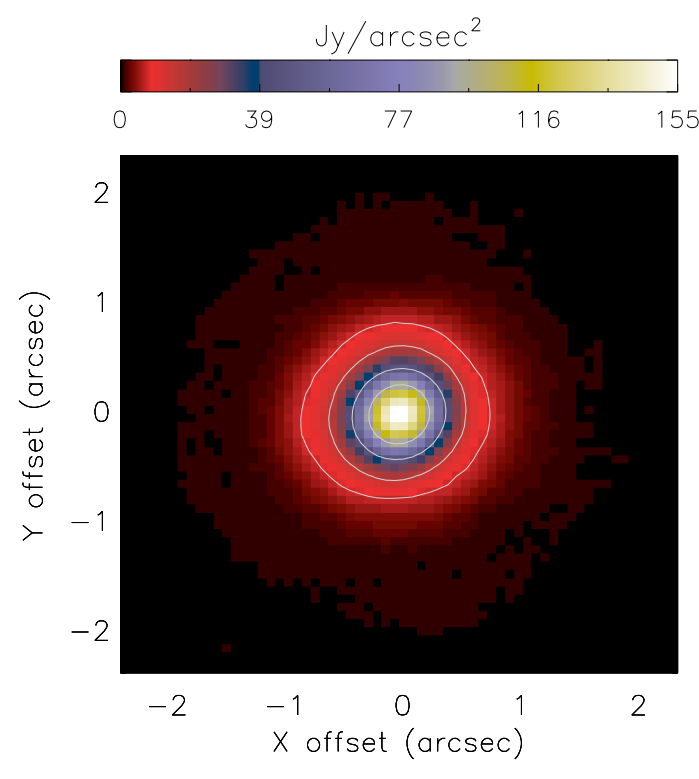

(c) PDS340 na banda Q2 $(18.72 \mu \mathrm{m})$.

Figura 5.11: Imagens corrigidas, combinadas e calibradas em fluxo de PDS340, em três bandas do VISIR no VLT. Sobrepostos às imagens, encontram-se os contornos de mesmo fluxo em 5\%, 10\%, 30\% e 60\% dos seus valores de pico. 


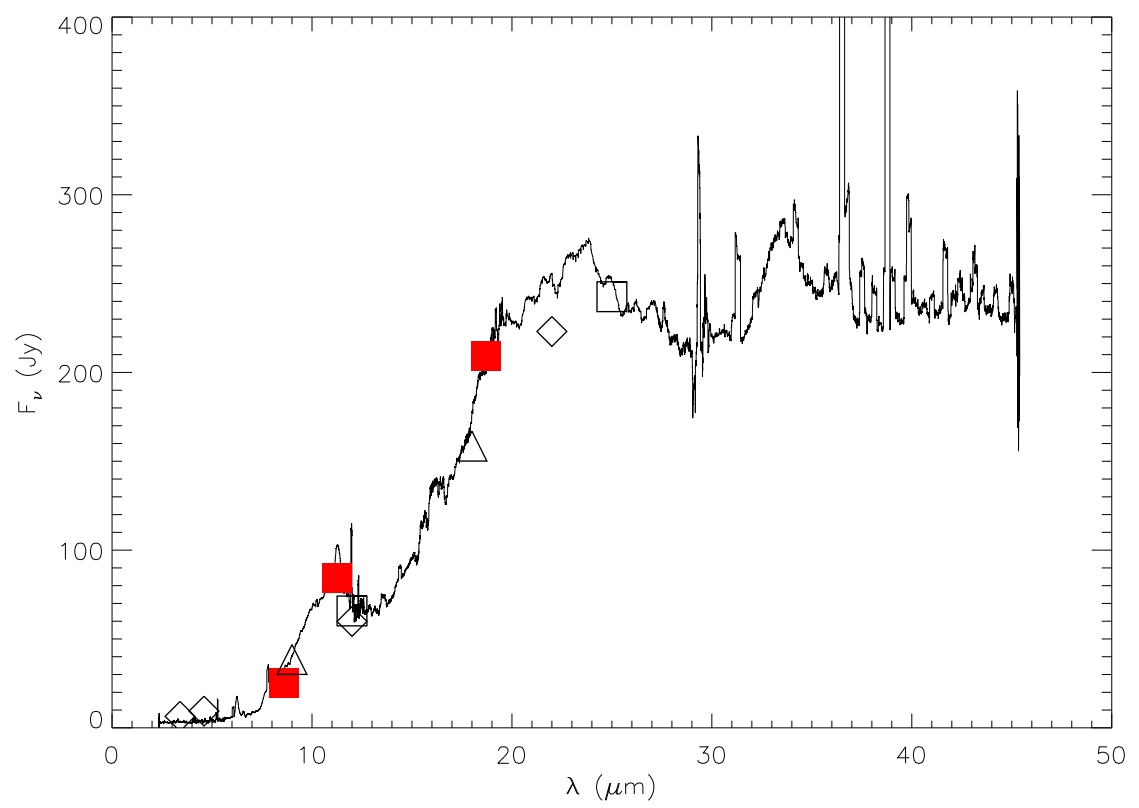

Figura 5.12: Fluxos calculados, confrontados com outras observações feitas em comprimentos de onda próximos. Os quadrados vermelhos representam os fluxos calculados, a curva contínua o espectro ISO SWS, os diamantes as medidas WISE, os triângulos os fluxo AKARI e os quadrados abertos os fluxos IRAS.

espectral.

\subsubsection{Subtração da componente estelar}

Quando observamos as imagens na Figura 5.11, é difícil julgar se há a contribuição do material circunstelar em seu brilho. Para inspecionar sua presença, seguimos o procedimento utilizado por Fujiwara et al. (2006) e Verhoeff et al. (2011). Neste procedimento, a componente estelar de cada uma das imagens é estimada a partir do perfil de brilho de uma estrela de calibração, devidamente re-escalonada de modo a ter o mesmo valor de pico da imagem de PDS340. Esta componente então é subtraída da imagem sob análise, sendo o resíduo um indicativo da contribuição da componente circunstelar.

Para tanto, calculamos para cada banda a média dos perfis de brilho das estrelas padrões disponíveis. O processo de redução por nós desenvolvido garante que o centróide do objeto encontra-se exatamente no seu centro da imagem. Aplicamos então o fator de escala para que a componente estelar estimada tenha o mesmo valor de pico da imagem de PDS340 
e efetuamos a subtração. As Figuras 5.13 e 5.14 exibem os resultados desta operação e o corte vertical central do perfil de brilho em cada banda.

\subsubsection{Deconvolução com a PSF}

A informação espacial disponível nestas imagens pode ainda ser refinada pelo uso do processo de deconvolução com a PSF (Point Spread Function). A PSF pode ser estimada a partir das estrelas padrões disponíveis, combinadas e normalizadas de modo que a integral de brilho na imagem seja igual à unidade. Idealmente, ela representa a resposta dada pelo sistema de observação a uma fonte luminosa puntual. O seu perfil é determinado não só pela turbulência atmosférica, mas também pela resposta instrumental. O efeito da transformação que a imagem "real" sofre durante o processo de observação é quantificado pela expressão:

$I(x, y)=\int_{x^{\prime}=-\infty}^{\infty} \int_{y^{\prime}=-\infty}^{\infty} P\left(x-x^{\prime}, y-y^{\prime}\right) O\left(x^{\prime}, y^{\prime}\right) d x^{\prime} d y^{\prime}+N(x, y)=(P * O)(x, y)+N(x, y)$.

Nesta fórmula, a imagem $I(x, y)$ é resultado da convolução da imagem "real" $O(x, y)$ com o perfil da $P S F$, somada ao ruído $N(x, y)$ associado ao processo de medida. Vemos então que, se não fosse por esta parcela indesejada, o processo de deconvolução se resumiria a uma simples divisão no espaço de Fourier. No entanto, a presença do ruído exige o desenvolvimento de métodos mais elaborados, de forma que o efeito da convolução atmosférico/instrumental do sinal seja minimizado.

A ferramenta adotada para este propósito nesta tese foi o método iterativo de Máxima Verossimilhança (Maximum Likelihood - Richardson 1972; Lucy 1974). Foi utilizada sua implementação escrita em IDL e disponibilizada na biblioteca astronômica da NASA 2 .

\footnotetext{
${ }^{2}$ http://idlastro.gsfc.nasa.gov/contents.html
} 


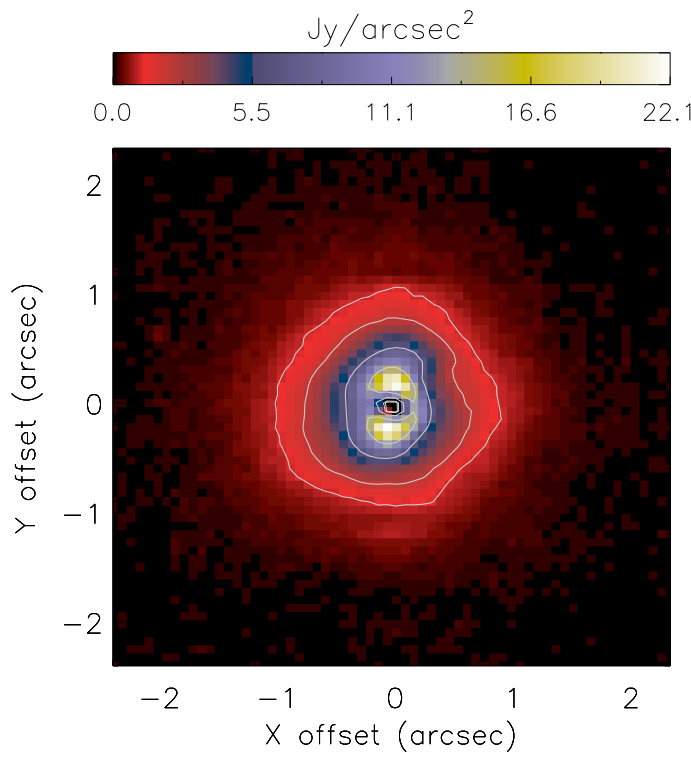

(a) Resíduo em PAH1.

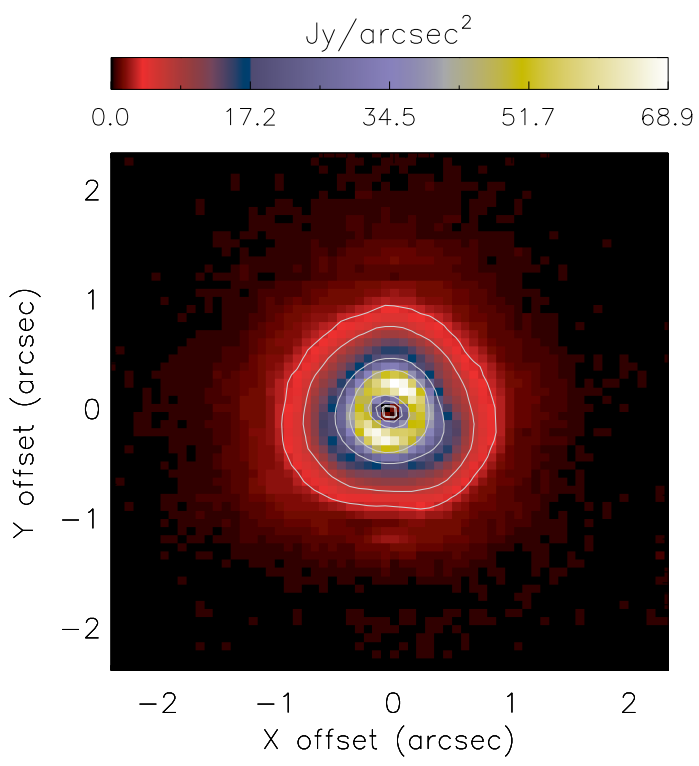

(c) Resíduo em PAH2.

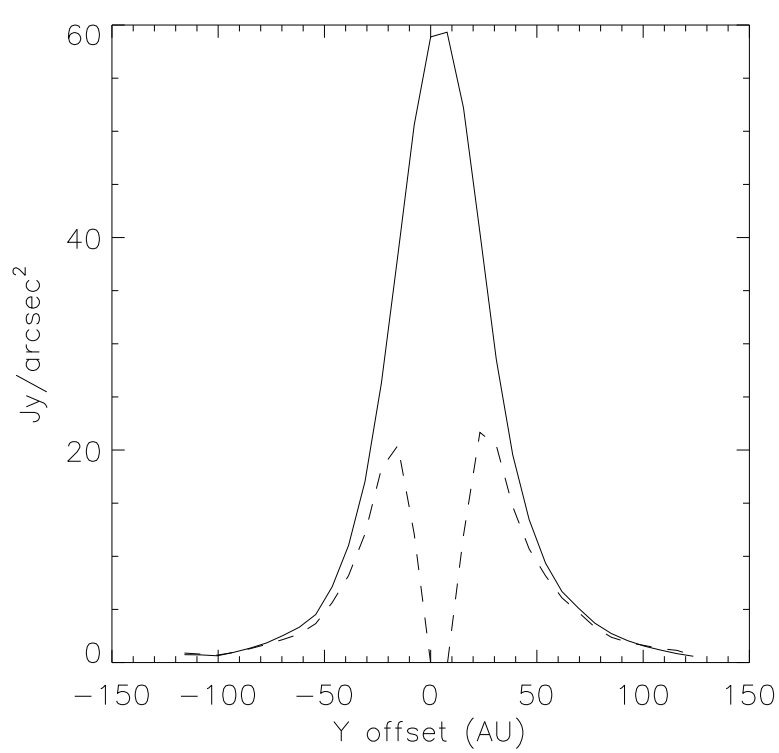

(b) Perfil de brilho em PAH1.

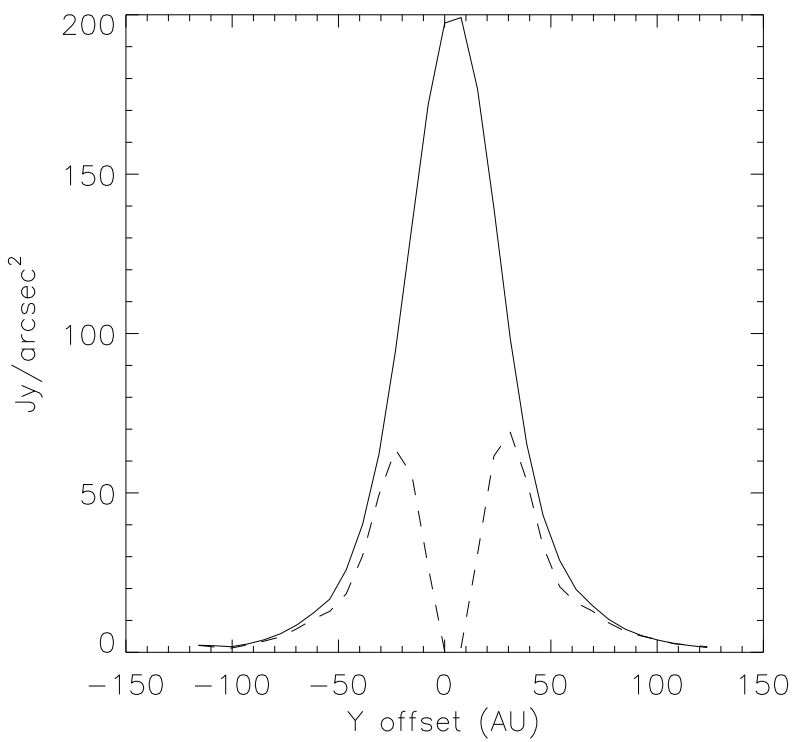

(d) Perfil de brilho em PAH2.

Figura 5.13: Os paineis à esquerda exibem os resíduos obtidos pela subtração da componente estelar da imagem de PDS340 observada nas bandas VISIR. Esta componente foi estimada a partir das estrelas de calibração em cada banda. Sobrepostos às imagens encontram-se os contornos de mesmo fluxo em 5\%, $10 \%, 30 \%$ e $60 \%$ dos seus valores de pico. Os paineis à direita mostram os cortes de perfis verticais de brilho de PDS340, nas respectivas bandas VISIR. A linha cheia representa o perfil de brilho total, enquanto que a linha tracejada representa o perfil de brilho do resíduo obtido através da subtração da componente estelar. 


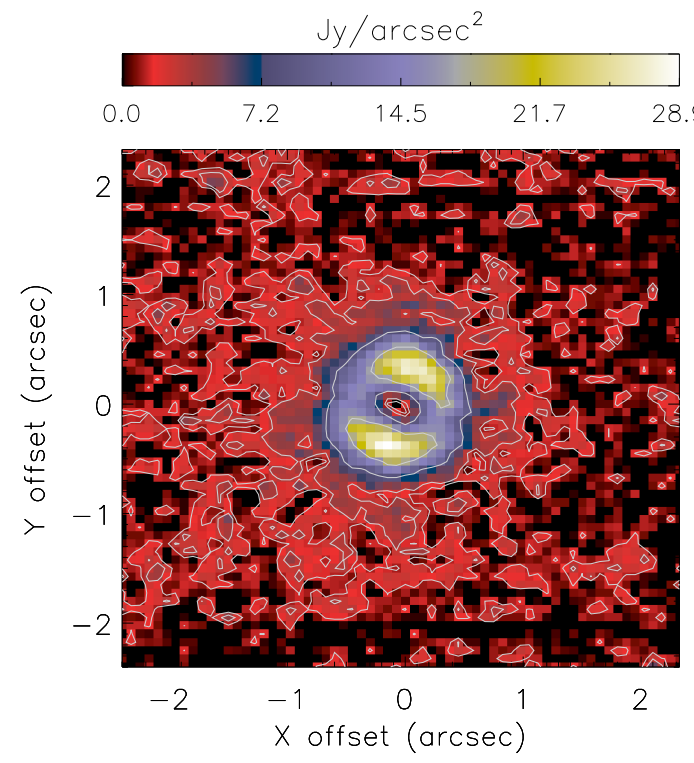

(a) Resíduo em Q2.

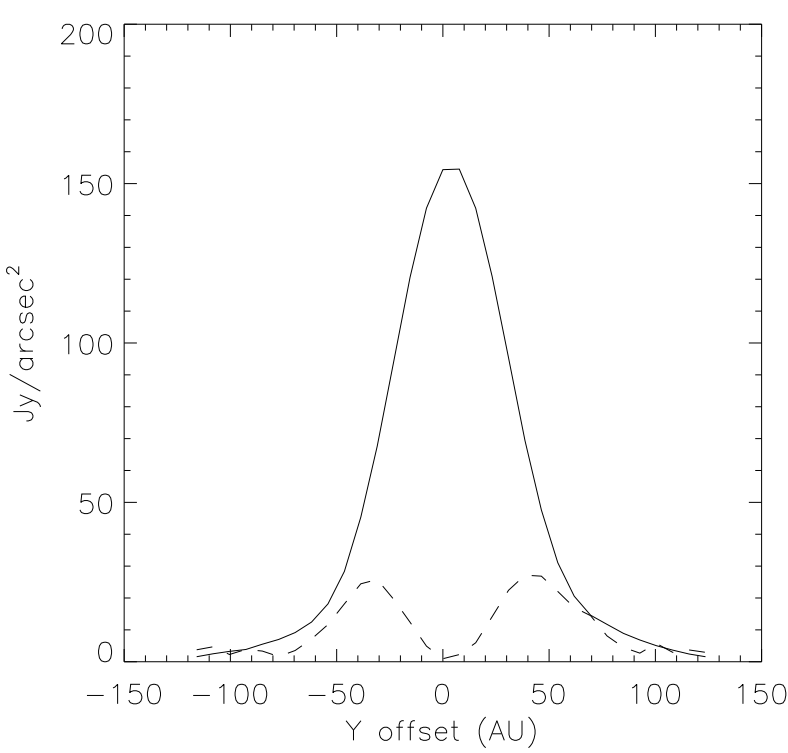

(b) Perfil de brilho em Q2.

Figura 5.14: Continuação da Figura 5.13.

No nosso caso específico, devemos nos preocupar especialmente com as bandas PAH1 $(8.59 \mu \mathrm{m})$ e PAH2 $(11.25 \mu \mathrm{m})$. A solução adotada para a estimativa da $P S F$ foi de apenas considerar as estrelas padrões com medida de seeing menor do que o limite de $0.8^{\prime \prime}$. Esta medida está contida no cabeçalho de todas as observações, onde se discrimina seu valor no início e no fim de cada aquisição. PDS340 possui três estrelas calibradoras suficientemente próximas para cada banda. Entre estas observações, apenas uma se mostrou adequada para compor a estimativa da $P S F$ na banda PAH1, enquanto duas das outras se mostraram aptas para a construção da PSF na banda PAH2. Na banda Q2 utilizamos apenas uma das duas estrelas calibradoras disponíveis para a estimativa do perfil da PSF. A outra estrela apresentou um perfil deformado e teve que ser descartada. A mais provável origem deste problema é a oscilação de chopping acoplada ao sistema de acompanhamento do telescópio, que por vezes produz ao alongamento da própria $P S F$ em alguma direção. Não há uma compreensão completa acerca deste efeito, sendo ele aparentemente aleatório e portanto irreprodutível. Sua ocorrência é tão mais frequente quanto maior for a amplitude do ângulo de chopping (Maury, 2006). A soma das estrelas padrões para a estimativa da PSF é dada por: 


$$
Z(x, y)=\sum_{i} \dot{N}_{i}(x, y) \times \exp \left(\tau \times A M_{i}\right)
$$

Na expressão, $\dot{N}_{i}(x, y)$ representa o número de contagens por unidade de tempo de cada objeto, enquanto que o fator exponencial corrige a extinção atmosférica à qual a observação foi sujeita. Um filtro passa-baixas é então aplicado ao valor de $Z(x, y)$, de modo a minimizar seu ruído. A imagem resultante é finalmente normalizada de modo que a soma do valor em todos os píxeis seja igual à unidade. Os resultados da deconvolução em cada banda das imagens de PSD340 são exibidos na Figura 5.15.

Entretanto, devemos neste ponto enfatizar o quão delicada é esta operação quando a efetuamos sobre observações no IR médio. Como já mencionado no início deste capítulo, as observações VISIR são em princípio limitadas apenas pela difração. No entanto, essa situação pode se reverter e o seeing pode se tornar dominante em algumas ocasiões, dependendo das condições atmosféricas no momento da observação (Pantin, 2011). As informações contidas na Figura 5.2 resumem bem esta situação. Na banda $N$ (em torno da banda VISIR PAH1, por exemplo), apenas observações executadas sob um seeing $\leq 0.8^{\prime \prime}$ serão limitadas por difração. Como o valor de seeing decresce com o comprimento de onda, as observações na banda Q2 $(18.72 \mu \mathrm{m})$ do VISIR são rotineiramente limitadas pela difração.

Quando lidamos com métodos de deconvolução, a estimativa da PSF é o ponto chave de uma boa correção. No caso de uma observação limitada por difração, o perfil da PSF é estático, e portanto as estrelas calibradoras serão suficientemente estáveis para que possamos estimá-lo a partir de seu brilho. Já no caso dominado pelo seeing, o perfil de brilho das estrelas padrões é suscetível às flutuações atmosféricas, não oferecendo portanto uma boa estimativa da $P S F$.

A exemplo da análise feita anteriormente, efetuamos a subtração da componente estelar de PDS340, de modo a ressaltar a emissão circunstelar deste objeto. Note que, nestes 


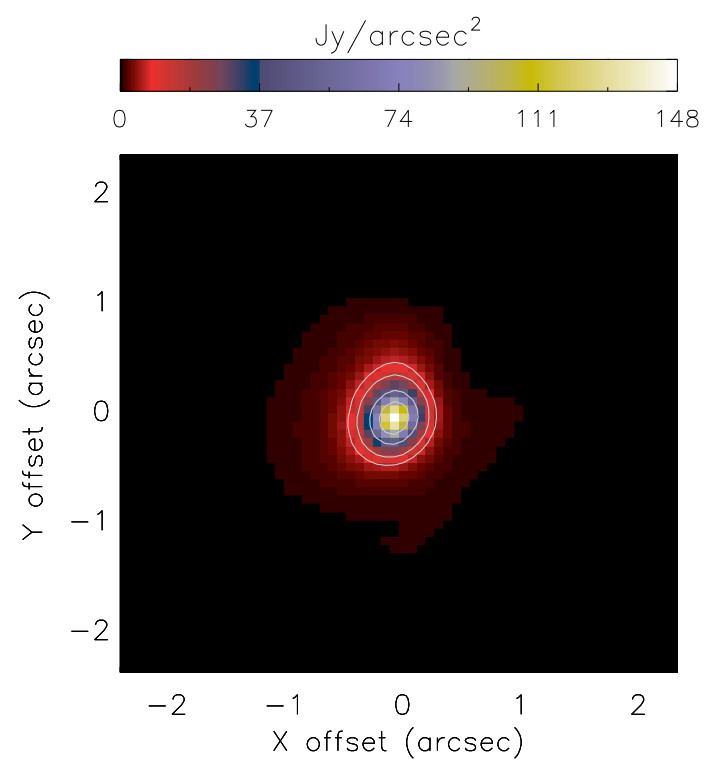

(a) Imagem deconvoluída na banda PAH1.

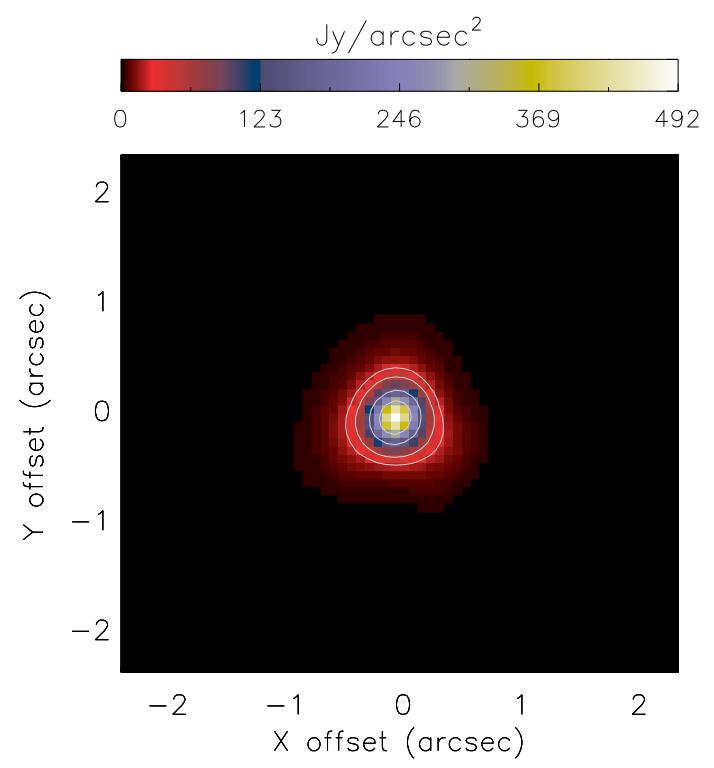

(b) Imagem deconvoluída na banda PAH2.

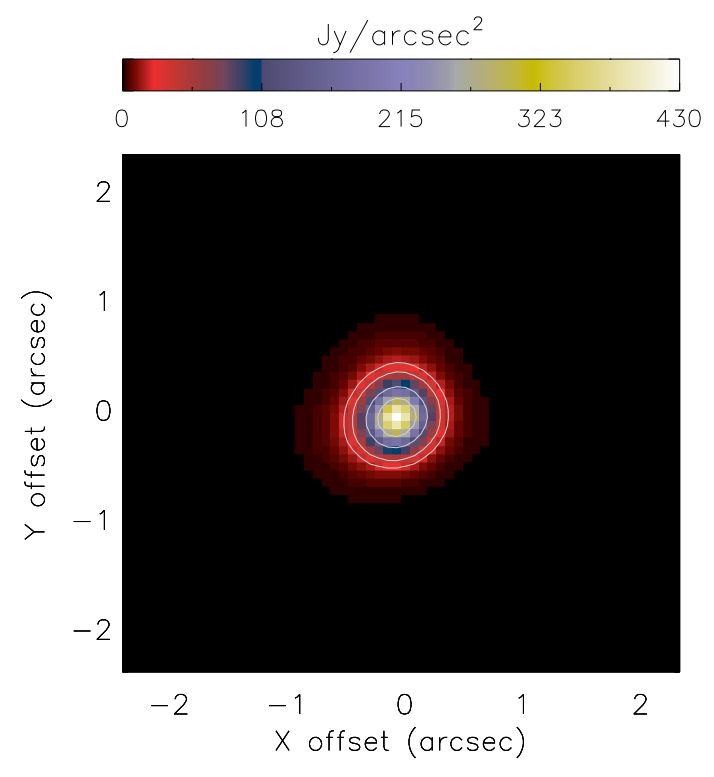

(c) Imagem deconvoluída na banda Q2.

Figura 5.15: Imagens de PDS340 nas bandas VISIR, deconvoluídas com o uso da PSF estimada à partir das estrelas padrões. Sobrepostos às imagens, encontram-se os contornos de mesmo fluxo em $5 \%, 10 \%$, $30 \%$ e $60 \%$ dos seus valores de pico. 
casos, esta componente corresponde ao próprio perfil da $P S F$, a menos de um fator multiplicativo. Assim, primeiro subtraímos a componente estelar (estimada pela $P S F$ ) e depois executamos a deconvolução. Os resíduos resultantes da subtração da componente estelar e seus respectivos perfis verticais de brilho encontram-se nas Figuras 5.16 e 5.17 .

Outra alternativa para eliminação do ruído é o ajuste da $P S F$ obtida anteriormente por um perfil analítico. Para todos os casos, realizamos o ajuste desta distribuição de brilho à função de Moffat:

$$
Z^{\prime}(x, y)=Z_{0}\left[1+\left(\frac{x}{a}\right)^{2}+\left(\frac{y}{b}\right)^{2}\right]^{-\beta}
$$

Esta forma analítica é frequentemente empregada na estimativa de PSFs sintéticas (Starck e Murtagh, 2002). Além da variação dos parâmetros expressos na equação, também permitimos a variação do seu ângulo de orientação. Desta forma, nos deparamos com o empecilho de encontrar o melhor ajuste para uma função de cinco parâmetros. Para resolver este problema, fizemos novamente uso dos algoritmos genéticos, descritos do Capítulo 3 ,

O ajuste se deu por meio da minimização da função $\iint\left[Z(x, y)-Z^{\prime}(x, y)\right]^{2} d x d y$, aplicando-se os já descritos operadores genéticos sobre "gerações" consecutivas de soluções. Um ajuste gaussiano também foi aplicado sobre os perfis da $P S F$, de modo a estabelecermos uma comparação entre ambos os ajustes. No entanto, apesar do perfil de Moffat apresentar melhores resultados do que o ajuste gaussiano, ele não se mostrou satisfatório para nossos propósitos. A diferença entre os perfis observado e sintético não é causada apenas pelo ruído, como esperaríamos no caso ideal (Figura 5.18). Isto significa que a sua utilização no processo de deconvolução implicaria em uma perda não desprezível da informação original fornecida pelo perfil de brilho das estrelas padrões. Portanto, a fim de evitarmos a obtenção de resultados espúrios, optamos por utilizar apenas a combinação das estrelas calibradoras. 


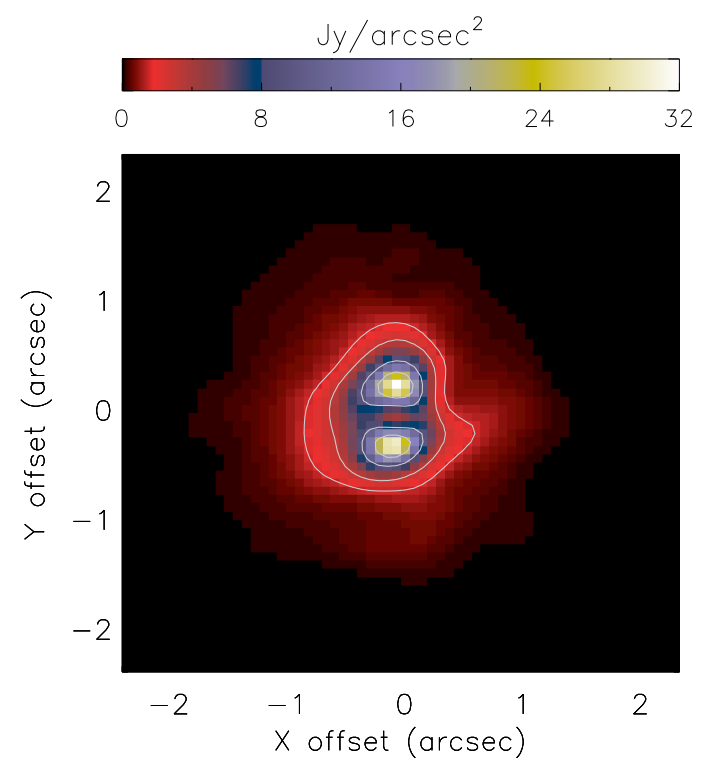

(a) Resíduo deconvoluído na banda PAH1.

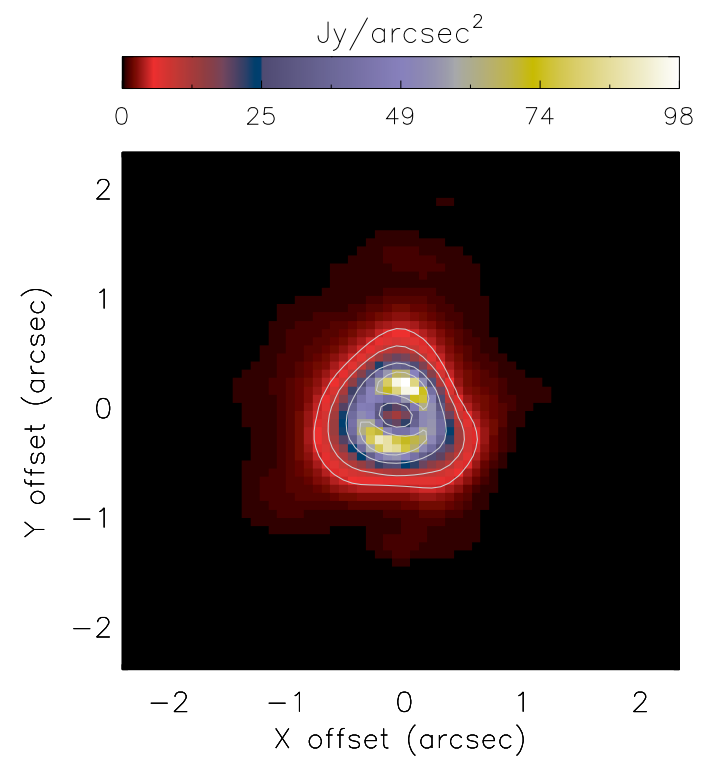

(c) Resíduo deconvoluído na banda PAH2.

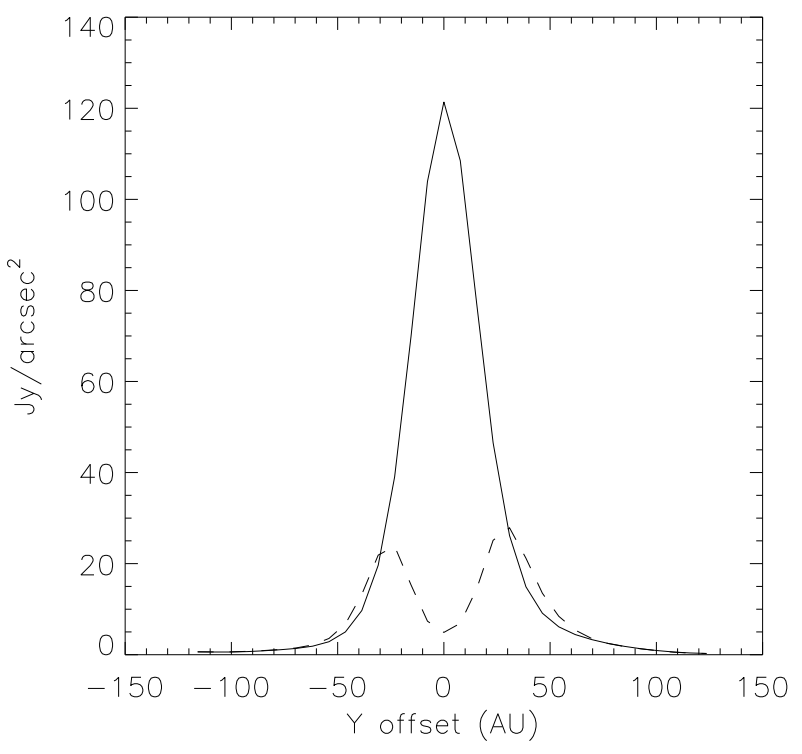

(b) Perfil de brilho em PAH1.

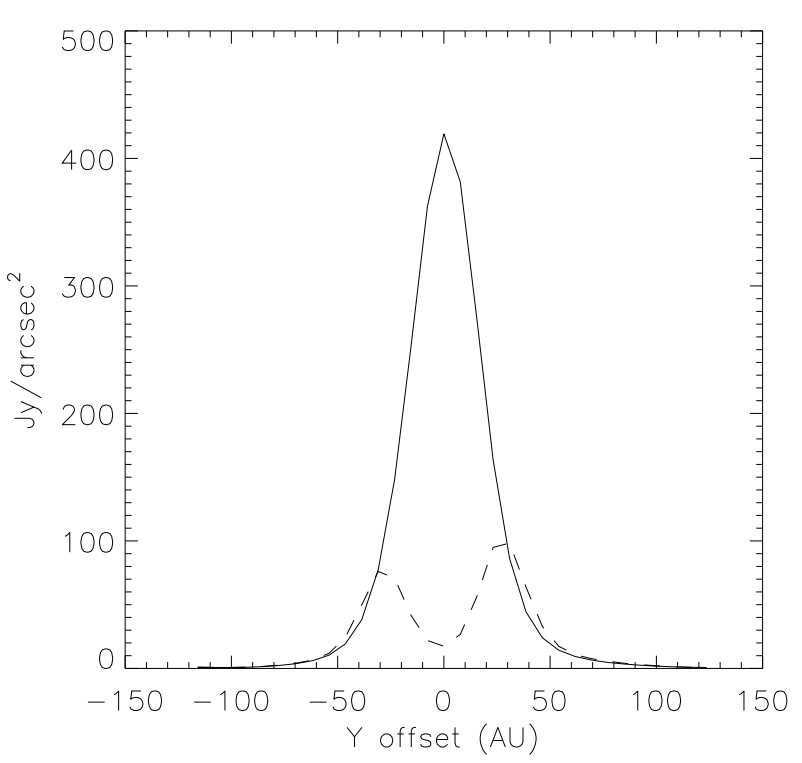

(d) Perfil de brilho em PAH2.

Figura 5.16: À esquerda são apresentadas as imagens residuais de PDS340 nas bandas VISIR, deconvoluídas utilizando-se as PSFs estimadas a partir das estrelas de calibração. Sobrepostos às imagens, encontram-se os contornos de mesmo fluxo em 5\%,10\%,30\% e 60\% dos seus valores de pico. Os paineis à direita mostram os cortes de perfis das respectivas imagens. A linha cheia representa o perfil de brilho total, enquanto que a linha tracejada representa o perfil de brilho do resíduo obtido por meio da subtração da componente estelar. 


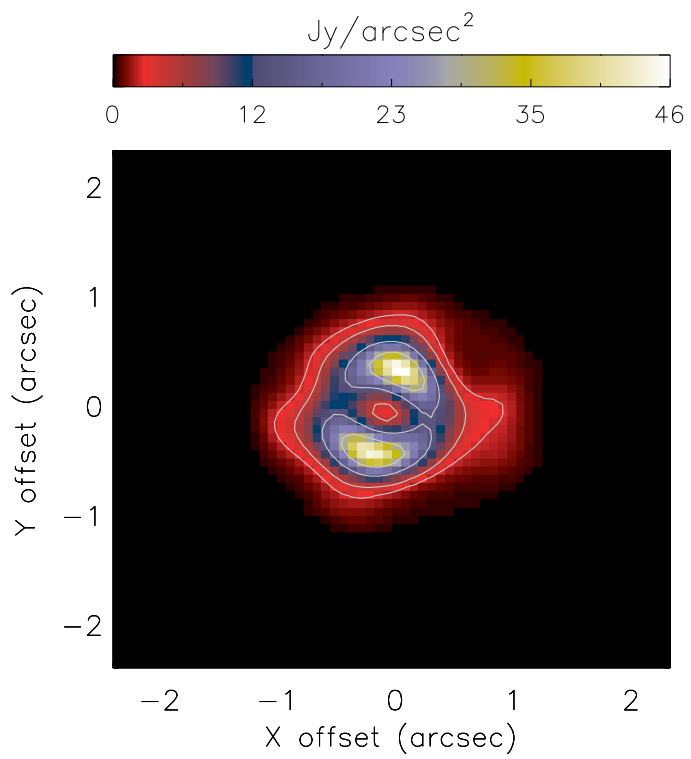

(a) Resíduo deconvoluído na banda Q2.

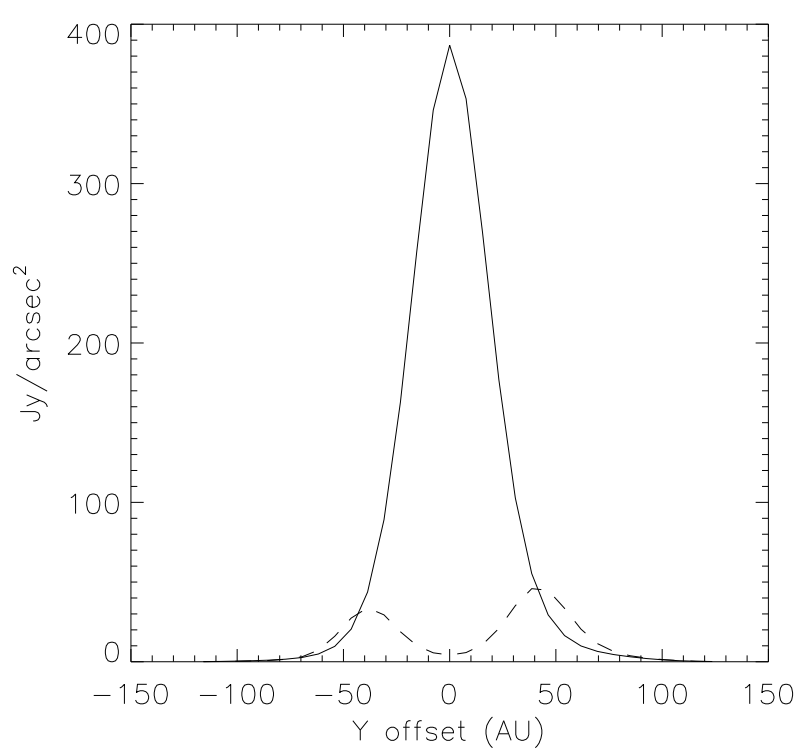

(b) Perfil de brilho em Q2.

Figura 5.17: Continuação da Figura 5.16

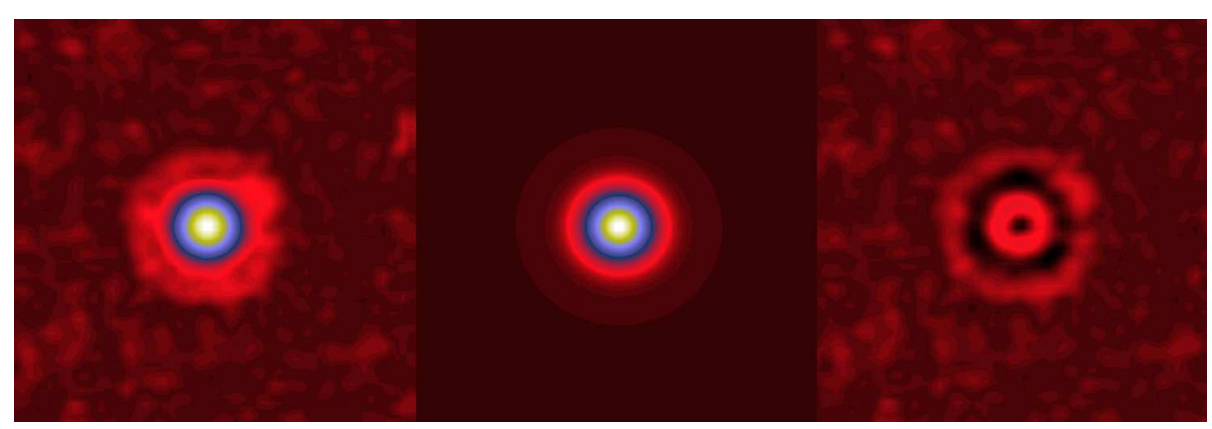

Figura 5.18: Ajuste da PSF através do perfil analítico de Moffat. À esquerda, exibimos a PSF estimada a partir da combinação das estrelas padrões, na banda Q2. Ao centro, temos o perfil analítico deduzido e à direita apresentamos o resíduo do ajuste.

\subsection{Discussão}

A análise das imagens nos leva à constatação da presença de material circunstelar associado ao objeto PDS340. Note-se ainda que este resultado é independente do processo de deconvolução, conforme exibido nas Figuras 5.13 e 5.14. Se tomarmos a largura à meia altura das estrelas padrões como uma estimativa da resolução angular obtida por estas imagens VISIR, temos que $R=0.4^{\prime \prime}(\sim 40 \mathrm{AU})$. Esta é justamente a ordem de grandeza 
das sub-estruturas encontradas nos resíduos da subtração da componente estelar, o que demonstra que sua detecção é suficientemente confiável.

A distribuição alongada desta estrutura sugere que o material observado se encontra sob a forma de um disco, o que está de acordo com a confirmação recente de Goto et al. (2012) para este objeto. O perfil de brilho nos mostra que a contribuição relativa desta estrutura para o brilho total é mais importante nas bandas PAH1 e PAH2 do que para a banda Q2. Além disso, a emissão em $18.72 \mu \mathrm{m}$ é mais extensa do que nas outras bandas.

De forma a extrair parâmetros quantitativos destas observações, realizamos o ajuste de elipses às isofotas à meia altura de cada uma das imagens deconvoluídas da PSF. Os resultados deste ajuste podem ser encontrados na Tabela 5.2. Sendo $a$ e $b$ respectivamente os eixos maior e menor da elipse, sua excentricidade é definida por $\varepsilon=\sqrt{1-(b / a)^{2}}$. A inclinação do sistema é estimada por $i=\cos ^{-1}(b / a)$, adotando-se a simples hipótese de que a estrutura é circular.

Tabela 5.2 - Parâmetros estimados a partir do ajuste elíptico das isofotas à meia altura das imagens de PDS340. O parâmetro a representa o tamanho do semi-eixo maior da elipse, $\varepsilon$ a sua excentricidade, $P A$ o ângulo de posição com respeito ao eixo-x e $i$ a estimativa de inclinação do sistema.

\begin{tabular}{lccccc}
\hline \hline banda & $\lambda(\mu \mathrm{m})$ & $a$ & $\varepsilon$ & $P A$ & $i$ \\
\hline PAH1 & 8.59 & $0.18^{\prime \prime}$ & 0.58 & $75^{\circ}$ & $35.7^{\circ}$ \\
PAH2 & 11.25 & $0.18^{\prime \prime}$ & 0.58 & $75^{\circ}$ & $35.3^{\circ}$ \\
Q2 & 18.72 & $0.21^{\prime \prime}$ & 0.45 & $70^{\circ}$ & $26.8^{\circ}$ \\
\hline \hline
\end{tabular}

As altas excentricidades determinadas quantificam o desvio da simetria circular de PDS340 nestes comprimentos de onda, o que novamente é compatível com a presença de um disco. O ajuste também indica que o eixo deste disco possui um ângulo de cerca de $30^{\circ}$ em relação à direção da linha de visada.

Os perfis de brilho desta estrutura circunstelar apresentam seus máximos de emissão em torno de $30 \mathrm{AU}$ nas bandas PAH1 e PAH2 e $40 \mathrm{AU}$ em Q2. É natural que a emissão seja mais estendida para maiores comprimentos de onda, dado que o material se torna 
cada vez mais frio a medida que nos afastamos da fonte central. Infelizmente, a resolução espacial obtida pelo instrumento VISIR não é suficiente para que tracemos o gradiente de temperatura do disco, pois as distâncias entre os picos de emissão circunstelar nas três bandas é inferior ao limite estimado de $0.4^{\prime \prime}$.

É importante ressaltar que estas dimensões não representam a extensão total do disco, mas sim a região mais próxima do objeto central onde as temperaturas são suficientes para que o material emita entre $8 \mu \mathrm{m}$ e $18 \mu \mathrm{m}$.

Em suma, temos o importante resultado da detecção direta de um disco circunstelar em PDS340. No próximo capítulo, exploraremos as informações aqui discutidas, associadas àquelas fornecidas pela modelagem da distribuição espectral de energia deste objeto. 


\section{Capítulo 6}

\section{Conclusões}

Ao longo desta tese, realizamos o estudo do material circunstelar de estrelas de tipo HAeBe sob vários aspectos. O Capítulo 2 descreveu a confirmação da natureza jovem da amostra PDS, o que constitui o primeiro passo do estudo. O Capítulo 3 apresentou a análise da química da poeira destes ambientes circunstelares, a qual está intimamente relacionada à evolução global do sistema jovem. No Capítulo 4 estudamos o status evolutivo dos objetos mais embebidos de nossa amostra, utilizando o diagrama $L_{b o l} \times M_{e n v}$. Finalmente, no Capítulo 5 analisamos mais profundamente as informações geométricas da estrutura circunstelar de PDS340, contidas nas imagens VISIR de alta resolução espacial.

Sob uma perspectiva mais ampla, podemos dizer que todas estas análises desenvolvidas nos levam a um conjunto de informações complementares. A completa compreensão dos ambientes circunstelares de estrelas jovens exige o conhecimento de sua estrutura, sua química e seu estágio evolutivo na pré-sequência principal (PMS). E portanto, para obtenção de modelos realistas destes objetos, é necessário levar em consideração todos estes ingredientes.

Desta forma, podemos apontar a interconexão entre as várias análises apresentadas durante este trabalho. A determinação da natureza dos objetos de PMS é obtida por propriedades de seu material circunstelar. Os diagramas de cores e estimativas da extinção circunstelar discutidos no Capítulo 2 nada mais são do que diagnósticos da distribuição deste material ao redor das estrelas. A química dos grãos de poeira se mostrou relacionada não apenas ao seu ambiente, mas também ao estágio evolutivo do objeto jovem. 
A transição do perfil do silicato de absorção para emissão, o crescimento do grãos e sua cristalização são indícios da evolução global do material circunstelar. Esta evolução, por sua vez, pôde também ser analisada por meio da estimativa da massa do envoltório dos objetos jovens, que se depositam sobre a estrutura de discos e/ou são dissipadas ao longo da história destas estrelas. Finalmente, a análise da morfologia do material circunstelar por meio do imageamento no infravermelho (IR) médio permite a compreensão do ambiente onde se origina a emissão/absorção do silicato, além de revelar características da estrutura circunstelar responsável pela distribuição de energia observada.

Modelos de SED conseguem abranger a maioria das informações relevantes para o estudo do material circunstelar. No entanto, estes modelos dependem em geral de muitos parâmetros, cuja determinação representa um problema nada trivial. Além da dificuldade na obtenção do conjunto de parâmetros mais adequado para um determinado objeto, a unicidade do ajuste também pode ser questionada. Robitaille et al. (2007 - ROB07 daqui em diante) argumentam que seu modelo, dependente de 14 parâmetros, não pretende determinar o valor real de um determinado parâmetro, mas sim o quão bem restrito está cada valor dentro de um certo intervalo. O estreitamento do intervalo de confiança de cada parâmetro, por sua vez, depende da quantidade de dados disponíveis para o objeto sob estudo.

O artigo de Preibisch et al. (2011) mostra uma série de ajustes feitos a partir do modelo de ROB07, com valores próximos de $\chi^{2}$, com conjuntos de parâmetros bastante divergentes. Estes autores argumentam que a ambiguidade do ajuste pode ser eliminada quando também levamos em consideração as informações morfológicas contidas nas imagens. É claro que na prática, dispomos atualmente de uma base de dados fotométrica muito maior do que imagens com alta resolução espacial disponíveis. Grandes missões atuais como o Spitzer (Werner et al., 2004) e o Herschel (Pilbratt, 2008) são fontes de volumes de dados fotométricos gigantescos, que apesar de não disporem de informação espacial detalhada devem ser analisados da melhor forma possível.

Por isso, o conhecimento profundo dos poucos casos onde informações espaciais são 
disponíveis pode levar à construção de modelos de SED mais consistentes, que por sua vez possibilitarão o estudo de grandes amostras.

\subsection{Comparação entre os modelos de SED}

A distribuição de energia de PDS340 (objeto estudado no Capítulo 5) é típica de um objeto jovem em um estágio mais evoluído na PMS. A Figura 6.1 exibe as fotometrias e os espectros ISO disponíveis para este objeto. Sobreposto às observações, incluímos o modelo fotosférico de Castelli e Kurucz (2004) de parâmetros $T_{\text {eff }}=10500 \mathrm{~K}$ e $\log g=4.5$. Uma lei de extinção foi aplicada a este modelo, com $A_{V}=0.21$ extraído dos mapas de Dobashi et al. (2005).

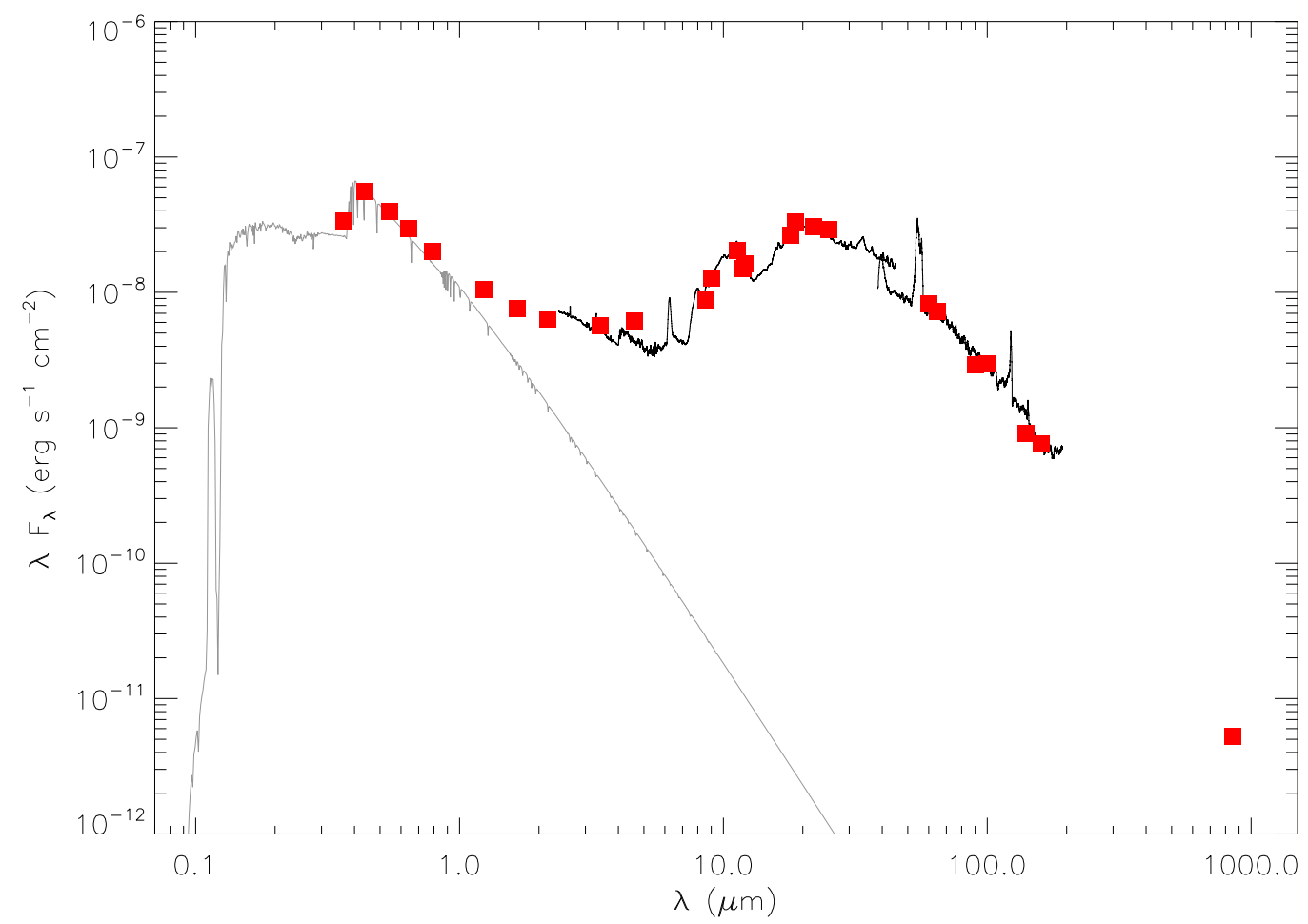

Figura 6.1: SED observada para o objeto PDS340. Sobreposta às fotometrias, a linha escura representa os espectros ISO SWS e LWS. A linha clara representa o modelo fotosférico de Castelli \& Kurucz (2004), para $T_{e f f}=10500 \mathrm{~K}$ e $\log g=4.5$. Foi aplicada a este modelo uma lei de extinção compatível com $A_{V}=0.21$. 
Utilizamos a grade de modelos disponibilizada por ROB07 para o ajuste desta SED. A Figura 6.2 exibe os dez melhores ajustes (i.e., de menor $\chi^{2}$ ) para as fotometrias observadas. Em destaque (linha mais espessa), temos o ajuste de menor $\chi^{2}$ e suas componentes estelar e de disco. Os parâmetros indicados por estes dez modelos podem ser encontrados na Tabela 6.1. Nenhuma destas soluções indicou a presença de uma componente de envoltório neste objeto.

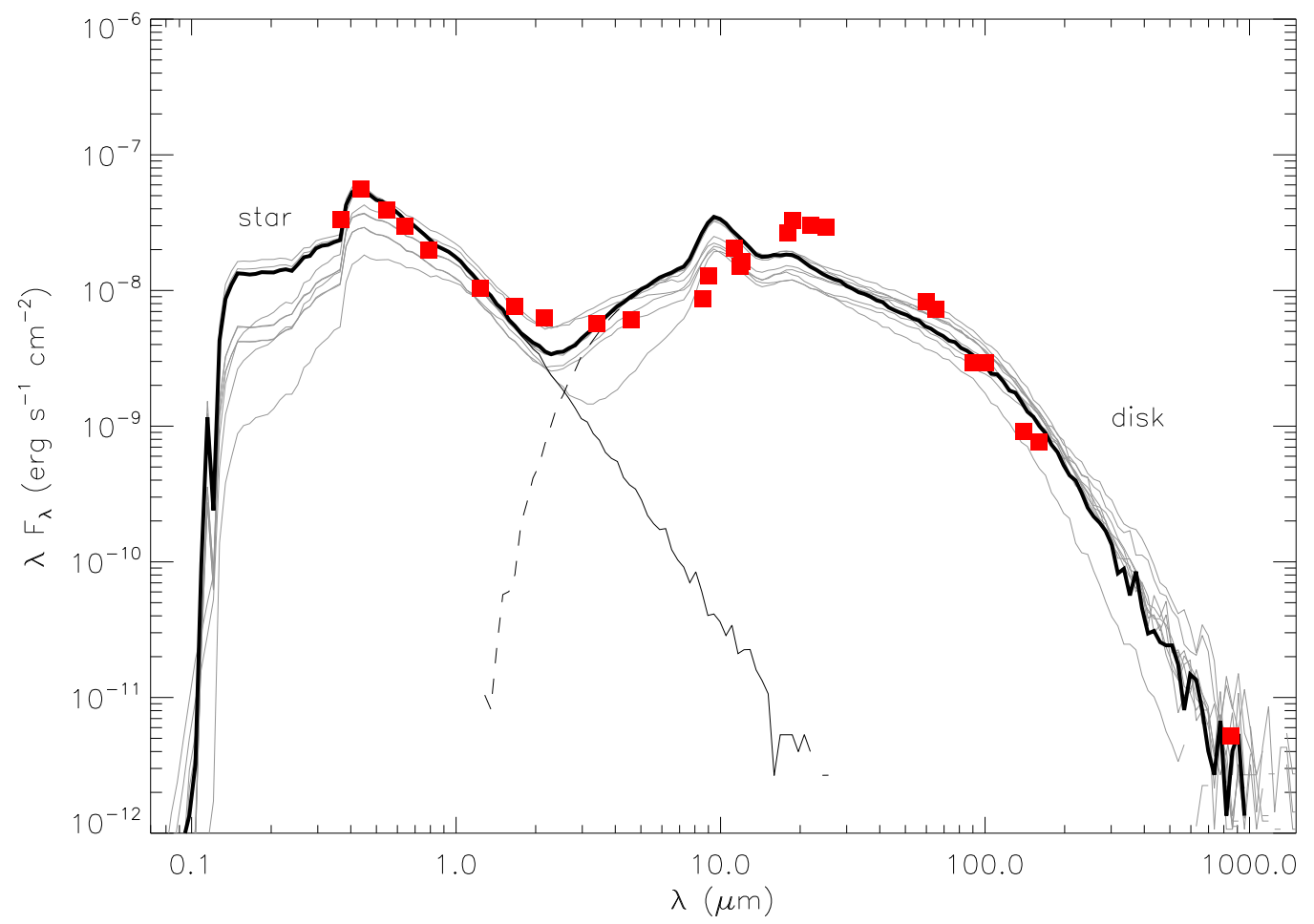

Figura 6.2: Dez melhores ajustes de SED para PDS340, com o modelo de Robitaille et al. (2007). A linha mais escura destaca a solução de menor $\chi^{2}$. As fotometrias observadas são sobrepostas aos ajustes.

A Tabela 6.1 mostra que as melhores soluções encontradas pelo ajuste de ROB07 indicam uma grande variedade de inclinações para o sistema. A análise das imagens VISIR de PDS340 no Capítulo 5 nos levou a um valor de inclinação de $\sim 35^{\circ}$, o qual é bastante próximo do valor determinado por Ardila et al. (2007) por meio de observações com o HST. O conhecimento prévio desta grandeza poderia portanto eliminar muitas das soluções encontradas. Infelizmente, a ferramenta online disponibilizada por ROB07 não permite a 
Tabela 6.1 - Parâmetros indicados pelas dez soluções de menor $\chi^{2}$, obtidas à partir do ajuste dado pelo modelo de ROB07. Entre os parâmetros apresentados, $M_{d}$ representa a massa do disco, $R_{\text {in }}$ e $R_{\text {out }}$ indicam os raios interno e externo do disco, e $H_{100}$ representa a escala de altura do disco em um raio de $100 \mathrm{AU}$.

\begin{tabular}{ccccccc}
\hline \hline$\chi^{2}$ & $\begin{array}{c}\text { Inclinação } \\
\text { graus }\end{array}$ & $\begin{array}{c}L_{\text {bol }} \\
\left(L_{\odot}\right)\end{array}$ & $\begin{array}{c}M_{d} \\
\left(M_{\odot}\right)\end{array}$ & $R_{\text {in }}$ & $\begin{array}{c}R_{\text {out }} \\
(\mathrm{AU})\end{array}$ & $H_{100}$ \\
\hline 388.45 & 69.5 & 42.0 & 0.031 & 15.1 & 196.4 & 7.5 \\
390.75 & 63.3 & 42.0 & 0.031 & 15.1 & 196.4 & 7.5 \\
447.2 & 41.4 & 42.0 & 0.031 & 15.1 & 196.4 & 7.5 \\
580.36 & 75.5 & 44.1 & 0.017 & 26.7 & 454.1 & 6.2 \\
594.58 & 56.6 & 42.0 & 0.031 & 15.1 & 196.4 & 7.5 \\
606.26 & 49.5 & 42.0 & 0.031 & 15.1 & 196.4 & 7.5 \\
611.34 & 31.8 & 42.0 & 0.031 & 15.1 & 196.4 & 7.5 \\
627.75 & 31.8 & 44.7 & 0.047 & 10.0 & 110.8 & 5.6 \\
632.73 & 18.2 & 44.7 & 0.047 & 10.0 & 110.8 & 5.6 \\
636.54 & 18.2 & 42.0 & 0.031 & 15.1 & 196.4 & 7.5 \\
\hline \hline
\end{tabular}

adição de dados morfológicos em seus ajustes, o que como vimos pode indicar descrições equivocadas dos objetos jovens.

Além disto, podemos notar na Figura 6.2 que os fluxos nas bandas 2MASS não são satisfatoriamente ajustados pelo modelo de ROB07. A região do disco mais próxima da estrela $(r \leq 1 \mathrm{AU})$ é provavelmente responsável por este excesso. No entanto, a física desta porção interna ainda é pouco conhecida, devido à dificuldade de resolvê-la espacialmente.

Para reproduzir este excesso no IR próximo, Dullemond et al. (2001) incluem uma borda interna mais espessa ao modelo de disco alargado de Chiang e Goldreich (1997). Estes autores argumentam que o aumento na escala de altura desta região é devido à sua direta exposição ao fluxo estelar. Esta fonte de energia torna a borda interna muito mais quente do que o restante do disco, e consegue explicar o excesso entre $2 \mu \mathrm{m}$ e $3 \mu \mathrm{m}$.

Hetem e Gregorio-Hetem (2007) implementaram o método de algoritmos genéticos para o ajuste do modelo proposto por Dullemond et al. (2001), apresentando em seu trabalho alguns resultados para objetos HAeBe detectados pelo PDS. Utilizando este código, obtivemos o ajuste da SED de PDS340 exibido na Figura 6.3. A figura exibe as várias 
componentes propostas pelo modelo: (i) a fonte central; (ii) a borda interna diretamente exposta à radiação estelar; (iii) a camada interior do disco (i.e., de menor escala de altura); e (iv) a superfície da região mais externa do disco, irradiada pelo objeto central.

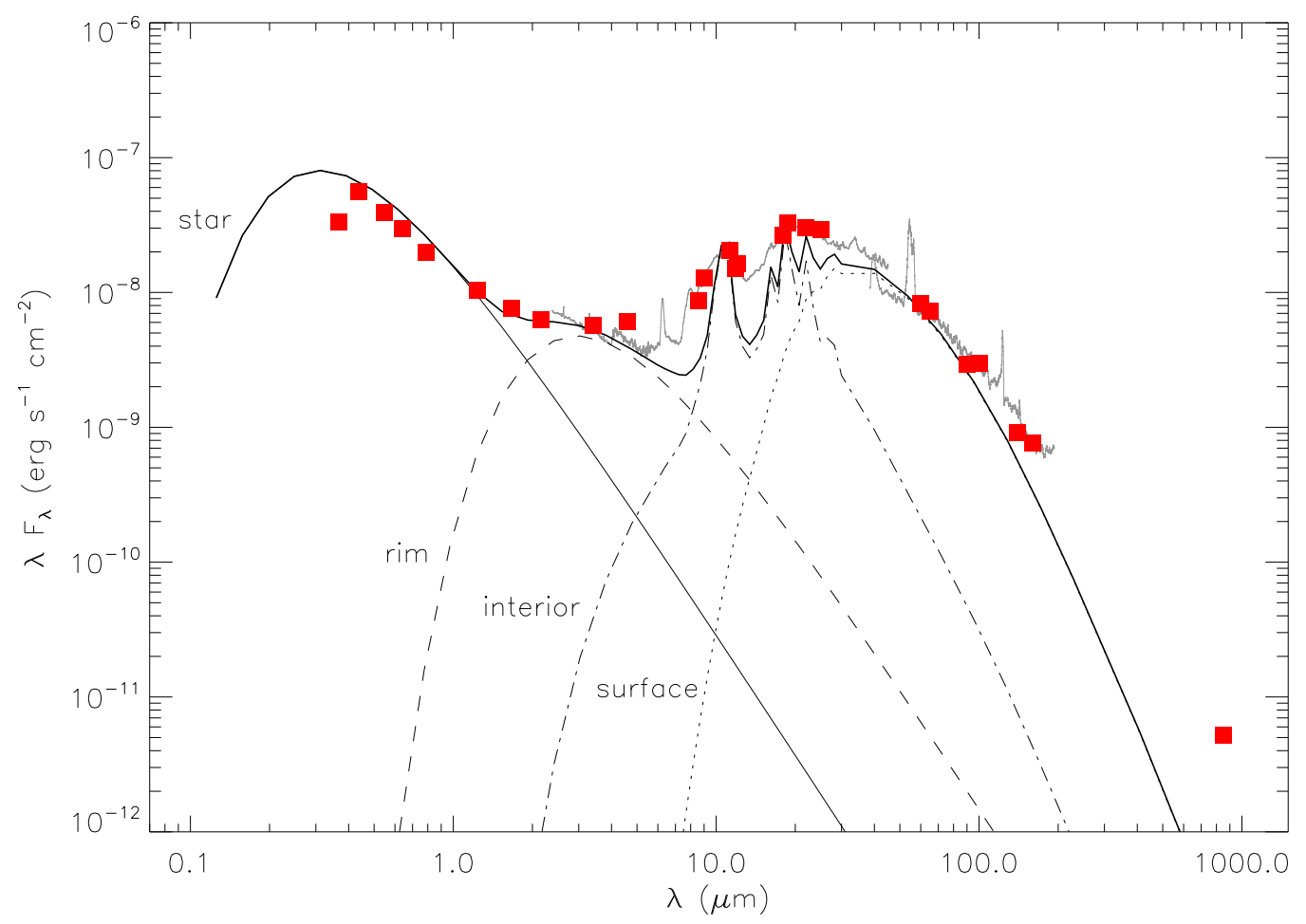

Figura 6.3: Ajuste de SED para PDS340 com modelo de Dullemond et al. (2001), obtido por meio da implementação de algoritmos genéticos (Hetem e Gregorio-Hetem, 2007). A linha cheia mais espessa representa o modelo com todas suas componentes. A contribuição estelar é represantada pela linha cheia menos espessa. A linha tracejada indica a emissão da borda de menor raio do disco, a pontilhada a porção mais interna do disco (em escala de altura). A linha traço-pontilhada indica a contribuição da superfície do disco irradiada pela estrela.

A análise dos parâmetros deste ajuste mostrou que a química da poeira contida no material circunstelar pode desempenhar um papel bastante importante na estrutura do disco. Nossos testes mostraram que a escolha das componentes de poeira utilizadas exerce bastante influência sobre a estimativa da extensão do disco. Se fixamos a massa desta estrutura, grãos com menores valores de opacidade tendem a diminuir o raio do disco e vice-versa. Isto porque o ajuste busca reproduzir uma extinção circunstelar total compatível com as observações, de forma que um material menos opaco deve ser mais denso e 
um material mais opticamente espesso deve ser mais disperso para que possamos manter o valor de $\tau_{V}$ ao longo da linha de visada.

Podemos observar na Figura 6.3 que, embora o espectro em torno do perfil de silicato $(\sim 12 \mu \mathrm{m})$ não ser bem ajustado pelo modelo, a região do IR próximo é bem reproduzida pela borda interna proposta por Dullemond et al. (2001). No entanto, ainda não é claro se esta é a descrição mais adequada do material circunstelar mais próximo da estrela.

A resposta definitiva para esta questão depende do desenvolvimento atual das medidas interferométricas e também da elaboração de modelos mais realistas da região dentro de 1 AU ao redor da estrela. O tratamento adequado da física do material gasoso presente nesta região e a distribuição da poeira na zona de sublimação podem se mostrar decisivos na determinação da estrutura interna.

\subsection{Um modelo completo}

A continuidade natural do presente trabalho é a elaboração de modelos que levem em consideração não apenas o formato da SED, mas também a química da poeira determinada pela espectroscopia no IR médio e as informações morfológicas disponíveis nas observações VISIR (VLT). Conforme já mencionado no Capítulo 5, a base de dados deste instrumento contém imagens e espectros de cerca de vinte objetos HAeBe do PDS. A análise detalhada e conjunta destes dados pode revelar importantes informações sobre o material circunstelar destes objetos.

Ao longo do período de doutorado, tivemos aceitos dois pedidos de tempo Gemini com o instrumento T-ReCS, análogo ao VISIR no VLT. Infelizmente, estas observações foram concedidas em banda 2 e não foram realizadas. Pretendemos desenvolver estas observações em um futuro próximo, de modo a acrescentar novos dados ao nosso estudo.

A química da poeira contida no disco foi determinada no Capítulo 3 em um estudo preliminar com o DUSTY. Na continuidade do trabalho, temos a intenção de determinar as componentes da poeira para todos os objetos que dispõem de medidas espectroscópicas. 
Para tanto, utilizaremos a metodologia descrita por van Boekel et al. (2005), aliada ao ajuste sistemático dos dados pelos algoritmos genéticos.

Finalmente, a coleção de todas as informações analisadas formará a base para modelos mais detalhados. Para a elaboração destes modelos, estamos realizando testes preliminares com o código RADMC-3D 1 desenvolvido por Dullemond. Esta ferramenta realiza o cálculo do transporte radiativo em ambientes de poeira, permitindo geometrias arbitrárias em até três dimensões. Além de reproduzir a SED, o código também produz imagens sintéticas nos comprimentos de onda desejados. As propriedades ópticas das mais diversas componentes de poeira podem ser incluídas de forma flexível nos cálculos realizados.

A Figura 6.4 exibe um ajuste preliminar da SED de PDS340 utilizando o RADMC-3D, feito a partir de um modelo de geometria similar ao de ROB07. A imagem sintética em $18.72 \mu \mathrm{m}$ deste modelo é apresentada na Figura 6.5, onde realizamos a convolução com a imagem da PSF observada de modo a reproduzir as observações.

Nosso objetivo final é ajustar simultaneamente a distribuição de energia e as imagens VISIR disponíveis. Para este fim, pretendemos em princípio produzir uma grade de modelos esparsa, que terá um ajuste fino fornecido pela implementação de algoritmos genéticos. Dado que a química da poeira será previamente determinada pela análise dos espectros no IR médio, o conjunto final de parâmetros do modelo possuirá vínculos consistentes com todas as observações disponíveis.

\footnotetext{
${ }^{1}$ http://www.ita.uni-heidelberg.de/ dullemond/software/radmc-3d/
} 


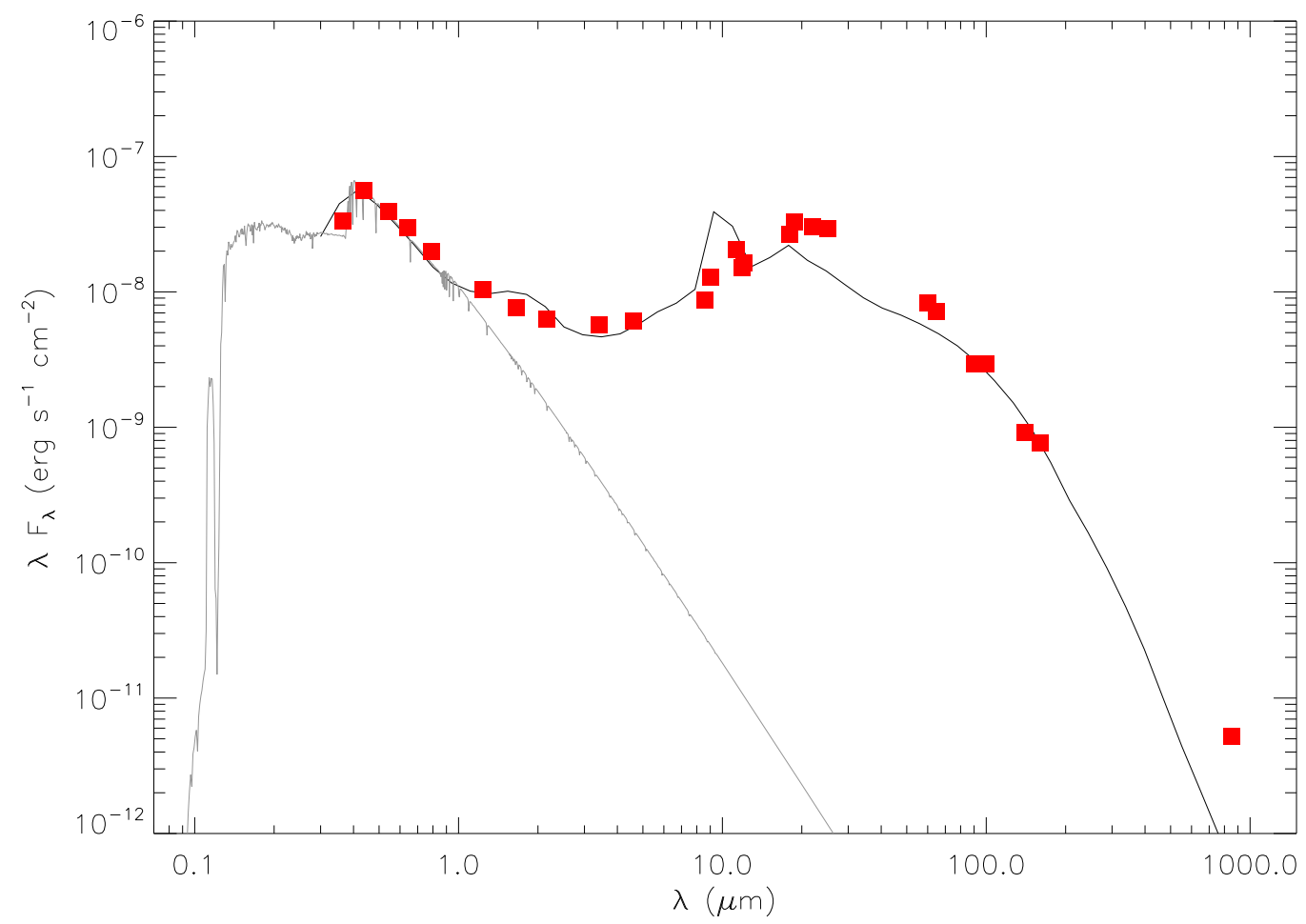

Figura 6.4: Ajuste da SED de PDS340 produzido pelo código RADMC-3D. Sobrepostos, temos as fotometrias disponíveis para este objeto e o modelo fotosférico de Kurucz ( $T_{\text {eff }}=$ $10500 \mathrm{~K}, \log g=4.5)$, utilizado como fonte de energia. 

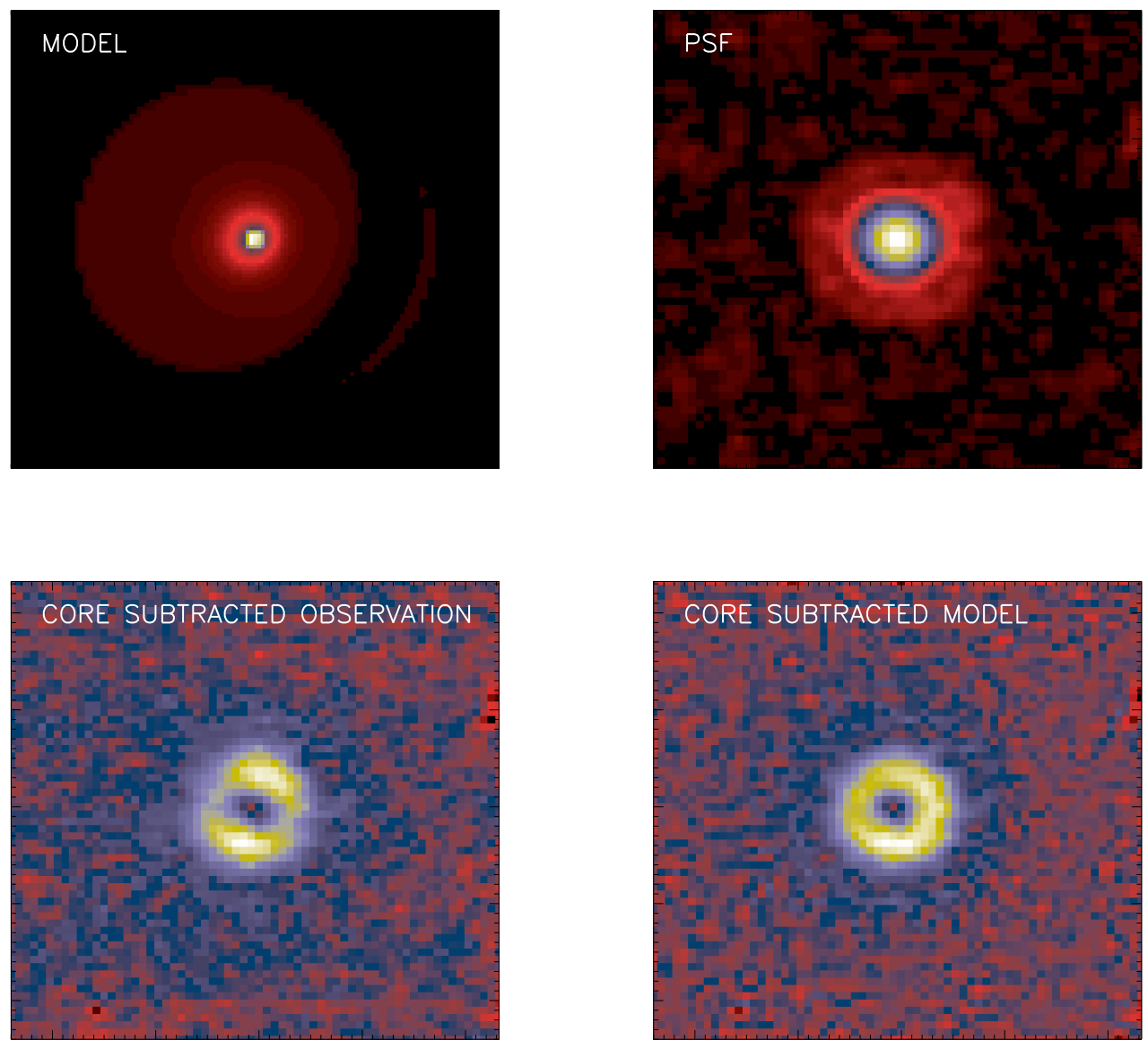

Figura 6.5: O painel superior à esquerda exibe o modelo de disco de PDS340, calculado pelo código RADMC-3D em $18.72 \mu \mathrm{m}$. O painel superior à direita mostra o perfil da PSF observada nesta banda. A imagem inferior à esquerda apresenta a imagem observada de PDS340, com a componente estelar subtraída (veja Capítulo 5). O painel inferior à esquerda exibe o resultado da convolução do modelo com a PSF, também com a componente estelar subtraída. 


\subsection{Perspectivas}

Na sequência deste trabalho, o estudo da composição e estrutura do disco circunstelar será aprofundada. O projeto de pós-doutoramento, sob a supervisão do Prof. Dr. Alex Carciofi, prevê o desenvolvimento de modelos físicos que descrevam o material circunstelar na região mais próxima da estrela.

A motivação fundamental deste estudo é o problema do excesso de emissão no IR próximo, o qual atualmente ainda não é compreendido de maneira satisfatória. Alguns trabalhos apontam como solução para este problema a existência de um bordo mais alargado na região do disco dentro de $1 \mathrm{AU}$ (e.g., Dullemond et al. 2001). No entanto, este vínculo imposto à escala de altura do disco é introduzido de forma artificial, não sendo clara a física destes ambientes. Por isto, é necessário compreendermos esta estrutura mais próxima à estrela a partir de primeiros princípios, levando em consideração todos os efeitos relevantes do problema.

Para tanto, serão desenvolvidos modelos utilizando-se o código HDUST, que calcula o transporte radiativo em meios de gás e poeira. Esta ferramenta permitirá que analisemos não somente a física da borda interna do disco onde há a sublimação da poeira, mas também que levemos em consideração a opacidade do gás contido na região interna a esta borda.

Finalmente, os vínculos adequados aos modelos propostos serão impostos por observações interferométricas no IR próximo/médio e também por medidas espectroscópicas. 


\section{Referências Bibliográficas}

André P., Minier V., Gallais P., Revéret V., Le Pennec J., Rodriguez L., Boulade O., Doumayrou E., Dubreuil D., Lortholary M., Martignac J., First $450 \mu \mathrm{m}$ dust continuum mapping of the massive star-forming region NGC 3576 with the P-ArTéMiS bolometer camera, A\&A, 2008, vol. 490, p. L27

André P., Montmerle T., From T Tauri stars to protostars: Circumstellar material and young stellar objects in the rho Ophiuchi cloud, ApJ, 1994, vol. 420, p. 837

André P., Ward-Thompson D., Barsony M., Submillimeter continuum observations of Rho Ophiuchi A - The candidate protostar VLA 1623 and prestellar clumps, ApJ, 1993, vol. 406 , p. 122

André P., Ward-Thompson D., Barsony M., From Prestellar Cores to Protostars: the Initial Conditions of Star Formation, Protostars and Planets IV, 2000, p. 59

Ardila D. R., Golimowski D. A., Krist J. E., Clampin M., Ford H. C., Illingworth G. D., Hubble Space Telescope Advanced Camera for Surveys Coronagraphic Observations of the Dust Surrounding HD 100546, ApJ, 2007, vol. 665, p. 512

Augereau J. C., Lagrange A. M., Mouillet D., Ménard F., HST/NICMOS2 coronagraphic observations of the circumstellar environment of three old PMS stars: HD 100546, SAO 206462 and MWC 480, A\&A, 2001, vol. 365, p. 78

Beltrán M. T., Cesaroni R., Neri R., Codella C., Furuya R. S., Testi L., Olmi L., Rotating Disks in High-Mass Young Stellar Objects, ApJ, 2004, vol. 601, p. L187 
Beltrán M. T., Cesaroni R., Neri R., Codella C., Furuya R. S., Testi L., Olmi L., A detailed study of the rotating toroids in G31.41+0.31 and G24.78+0.08, A\&A, 2005, vol. 435, p. 901

Benisty M., Renard S., Natta A., Berger J. P., Massi F., Malbet F., Garcia P. J. V., Isella A., Mérand A., Monin J. L., Testi L., Thiébaut E., Vannier M., Weigelt G., A low optical depth region in the inner disk of the Herbig Ae star HR 5999, A\&A, 2011, vol. 531, p. A84

Bessell M. S., Brett J. M., JHKLM photometry - Standard systems, passbands, and intrinsic colors, PASP, 1988, vol. 100, p. 1134

Bjorkman J. E., Circumstellar Disks. In Stellar Atmospheres: Theory and Observations , vol. 497 of Lecture Notes in Physics, Berlin Springer Verlag, 1997, p. 239

Bjorkman J. E., Wood K., Radiative Equilibrium and Temperature Correction in Monte Carlo Radiation Transfer, ApJ, 2001, vol. 554, p. 615

Bontemps S., Andre P., Terebey S., Cabrit S., Evolution of outflow activity around lowmass embedded young stellar objects, A\&A, 1996, vol. 311, p. 858

Bouwman J., Apai D., Chen C., Feigelson E., Grady C., Henning T., Lawson W., Meeus G., Pascucci I., Tielens X., Woodgate B., The evolution of circumstellar disks as traced by coeval stellar clusters: from protoplanetary towards debris disks., Spitzer Proposal, 2005, p. 20691

Bouwman J., Henning T., Hillenbrand L. A., Meyer M. R., Pascucci I., Carpenter J., Hines D., Kim J. S., Silverstone M. D., Hollenbach D., Wolf S., The Formation and Evolution of Planetary Systems: Grain Growth and Chemical Processing of Dust in T Tauri Systems, ApJ, 2008, vol. 683, p. 479

Bouwman J., Meeus G., de Koter A., Hony S., Dominik C., Waters L. B. F. M., Processing of silicate dust grains in Herbig Ae/Be systems, A\&A, 2001, vol. 375, p. 950

Bowers P. F., Knapp G. R., A study of OH/IR stars and planetary nebula formation, ApJ, 1989, vol. 347 , p. 325 
Bowey J. E., Adamson A. J., A mineralogy of extrasolar silicate dust from 10- $\mu$ m spectra, MNRAS, 2002, vol. 334, p. 94

Bringa E. M., Kucheyev S. O., Loeffler M. J., Baragiola R. A., Tielens A. G. G. M., Dai Z. R., Graham G., Bajt S., Bradley J. P., Dukes C. A., Felter T. E., Torres D. F., van Breugel W., Energetic Processing of Interstellar Silicate Grains by Cosmic Rays, ApJ, 2007, vol. 662, p. 372

Castelli F., Kurucz R. L., New Grids of ATLAS9 Model Atmospheres, ArXiv Astrophysics e-prints, 2004

Castilho B. V., Gregorio-Hetem J., Spite F., Barbuy B., Spite M., Detailed analysis of a sample of Li-rich giants, A\&A, 2000, vol. 364, p. 674

Cesaroni R., Olmi L., Walmsley C. M., Churchwell E., Hofner P., A massive young embedded object associated with the UC H II region G31.41+0.31, ApJ, 1994, vol. 435, p. L137

Chiang E. I., Goldreich P., Spectral Energy Distributions of T Tauri Stars with Passive Circumstellar Disks, ApJ, 1997, vol. 490, p. 368

Dobashi K., Uehara H., Kandori R., Sakurai T., Kaiden M., Umemoto T., Sato F., Atlas and Catalog of Dark Clouds Based on Digitized Sky Survey I, PASJ, 2005, vol. 57, p. 1

Doucet C., Lagage P., Pantin E., High resolution Mid-Infrared Imaging of Dust Disks Structures around Herbig Ae Stars with VISIR. In Visions for Infrared Astronomy, Instrumentation, Mesure, Métrologie , 2006, p. 25

Draine B. T., Lee H. M., Optical properties of interstellar graphite and silicate grains, ApJ, 1984, vol. 285, p. 89

Dullemond C. P., Dominik C., Natta A., Passive Irradiated Circumstellar Disks with an Inner Hole, ApJ, 2001, vol. 560, p. 957

Fujiwara H., Honda M., Kataza H., Yamashita T., Onaka T., Fukagawa M., Okamoto Y. K., Miyata T., Sako S., Fujiyoshi T., Sakon I., The Asymmetric Thermal Emission of 
the Protoplanetary Disk Surrounding HD 142527 Seen by Subaru/COMICS, ApJ, 2006, vol. 644, p. L133

Garcia-Lario P., Manchado A., Pych W., Pottasch S. R., Near infrared photometry of IRAS sources with colours like planetary nebulae. III, A\&AS, 1997, vol. 126, p. 479

Goto M., van der Plas G., van den Ancker M., Dullemond C. P., Carmona A., Henning T., Meeus G., Linz H., Stecklum B., Warm gas at 50 AU in the disk around Herbig Be star HD 100546, A\&A, 2012, vol. 539, p. A81

Grady C. A., Polomski E. F., Henning T., Stecklum B., Woodgate B. E., Telesco C. M., Piña R. K., Gull T. R., Boggess A., Bowers C. W., Bruhweiler F. C., The Disk and Environment of the Herbig Be Star HD 100546, AJ, 2001, vol. 122, p. 3396

Gregorio-Hetem J., Castilho B. V., Barbuy B., IRAS colours of Li-rich giants, A\&A, 1993, vol. 268, p. L25

Gregorio-Hetem J., Hetem A., Classification of a selected sample of weak T Tauri stars, MNRAS, 2002, vol. 336, p. 197

Gregorio-Hetem J., Lepine J. R. D., Quast G. R., Torres C. A. O., de La Reza R., A search for T Tauri stars based on the IRAS point source catalog., AJ, 1992, vol. 103, p. 549

Gutermuth R. A., Megeath S. T., Myers P. C., Allen L. E., Pipher J. L., Fazio G. G., A Spitzer Survey of Young Stellar Clusters Within One Kiloparsec of the Sun: Cluster Core Extraction and Basic Structural Analysis, ApJS, 2009, vol. 184, p. 18

Hanner M., , 1988 Technical report Grain optical properties

Hartmann L., Accretion Processes in Star Formation, 1998

Herbig G. H., The Spectra of Be- and Ae-TYPE Stars Associated with Nebulosity, ApJS, 1960, vol. 4, p. 337

Herbig G. H., Bell K. R., Third Catalog of Emission-Line Stars of the Orion Population : $3: 1988,1988$ 
Hetem A., Gregorio-Hetem J., The use of genetic algorithms to model protoplanetary discs, MNRAS, 2007, vol. 382, p. 1707

Hill T., Burton M. G., Minier V., Thompson M. A., Walsh A. J., Hunt-Cunningham M., Garay G., Millimetre continuum observations of southern massive star formation regions - I. SIMBA observations of cold cores, MNRAS, 2005, vol. 363, p. 405

Hill T., Pinte C., Minier V., Burton M. G., Cunningham M. R., Spectral energy distribution modelling of southern candidate massive protostars using the Bayesian inference method, MNRAS, 2009, vol. 392, p. 768

Holland J. H., Adaptation in Natural and Artificial Systems, 1992

Ishihara D., Onaka T., Kataza H., Salama A., Alfageme C., Cassatella A., Cox N., GarcíaLario P., et al. The AKARI/IRC mid-infrared all-sky survey, A\&A, 2010, vol. 514, p. A1

Ita Y., Matsuura M., Ishihara D., Oyabu S., Takita S., Kataza H., Yamamura I., Matsunaga N., Tanabé T., Nakada Y., Fujiwara H., Wada T., Onaka T., Matsuhara H., AKARI's infrared view on nearby stars. Using AKARI infrared camera all-sky survey, 2MASS, and Hipparcos catalogs, A\&A, 2010, vol. 514, p. A2

Ivezic Z., Elitzur M., Self-similarity and scaling behaviour of infrared emission from radiatively heated dust - I. Theory, MNRAS, 1997, vol. 287, p. 799

Jourdain de Muizon M., ISO Astrophysical Spectroscopic Database (Jourdain de Muizon, 2005), VizieR Online Data Catalog, 2005, vol. 3242, p. 0

Kamp I., Bertoldi F., CO in the circumstellar disks of Vega and beta Pictoris, A\&A, 2000, vol. 353, p. 276

Kemper F., Vriend W. J., Tielens A. G. G. M., The Absence of Crystalline Silicates in the Diffuse Interstellar Medium, ApJ, 2004, vol. 609, p. 826

Kessler-Silacci J. E., Hillenbrand L. A., Blake G. A., Meyer M. R., 8-13 $\mu \mathrm{m}$ Spectroscopy of Young Stellar Objects: Evolution of the Silicate Feature, ApJ, 2005, vol. 622, p. 404 
Knacke R. F., Fajardo-Acosta S. B., Telesco C. M., Hackwell J. A., Lynch D. K., Russell R. W., The Silicates in the Disk of beta Pictoris, ApJ, 1993, vol. 418, p. 440

Kratter K. M., Matzner C. D., Fragmentation of massive protostellar discs, MNRAS, 2006, vol. 373 , p. 1563

Krumholz M. R., Klein R. I., McKee C. F., Radiation-Hydrodynamic Simulations of Collapse and Fragmentation in Massive Protostellar Cores, ApJ, 2007, vol. 656, p. 959

Lada C. J., Star formation - From OB associations to protostars. In Star Forming Regions , vol. 115 of IAU Symposium, 1987, p. 1

Lord S D., NASA Technical Memorandum 103957, 1992

Lucy L. B., An iterative technique for the rectification of observed distributions, AJ, 1974, vol. 79 , p. 745

Malfait K., Bogaert E., Waelkens C., An ultraviolet, optical and infrared study of Herbig Ae/Be stars, A\&A, 1998, vol. 331, p. 211

Mathis J. S., Rumpl W., Nordsieck K. H., The size distribution of interstellar grains, ApJ, 1977, vol. 217, p. 425

Maury A. J., Réduction et analyse des images VISIR, 2006

Maury A. J., André P., Li Z.-Y., Probing the role of protostellar feedback in clustered star formation. Mapping outflows in the collapsing protocluster NGC 2264-C, A\&A, 2009, vol. 499 , p. 175

McKee C. F., Tan J. C., The Formation of Massive Stars from Turbulent Cores, ApJ, 2003, vol. 585 , p. 850

Meeus G., Waters L. B. F. M., Bouwman J., van den Ancker M. E., Waelkens C., Malfait K., ISO spectroscopy of circumstellar dust in 14 Herbig Ae/Be systems: Towards an understanding of dust processing, A\&A, 2001, vol. 365, p. 476

Minier V., Burton M. G., Hill T., Pestalozzi M. R., Purcell C. R., Garay G., Walsh A. J., Longmore S., Star-forming protoclusters associated with methanol masers, A\&A, 2005, vol. 429 , p. 945 
Motte F., Zavagno A., Bontemps S., Schneider N., Hennemann M., di Francesco J., André P., et al. Initial highlights of the HOBYS key program, the Herschel imaging survey of OB young stellar objects, A\&A, 2010, vol. 518, p. L77

Ossenkopf V., Henning T., Dust opacities for protostellar cores, A\&A, 1994, vol. 291, p. 943

Ossenkopf V., Henning T., Mathis J. S., Constraints on cosmic silicates, A\&A, 1992, vol. 261, p. 567

Padgett D. L., Brandner W., Stapelfeldt K. R., Strom S. E., Terebey S., Koerner D., HUBBLE SPACE TELESCOPE/NICMOS Imaging of Disks and Envelopes around Very Young Stars, AJ, 1999, vol. 117, p. 1490

Pantin E D., Observations en infrarouge de disques cirscumstellaires et d'exoplanètes, 2011

Pantin E., Waelkens C., Lagage P. O., ADONIS observations of the HD 100546 circumstellar dust disk, A\&A, 2000, vol. 361, p. L9

Pascale E., Ade P. A. R., Bock J. J., Chapin E. L., Chung J., Devlin M. J., Dicker S., Griffin M., et al. The Balloon-borne Large Aperture Submillimeter Telescope: BLAST, ApJ, 2008, vol. 681, p. 400

Pegourie B., Optical properties of alpha silicon carbide, A\&A, 1988, vol. 194, p. 335

Pereira C. B., Miranda L. F., Spectroscopic survey of post-AGB star candidates, A\&A, 2007, vol. 462, p. 231

Peretto N., Hennebelle P., André P., Probing the formation of intermediate- to high-mass stars in protoclusters. II. Comparison between millimeter interferometric observations of NGC 2264-C and SPH simulations of a collapsing clump, A\&A, 2007, vol. 464, p. 983

Perrin M. D., Vacca W. D., Graham J. R., Evidence for an Edge-on Disk Around the Young Star MWC 778 from Infrared Imaging and Polarimetry, AJ, 2009, vol. 137, p. 4468 
Pilbratt G. L., Herschel mission overview and key programmes. In Society of Photo-Optical Instrumentation Engineers (SPIE) Conference Series, vol. 7010 of Society of PhotoOptical Instrumentation Engineers (SPIE) Conference Series, 2008

Preibisch T., Ratzka T., Gehring T., Ohlendorf H., Zinnecker H., King R. R., McCaughrean M. J., Lewis J. R., Detection of a large massive circumstellar disk around a high-mass young stellar object in the Carina Nebula, A\&A, 2011, vol. 530, p. A40

Reipurth B., Yu K. C., Heathcote S., Bally J., Rodríguez L. F., Hubble Space Telescope NICMOS Images of Herbig-Haro Energy Sources: [Fe II] Jets, Binarity, and Envelope Cavities, AJ, 2000, vol. 120, p. 1449

Richardson W. H., Bayesian-Based Iterative Method of Image Restoration, Journal of the Optical Society of America (1917-1983), 1972, vol. 62, p. 55

Riera A., García-Lario P., Manchado A., Circumstellar Material around the Proto-PNe IRAS 10178-5958 and IRAS 11065-6026, Ap\&SS, 1995, vol. 224, p. 549

Robitaille T P., Star Formation across the Galaxy, PhD thesis, 2008

Robitaille T. P., Whitney B. A., Indebetouw R., Wood K., Interpreting Spectral Energy Distributions from Young Stellar Objects. II. Fitting Observed SEDs Using a Large Grid of Precomputed Models, ApJS, 2007, vol. 169, p. 328

Rodrigues C. V., Jablonski F. J., Gregorio-Hetem J., Hickel G. R., Sartori M. J., Optical Polarization and Near-Infrared Photometry of the Proto-planetary Nebula Henize 31475, ApJ, 2003, vol. 587, p. 312

Sako S., Okamoto Y. K., Kataza H., Miyata T., Takubo S., Honda M., Fujiyoshi T., Onaka T., Yamashita T., Improvements in Operating the Raytheon $320 \times 240$ Pixel Si:As Impurity Band Conduction Mid-Infrared Array, PASP, 2003, vol. 115, p. 1407

Sartori M. J., Gregorio-Hetem J., Hetem Jr. A., Classification of the Pico dos Dias Survey Herbig Ae/Be stars. In Open Issues in Local Star Formation, vol. 299 of Astrophysics and Space Science Library, 2003, p. 133 
Sartori M. J., Gregorio-Hetem J., Rodrigues C. V., Hetem Jr. A., Batalha C., Analysis of the Pico Dos Dias Survey Herbig Ae/Be Candidates, AJ, 2010, vol. 139, p. 27

Shakura N. I., Sunyaev R. A., Black holes in binary systems. Observational appearance., A\&A, 1973, vol. 24, p. 337

Siódmiak N., Meixner M., Ueta T., Sugerman B. E. K., Van de Steene G. C., Szczerba R., Hubble Space Telescope Snapshot Survey of Post-AGB Objects, ApJ, 2008, vol. 677, p. 382

Starck J.-L., Murtagh F., Astronomical image and data analysis, 2002

Szczerba R., Siódmiak N., Stasińska G., Borkowski J., An evolutionary catalogue of galactic post-AGB and related objects, A\&A, 2007, vol. 469, p. 799

Terebey S., Shu F. H., Cassen P., The collapse of the cores of slowly rotating isothermal clouds, ApJ, 1984, vol. 286, p. 529

Torres C. A. O., Publication of CNPq/Observatório Nacional (Brazil)., AJ, 1999

Torres C. A. O., Quast G., de La Reza R., Gregorio-Hetem J., Lepine J. R. D., Search for T Tauri Stars Based on the IRAS Point Source Catalog. II., AJ, 1995, vol. 109, p. 2146

Torres C. A. O., Quast G. R., Coziol R., Jablonski F., de La Reza R., Lepine J. R. D., Gregorio-Hetem J., Discovery of a Luminous Quasar in the Nearby Universe, ApJ, 1997, vol. 488, p. L19

Ueta T., Meixner M., Bobrowsky M., A Hubble Space Telescope Snapshot Survey of ProtoPlanetary Nebula Candidates: Two Types of Axisymmetric Reflection Nebulosities, ApJ, 2000, vol. 528 , p. 861

Ueta T., Murakawa K., Meixner M., Hubble Space Telescope NICMOS Imaging Polarimetry of Proto-Planetary Nebulae. II. Macromorphology of the Dust Shell Structure via Polarized Light, AJ, 2007, vol. 133, p. 1345

van Boekel R., Min M., Waters L. B. F. M., de Koter A., Dominik C., van den Ancker M. E., Bouwman J., A $10 \mu \mathrm{m}$ spectroscopic survey of Herbig Ae star disks: Grain growth and crystallization, A\&A, 2005, vol. 437, p. 189 
Verhoeff A., Dusty Disks around Young Stars, Sterrenkundig Instituuut "Anton Pannekoek", University of Amsterdam, 2009, Tese de Doutorado

Verhoeff A. P., Min M., Pantin E., Waters L. B. F. M., Tielens A. G. G. M., Honda M., Fujiwara H., Bouwman J., van Boekel R., Dougherty S. M., de Koter A., Dominik C., Mulders G. D., The complex circumstellar environment of HD 142527, A\&A, 2011, vol. 528 , p. A91

Verhoeff A. P., Waters L. B. F. M., van den Ancker M. E., Min M., Stap F. A., Pantin E., van Boekel R., Acke B., Tielens A. G. G. M., de Koter A., A mid-IR study of the circumstellar environment of Herbig Be stars, A\&A, 2012, vol. 538, p. A101

Verma R. P., Rengarajan T. N., Ghosh S. K., A two band far-infrared photometer with bolometer arrays, Bulletin of the Astronomical Society of India, 1993, vol. 21, p. 489

Vieira R G., Dissertação de Mestrado: Confirmação da natureza de candidatas Herbig Ae/Be comparação com a fase pós-ramo assintótico, 2008

Vieira R. G., Gregorio-Hetem J., Hetem A., Stasińska G., Szczerba R., Distinguishing post-AGB impostors in a sample of pre-main sequence stars, A\&A, 2011, vol. 526, p. A24

Vieira S. L. A., Corradi W. J. B., Alencar S. H. P., Mendes L. T. S., Torres C. A. O., Quast G. R., Guimarães M. M., da Silva L., Investigation of 131 Herbig Ae/Be Candidate Stars, AJ, 2003, vol. 126, p. 2971

Waters L. B. F. M., Marlborough J. M., The Structure of the Circumstellar Material in Be Stars. In Pulsation; Rotation; and Mass Loss in Early-Type Stars, vol. 162 of IAU Symposium, 1994, p. 399

Waters L. B. F. M., Waelkens C., Herbig Ae/Be Stars, ARA\&A, 1998, vol. 36, p. 233

Werner M. W., Roellig T. L., Low F. J., Rieke G. H., Rieke M., Hoffmann W. F., Young E., Houck J. R., Brandl B., Fazio G. G., Hora J. L., Gehrz R. D., The Spitzer Space Telescope Mission, ApJS, 2004, vol. 154, p. 1 
Whitney B. A., Wood K., Bjorkman J. E., Wolff M. J., Two-dimensional Radiative Transfer in Protostellar Envelopes. I. Effects of Geometry on Class I Sources, ApJ, 2003, vol. 591, p. 1049

Whittet D. C. B., Boogert A. C. A., Gerakines P. A., Schutte W., Tielens A. G. G. M., de Graauw T., Prusti T., van Dishoeck E. F., Wesselius P. R., Wright C. M., Infrared Spectroscopy of Dust in the Diffuse Interstellar Medium toward Cygnus OB2 No. 12, ApJ, 1997, vol. 490, p. 729

Wright E. L., Eisenhardt P. R. M., Mainzer A. K., Ressler M. E., Cutri R. M., Jarrett T., Kirkpatrick J. D., Padgett D., McMillan R. S., Skrutskie M., The Wide-field Infrared Survey Explorer (WISE): Mission Description and Initial On-orbit Performance, AJ, 2010, vol. 140, p. 1868

Yorke H. W., Sonnhalter C., On the Formation of Massive Stars, ApJ, 2002, vol. 569, p. 846 
Apêndice 

Apêndice A

\section{Lista de acrônimos e convenções do texto}

Este apêndice apresenta a lista de acrônimos utilizada ao longo desta tese. Decidimos manter suas versões em língua inglesa, de modo que sua associação aos trabalhos da literatura seja direta.

Também convencionamos a utilização do ponto ao invés da vírgula como separador decimal, dado que a maioria das ferramentas de manipulação de dados adotam esta notação.

Finalmente, preferimos manter os textos contidos nas figuras em inglês, visando a futura utilização destas em publicações internacionais. No mais, preferimos sempre adotar a versão portuguesa das denominações.

- 2MASS: Two Micron All Sky Survey

- AGB: ramo assintótico das gigantes

- BB: corpo negro

- BLAST: Balloon-borne Large Aperture Sub-millimeter Telescope

- COBE: Cosmic Background Explorer

- DSS: Digitized Sky Survey

- DUPLEX: DUst-Prominent Longitudinally-EXtended

- ESA: European Space Agency 
- ESO: European Southern Observatory

- FWHM: largura à meia altura

- GA: algoritmo genético

- HAeBe: Herbig Ae/Be

- HBC: Herbig-Bell Catalog

- HST: Hubble Space Telescope

- IDL: Interactive Data Language

- IR: infravermelho

- IRAS: Infrared Astronomical Satellite

- ISM: meio interestelar

- ISO: Infrared Space Observatory

- KS05: Kessler-Silacci et al. (2005)

- LRS: espectro (IRAS) de baixa resolução

- LWS: Long-Wave Spectrometer

- MIDI: mid-infrared instrument (do VLTI)

- MS: sequência principal

- MSX: Midcourse Space Experiment

- NASA: National Aeronautics and Space Administration

- NICMOS2: Near Infrared Camera and Multi Object Spectrometer (do HST)

- PAH: hidrocarboneto policíclico aromático

- PDS: Pico dos Dias Survey

- PMS: pré-sequência principal 
- PPN: proto-nebulosa planetária

- PSF: Point Spread Function

- ROB07: Robitaille et al. (2007)

- SED: Distribuição Espectral de Energia

- SIMBA: SEST IMaging Bolometer Array

- SOLE: Star-Obvious Low-level Elongated

- SWS: Short-wave Spectrometer

- TIFR: Tata Institute of Fundamental Research

- TT: T Tauri

- UV: ultravioleta

- VISIR: VLT spectrometer and imager for the mid-infrared

- VLT: Very Large Telescope

- VLTI: Very Large Telescope Interferometer

- WISE: Wide-field Infrared Survey Explorer

- WMAP: Wilkinson Microwave Anisotropy

- YSO: objeto estelar jovem 


\section{Apêndice B}

\section{Artigo publicado com resultados parciais obtidos durante o doutorado}

Neste apêndice encontra-se anexado o artigo publicado pela Astronomy E Astrophysics em Janeiro de 2011, Distinguishing post-AGB impostors in a sample of pre-main sequence stars (Vieira et al. 2011). Este trabalho apresenta o estudo detalhado da amostra de 27 objetos do Grupo 1 (Sartori et al. 2003), baseado em diagramas de cores, medidas de extinção e largura equivalente da linha de $\mathrm{H}_{\alpha}$. Grande parte deste trabalho foi desenvolvida durante o mestrado (Vieira 2008). As novas análises desenvolvidas e resultados obtidos durante o período de doutorado são descritos no Capítulo 2. 


\title{
Distinguishing post-AGB impostors in a sample of pre-main sequence stars
}

\author{
R. G. Vieira ${ }^{1}$, J. Gregorio-Hetem ${ }^{1}$, A. Hetem Jr. ${ }^{2}$, G. Stasińska ${ }^{3}$, and R. Szczerba ${ }^{4}$ \\ ${ }^{1}$ Universidade de São Paulo, IAG - Rua do Matão, 1226, 05508-900 São Paulo, SP, Brazil \\ e-mail: vieira@astro.iag.usp.br \\ 2 Universidade Federal do ABC, CECS, Rua Santa Adélia, 166, 09210-170 Santo André, SP, Brazil \\ 3 Observatoire de Meudon, LUTH, 5 Place Jules Janssen, 92190 Meudon, France \\ ${ }^{4}$ N. Copernicus Astronomical Center, Rabiańska 8, 87-100 Toruń, Poland
}

Received 16 August 2010 / Accepted 16 September 2010

\section{ABSTRACT}

\begin{abstract}
Context. A sample of 27 sources, cataloged as pre-main sequence stars by the Pico dos Dias Survey (PDS), is analyzed to investigate a possible contamination by post-AGB stars. The far-infrared excess due to dust present in the circumstellar envelope is typical of both categories: young stars and objects that have already left the main sequence and are suffering severe mass loss.

Aims. The two known post-AGB stars in our sample inspired us to seek for other very likely or possible post-AGB objects among PDS sources previously suggested to be Herbig Ae/Be stars, by revisiting the observational database of this sample.

Methods. In a comparative study with well known post-AGBs, several characteristics were evaluated: (i) parameters related to the circumstellar emission; (ii) spatial distribution to verify the background contribution from dark clouds; (iii) spectral features; and (iv) optical and infrared colors.

Results. These characteristics suggest that seven objects of the studied sample are very likely post-AGBs, five are possible post-AGBs, eight are unlikely post-AGBs, and the nature of seven objects remains unclear.
\end{abstract}

Key words. stars: AGB and post-AGB - infrared: stars - circumstellar matter - stars: pre-main sequence

\section{Introduction}

In the study of large samples of stars with unconfirmed natures, the criteria used in selecting candidates often cause sample contamination with objects that are different than those under study. This is the case of recurring confusion between pre-main sequence (pre-MS) stars and post-asymptotic giant branch (postAGB) stars. In spite of their totally different evolution, both categories of objects share common characteristics. The observed infrared (IR) excess, which originates in circumstellar dust, can be explained by re-emission of thermal radiation produced by the central source in both cases.

Instigated by the presence of two confirmed post-AGBs in a sample of possible Herbig Ae/Be (pre-MS stars of intermediate mass), we decided to analyze in detail the objects with spectral energy distributions (SEDs) that are similar to those found in evolved stars. The sample was selected from the Pico dos Dias Survey (PDS) ${ }^{1}$ by only choosing the PDS sources showing SED more luminous in the near-IR than in the optical, which is also a known characteristic of post-AGBs.

Our goal is to distinguish the very likely, the possible, and the unlikely post-AGB objects among the selected PDS sources, which could be included in "The Torun catalog of Galactic postAGB and related objects"2 compiled by Szczerba et al. (2007). We first briefly describe the circumstellar characteristics of both, young stars, and post-AGB objects in order to review similarities and differences that are relevant to the present work.

\footnotetext{
1 PDS was a search for young stars based on infrared excess.

2 http://www.ncac.torun.pl/postagb2
}

Direct imaging is the most reliable way to study the geometry of the envelopes and to establish input disk parameters for SED-fitting models. However, sensitive imagery is constrained by several observational limits, since it is difficult to achieve for large samples.

In general, the circumstellar structure of pre-MS stars is traced through indirect means like spectroscopic data, since the profile of spectral lines may be used to infer the physical conditions of line formation.

Short-term spectral and polarimetric variability in Herbig $\mathrm{Ae} / \mathrm{Be}(\mathrm{HAeBe})$ stars indicate, for instance, circumstellar nonhomogeneities (Beskrovnaya et al. 1995), rotation, winds (Catala et al. 1999), or a magnetic field (Alecian et al. 2008). The observed SEDs of HAeBes can also be used to explain their IR excesses, which are assumed to have originated in a disk and/or an envelope surrounding the central star. Different SED shapes have been used to classify the HAeBes according to the amount of IR-excess, which is one of the diagnostics of their evolutive phase in the pre-MS.

The classification schemes of HAeBes (Hillenbrand et al. 1992; Meeus et al. 2001) are based on the SED slope in the IR band, related to the amount of IR excess and the geometric distribution of dust. However, these schemes do not consider more embedded objects, which correspond to the first phases of the young stellar objects evolution. A study of several other characteristics is required to check the pre-MS nature of the candidates, since these embedded objects have quite similar SED to those classified as post-AGB.

Following Szczerba et al. (2007) to refer to the evolutionary stage after the AGB phase, we prefer to adopt the general 
term post-AGB instead of protoplanetary nebula, since we have no a priori information about whether our objects could become planetary nebulae. Post-AGB stars are luminous objects with initial mass between 0.8 and $8 M_{\odot}$. They completed their evolution in the AGB with a severe loss of mass $\left(10^{-7}\right.$ to $\left.10^{-4} M_{\odot} / \mathrm{yr}\right)$ (Winckel 2003).

When the mass-loss process has removed all the envelope around the central core, the AGB star suffers a transition from highly embedded object to a new configuration of detached envelope, causing changes in the SED shape, which acquires a double-peak profile (Steffen et al. 1998). Van der Veen et al. (1989) classified a sample of post-AGBs, suggesting four classes according to the SED. Classes I to III have increasing SED slope from optical to far-IR wavelengths, while Class IV has doublepeak SED (maxima around near-IR and mid-IR).

Ueta et al. $(2000,2007)$ have studied the characteristics of detached dust shells and different SED of post-AGBs by means of $J-K$ and $K-[25]$ colors, which respectively describe the shape of the stellar spectrum in the near-IR and the relation between the stellar (near-IR) and dust (mid-IR) peaks. According to these authors, post-AGBs can be separated into two kinds of morphologies: elliptical (SOLE) that seem to have an optically thin shell, with starlight passing through in all directions, and bipolar (DUPLEX) that have an optically thick circumstellar torus, where the starlight passes through only along the poles. Results from the HST survey of post-AGBs presented by Siódmiak et al. (2008) support this dichotomy in the morphology of the nebulosities, which was confirmed by Szczerba et al. (in prep.) for a large sample of post-AGBs. These authors again found clear differences in near-IR colors of SOLE and DUPLEX objects, which are also correlated with the SED shape: post-AGB class IV objects (double peak) are SOLE type, while classes II or III (single peak) are DUPLEX. Similar differences in SED shape are also found in pre-MS stars, indicating that the starlight is scattered in all directions (double-peak SED) or not (single-peak) (Hillenbrand et al. 1992; Malfait et al. 1998; Meeus et al. 2001).

The main goal of the present work is to analyze a selected sample of possible HAeBes that is contaminated by the presence of post-AGBs. As described in Sect. 2, the sample was extracted from the PDS catalog by selecting the sources that show a similar SED shape to post-AGBs. Seven characteristics, typical of evolved objects, were used to distinguish them from the young stars in our sample. The analysis of these characteristics is presented as follows. In Sect. 3 we discuss the association with clouds to check (i) the effects of the interstellar medium in farIR observed fluxes and (ii) the occurrence of isolated objects. Spectral features and their relation with circumstellar characteristics are discussed in Sect. 4. Optical and IR colors are evaluated and compared to known post-AGBs in Sect. 5. The main results are summarized in Sect. 6, according to the criteria used to identify the most probable evolved objects, while concluding remarks and perspectives for future work are presented in Sect. 7 .

\section{Sample selection based on IR-excess}

The Pico dos Dias Survey (PDS ${ }^{3}$ ) (Gregorio-Hetem et al. 1992; Torres et al. 1995; Torres 1999) was a search for young stars, based on the far-IR colors of T Tauri stars (TTs). Among the

\footnotetext{
${ }^{3}$ PDS was conducted at the Observatório do Pico dos Dias, which is operated by the Laboratório Nacional de Astrofísica/MCT, Brazil.
}

detection of more than 70 new TTs, PDS also provided a list of 108 HAeBe candidates published by Vieira et al. (2003).

Sartori et al. (2010) classified the HAeBe PDS candidates according to the SED slope (spectral index) and the contribution of circumstellar emission to the total flux. They verified that $84 \%$ of the studied PDS sources can be confidently considered as $\mathrm{HAeBe}$, while the nature of several candidates remains uncertain.

In this section we describe the criteria adopted to select the objects studied in the present work, by using spectral index and the fraction of circumstellar luminosity as tracers of IR-excess.

\subsection{Spectral index}

The PDS HAeBe candidates were separated by Sartori et al. (2010) into three groups, according to the SED slope between optical and mid-IR, measured by the spectral index $\beta_{1}=$ $0.75 \log \left(F_{12} / F_{\mathrm{V}}\right)-1$ (Torres 1999$)$. We selected 27 of the PDS sources of an unclear nature, mainly those with high spectral in$\operatorname{dex}\left(\beta_{1}>0.7\right)$, which correspond to the most prominent IR excesses. For objects showing single-peak SED, the spectral index is related to the level of circumstellar extinction. Even if the SED is double-peak, high values of the $\beta_{1}$ index also indicate high levels of circumstellar emission (at $12 \mu \mathrm{m}$ ), somewhat higher than the optical flux ( $V$ band), unrelated to the stellar temperature.

Among the selected sources, there are two previously known post-AGBs, Hen 3-1475 (PDS465) and IRAS19343+2926 (PDS581), according to evidence reported in the literature (Riera et al. 1995; Rodrigues et al. 2003; Bowers \& Knapp 1989), which motivated us to study their differences and similarities when compared to other objects of the sample.

The list of the selected objects is presented in Table 1, giving their identification and the parameters used in the present work such as spectral type (when available), $B-V$ excess, IR colors (using $2 M A S S, A K A R I$ and IRAS data), and equivalent width of $\mathrm{H} \alpha$ line. Intrinsic polarization obtained by Rodrigues et al. (2009) is also given, when available.

\subsection{Fraction of circumstellar luminosity}

Different evolutive classes of pre-MS stars are defined according the observed SED, which is affected by several characteristics of circumstellar envelopes, such as chemistry, size distribution of grains, and inclination of the system.

The conspicuous grain features around $3.1 \mu \mathrm{m}$ and $10 \mu \mathrm{m}$ can be checked through ISO or Spitzer data to better infer the nature of the circumstellar matter. However the lack of near-IR spectral data for our whole sample ${ }^{4}$ means that disk models cannot be constrained in the present work. For this reason, we decided to adopt a simple model (Gregorio-Hetem \& Hetem 2002) for the sole purpose of estimating the fraction of circumstellar luminosity. Regardless of envelope or disk parameters, we are interested in determining the integrated observed flux, in order to estimate the contribution of circumstellar flux as a fraction of the total flux, defined by $f_{\mathrm{Sc}}=\left(S_{\text {total }}-S_{\text {star }}\right) / S_{\text {total }}$.

Figure 1 shows the synthetic reproduction of observed SEDs in our sample, estimated from the blackbody emission of three components: a central star $\left(S_{\text {star }}\right)$, a flat passive disk $\left(S_{\mathrm{d}}\right)$, surrounded by a spherical envelope $\left(S_{\mathrm{e}}\right)$. Different temperature laws have been adopted: $T_{\mathrm{d}} / T_{\mathrm{star}} \propto\left(r_{\mathrm{d}}\right)^{-0.75}$ for the disk (Adams $\&$ Shu 1986) and $T_{\mathrm{e}} / T_{\text {star }} \propto\left(r_{\mathrm{e}}\right)^{-0.4}$ for the envelope (RowanRobinson 1986), as used by Epchtein et al. (1990) to reproduce

\footnotetext{
${ }^{4}$ Only PDS141, 465, 518, and 581 were observed by ISO or Spitzer.
} 
Table 1. List of studied stars.

\begin{tabular}{|c|c|c|c|c|c|c|c|c|c|c|c|c|}
\hline PDS & 2MASS & ST & $\begin{array}{c}E(B-V) \\
\quad \mathrm{mag}\end{array}$ & $\begin{array}{c}J-H \\
\mathrm{mag}\end{array}$ & $\begin{array}{c}H-K \\
\mathrm{mag}\end{array}$ & $\begin{array}{c}K-[25] \\
\mathrm{mag}\end{array}$ & $\begin{array}{c}J-[18] \\
\mathrm{mag}\end{array}$ & $\begin{array}{c}\text { [9] }-[18] \\
\mathrm{mag}\end{array}$ & $\begin{array}{c}{[12]-[25]} \\
\mathrm{mag}\end{array}$ & $\begin{array}{c}{[25]-[60]} \\
\mathrm{mag}\end{array}$ & $\begin{array}{c}W_{\mathrm{H} \alpha} \\
\AA\end{array}$ & $\begin{array}{l}P \\
\%\end{array}$ \\
\hline 018 & $05534254-1024006$ & B7 & 1.42 & 2.17 & 1.68 & 7.41 & 10.26 & 2.04 & 0.42 & 0.10 & 40 & - \\
\hline 027 & 07193593-1739180 & B2 & 1.41 & 1.47 & 1.32 & 8.15 & 9.78 & 2.39 & 0.84 & 0.50 & 88 & - \\
\hline 037 & $10100032-5702073$ & B2 & 1.60 & 1.86 & 1.40 & 8.28 & 10.34 & 2.55 & 1.04 & 1.00 & 105 & 2.76 \\
\hline 067 & $13524285-6332492$ & B & 1.56 & 1.59 & 1.49 & 6.23 & 8.56 & 1.51 & 0 & -0.24 & 90 & 2.73 \\
\hline 141 & $12531722-7707106$ & $?$ & - & 2.32 & 1.82 & 7.95 & 11.06 & 2.43 & 0.84 & 0.22 & 40 & \\
\hline 168 & $04305028+2300088$ & F0 & 2.14 & 1.74 & 1.19 & 6.51 & 8.53 & 1.87 & 0.19 & -0.45 & 8 & 5.84 \\
\hline 174 & 05065551-0321132 & B3 & 1.01 & 0.76 & 0.65 & 10.38 & - & - & 1.17 & 1.95 & 65 & - \\
\hline 193 & 05380931-0649166 & B9 & 1.34 & 0.97 & 0.99 & 7.20 & 8.18 & 1.86 & 0.16 & 0.52 & 12 & 2.25 \\
\hline 198 & 05385862-0716457 & F0 & 1.13 & 1.03 & 1.01 & 6.95 & 7.60 & 2.79 & 1.70 & 1.07 & 9 & - \\
\hline 204 & $05501389+2352177$ & B1 & 1.10 & 1.24 & 1.22 & 9.68 & 10.73 & 3.31 & 2.00 & 0.96 & 245 & - \\
\hline 207 & $06071539+2957550$ & $\mathrm{~B} ?$ & 1.20 & 1.18 & 1.21 & 8.20 & - & - & 0.62 & 1.77 & 5 & - \\
\hline 216 & $06235631+1430280$ & B2 & 1.19 & 1.32 & 1.28 & 8.14 & 9.59 & 1.65 & 0.33 & 2.03 & 200 & - \\
\hline 257 & 07414105-2000134 & A & 0.89 & 1.20 & 1.21 & 7.81 & - & - & 0.35 & 1.77 & 15 & - \\
\hline 290 & $09261107-5242269$ & A & 0.66 & 0.35 & 0.28 & 10.9 & - & - & 0.26 & 2.33 & -7 & 1.74 \\
\hline 353 & $12222318-6317167$ & B5 & 0.99 & 1.31 & 1.17 & 8.28 & 9.68 & 1.97 & 0.45 & 2.00 & 200 & 2.17 \\
\hline 371 & $13473141-3639495$ & O9? & 1.40 & 1.74 & 1.31 & 7.17 & 9.18 & 2.36 & 0.58 & -0.1 & 40 & \\
\hline 394 & $15351712-6159041$ & F0 & 0.44 & 0.51 & 0.52 & 11.45 & 11.17 & 3.34 & 1.51 & -0.7 & -2 & 2.25 \\
\hline 406 & $16050392-3945034$ & A5 & 0.40 & 1.08 & 1.05 & 8.33 & 9.19 & 1.95 & 0.79 & 1.94 & 13 & 3.20 \\
\hline 431 & $16545918-4321496$ & A0 & 0.52 & 0.17 & 0.16 & 13.88 & - & - & 2.00 & 2.29 & 8 & 0.23 \\
\hline 465 & $17451419-1756469$ & B & 1.23 & 1.36 & 1.48 & 8.37 & 9.73 & - & 1.51 & 0.88 & 110 & 7.88 \\
\hline 477 & $18003031-1647259$ & B1 & 1.37 & 1.43 & 1.19 & 7.42 & 9.08 & 2.22 & 0.41 & -0.01 & 120 & 1.40 \\
\hline 518 & $18273952-0349520$ & $\mathrm{OB}$ & 2.30 & 1.74 & 1.35 & 6.85 & - & - & 0.37 & 1.53 & 600 & 3.80 \\
\hline 520 & $18300616+0042336$ & F3 & 1.26 & 1.23 & 1.09 & 6.88 & 8.13 & 2.21 & 0.70 & 0.47 & 33 & 3.67 \\
\hline 530 & $18413436+0808207$ & A5 & 0.47 & 1.59 & 1.66 & 7.93 & 9.92 & 2.12 & 0.52 & -0.07 & 28 & 11.06 \\
\hline 543 & $18480066+0254170$ & B0 & 1.79 & 0.48 & 0.40 & 8.91 & 8.93 & 3.82 & 1.92 & 0.41 & 0.8 & 1.14 \\
\hline 551 & $18552297+0404353$ & B0 & 1.92 & 1.53 & 1.26 & 8.16 & 9.49 & 2.15 & 0.57 & 0.57 & 50 & 11 \\
\hline 581 & $19361890+2932500$ & B1 & 0.89 & 1.98 & 1.72 & 8.58 & 10.82 & - & 1.33 & 0.74 & 200 & 12.22 \\
\hline
\end{tabular}

Notes. Column description: (1) PDS name; (2) 2MASS identification; (3) spectral type; (4) $B-V$ excess; (5-11) infrared colors; (12) $\mathrm{H}_{\alpha}$ equivalent width (negative values represent absorption lines); (13) intrinsic polarization.

the IR data of carbon stars. The observational data are from $P D S$ (optical photometry), 2MASS, MSX, IRAS, AKARI, and ISO catalogs. Three curves calculated by the adopted model, without fitting purpose, illustrate the expected variation in the fraction of circumstellar luminosity. In the case of PDS465, for example, $f_{\mathrm{Sc}}$ varies from 0.90 to 0.94 , which is the typical dispersion found in our sample, leading us to adopt an error of 0.02 in the estimation of $f_{\mathrm{Sc}}$.

All objects in our sample have $\beta_{1}>0.7$ and $f_{\mathrm{Sc}}>0.7$ that correspond to large amounts of circumstellar emission, suggesting two possible scenarios as embedded pre-MS stars or possible post-AGBs.

We are aware that it is important to check that the data points around $100 \mu \mathrm{m}$ reflect the cloud and are not in the immediate vicinity of the star, due to the large IRAS beam size in these bands, mainly for objects lacking of ISO spectra or AKARI data, for example. In the next section we analyze the background contribution from clouds, aiming to avoid a possible contamination of interstellar matter in the far-IR data.

\section{Association with dark clouds}

The star formation process is typically associated with clouds, where the gravitational collapse of pre-stellar cores gives rise to the embedded protostars. Thus, the association with these clouds may indicate a young-star nature. Unfortunately, only rough distance measurements are available for our sample
(Vieira et al. 2003), which makes the distinction between a true association from a projection effect uncertain. Due to this restriction, we decided to evaluate the probability of association with clouds, where the objects apparently closer to dark clouds probably have a young nature (age $<2 \mathrm{Myr}$ ), while stars located far from clouds are expected to be evolved objects.

However, this cannot be used as a deterministic characteristic, since several examples of isolated pre-MS stars are known, such as AB Aur, HD 163296, and HD100546 (e.g., van den Ancker 1999). Our main goals in investigating the spatial relation to dark clouds are twofold: (i) to verify the occurrence of isolated objects in our sample and (ii) to estimate a possible far-IR background contamination.

\subsection{Distance to the edge of nearest clouds}

Aiming to infer the association with dark clouds, we made use of the catalog information compiled by Lynds (1962) and by Feitzinger \& Stüwe (1984). These complementary works allowed us to find the dark clouds closer to the objects of our sample. An opacity class number, which ranges from 1 to 6 , is assigned to each dark cloud of these catalogs. We adopted the criterion of choosing the closest dark cloud at least with opacity class 3 . This choice restricts the selected clouds to those with a relevant extinction level, although it must be kept in mind that these opacity classes were defined by visual inspection of photographic plates. Given the selection of the closest 

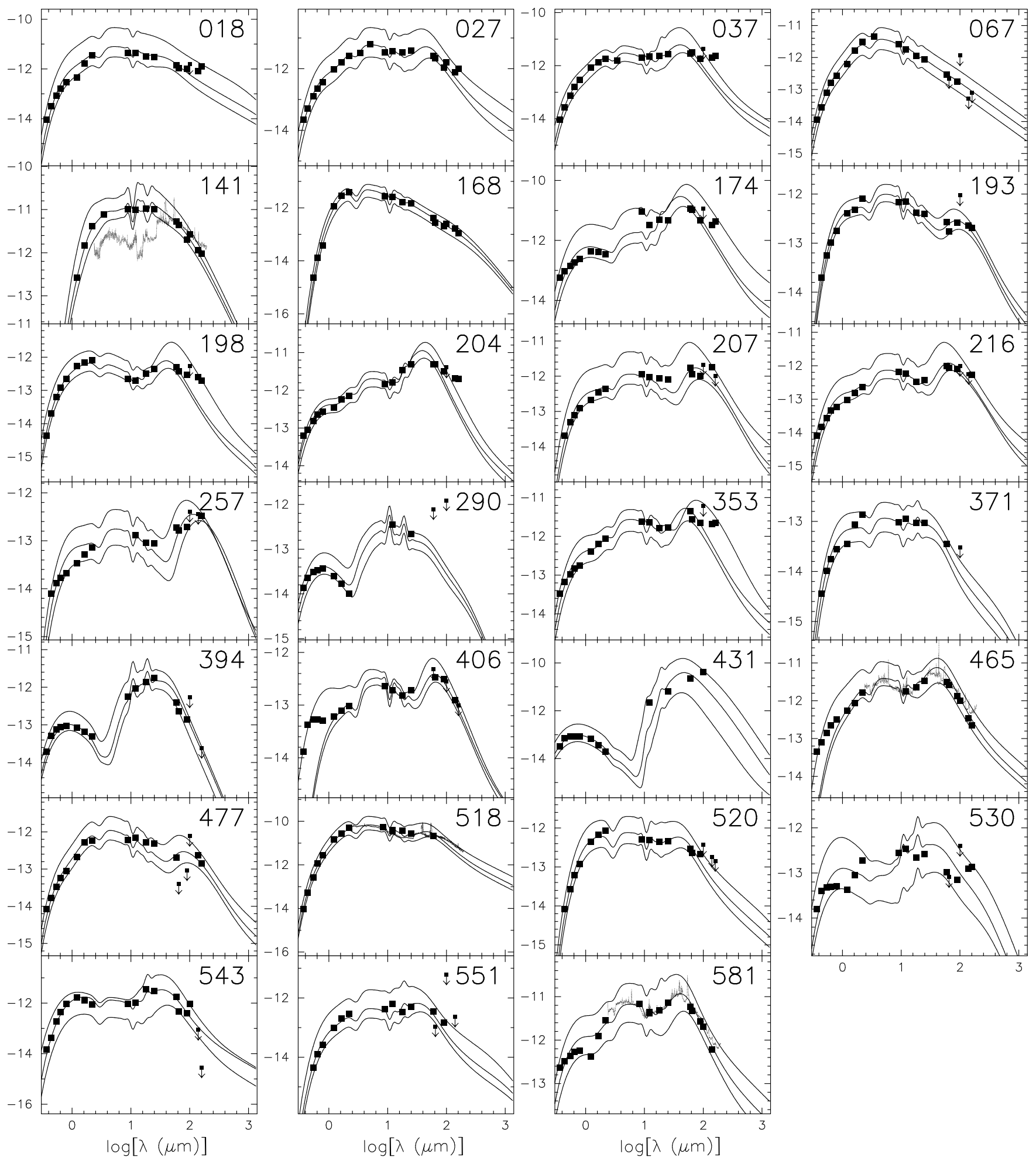

Fig. 1. Observed SED of our sample showing $\log \left(\lambda \mathrm{F}_{\lambda}\right.$ in $\left[\mathrm{W} \mathrm{m}^{-2}\right]$ vs. $\log (\lambda)$ in $[\mu \mathrm{m}]$. Filled squares represent optical photometry, $2 M A S S, A K A R I$, $M S X$ and IRAS data, while dots are used to plot ISO spectra. Solid curves indicate the variation in the calculated SED.

clouds, we estimated their area and distance to each star of our sample. These data allowed us to roughly estimate the distance of our objects to the border of the selected clouds, defined by $d_{\text {edge }}=D-0.5 \times a^{1 / 2}$, where $D$ stands for the projected distance from the star to the cloud's center. In this way, a negative value for $d_{\text {edge }}$ means that a star is found "inside" the angular region enclosed by the cloud of area $a$. A square area was adopted in this first-order calculation, which does not consider the actual shape of the cloud, which usually presents a filamentary geometry. Nevertheless, $d_{\text {edge }}$ quantifies a possible association with the dark cloud.

\subsection{Reddening}

Since the information obtained from catalogs of dark clouds is related to arbitrary opacities, we adopted two other methods of 
Table 2. Parameters used in the sample analysis

\begin{tabular}{|c|c|c|c|c|c|c|c|c|}
\hline PDS & $f_{\mathrm{Sc}}$ & cloud & $d_{\substack{\text { edge } \\
o}}$ & $\begin{array}{c}A_{\mathrm{V}} \\
\mathrm{mag}\end{array}$ & $f_{A v}$ & $\begin{array}{c}\langle F\rangle \\
\text { Jy }\end{array}$ & $f_{F 100}$ & Notes \\
\hline 018 & 0.90 & L1647 & 1.79 & $1.3 \pm 0.3$ & 0.7 & $20 \pm 2$ & 0.7 & $\mathrm{a}, \mathrm{b}$ \\
\hline 027 & 0.96 & L1659 & 5.60 & $1.2 \pm 0.2$ & 0.7 & $20 \pm 3$ & 0.7 & $a, b, c, f, g$ \\
\hline 037 & 0.93 & FS134 & -0.15 & $2.8 \pm 0.4$ & 0.4 & $156 \pm 41$ & $<0.5$ & e, g \\
\hline 067 & 0.97 & FS251 & 1.39 & $1.4 \pm 0.3$ & 0.7 & $300 \pm 101$ & $<0.1$ & $\mathrm{a}, \mathrm{f}$ \\
\hline 141 & 0.97 & FS221 & 0.09 & $3.8 \pm 0.8$ & 0 & $14 \pm 2$ & 0.9 & \\
\hline 168 & 0.75 & L1536 & -0.10 & $0.9 \pm 0.6$ & 0 & $14 \pm 1$ & 0.4 & \\
\hline 174 & 0.97 & L1616 & 0.07 & $1.2 \pm 0.2$ & 0.6 & $6 \pm 4$ & 1.0 & $\mathrm{~b}, \mathrm{c}, \mathrm{d}, \mathrm{e}, \mathrm{g}$ \\
\hline 193 & 0.70 & L1641 & -1.02 & $2.6 \pm 0.6$ & 0.4 & $37 \pm 16$ & 0.7 & \\
\hline 198 & 0.85 & L1641 & -0.76 & $2.6 \pm 0.6$ & 0.3 & $37 \pm 16$ & $<0.6$ & \\
\hline 204 & 0.95 & L1570 & 5.91 & $0.9 \pm 0.4$ & 0.7 & $17 \pm 1$ & 0.9 & $\mathrm{a}, \mathrm{b}, \mathrm{c}, \mathrm{e}, \mathrm{g}$ \\
\hline 207 & 0.89 & L1557 & -0.28 & $0.3 \pm 0.5$ & 0.9 & $16 \pm 2$ & 0.8 & $\mathrm{~b}, \mathrm{~g}$ \\
\hline 216 & 0.92 & L1600 & 1.96 & $0.8 \pm 0.2$ & 0.8 & $18 \pm 2$ & $<0.7$ & $\mathrm{a}, \mathrm{b}, \mathrm{c}, \mathrm{d}, \mathrm{g}$ \\
\hline 257 & 0.89 & L1665 & 3.70 & $0.6 \pm 0.1$ & 0.8 & $21 \pm 2$ & 0.4 & a \\
\hline 290 & 0.94 & FS114 & 1.27 & $1.6 \pm 0.2$ & 0.2 & $37 \pm 9$ & 0.5 & $\mathrm{a}, \mathrm{f}, \mathrm{g}$ \\
\hline 353 & 0.89 & FS220 & 3.61 & $1.3 \pm 0.2$ & 0.6 & $80 \pm 26$ & $<0.7$ & $\mathrm{a}, \mathrm{b}, \mathrm{c}, \mathrm{d}, \mathrm{g}$ \\
\hline 371 & 0.79 & FS271 & 18.18 & $0.1 \pm 0.1$ & 1.0 & $5 \pm 0$ & $<0.2$ & $\mathrm{a}$ \\
\hline 394 & 0.86 & FS288 & 2.46 & $0.7 \pm 0.2$ & 0.5 & $49 \pm 5$ & $<0.3$ & $\mathrm{a}, \mathrm{e}, \mathrm{g}$ \\
\hline 406 & 0.82 & FS348 & -0.20 & $1.2 \pm 0.5$ & 0 & $35 \pm 5$ & 0.2 & d, g \\
\hline 431 & 0.94 & FS372 & 1.42 & $1.6 \pm 0.4$ & 0 & $439 \pm 155$ & $<0.8$ & $\mathrm{a}, \mathrm{e}, \mathrm{f}, \mathrm{g}$ \\
\hline 465 & 0.92 & $\mathrm{~L} 273$ & 0.58 & $0.6 \pm 0.2$ & 0.8 & $30 \pm 3$ & 0.7 & $\mathrm{a}, \mathrm{b}, \mathrm{c}, \mathrm{d}, \mathrm{e}, \mathrm{f}, \mathrm{g}$ \\
\hline 477 & 0.87 & L318 & 0.10 & $2.6 \pm 0.4$ & 0.4 & $120 \pm 14$ & 0.2 & d \\
\hline 518 & 0.91 & L511 & 0.09 & $5.4 \pm 1.0$ & 0.2 & $105 \pm 11$ & 0.9 & d \\
\hline 520 & 0.84 & L571 & 0.49 & $5.5 \pm 0.8$ & 0 & $79 \pm 26$ & $<0.1$ & \\
\hline 530 & 0.82 & L637 & 0 & $1.0 \pm 0.3$ & 0.3 & $38 \pm 5$ & 0.3 & $\mathrm{f}$ \\
\hline 543 & 0.94 & L621 & -3.01 & $4.6 \pm 0.6$ & 0.2 & $164 \pm 47$ & 0.2 & $e, f, g$ \\
\hline 551 & 0.96 & L628 & 0.30 & $6.3 \pm 0.7$ & 0 & $196 \pm 43$ & $<0.5$ & $\mathrm{f}, \mathrm{g}$ \\
\hline 581 & 0.94 & L813 & 1.28 & $0 \pm 0.1$ & 1.0 & $21 \pm 3$ & 0.8 & $\mathrm{a}, \mathrm{b}, \mathrm{c}, \mathrm{d}, \mathrm{e}, \mathrm{f}, \mathrm{g}$ \\
\hline
\end{tabular}

Notes. Column description: (1) PDS name; (2) fraction of circumstellar luminosity; $(3,4)$ nearest cloud and respective distance to the edge; (5) visual extinction from Dobashi et al. (2005); (6) "net" visual extinction; $(7,8)$ background mean flux and fraction of source emission at $100 \mu \mathrm{m}$. (a) $d_{\text {edge }}>0.5^{\circ}$; (b) $f_{\mathrm{Av}}>0.5$ and $f_{F 100}>0.5$; (c) high values of $W_{\mathrm{H} \alpha}, f_{\mathrm{Av}}$ and $f_{F 100}$; (d) U-B excess; (e) [12] $-[25]>1$ and $F_{12}>2 \mathrm{Jy}$; near-IR colors typical of post-AGB: (f) $J-H$ and $H-K$, (g) $J-K$ and $K-$ [25].

evaluating dark cloud effects on the objects of our sample. First we used an extinction map, which quantifies the cloud obscuration. In the second method, we estimated the color excess to obtain the total extinction due to both interstellar (clouds) and circumstellar (disk/envelope) extinction. These two methods are described below.

\subsubsection{Extinction map}

We adopted the Galactic map of visual extinction derived by Dobashi et al. (2005) using the classical star count method on the optical database of the Digitized Sky Survey I (DSS). The map ${ }^{5}$ provides the value of the extinction $A_{\mathrm{V}}$ for the region defined by $|b| \leq 40^{\circ}$. For each object, we took the $A_{\mathrm{V}}$ value and its mean dispersion in a neighboring region of 1 square degree, aiming to quantify the fluctuation of this extinction level in the interstellar medium. This quantity reveals the presence of a possible progenitor dark cloud behind the selected object.

These extinctions and respective dispersions are given in Table 2. For illustration, some specific regions of the Dobashi et al. map are shown in Fig. 2. It must be stressed that a possible association of our objects with clouds may come from projection effects, where the dark cloud and the star are just on the same line of sight but not physically associated. However, since we do not know the distances of our objects, we can only speak in terms of probability of association. Objects having low values

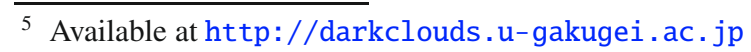

of $A_{\mathrm{V}}$ probably are isolated, but other methods are required to confirm this information.

\subsubsection{Color excess}

The visual extinction may also be estimated by the color excess that is caused by the absorption and scattering of the emitted light, occurring not only in the ISM but also (and sometimes predominantly) in the circumstellar environment of our sample. To estimate this total extinction, we evaluated $E(V-I)$ instead of $E(B-V)$, since the color excess on $V-I$ is less affected by the ultraviolet excess (Strom et al. 1975). The intrinsic colors were selected from Bessel et al. (1998) for different sets of effective temperature and surface gravity.

Based on the spectral type given in the PDS catalog, an estimative of effective temperatures was obtained by adopting the empirical spectral calibration provided by de Jager \& Nieuwennhuijzen (1987). Considering that the luminosity classes are uncertain for most objects in our sample, the color excess was estimated by assuming two possible classes: main sequence and supergiant, which are adopted to represent HAeBes and post-AGBs, respectively. Then, for each pair of luminosity class and $T_{\text {eff }}$, we established a $\log g$ value extracted from Straizys \& Kuriliene (1981), enabling the most convenient choice of intrinsic colors. The $E(V-I)$ values were converted to $E(B-V)$ by the relation derived by Schultz \& Wiemer (1975): $E(B-V)=E(V-I) /(1.60 \pm 0.03)$. A normal reddening $R_{\mathrm{V}}=3.14 \pm 0.10$, which is a mean value of the interstellar 

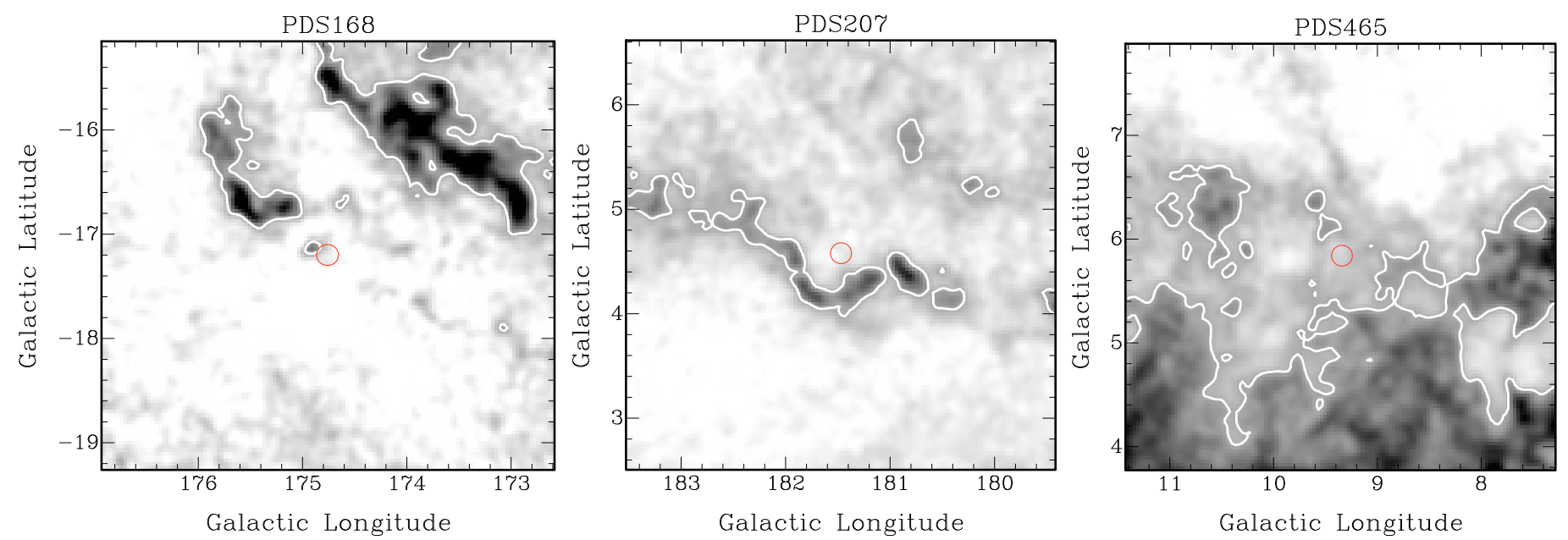

Fig. 2. Position of some of the objects (displayed by the central circle) in the Galactic extinction map produced by Dobashi et al. (2005). The halftone gray color varies from 0 to $3 \mathrm{mag}$, while the contours show $A_{\mathrm{V}}=1.0$ mag level.

extinction for several directions of the Galaxy studied by Schultz \& Wiemer (1975), was adopted to estimate the total extinction that includes both ISM and circumstellar effects, given by $A_{\mathrm{Vtot}}=3.14 \times E(B-V)$. These are minimum values, since anomalous extinction $\left(R_{\mathrm{V}}>4\right.$, for example) may be found in dense interstellar clouds (e.g. Savage \& Mathis 1979). We estimate that $A_{\mathrm{Vtot}}$ can deviate by about $50 \%$.

\subsection{Background contribution}

Far-infrared emission may also reveal the contamination by interstellar material on the observed SED. To evaluate the effects of background contamination, we used IRAS images at $100 \mu \mathrm{m}$, which is dominated by the ISM cirrus emission. For each IRAS image that contains one or more of the selected objects, we chose a region of 1 square degree in the neighborhood of the point sources, in order to estimate the background mean density flux and its standard deviation for each object. These background regions were selected avoiding the contamination of point sources in a radius of $1^{\circ}$. Table 2 gives the mean fluxes at $100 \mu \mathrm{m}(\langle F\rangle)$ obtained in the background regions.

To quantify the excess of $100 \mu \mathrm{m}$ flux we compared the flux density of the point source $\left(F_{100}\right)$ with the mean background flux explained above, defining the fraction $f_{F 100}=F_{100} /\left(F_{100}+\langle F\rangle\right)$ as an indication of flux excess. If $f_{F 100} \sim 0$ the ISM dominates the local emission, while $f_{F 100} \sim 1$ corresponds to an infrared excess due mainly to the circumstellar contribution.

We also evaluated the fraction of "net" visual extinction, which roughly corresponds to the optical depth of the circumstellar shell, by subtracting the interstellar obscuration $\left(A_{\mathrm{V}}\right)$ from the total extinction $\left(A_{\mathrm{Vtot}}\right)$. In this case, we defined $f_{A \mathrm{v}}=$ $\left(A_{\mathrm{Vtot}}-A_{\mathrm{V}}\right) / A_{\mathrm{Vtot}}$, where the interstellar extinction is obtained from the map of Dobashi et al. (2005) and the total extinction is calculated from the color excess estimated from magnitudes and spectral types given in the PDS catalog (see Sect. 3.2.2). In this case, the effects of the adopted class of luminosity in the color excess estimation are negligible.

Table 2 shows the estimated values of $f_{A_{\mathrm{v}}}$ and $f_{F 100}$. Upper limits of $f_{F 100}$ were estimated for sources having bad quality of $100 \mu \mathrm{m}$ data. Excepting PDS518, all the sources with well determined $f_{F 100}$ tend to show circumstellar extinction increasing with high levels of source emission. The two known post-AGB objects of our sample have $f_{F 100}>0.5$ and $f_{A_{v}}>0.5$, meaning that circumstellar material has respectively prominent infrared flux and optical depth, significantly above the levels found in the corresponding background. We consider these high levels of $f_{A_{v}}$ and $f_{F 100}$ characteristics of post-AGBs.

\section{Spectral features}

Optical spectra, obtained by the PDS team ${ }^{6}$, are shown in Fig. 3 for the spectral regions containing [OI] $(6302,6365 \AA)$ and [SII] (6718, $6732 \AA$ ) nebular lines, among others. The spectra of some sources are not displayed for different reasons: they have no emission lines except for $\mathrm{H} \alpha$ (PDS174, 198, 290, 394, 520, and 543 ), they are too noisy (PDS168, 207, and 431), the 6300$6500 \AA$ range was not covered (PDS18, 27, 37, 67, and 141), or the spectrum is not available (PDS257).

Almost half of our sample shows the [OI] $6302 \AA$ emission line, but only PDS371 shows features similar to those found in evolved objects like planetary nebulae (Pereira \& Miranda 2005). Considering the absence or unclear detection of these features for the whole sample, they were not used to diagnose the nature of our objects.

The equivalent width of the $\mathrm{H}_{\alpha}$ line $\left(W_{\mathrm{H} \alpha}\right)$ of the objects in our sample was measured whenever possible and reported in Table 1. In the case of spectra showing low signal-to-noise, we used the $\mathrm{W}_{\mathrm{H} \alpha}$ published in the PDS catalog.

Most of the spectra show strong $\mathrm{H}_{\alpha}$ emission lines, but two of them appear in absorption (PDS290 and 394). Line variability is a possible explanation to the weak $\mathrm{H}_{\alpha}$, which is usually seen in pre-MS stars.

A comparison of the fraction of circumstellar luminosity $\left(f_{\mathrm{Sc}}\right)$ with the strength of $\mathrm{H}_{\alpha}$ line is shown in Fig. 4. The distribution of objects with $W_{\mathrm{H} \alpha}<50 \AA$ spreads along the whole range of $f_{\mathrm{Sc}}$. On the other hand, objects showing strong emission line are mainly concentrated in the region of $f_{\mathrm{Sc}}>0.87$. As illustrated in Fig. 4, a similar distribution is found in the diagram of $W_{\mathrm{H} \alpha}$ in function of $f_{A v}$, which indicates the optical depth of the circumstellar shell, discussed in Sect. 3.3.

6 The original files were made available by Vieira, on the website http: //www. fisica.ufmg.br/ svieira/TRANSF/ 
R. G. Vieira et al.: Post-AGB impostors in a sample of pre-main sequence stars

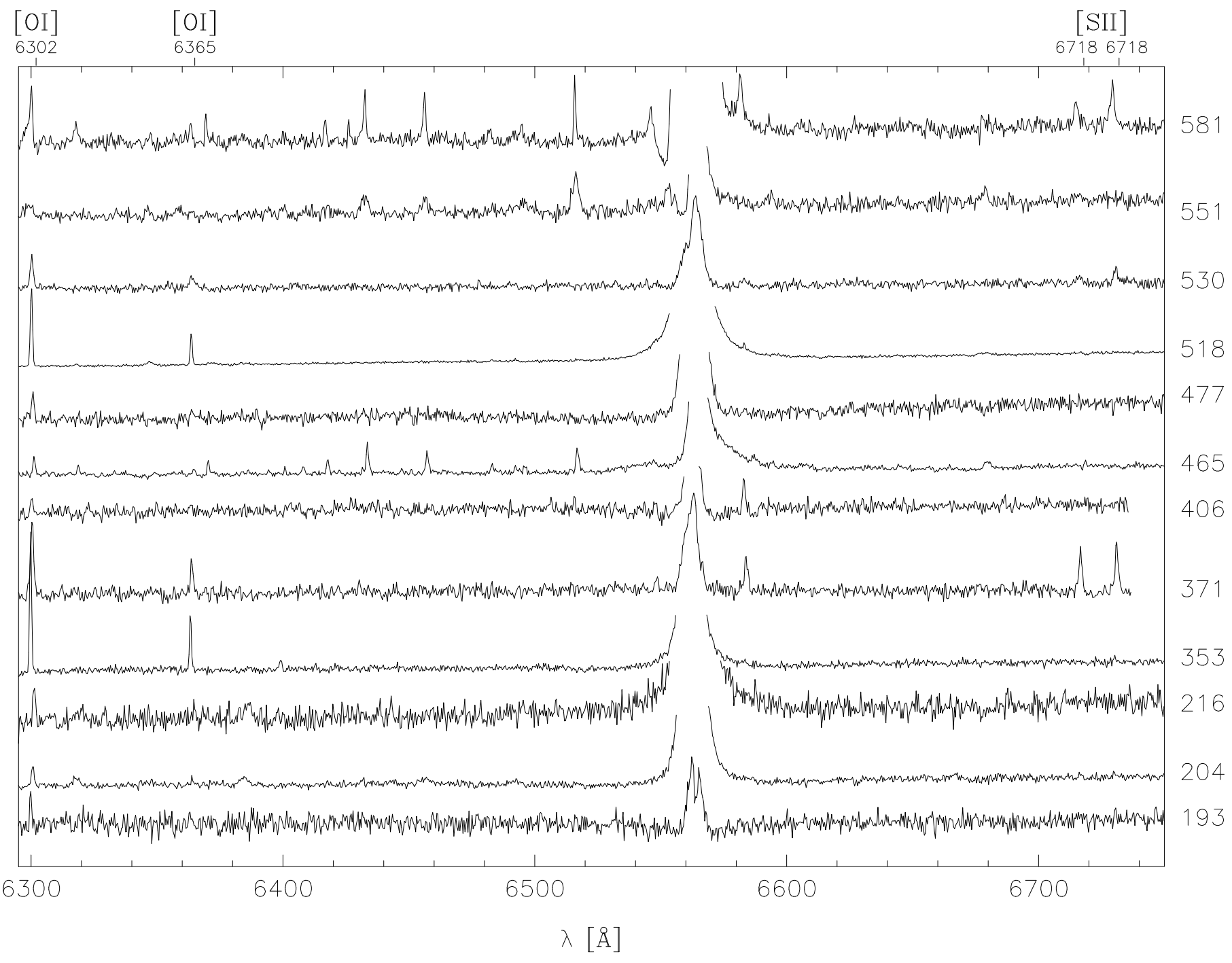

Fig. 3. Optical spectra of part of the sample, identified by the PDS number in the right side. The H $\alpha$ feature is not shown in order to enhance details of other emission lines. Intensity scale is arbitrary.

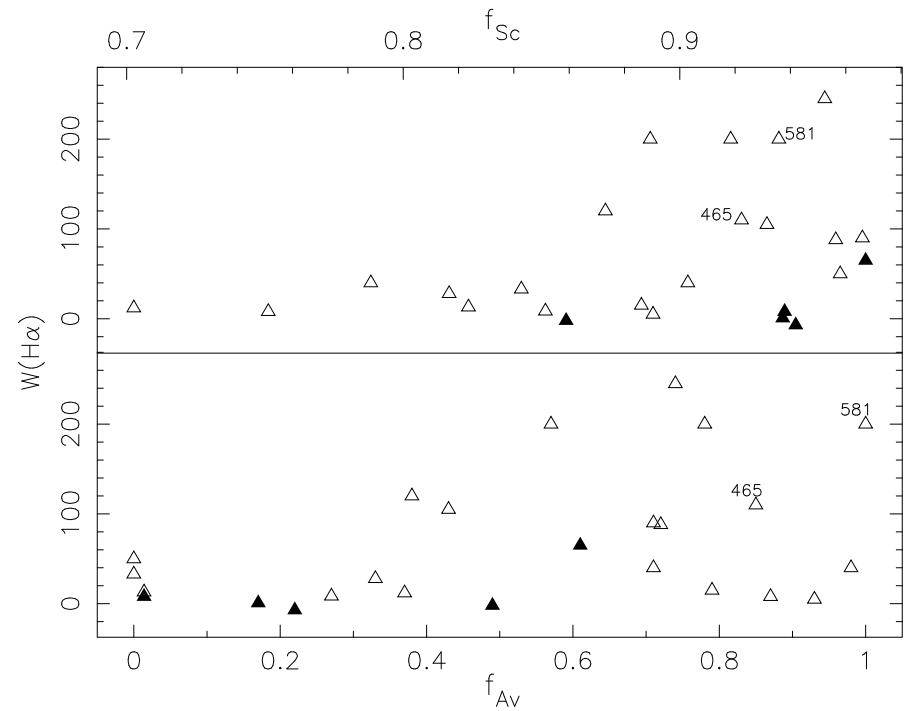

Fig. 4. Equivalent width of $\mathrm{H}_{\alpha}$ line compared to $f_{\mathrm{Sc}}($ top $)$ and $f_{A v}$ (bottom) for our stars, excepting PDS518, which has $W\left(\mathrm{H}_{\alpha}\right)=600 \AA$ A. Filled triangles are used to show the PDS sources with double peaks in the SED, while open triangles represent single peaks.

\section{Analysis of color-color diagrams}

To understand the nature of our objects it is necessary to carry out a multi-wavelength analysis. For this purpose, we used two-color diagrams in several spectral ranges. The photometric optical data were obtained from the PDS catalog. The measurements were taken in the Johnson-Cousins photometric system $U B V(R I)_{\mathrm{c}}$, with the high-speed, multicolor photometer (FOTRAP) described by Jablonski et al. (1994).

The catalogs $2 M A S S$, IRAS, MSX, and AKARI (when available) provided the data for the infrared color analysis of our objects. Optical and near-IR photometric data were corrected for extinction based on the $A_{V \text { tot }}$ calculated in Sect. 3.2.2, adopting the $A_{\lambda} / A_{\mathrm{V}}$ relations from Cardelli et al. (1989).

\subsection{Optical colors}

The spectral region represented by the $B-V$ vs. $U-B$ diagram is dominated by the photospheric radiation, which makes it very interesting when studying the nature of the central source. The optical color diagram is shown in Fig. 5 displaying those of our objects that have available $U B V$ magnitudes. For each object, two different pairs of colors are plotted according to the 


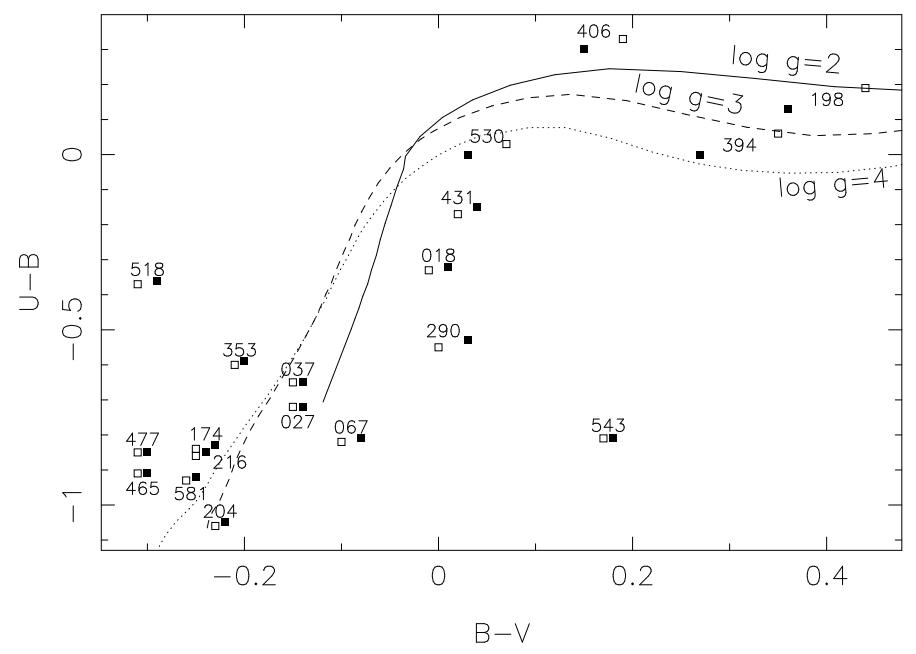

Fig. 5. Optical colors of the studied sample, after dereddening using different luminosity classes: main sequence (filled squares) and supergiants (open squares). The intrinsic colors from Bessel et al. (1998) are displayed for different surface gravities.

extinction correction explained in Sect. 3.2.2 (by adopting two luminosity classes). Superimposed on this distribution are the theoretical curves for $\log g=2,3$, and 4 calculated by Bessel et al. (1998).

It is expected that HAeBes would have $\log g \simeq 4$, similar to main sequence stars, whereas the post-AGB would have $\log g<2$, typical of supergiants. However, as shown in Fig. 5 the theoretical curves are clearly separated only for positive $B-V$ values, due to the Balmer discontinuity, which is sensitive to surface gravity in this spectral range, because it is more significant for supergiants. In this case, only PDS406 seems to satisfy the gravity criterion. On the other hand, for $B-V \lesssim 0$, where part of our objects are located, the intrinsic colors from models for $\log g=3$ and 4 are similar. In this case, the values above the theoretical lines were considered $U-B$ excess typical of evolved objects. This excess is observed for PDS465 and 581, the postAGBs of our sample, as well as PDS174, 216, 353, 477, and 518.

\subsection{Infrared colors}

The IR colors probe the thermal emission from grains that comes from dust at different temperatures: (i) cold grains found in circumstellar shells at temperatures lower than $300 \mathrm{~K}$ or (ii) warm dust $(T \sim 1000 \mathrm{~K})$ produced by a recent episode of mass loss in the case of post-AGBs, or from a protostellar disk in pre-MS stars.

\subsubsection{Far-IR}

Diagrams of IR colors have been used to identify post-AGB candidates according to their expected locus, which is related to their evolution. Van der Veen \& Habing (1988) suggested an evolutive sequence in a diagram of IRAS colors for post-AGB stars, defined by their mass-loss rate, in a scenario of shortperiod Miras evolving into long-period $\mathrm{OH} / \mathrm{IR}$ stars. However, this hypothesis is based on observational results that are not firmly supported, since the distances to OH/IR stars are uncertain (Whitelock et al. 1991). Other authors (e.g. Feast \& Whitelock 1987) favor other scenarios in which the Mira period is a function of its initial mass, where few changes in period

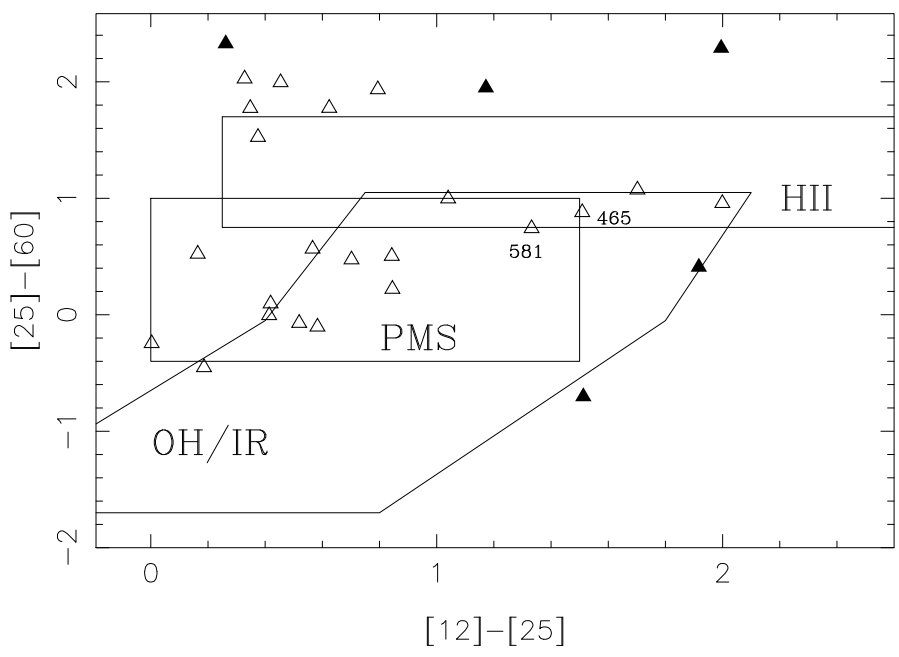

Fig. 6. Far-IR color-color diagram showing the regions for different categories of objects (GL97). Filled triangles are used to show the PDS sources with a double peak in the SED, while open triangles represent single peak.

or luminosity occur during the Mira evolution. This second scenario is supported by the results obtained by Whitelock et al. (1991), on the evolution of Miras and the origin of their periodluminosity relationship.

García-Lario et al. (1997), hereafter GL97, analyzed a sample of post-AGBs comparing their position in the [12] - [25] vs. [25] - [60] diagram with several categories of objects, such as OH/IR variables (Sivagnaman 1989), pre-MS stars (Harris et al. 1988), and compact HII regions (Antonopoulos \& Pottasch 1987).

Figure 6 shows the distribution of our sample in the IRAS color-color diagram, with respect to the regions occupied by different classes of objects, as proposed by GL97. About $70 \%$ of our objects are located in the left part $([12]-[25]<1)$ of this diagram, coinciding with the pre-MS box or above the HII region box $([25]-[60] \geq 1.5)$. Our remaining objects, among them PDS465 and 581, are found approximately in the right side of the diagram $([12]-[25] \geq 1)$. The $12 \mu \mathrm{m}$ flux density distribution for IRAS sources located in this part of the diagram was studied by van der Veen et al. (1989, see region IV in their Fig. 1). Based on the spatial distribution compared to the number of sources as a function of the $12 \mu \mathrm{m}$ flux density, they proposed a separation of objects in this region of the IRAS color diagram. According to van der Veen et al. (1989), young stars, which are associated to clouds, are those sources with $F_{12}<2$ Jy, while post-AGBs have $F_{12}>2 \mathrm{Jy}$.

\subsubsection{Mid-IR}

The fluxes at 9, 18, 65, 90, 140, and $160 \mu \mathrm{m}$ from the AKARI All Sky Survey (Ishihara et al. 2010) have been obtained for $78 \%$ of our sample. These data were used in the SED fitting discussed in Sect. 2.2. Following Ita et al. (2010) we verified the position of our sample in the $J-[18]$ vs. [9] - [18] diagram, expressed in Vega magnitudes. In Fig. 7, the AKARI colors of our sources are compared with the distribution of the very likely post-AGB stars from the Torun catalog (Szczerba et al. 2007). The position of young objects is also displayed in this diagram by plotting the YSOs from the Spitzer survey of young stellar clusters (Gutermuth et al. 2009) and the HAeBe stars from the Herbig $\&$ Bell Catalog $(H B C, 1988)$. In spite of the better quality of 


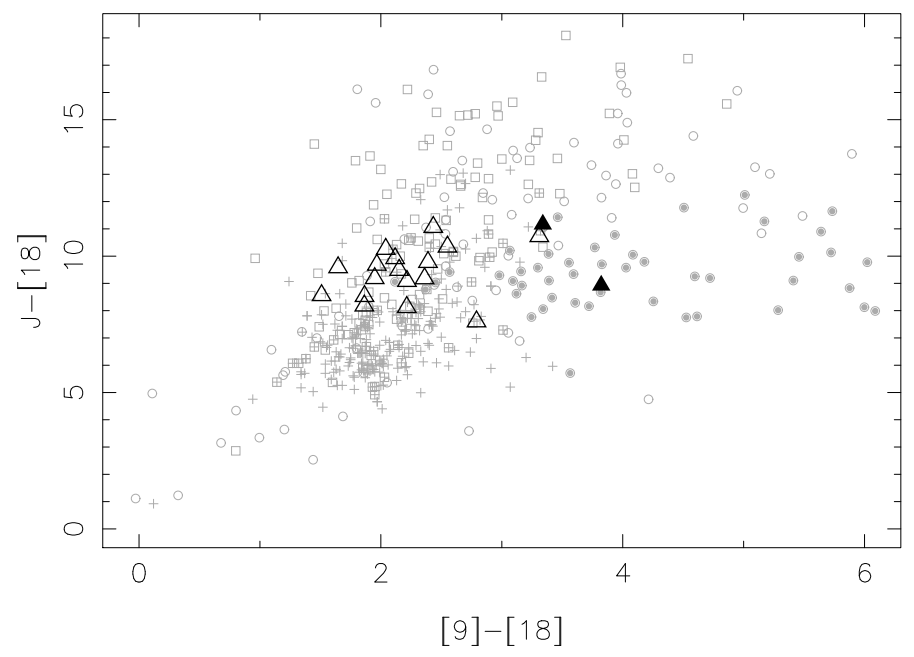

Fig. 7. Mid-IR color-color diagram based on AKARI fluxes. Triangles indicate our sample and gray circles represent the very likely post-AGBs from the Torun Catalog. Filled symbols are used for objects having double peaks in the SED, while open symbols represent single peaks. The distribution of young objects is shown by gray squares (YSOs detected by Spitzer) and gray crosses (HAeBe stars from $H B C$ ).

the AKARI data when compared to IRAS colors, the overlap of different categories also occurs in Fig. 7, distinguishing neither young objects nor evolved stars. Most of our sample is located in this overlap region, but three sources (PDS 204, 394, and 543) coincide with the region of post-AGBs, appearing more clearly separated, outside the main locus of the HAeBe stars, for example.

\subsubsection{Near-IR}

Near-IR photometry can be used to check whether the main source of emission is photospheric or nebular or if due to the presence of a circumstellar envelope. Figure 8 shows the $J-H$ vs. $H-K$ diagram studied by GL97 to compare AGB and post-AGB stars with different categories of objects, such as YSOs (region III), main sequence (region I/II), T Tauri and HAeBe stars (region III/IV), and planetary nebulae. GL97 verified that about $2 / 3$ of the planetary nebulae are found in the "nebulae box" (region V) defined by Whitelock (1985). The very likely post-AGB stars classified by Szczerba et al. (2007) are also plotted to illustrate the distribution of evolved objects with single or double peaks in their SED.

Bessell \& Brett (1988) studied the IR colors of long-period variables (LPV), carbon stars, and late-type supergiants. In Fig. 8 we plot the schematic areas presented by Bessell \& Brett (1988, Fig. A3), where carbon-rich stars are enclosed by dashed lines and oxygen-rich LPVs fall in the area defined by continuous line. The post-AGBs of our sample are located in region III from GL97, beyond the righthand-end schematic area from Bessell \& Brett. The only object coinciding with the region of carbon-rich stars is PDS551.

It can be noted in Fig. 8 that several of our stars are found in the same region as post-AGBs with single-peak SEDs, while our objects with double-peak SEDs appear in region I. As mentioned in Sect. 1.2, Siódmiak et al. (2008) verified a clear separation of nebulosity morphological classes SOLE and DUPLEX in near-IR diagrams. The $J-H$ vs. $H-K$ diagram in Fig. 8 shows the regions occupied by DUPLEX $(J-H>1)$ and SOLE (lower left side, $H-K<0.75$ ). It is interesting to note that,

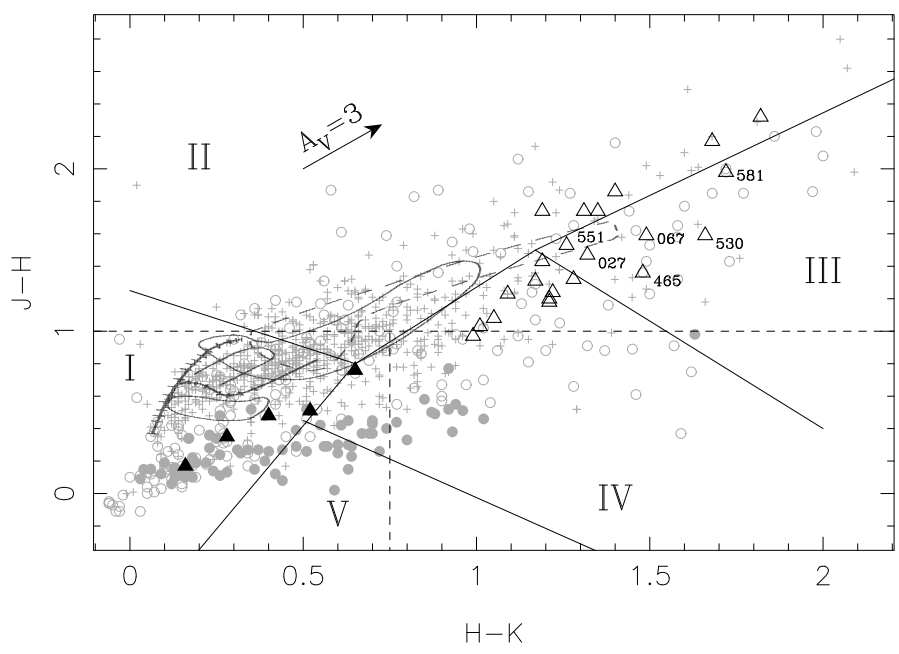

Fig. 8. Near-IR color-color diagram indicating the regions for different categories of objects defined by GL97 (separated by thin full lines), Siódmiak et al. (2008 - thin dashed lines), and Bessell \& Brett (1988 thick lines). Triangles are used to show our stars and gray circles represent very likely post-AGBs from the Torun Catalog. Double-peak SEDs are indicated by filled symbols (post-AGBs class IV). Gray crosses represent the HAeBe stars from $H B C$.

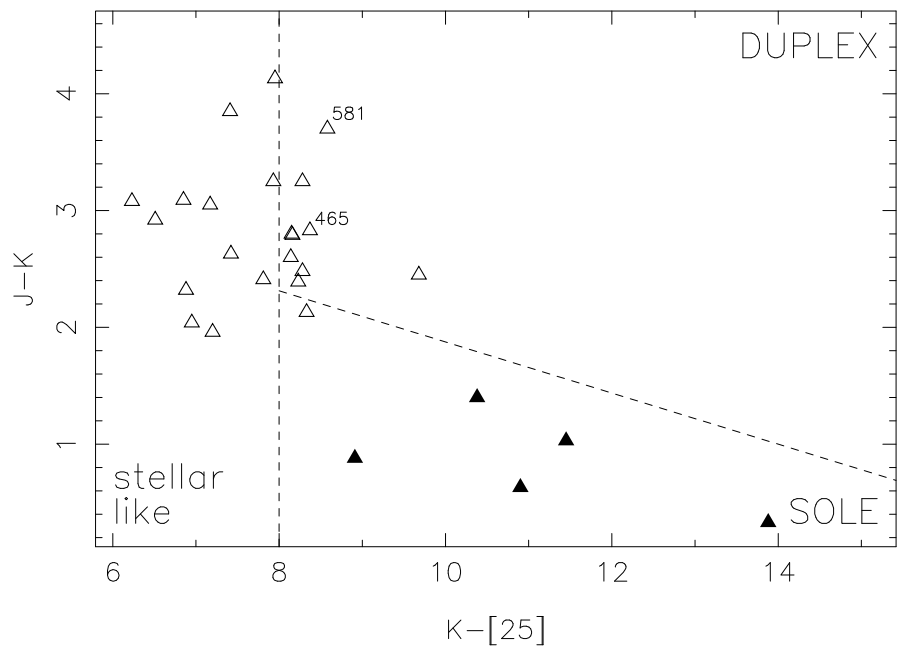

Fig. 9. Near- and mid-IR colors diagram indicating the regions defined by Siódmiak et al. (2008) (dashed lines). Filled triangles are used to show those of our stars with double peaked SEDs, while open triangles represent single peak SEDs.

in this diagram, the locus of the "stellar-like" objects dubbed by Siódmiak et al. (2008) (lower right side) coincides with the region IV, which is expected for TTs and HAeBe stars (GL97).

Comparisons of SED shapes are also interesting to discuss on the basis of near- to mid-IR excess, which is related to the morphology of a circumstellar structure (Ueta et al. 2000, 2007). Figure 9 presents the $J-K$ vs. $K-[25]$ diagram where stellar-like post-AGBs are in the left part $(K-[25]<8)$, while to the right are DUPLEXes (upper side) and SOLEs (lower part). Siódmiak et al. (2008) also correlate the SED shape with morphological groups: post-AGB class IV objects (double peak) are SOLE type, while classes I, II, or III (single peak) are DUPLEX. As can be seen in Fig. 9, the distribution of our double-peak sources coincides with the SOLEs region. We consider these sources, along with those located in the DUPLEX region, as possible post-AGB stars. 
In spite of the good correlations between the near-IR and SED shape, the discussion of elliptical or bipolar nebulosity types is only tentative since the morphology is unknown for most of our objects. One exception is PDS204, for which Perrin et al. (2009) used IR imagery and polarimetric data to confirm the presence of an edge-on disk surrounded by an extensive envelope pierced by bipolar outflow cavities. The results of the present work indicate that PDS204 has similar characteristics to post-AGBs, in particular its circumstellar morphology inferred from $J-K \times K-$ [25] colors, which is said to be DUPLEX (bipolar).

\section{Discussion and summary of results}

To analyze the similarities and differences between our sample of PDS sources and post-AGBs studied in other works, we established several criteria to diagnose whether the selected objects are pre-MS or not. The main result of this comparative study is the nomination of the likely post-AGB stars, indicated by notes in Table 2. For each given note, the adopted criteria and respective values that we consider typical characteristics of evolved objects are summarized as follows.

(a) The possible association with clouds was investigated by estimating the projected distance to the edge of the nearest cloud. According to the results presented in Sect. 3.1, note (a) indicates the objects having $d_{\text {edge }}>0.5^{\circ}$.

(b) The circumstellar extinction, defined by $f_{A v}$, was estimated by subtracting interstellar extinction, obtained from the Dobashi et al. $A_{\mathrm{V}}$ maps, of the total visual extinction, obtained from the $E(B-V)$ calculation. The fraction of source emission at $100 \mu \mathrm{m}$, defined by $f_{F 100}$, was also evaluated to verify possible interstellar cirrus contamination. Note (b) indicates the objects showing $f_{A \mathrm{v}}>0.5$ and $f_{F 100}>0.5$, as described in Sect. 3.3.

(c) Our sample was also analyzed according to the spectral features, in particular the equivalent width of $\mathrm{H}_{\alpha}$ line. In Fig. 4 the strength of $\mathrm{H}_{\alpha}$ is compared to the fractions of circumstellar emission $f_{\mathrm{Sc}}$ and $f_{A \mathrm{v}}$. Note (c) indicates all the objects showing $W_{\mathrm{H} \alpha}>50 \AA$ that have $f_{\mathrm{Sc}}>0.87$ and $f_{A \mathrm{v}}>0.5$.

(d) In Fig. 5 (optical color-color diagram), seven objects have $U-B>-1$ and $B-V<-0.2$, which we consider indicative of $U-B$ excess typical of post-AGBs. As discussed in Sect. 5.1, no difference in gravities could be verified in the case of high temperatures, but one object with $B-V>0.1$ seems to have a low gravity, a possible indicator of evolved status.

(e) As mentioned in Sect. 5.2.1, the IRAS color-color diagram (Fig. 6) shows eight of our objects with [12]- [25] $>1$, all of them with $F_{12}>2 \mathrm{Jy}$. This criterion has been suggested by van der Veen et al. (1989) to distinguish young stars, associated to clouds, from post-AGBs located in this part of the diagram that typically show $F_{12}>2 \mathrm{Jy}$.

(f, g) The distribution of our sample in diagrams of near-IR colors was checked according the suggestion by GL97 that establishes different regions of these diagrams for each evolutive post-AGB phase. The objects located in regions I and III of Fig. 8 are indicated by note (f). The diagram of nearand mid-IR colors shown in Fig. 9 indicates that our objects with double-peak SED are placed in the region of SOLE type post-AGBs and single-peak sources coincide with DUPLEX objects, as suggested by Siódmiak et al. (2008). According to the results discussed in Sect. 5.2.3, objects with $K-[25]>8$ have note $(\mathrm{g})$.

\section{Conclusions and perspectives for future works}

The whole set of adopted criteria suggests that $26 \%$ (7/27 sources) of the studied sample are very likely post-AGB stars that achieved five or more notes. Four objects with three or four notes are considered possible post-AGBs. As discussed below, PDS371 is also included in this list indicating that $18 \%$ $(5 / 27)$ of the sample possibly are post-AGBs. Among the other objects with fewer than three notes, $8 / 27$ sources $(30 \%$ of the sample) are unlikely post-AGB since they were confirmed in the literature as pre-MS stars. The nature of the remaining 7/27 objects is unclear. Individual comments are given in Appendix A to present recent results from literature related to the nature of our stars.

PDS465 and 581 are well known post-AGBs. The other very likely post-AGBs indicated by us are PDS 27, 174, 204, 216, and 353. Results from other works support these suggestions at least for two of them: PDS 27 (Suárez et al. 2006) and PDS 174 (Sunada et al. 2007).

The unlikely post-AGB objects: PDS 18, 37, 141, 168, 193, 198, 406, and 518 are confirmed pre-MS stars (see Appendix A). Other sources with less than three notes are PDS 67, 207, 257, $477,520,530$, and 551, which were not previously studied in the literature.

Other objects whose nature remains to be confirmed are PDS 290, 394, 431, 543, which we suggest are possible postAGB objects. As discussed in Sect. 4, PDS371 is included in this group since it shows spectral features similar to proto-PN. The AKARI colors, discussed in Sect. 5.2.2, were not used to nominate the post-AGBs, owing to the position of our sample, which is mainly concentrated in the region of overlap of young and evolved objects in Fig. 7. However, it is interesting to note that three sources appear out of this overlap region. Indeed, PDS 204 (very likely post-AGB), PDS 394, and 543 (possible postAGBs) have colors [9] - [18] > $3 \mathrm{mag}$, which coincide with the locus of most of the post-AGB class IV, confirming the classification that we suggest for these PDS sources.

We are aware that additional observations are needed to definitively classify the candidates, as mentioned by Szczerba et al. (2007) in their evolutive catalog of post-AGB objects. Several techniques and instruments operating in different spectral domains can be used to confirm the evolved nature of our candidates. Detailed spectroscopic analysis is required to better determine spectral type and luminosity class, which could be evaluated through the spectral lines sensitive to changes in gravity (Pereira \& Miranda 2007). Spectral synthesis, for example, would provide abundances of $\mathrm{C}$ and $\mathrm{O}$, whose ratio is used in the classification of post-AGB stars (van der Veen et al. 1989). Determing the abundances of $\mathrm{Ba}$ and $\mathrm{Sr}$ is also interesting, since the enrichment of elements produced by s-process is expected to occur during the post-AGB phase (Parthasarathy 2000). However, a detailed spectral analysis of absorption lines is constrained to objects with intermediary to low $T_{\text {eff }}$, which is not the case for our sources, which do not show these spectral features. It is better, in this case, to study the emission line profile of He I $\lambda 5876$ or the strength of [N II] $\lambda 5754$ relative to [O I] $\lambda$ 6302, which were used by Pereira et al. (2008), for example, to indicate the nature of a proto-PN candidate. Evidence of a carbon-rich chemistry can also be obtained through mid-infrared spectroscopy from 10 to $36 \mu \mathrm{m}$, as in Hrivnak et al. (2009).

Direct imagery remains the most interesting tool for revealing the morphology of the nebulae. Our sources show several indications of a considerable amount of circumstellar material that could be detected in high-resolution images. 
Ueta et al. (2007), for example, studied the post-AGB circumstellar structures via dust-scattered linearly polarized starlight by using imaging-polarimetric data obtained with NICMOS/HST. It would be very interesting to develop the SED fitting based on more reliable circumstellar parameters and a more realistic disk model than adopted in the present work. We intend to apply a radiative transfer model specifically developed to studying the physical conditions such as mass loss and envelope density of the post-AGB.

Our results indicate new likely and possible post-AGBs that open promising and exciting perspectives for continued studies of these targets.

Acknowledgements. R.G.V. and J.G.H. thank support from FAPESP (Proc. Nos. 2008/01533-4; 2005/00397-1). This research made use of the SIMBAD database, operated at the CDS, Strasbourg, France.

\section{Appendix A: Individual comments}

The literature has been searched for results confirming the nature of the studied objects, which are found for $15 / 27$ of the sample, all of them consistent with the results of our work. In this section we separate these references into three parts: (i) previously known young stars; (ii) very likely post-AGBs; (iii) possible post-AGB or pre-MS.

\section{A.1. Young stars}

PDS18 is included in the study of YSOs showing IR nebula, presented by Conneley et al. (2007). A reflection nebulae associated to PDS18 is cataloged by Magakian (2003). Conneley et al. (2008) also verified the presence of an arc-shaped nebula ( $\sim 8$ arcsec to the northeast) around this object.

PDS141 (DK Cha), one of the most studied object of our sample, is a Class I pre-MS star. Results based on Spitzer data have been presented by Porras et al. (2007), Alcalá et al. (2008), Ybarra \& Lada (2009), Evans et al. (2009), for example. The disk structure was studied by Liu et al. (2007). CO emission has been reported by Otrupcek et al. (2000).

Spitzer data are also provided for PDS168 (Luhman et al. 2006; Furlan et al. 2008), which is considered a Class I protostar (White \& Hillenbrand 2004; Terebey et al. 2009). The protoplanetary disk was studied from $\mathrm{H}_{2}$ emission (Bitner et al. 2008) and from millimeter data (Andrews \& Williams 2005).

$P D S 193$ is in the $3 \mu \mathrm{m}$ spectroscopic survey of HAeBes, presented by Acke \& van den Ancker (2006). PDS406 was included in the study of Class 0 protostars by Froebrich (2005) and appears in the reflection nebulae catalog of Magakian (2003).

Sunada et al. (2007) include PDS198 in their study of $\mathrm{H}_{2} \mathrm{O}$ maser, but no maser emission is found for this object, probably due to its intermediate-mass (F0 type star). Acke \& van den Ancker (2006) consider PDS198 as an HAeBe.

PDS518 is also a well-studied young B star (Acke et al. 2008), for which $3 \mu \mathrm{m}$ spectroscopy of protoplanetary disks is presented by Goto et al. (2009) and the circumstellar structure was also studied by Alonso-Albi et al. (2009). Conneley et al. (2008) included PDS518 in a study of multiplicity of embedded protostars.

\section{A.2. Post-AGB}

PDS27 appears in the list of Suárez et al. (2006) who presented a Spectroscopic Atlas of post-AGB stars. CO emission has been reported by Urquhart et al. (2007).
The emission of $\mathrm{H}_{2} \mathrm{O}$ maser (usually found in massive protostars) was not detected in the survey by Sunada et al. (2007) in the case of $P D S 174$, which is a result consistent with our suggestion of post-AGB candidate for this object.

As stated before, PDS465 and PDS581 are confirmed postAGB objects largely studied in the literature. The most recent results for PDS465 are presented by Lee et al. (2009), De Marco (2009), Luna et al. (2008), Raga et al. (2008), among others, and for PDS 581: Steffen et al. (2009), Montez et al. (2009), Dennis et al. (2008), Sanchez Contreras et al. (2008), among others. CO emission associated to PDS581 has been reported by Urquhart et al. (2008). Both objects are listed in the Torun catalog, classified by Szczerba et al. (2007) as IRAS selected source (PDS465) or reflection nebulosity (PDS581).

PDS 394 is studied as post-AGB candidate by Suárez et al. (2006) and Szczerba et al. (2007).

PDS204 (MWC778) is considered a peculiar object in the $\mathrm{H}_{2} \mathrm{O}$ maser study of post-AGB (Suárez et al. 2007, 2006). CO emission is reported by Kawamura et al. (1998) and Urquhart et al. (2007). Herbig \& Vacca (2008) suggest that MWC778 has the SED slope of a pre-MS embedded object, which has spectral type F or G, due to the metallic absorption lines. From IR imagery and polarimetric data, Perrin et al. (2009) confirm the presence of an edge-on disk surrounded by an extensive envelope pierced by bipolar outflow cavities. They argue that MWC778 has high bolometric luminosity inconsistent with $\mathrm{F}$ or $\mathrm{G}$ type star. The results of the present work indicate that PDS204 has similar characteristics to post-AGBs, in particular its circumstellar morphology inferred from $J-K \times K-$ [25] colors, which are found in DUPLEX (bipolar) post-AGBs.

$P D S 216$ has been included in the study of hydrocarbon molecules in Herbig Ae stars (Acke et al. 2010) aiming to verify the correlation of effective temperature with the $7.8 \mu \mathrm{m}$ feature, when comparing their sample with low- and high-mass stars. Considering that similar results are also found for S-type AGB stars (Smolders et al. 2010), Acke et al. (2010) conclude that the chemistry of hydrocarbon molecules in the circumstellar environment is mainly affected by stellar radiation field regardless of the evolutionary status.

\section{A.3. Possible post-AGB or pre-MS}

PDS37 is in the list of post-AGB stars studied by Szczerba et al. (2007), but it is considered YSO in the work of Mottran et al. (2007) and in the Spitzer mid-IR survey by Carpenter et al. (2008). CO emission has been reported by Urquhart et al. (2007).

PDS371 has been included in the photometry and spectroscopy for luminous stars catalog by Reed (2005). However, no additional information is available than those given by the PDS catalog (Vieira et al. 2003).

CO emission has been reported by Brand et al. (1987) for PDS290 and PDS353.

Pestalozzi et al. (2005) detected methanol maser emission in PDS431, which usually is considered an indicator of massive star formation and is observed in Class 0 protostars. $\mathrm{CO}$ emission has been reported by Urquhart et al. (2007).

\section{References}

Acke, B., \& Van den Ancker, M. E. 2006, A\&A, 457, 171

Acke, B., Verhoelst, T., Van den Ancker, M. E., et al. 2008, A\&A, 485, 209

Acke, B., Bouwman, J, Juhász, A., et al. 2010, ApJ, 718, 558

Adams, F. C., \& Shu, F. H. 1986, ApJ, 308, 836

Alcalá, J. M., Spezzi, L., Chapman, N., et al. 2008, ApJ, 676, 427 
Alecian, E., Catala, C., Wade, G. A., et al. 2008, MNRAS, 385, 391 Alonso-Albi, T., Fuente, A., Bachiller, R., et al. 2009, A\&A, 497, 117 Andrews, S. M., \& Williams, J. P. 2005, ApJ, 631, 1134

Antonopoulos, E., \& Pottasch, S. R. 1987, A\&A, 173, 108

Bessel, M. S., \& Brett, J. M. 1988, PASP, 100, 1134

Bessel, M. S., Castelli, F., \& Plez, B. 1998, A\&A, 333, 231

Beskrovnaya, N. G., Pogodin, M. A., Najdenov, I. D., \& Romanyuk, I. I. 1995, A\&A, 298, 585

Bitner, M. A., Richter, M. J., Lacy, J. H., et al. 2008, ApJ, 688, 1326 Bowers, P. F., \& Knapp, G. R. 1989, ApJ, 347, 325 Brand, J., Blitz, L., Wouterloot, J. G. A., \& Kerr, F. J. 1987, A\&A, 68, 1 Cardelli, J. A., Cleyton, G. C., \& Mathis, J. S. 1989, ApJ, 345, 245 Carpenter, J. M., Bouwman, J., Silverstone, M. D., et al. 2008, ApJS, 179, 423 Catala, C., Donati, J. F., Böhm, T., et al. 1999, A\&A, 345, 884 Connelley, M. S., Reipurth, B., \& Tokunaga, A. T. 2007, AJ, 133, 1528 Connelley, M. S., Reipurth, B., \& Tokunaga, A. T. 2008, AJ, 135, 2496 de Jager, C., \& Nieuwenhuijzen, H. 1987, A\&A, 177, 217

De Marco, O. 2009, PASP, 121, 316

Dennis, T. J., Cunninghan, A. J., Frank, A., et al. 2008, ApJ, 679, 1327

Dobashi, K., Uehara, H., Kandori, R., et al. 2005, PASJ, 57, SP1

Epchtein, N., Le Bertre, T., \& Lépine, J. R. D. 1990, A\&A, 227, 82

Evans, N. J., Dunham, M. M., Jorgensen J. K., et al. 2009, ApJS, 181, 321

Feitzinger, J. V., \& Stüwe, J. A. 1984, A\&AS, 58, 365

Feast, M. W., \& Whitelock, P. A. 1987, in Late Stages of Stellar Evolution, ed.

S. Kwok, \& S. R. Pottasch (Dordrecht: Reidel), 33

Froebrich, D. 2005, ApJS, 156, 169

Furlan, E., McClure, M., Calvet, N., et al. 2008, ApJS, 176, 184

García-Lario, P., Manchado, P., Pych, W., \& Pottasch, S. R. 1997, A\&AS, 126, 479

Gregorio-Hetem, J., Lépine, J. R. D., Quast, G. R., Torres, C. A. O., \& de la Reza, R. 1992, AJ, 103, 549

Gregorio-Hetem, J., \& Hetem, A. Jr. 2002, MNRAS, 336, 197 Goto, M., Henning, Th., Kouchi, A., et al. 2009, ApJ, 693, 610

Gutermuth, R. A., Megeath, S. T., Myers, P. C., et al. 2009, ApJS, 184, 18 G Harris, S., Clegg, P., \& Hughes, J. 1988, MNRAS, 235, 441

Herbig, G. H., \& Bell, K. R. 1988, Third Catalog of Emission-Line Stars of the Orion Population, Lick Observatory Bulletin, 1111

Herbig, G., \& Vacca, W. D. 2008, AJ, 136, 1995

Hillenbrand, L. A., Strom, S. E., Vrba, F. J., \& Keene, J. 1992, ApJ, 397, 613

Hrivnak, B. J., Volk, K., \& Kwok, S. 2009, ApJ, 694, 1147

Ishihara, D., Onaka, T., Kataza, H., et al. 2010, A\&A, 514, A1

Ita, Y., Matsuura, M., Ishihara, D., et al. 2010, A\&A, 514, A2

Jablonski, F., Baptista, R., Barroso, F., Jr., et al. 1994, PASP, 106, 1172

Kawamura, A., Onishi, T., Yonekura, Y., et al. 1998, ApJS, 117, 387

Lee, C.-F., Hsu, M.-C., \& Sahai, R. 2009, ApJ, 696, 1630

Liu, W. M., Hinz, P. M., Meyer, M. R., et al. 2007, ApJ, 658, 1164

Luhman, K. L., Whitney, B. A., Meade, M.R., et al. 2006, ApJ, 647, 1180

Luna, R., Cox, N. L. J., Satorre, M. A., et al. 2008, A\&A, 480, 133

Lynds, B. T. 1962, ApJS, 7, 1

Magakian, T. Y. 2003, A\&A, 399, 141

Malfait, K., Bogaert, E., \& Waelkens, C. 1998, A\&A, 331, 211

Meeus, G., Waters, L. B. F. M., Bouwman, J., et al. 2001, A\&A, 365, 476

Montez, R., Kastner, J. H., Balick, B., \& Frank, A. 2009, ApJ, 694, 1481

Mottram, J. C., Hoare, M. G., Lumsden, S. L., et al. 2007, A\&A, 476, 1019

Otrupcek, R. E., Hartley, M., \& Wang, J.-S. 2000, PASA, 17, 92
Parthasarathy, M. 2000, IAUS, 177, 225

Pereira, C. B., \& Miranda, L. F. 2005, A\&A, 433, 579

Pereira, C. B., \& Miranda, L. F. 2007, A\&A, 462, 231

Pereira, C. B., Marcolino, W. L. F., Machado, M., \& de Araújo, F. X. 2008, A\&A, 477, 877

Perrin, M. D., Vacca, W. D., \& Graham, J. R. 2009, AJ, 137, 4468

Pestalozzi, M. R., Minier, V., \& Booth, R. S. 2005, A\&A, 432, 737

Porras, A., Jorgensen, J. K., Allen, L. E., et al. 2007, ApJ, 656, 493

Raga, A. C., Riera, A., Mellema G., et al. 2008, A\&A, 489, 1141

Riera, A., García-Lario, P., Manchado, A., Potash, S. R., \& Raga, A. C. 1995, A\&A, 302, 137

Reed, B. C. 2005, AJ, 130, 1652

Rodrigues, C. V., Jablonski, F. J., Gregorio-Hetem, J., Hickel, G. R., \& Sartori, M. J. 2003, ApJ, 587, 312

Rodrigues, C. V., Sartori, M. J., Gregorio-Hetem, J, \& Magalhães, A. M. 2009, ApJ, 698, 2031

Rowan-Robinson, M. 1986, MNRAS, 219, 737

Sanchez Contreras, C., Sahai, R., Gil de Paz, A., \& Goodrich, R. 2008, ApJS, 179,166

Sartori, M. J., Gregorio-Hetem, J., Rodrigues, C. V., Hetem, A., \& Batalha, C. 2010, AJ, 139, 27

Savage, B. D., \& Mathis, J. S. 1979, ARA\&A, 17, 73

Schultz, G. V., \& Wiemer, W. 1975, A\&A, 43, 133

Siódmiak, N., Meixner, M., Ueta, T., et al. 2008, ApJ, 677, 382

Sivagnaman, P. 1989, Ph.D. Thesis, University of Paris VII, France

Smolders, K., Acke, B., Verhoelst, T., et al. 2010, A\&A, 514, L1

Steffen, M., Szczerba, R., \& Schönberner, D. 1998, A\&A, 337, 149

Steffen, W., Garcia-Segura, G., \& Koning, N. 2009, ApJ, 691, 696

Straizys, V., \& Kuriliene, G. 1981, Ap\&SS, 80, 353

Strom, S. E., Strom, K. M., \& Grasdalen, G. L. 1975, ARA\&A, 13, 187

Suárez, O., García-Lario, P., Manchado, P., Manteiga, U., \& Pottasch, S. R. 2006, A\&A, 458, 173

Suárez, O., Gómez, J. F., \& Morata, O. 2007, A\&A, 467, 1085

Sunada, K., Nakazato, T., Ikeda, N., et al. 2007, PASJ, 59, 1185

Szczerba, R., Siódmiak, N., Stasińska, G., \& Borkowski, J. 2007, A\&A, 469, 799

Terebey, S., Fich, M., \& Noriega-Crespo, A. 2009, ApJ, 696, 1918

Torres, C. A. O., Quast, G. R., de la Reza, R., Gregorio-Hetem, J., \& Lépine, J. R. D. 1995, AJ, 109, 2146

Torres, C. A. O. 1999, Publication of CNPq/Observatório Nacional (Brazil), 10, 1

Ueta, T., Meixner, M., \& Bobrowsky, M. 2000, ApJ, 528, 861

Ueta, T., Murakawa, K., \& Meixner, M. 2007, AJ, 133, 1345

Urquhart, J. S., Busfield, A. L., Hoare, M. G., et al. 2007, A\&A, 474, 891

Urquhart, J. S., Busfield, A. L., Hoare, M. G., et al. 2008, A\&A, 487, 253

Van den Ancker, M. 1999, Ph.D. Thesis, University of Amsterdam

Van der Veen, W. E. C. J., \& Habing, H. J. 1988, A\&A, 194, 125

Van der Veen, W. E. C. J., Habing, H. J., \& Geballe, T. R. 1989, A\&A, 226, 108

Vieira, S. L. A., Corradi, W. J. B., Alencar, S. H. P., et al. 2003, AJ, 126, 2971

Winckel, H. V. 2003, ARA\&A, 41, 391

White, R. J., \& Hillenbrand, L. A. 2004, ApJ, 616, 998

Whitelock, P. A. 1985, MNRAS, 237, 479

Whitelock, P. A., Feast, M., \& Catchpole, R. 1991, MNRAS, 248, 276

Ybarra, J. E., \& Lada, E. A. 2009, ApJ, 695, L120 